\title{
Multicolinearidade em modelos de regressão logística
}

\author{
Karina Gernhardt Nakamura
}

\section{DISSERTAÇÃO APRESENTADA}

$\mathrm{AO}$

Instituto DE MATEMÁtica e EstatísticA

DA

Universidade DE SÃo PAUlo

PARA

OBTENÇÃO DO TÍTULO

$\mathrm{DE}$

Mestre em CiÊnCIAS

Programa: Estatística

Orientador: Profa. Dra. Silvia Nagib Elian

São Paulo, Maio de 2013 


\title{
Multicolinearidade em modelos de regressão logística
}

\author{
Esta dissertação contém as correções e alterações \\ sugeridas pela Comissão Julgadora durante a defesa \\ realizada por Karina Gernhardt Nakamura em 21/03/2013. \\ O original encontra-se disponível no Instituto de \\ Matemática e Estatística da Universidade de São Paulo.
}

Comissão Julgadora:

- Profa. Dra. Silvia Nagib Elian (orientadora) - IME-USP

- Profa. Dra. Carmen Diva Saldiva de André - IME-USP

- Prof. Dr. João Ricardo Sato - UFABC 


\section{Agradecimentos}

Conforme algumas pessoas já me disseram, quanto mais longa é a sua vida acadêmica mais solitário você será. Eu tenho que discordar disso. Ao longo desses três anos de dedicação e comprometimento com o Mestrado, eu posso dizer que conheci, reconheci e fortaleci ainda mais algumas amizades que levarei para sempre comigo. Portanto, eu gostaria de deixar aqui o meu muito obrigada para aquelas pessoas que participaram ativa ou passivamente dessa jornada:

Aos meus pais, Elaine e Armando, pelo amor incondicional, pela compreensão nos momentos difíceis e pelo orgulho que sempre me motiva a continuar indo além. A vocês, a minha eterna gratidão.

À minha irmã, Camila, e, porque não, à nossa "filha" Mel, por todo o amor/lambidas, pelas inúmeras palavras de carinho/mordidas e por sempre estarem ao meu lado. Vocês são imprescindíveis na minha vida.

Ao meu namorado, Luiz, que sempre apoiou as minhas decisões e que teve muita pacência nos momentos de inquietação e, principalmente, de cansaço. Com você descobri o que é o amor de verdade. Obrigada por fazer parte de mim.

À minha amiga-orientadora, Silvia, muito obrigada por me guiar e me motivar, pelas suas recomendações sempre muito pertinentes, pela liberdade me dada e pelo sucesso alcançado. Sou imensamente agradecida por tanta generosidade, carinho e aprendizado.

Aos meus queridos e para sempre chefes, Karin e Tiago, que me ensinaram que o Mestrado exige dedicação e que sempre foram muito compreensivos com relação aos meus horários e necessidades. Vocês são a minha inspiração como pessoa dentro e fora do trabalho.

Às minhas amigas Carol, Laís e Mari que muitas vezes tiveram que entender que a distância física não significa esquecimento. Obrigada por me apoiarem sempre.

Aos meus queridos amigos que me ajudaram muito no Mestrado, Gleyce e Bruno, e a todos aqueles que me motivaram ao longo desses três anos, Akina, Guilherme, Renata, Vanessa, Dudu, Rodrigo e Gabriel. Obrigada pelas suas contribuições, pelo ombro amigo e pelos momentos de descontração que foram essenciais para que eu conseguisse finalizar mais essa etapa da minha vida.

A todos os meus familiares, pelas palavras de apoio e de motivação sempre que precisei.

Todos vocês são co-autores deste trabalho. 


\section{Resumo}

\section{Multicolinearidade em modelos de regressão logística}

Neste trabalho estudamos os efeitos da multicolinearidade em modelos de regressão logística e apresentamos estimadores viesados para que tais efeitos fossem minimizados. Primeiramente, o modelo de regressão logística e o processo para a estimação dos parâmetros foram apresentados. Foram feitos, também, alguns testes para avaliar a significância dos mesmos, bem como técnicas para analisar a qualidade do ajuste do modelo. Em seguida, os efeitos da multicolinearidade na estimação dos parâmetros e na sua inferência foram avaliados, bem como técnicas para o seu diagnóstico. Para amenizar o efeito deste problema, apresentamos dois estimadores alternativos ao de máxima verossimilhança: estimador em cristas e estimador em componentes principais. Comparamos, então, o desempenho dos três estimadores na forma de um estudo de simulação e de uma aplicação em um conjunto de dados reais. O principal resultado obtido foi que, na presença de multicolinearidade, os estimadores alternativos conseguiram um melhor ajuste em comparação ao de máxima verossimilhança, além de minimizar os seus efeitos.

Palavras-chave: regressão logística, multicolinearidade, estimador em cristas, estimador em componentes principais. 


\section{Abstract}

\section{Multicollinearity in logistic regression models}

This work proposes the use of some biased estimators to investigate whether is possible minimize the multicollinearity effects in logistic regression models. Initially, the latter model was presented, as well as its fitting process (therefore obtaining the maximum likelihood estimator), some tests to evaluate the significance of the parameters and techniques to analyze goodness of fit were also considered. Furthermore, the effects of multicollinearity in the fitting process and in the parameters inference were discussed, as well as techniques to identify the presence of multicollinearity. In order to diminish the effect of this problem, two alternative estimators were presented: ridge estimator and principal component estimator. Therefore, these three estimators performances were compared using a simulation study and applied in a real data set. The manly conclusion was that, in the presence of multicollinearity, the alternative estimators performed better than the maximum likelihood estimator, besides reducing its effects.

Keywords: logistic regression, multicollinearity, ridge estimator, principal component estimator. 


\section{Sumário}

$\begin{array}{ll}\text { Lista de Figuras } & \text { ix }\end{array}$

Lista de Tabelas $\quad$ xi

1 Introdução $\quad 1$

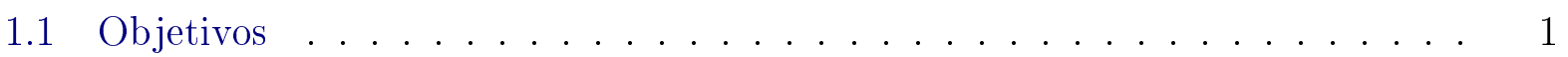

1.2 Organização do trabalho . . . . . . . . . . . . . . . . 2

2 Modelo de Regressão Logística $\quad 3$

2.1 Modelo de regressão logística . . . . . . . . . . . . . . . . . 3

2.2 Estimação dos parâmetros . . . . . . . . . . . . . . . . 7

2.3 Teste de significância dos parâmetros . . . . . . . . . . . . . . 9 9

2.4 Avaliação do ajuste do modelo . . . . . . . . . . . . . . . . . 10

2.5 Considerações finais do capítulo . . . . . . . . . . . . . . . . . . . 14

3 Efeitos e Diagnóstico da Multicolinearidade nos Modelos de Regressão $\begin{array}{ll}\text { Logística } & 15\end{array}$

3.1 Efeitos da multicolinearidade . . . . . . . . . . . . . 15

3.1.1 Decomposição em valores singulares da matriz de planejamento . . . 16

3.1.2 Efeitos da multicolinearidade na estimação dos parâmetros . . . . . . 18

3.1.3 Relação entre a multicolinearidade em modelos de regressão linear e logística . . . . . . . . . . . . . . . . 19

3.1.4 Efeitos da multicolinearidade na inferência sobre os parâmetros . . . 21

3.2 Diagnóstico da multicolinearidade . . . . . . . . . . . . . . . . . . . 22

3.2.1 Coeficiente de determinação . . . . . . . . . . . . . . . 23

3.2 .2 Índice e número de condição . . . . . . . . . . . . . 25

3.2 .3 Decomposição da variância . . . . . . . . . . . . . . . 26

3.3 Considerações finais do capítulo . . . . . . . . . . . . . . . . 27

4 Estimadores Alternativos para os Parâmetros do Modelo de Regressão $\begin{array}{ll}\text { Logística } & 29\end{array}$

4.1 Regressão em Cristas . . . . . . . . . . . . . . . . . . . . . . 29

4.1.1 Regressão Linear em Cristas . . . . . . . . . . . . . . . . . 29 
4.1.2 Regressão Logística em Cristas . . . . . . . . . . . . . . . . 33

4.1.3 Escolha do parâmetro em cristas . . . . . . . . . . . . . 34

4.2 Regressão em Componentes Principais . . . . . . . . . . . . . . . . 36

4.2.1 Estimador em Componentes Principais iterativo . . . . . . . . . . . 36

4.2.2 Estimador em Componentes Principais one-step . . . . . . . . . . . 38

4.2.3 Exclusão dos componentes principais . . . . . . . . . . . . . . 40

4.3 Considerações finais do capítulo . . . . . . . . . . . . . . . 40

5 Estudo de Simulação $\quad 43$

5.1 Descrição dos cenários e modelo . . . . . . . . . . . . . . . . . 43

5.2 Delineamento do estudo de simulação . . . . . . . . . . . . . . . . . . 44

5.3 Resultados . . . . . . . . . . . . . . . . . . . . 47

5.4 Considerações finais do capítulo . . . . . . . . . . . . . . 55

6 Aplicação em um Conjunto de Dados Reais $\quad 57$

6.1 Descrição do estudo . . . . . . . . . . . . . . . . . . . . . . 57

6.2 Identificação da multicolinearidade . . . . . . . . . . . . . . . 60

6.3 Aplicação dos estimadores viesados . . . . . . . . . . . . . . 65

6.3.1 Aplicação da Regressão em Cristas . . . . . . . . . . . . . . . . 65

6.3.2 Aplicação da Regressão em Componentes Principais . . . . . . . . . . 69

6.4 Considerações finais do capítulo . . . . . . . . . . . . . . . . 70

7 Considerações finais e sugestões para pesquisas futuras $\quad 73$

A Stepwise na Regressão Logística $\quad 75$

B Código em R do Estudo de Simulação $\quad 79$

C Código em R da Análise do Conjunto de Dados Reais $\quad 91$

D Tabelas dos Resultados da Aplicação 103

$\begin{array}{ll}\text { Referências Bibliográficas } & 121\end{array}$

$\begin{array}{ll}\text { Índice Remissivo } & 124\end{array}$ 


\section{Lista de Figuras}

2.1 Matriz de classificação. . . . . . . . . . . . . . . . . . . . . . . . . 13

2.2 Exemplo da curva ROC . . . . . . . . . . . . . . . . . . . . . 14

3.1 Interpretação geométrica da DVS no caso de duas covariáveis. . . . . . . 17

4.1 Comparação entre a soma das variâncias, o quadrado do viés e os erros quadráticos médios dos estimadores dos parâmetros em cristas e de mínimos quadrados em modelos de regressão linear. Fonte: Hoerl e Kennard (1970). . 31

4.2 Exemplo do gráfico do traço de Ridge. . . . . . . . . . . . . . . 35

6.1 Traço de Ridge com as estimativas dos parâmetros do modelo referente aos dados de aplicação. . . . . . . . . . . . . . . . . . . 66 


\section{Lista de Tabelas}

2.1 Distribuição de probabilidades de Y. . . . . . . . . . . . . 4

2.2 Diferenças entre o modelo de regressão linear e o modelo de regressão logística. 7

2.3 Classificação do poder de discriminação do modelo segundo a área abaixo da curva ROC. ................................ 14

3.1 DVS da matriz de planejamento com duas covariáveis. . . . . . . . . . . 16

3.2 Projeção das observações nos eixos alternativos. . . . . . . . . . . . . 17

3.3 Classificação da multicolinearidade. . . . . . . . . . . . . . . 25

3.4 Matriz com a Proporção da Decomposição da Variância. . . . . . . . . . . . 27

5.1 Média e erro padrão do índice de condição e do valor máximo do coeficiente de determinação de cada cenário. . . . . . . . . . . . . . 48

5.2 Média e erro padrão das estimativas do erro quadrático médio do estimador em cada modelo e cenário. . . . . . . . . . . . . . . 50

5.3 Quantidade e percentual de vezes em que cada estimador apresentou o menor erro quadrático médio. . . . . . . . . . . . . . . . 52

5.4 Quantidade e percentual de vezes em que cada parâmetro em cristas gerou estimativas com o menor erro quadrático. . . . . . . . . . . . 54

6.1 Estimativas dos parâmetros e dos erros padrão dos estimadores dos parâmetros do modelo de regressão logística com todas as covariáveis significantes univariadamente a um nível de 0,0001 . . . . . . . . . . . . 60

6.2 Coeficiente de determinação da regressão linear das covariáveis em relação às

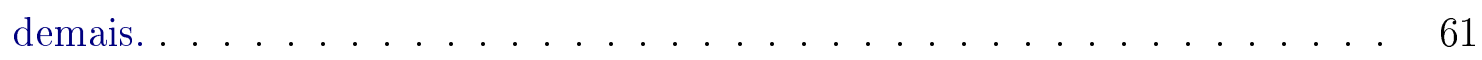

6.3 Decomposição em valores singulares da matriz de planejamento dos dados da aplicação. . . . . . . . . . . . . . . . . . 62

6.4 Índices de condição da matriz de planejamento dos dados da aplicação. . . . 63

6.5 Números de condição da matriz de planejamento dos dados da aplicação. . 63

6.6 Decomposição da variância dos estimadores de máxima verossimilhança. . . . 64

6.7 Valores para o parâmetro em cristas. . . . . . . . . . . . . 66 
6.8 Estimativas dos parâmetros e dos erros padrão dos estimadores dos parâmetros dos modelos de Regressão em Cristas, segundo o valor do parâmetro em cristas. . . . . . . . . . . . . . . . . 6 67

6.9 Tabelas para cálculo da estatística do Teste de Hosmer-Lemeshow para a Regressão em Cristas, segundo o valor do parâmetro em cristas. . . . . . . . 68

6.10 Valores das estatísticas e dos p-valores do Teste de Hosmer-Lemeshow e a variação da estatística dos modelos de Regressão em Cristas em relação ao de máxima verossimilhança, segundo o valor do parâmetro em cristas. . . . . . .

6.11 Estimativas dos parâmetros e dos erros padrão dos estimadores dos parâmetros do modelo de Regressão em Componentes Principais. . . . . . . . . . . . 69

6.12 Tabelas para cálculo do Teste de Hosmer-Lemeshow para a Regressão em Componentes Principais.

6.13 Valor da estatística e do p-valor do Teste de Hosmer-Lemeshow e a variação da estatística do modelo de Regressão em Componentes Principais em relação ao ajuste de máxima verossimilhança. . . . . . . . . . . . . . .

D.1 Descrição das variáveis da base de aplicação. . . . . . . . . . . . . . . . 103

D.2 Continuação da descrição das variáveis da base de aplicação. . . . . . . . . . . 104

D.3 Análise exploratória de HA. . . . . . . . . . . . . . . . . 104

D.4 Análise exploratória de DIAB . . . . . . . . . . . . . . 104

D.5 Análise exploratória de TABAG. . . . . . . . . . . . . . . 105

D.6 Análise exploratória de ANGEST. . . . . . . . . . . . . . . . . . . 105

D.7 Análise exploratória de ANGINS. . . . . . . . . . . . . 105

D.8 Análise exploratória de ICC . . . . . . . . . . . . . . 105

D.9 Análise exploratória de ARRIT. . . . . . . . . . . . . . . 105

D.10 Análise exploratória de ARTER . . . . . . . . . . . . . 106

D.11 Análise exploratória de CD . . . . . . . . . . . . . . 106

D.12 Análise exploratória de DA. . . . . . . . . . . . . 106

D.13 Análise exploratória de CX. . . . . . . . . . . . . . 107

D.14 Análise exploratória de GENERO . . . . . . . . . . . . . . . . 107

D.15 Análise exploratória de OBESO . . . . . . . . . . . . . . . 107

D.16 Análise exploratória de AH2 . . . . . . . . . . . . . . . . 107

D.17 Análise exploratória de INFARTO. . . . . . . . . . . . . 107

D.18 Análise exploratória de COLM, IDADFEM, IDADMAS, COLS, TRIGM, TRIGS, GLICM e GLICS. . . . . . . . . . . . . . . 108

D.19 Estimativas dos coeficientes e respectivos erros padrão entre parênteses dos modelos de regressão logística univariados de HA, DIAB, TABAG, ANGEST e ANGINS. . . . . . . . . . . . . . . . . . 109

D.20 Estimativas dos coeficientes e respectivos erros padrão entre parênteses dos modelos de regressão logística univariados de ICC, ARRIT, ARTER, CD e DA.109 
D.21 Estimativas dos coeficientes e respectivos erros padrão entre parênteses dos modelos de regressão logística univariados de CX, GENERO, OBESO, AH2 e INFARTO . . . . . . . . . . . . . . . . . . . . 110

D.22 Estimativas dos coeficientes e respectivos erros padrão entre parênteses dos modelos de regressão logística univariados de COLM, IDADFEM, IDADMAS e COLS . . . . . . . . . . . . . . . . . 110

D.23 Estimativas dos coeficientes e respectivos erros padrão entre parênteses dos modelos de regressão logística univariados de TRIGM, TRIGS, GLICM e

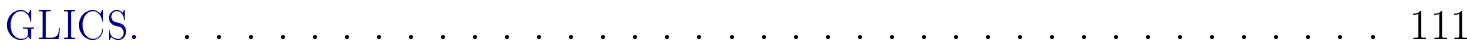

D.24 Modelos de regressão linear com variáveis resposta dadas por DIAB, TABAG, ANGEST e ANGINS. . . . . . . . . . . . . . . . . . . . . 111

D.25 Modelos de regressão linear com variáveis resposta dadas por ARRIT, GENERO, INFARTO e IDADFEM. . . . . . . . . . . . . . . . 112

D.26 Modelos de regressão linear com variáveis resposta dadas por IDADMAS, COLM, TRIGM e GLICM. . . . . . . . . . . . . . . . 113

D.27 Matriz de covariância estimada dos estimadores dos parâmetros do Modelo de Regressão Logística através de Máxima Verossimilhança. . . . . . . . . . . . 114

D.28 Matriz de covariância estimada dos estimadores dos parâmetros do Modelo de Regressão em Cristas com o primeiro parâmetro em cristas. . . . . . . . . 115

D.29 Matriz de covariância estimada dos estimadores dos parâmetros do Modelo de Regressão em Cristas com o segundo parâmetro em cristas. . . . . . . . 116

D.30 Matriz de covariância estimada dos estimadores dos dos parâmetros do Modelo de Regressão em Cristas com o terceiro parâmetro em cristas. . . . . . . . . 117

D.31 Matriz de covariância estimada dos estimadores dos parâmetros do Modelo de Regressão em Cristas com o quarto parâmetro em cristas. . . . . . . . . . 118

D.32 Matriz de covariância estimada dos estimadores dos parâmetros do Modelo de Regressão em Cristas com o quinto parâmetro em cristas. . . . . . . . . . 119

D.33 Matriz de covariância estimada dos estimadores dos parâmetros do Modelo de Regressão em Componentes Principais. . . . . . . . . . . . . . . . . 120 


\section{Capítulo 1}

\section{Introdução}

Apesar de ser grande o interesse por modelos de regressão logística (Hosmer e Lemeshow (2000), Agresti (1996), Hilbe (2009), Paula (1999)), pouco se comenta sobre o problema de multicolinearidade. A multicolinearidade ocorre quando existe uma relação exata ou aproximada entre as colunas da matriz de planejamento do modelo, fazendo com que seja introduzida uma singularidade aproximada nessa matriz e, consequentemente, produzindo instabilidade nas estimativas dos parâmetros obtidas através do método de máxima verossimilhança, nos seus respectivos erros padrão e nos resultados de alguns testes estatísticos. Como consequência desse fato, as conclusões e a infêrencia sobre os parâmetros baseada neste modelo poderão ficar seriamente comprometidas. Seu correto diagnóstico e técnicas para amenizar e até eliminar o problema e as suas consequências em modelos de regressão logística são raras na literatura.

Na presente dissertação serão analisados os efeitos da multicolinearidade na inferência e serão apresentados procedimentos para diagnosticar e quantificar esse problema, bem como métodos de estimação alternativos adequados na presença de multicolinearidade nos modelos de regressão logística.

\subsection{Objetivos}

Este estudo pretende contribuir para a difusão e maior esclarecimento do problema da multicolinearidade em modelos de regressão logística, além de estimular o uso das técnicas para o seu diagnóstico e prevenção. O objetivo é ilustrar, através de um estudo de simulação e de uma aplicação em um conjunto real de dados, quais as suas consequências e quais as condições necessárias para essas técnicas apresentarem um melhor desempenho em relação à técnica utilizada usualmente na estimação dos parâmetros do modelo de regressão logística. 


\subsection{Organização do trabalho}

O presente trabalho é dividido em sete capítulos, descritos resumidamente a seguir. No Capítulo 2, apresentamos o Modelo de Regressão Logística e todos os passos necessários para se obter o melhor ajuste. Em seguida, no Capítulo 3, serão avaliados os efeitos da multicolinearidade e descritas algumas formas de identificar e quantificar o nível de multicolinearidade entre as colunas da matriz de planejamento do modelo. Já no Capítulo 4, dois dos estimadores viesados mais utilizados para combater esse problema em modelos de regressão, estimador em cristas e estimador em componentes principais, serão expostos e as suas vantagens e desvantagens serão discutidas. No Capítulo 5 um estudo de simulação será desenvolvido com o objetivo de identificar em quais condições cada estimador consegue amenizar o problema da multicolinearidade e no Capítulo 6 as técnicas discutidas previamente serão aplicadas em um conjunto de dados reais. Por fim, no Capítulo 7 discutiremos algumas conclusões obtidas neste trabalho e apresentaremos sugestões para trabalhos futuros. 


\section{Capítulo 2}

\section{Modelo de Regressão Logística}

Regressão logística é uma das técnicas mais utilizadas na análise de uma variável resposta binária. O seu principal objetivo é descrever a relação entre a variável resposta (também conhecida como variável dependente) e as covariáveis (também conhecidas como variáveis explicativas) e é amplamente utilizada em diversas áreas. Na medicina, por exemplo, dois conceitos muito utilizados provenientes da regressão logística são o de razão de chances e o de risco do evento. Na área Financeira, modelos como behaviour scoring e credit scoring são largamente utilizados com o propósito de avaliar o risco dos atuais clientes honrarem o empréstimo concedido pelo banco e dos futuros clientes adquirirem produtos ou serviços, respectivamente. Essa técnica também é a base da Análise do Controle de Qualidade Seis Sigma e desempenha um papel muito importante na área de data mining.

Neste capítulo, vamos apresentar o modelo de regressão logística, suas suposições, o procedimento de estimação dos parâmetros e algumas medidas para avaliar a qualidade de ajuste do modelo.

\subsection{Modelo de regressão logística}

Quando uma variável resposta binária é função de mais do que uma variável explicativa, dizemos que este é um modelo de regressão logística múltipla, ou, simplesmente, um modelo de regressão logística. Neste modelo, cada elemento amostral tem as suas características padronizadas armazenadas em um vetor $\mathbf{x}_{i}$. Já a variável resposta binária associada ao iésimo indivíduo, que assume valores 0 e 1, será denotada por $Y_{i}$ e é definida como:

$$
Y_{i}= \begin{cases}1, & \text { se o i-ésimo elemento amostral possui a característica de interesse } \\ 0, & \text { caso contrário. }\end{cases}
$$

Da forma como foi definida, podemos notar que a variável resposta $Y_{i}$ tem distribuição Bernoulli com probabilidade de sucesso igual a $\pi\left(\mathbf{x}_{i}\right)$, em que $\pi\left(\mathbf{x}_{i}\right)=P\left(Y_{i}=1 \mid \mathbf{x}_{i}\right)$ é a probabilidade desconhecida de existência da característica de interesse, associada ao valor 
$\mathbf{x}_{i}$ das covariáveis padronizadas. A padronização utilizada será descrita adiante.

Sejam os pares $\left(Y_{1}, \mathbf{x}_{1}\right),\left(Y_{2}, \mathbf{x}_{2}\right), \ldots,\left(Y_{n}, \mathbf{x}_{n}\right)$, em que $\left(Y_{1}, Y_{2}, \ldots, Y_{n}\right)$ são as variáveis aleatórias independentes tais que $Y_{i}$ tem distribuição Bernoulli com probabilidade de sucesso igual a $\pi\left(\mathbf{x}_{i}\right)$ e $\mathbf{x}_{i}=\left(1, x_{i 1}, \ldots, x_{i p-1}\right)^{\prime}$ é um vetor $p \mathrm{x} 1$ contendo o valor 1 na primeira posição e os valores padronizados das covariáveis do i-ésimo indivíduo nas demais. Nesse contexto, $\mathbf{X}=\left(\mathbf{x}_{1}, \mathbf{x}_{2}, \ldots, \mathbf{x}_{n}\right)^{\prime}$ é composta por constantes conhecidas e, portanto, $\mathbf{X}$ não é uma matriz aleatória. Assim, cada $Y_{i}$ tem a distribuição de probabilidades de acordo com a Tabela 2.1.

\begin{tabular}{cl}
\hline \hline$Y_{i}$ & Probabilidade \\
\hline 1 & $P\left(Y_{i}=1 \mid \mathbf{x}_{i}\right)=\pi\left(\mathbf{x}_{i}\right)$ \\
0 & $P\left(Y_{i}=0 \mid \mathbf{x}_{i}\right)=1-\pi\left(\mathbf{x}_{i}\right)$ \\
\hline \hline
\end{tabular}

Tabela 2.1: Distribuição de probabilidades de $Y$.

Pela definição de esperança de variáveis aleatórias discretas, temos que:

$$
E\left[Y_{i} \mid \mathbf{x}_{i}\right]=\pi\left(\mathbf{x}_{i}\right), \quad i=1,2, \ldots, n
$$

ou seja, o valor esperado de $Y_{i}$ sempre representará a probabilidade de sucesso $\pi\left(\mathbf{x}_{i}\right)$.

Definiremos a seguir os tipos de covariáveis que podem estar presentes nesse modelo:

1. quantitativas: características que são numericamente mensuráveis, ou seja, que apresentam valores numéricos que fazem sentido;

2. qualitativas: características que se baseiam em modalidades e que não podem ser mensuradas numericamente.

Suponha que tenhamos $p_{q}$ covariáveis quantitativas e $p_{f}$ covariáveis qualitativas. Para construirmos o modelo de regressão logística, os dois tipos de covariáveis devem sofrer alterações. As covariáveis quantitativas, por exemplo, necessitam ser padronizadas de tal forma que a sua esperança seja igual a zero e a sua variância seja igual a um. Seja $\ddot{x}_{i j}$ o valor observado da j-ésima covariável para o i-ésimo elemento amostral e tal que a média da j-ésima covariável seja dada por:

$$
\bar{x}_{j}=\sum_{i=1}^{n} \frac{\ddot{x}_{i j}}{n}
$$

e a sua variância amostral seja igual a:

$$
s_{j}^{2}=\frac{\sum_{i=1}^{n}\left(\ddot{x}_{i j}-\bar{x}_{j}\right)}{n-1} .
$$

Assim, o valor padronizado da j-ésima covariável para o i-ésimo elemento amostral será igual a: 


$$
x_{i j}=\frac{\ddot{x}_{i j}-\bar{x}_{j}}{s_{j}} .
$$

Já uma covariável qualitativa com $c$ categorias deverá ser codificada com $c-1$ variáveis indicadoras ou variáveis dummy. Se o número de categorias das covariv́eis qualitativas é $c_{1}, c_{2}, \ldots, c_{p_{f}}$, então o número total de variáveis indicadoras necessárias será $\sum_{b=1}^{p_{f}} c_{b}-p_{f}$. Dessa forma, considere o seguinte modelo de regressão logística múltipla para o i-ésimo indivíduo:

$$
\begin{aligned}
\pi\left(\mathbf{x}_{i}\right) & =P\left(Y_{i}=1 \mid \mathbf{x}_{i}\right) \\
& =\frac{\exp \left(\beta_{0}+\beta_{1} x_{i 1}+\ldots+\beta_{p-1} x_{i p-1}\right)}{1+\exp \left(\beta_{0}+\beta_{1} x_{i 1}+\ldots+\beta_{p-1} x_{i p-1}\right)} \\
& =\frac{\exp \left(\mathbf{x}_{i}^{\prime} \beta\right)}{1+\exp \left(\mathbf{x}_{i}^{\prime} \beta\right)}
\end{aligned}
$$

em que $\pi\left(\mathbf{x}_{\mathbf{i}}\right)$ é a probabilidade de sucesso do evento de interesse para o i-ésimo elemento amostral, $Y_{i}$ é o valor da variável resposta binária do i-ésimo indivíduo definida como anteriormente e $\beta=\left(\beta_{0}, \beta_{1}, \ldots, \beta_{p-1}\right)^{\prime}$ é um vetor $p \times 1$ de parâmetros desconhecidos associados às covariáveis, $p=1+p_{q}+\sum_{b=1}^{p_{f}} c_{b}-p_{f}$ e $i=1,2, \ldots, n$.

Outra forma de representar o modelo de regressão logística é aplicando a transformação logito em (2.2), chegando na seguinte equação:

$$
\ln \left[\frac{\pi\left(\mathbf{x}_{i}\right)}{1-\pi\left(\mathbf{x}_{i}\right)}\right]=\beta_{0}+\beta_{1} x_{1 i}+\ldots+\beta_{p-1} x_{i p-1}=\mathbf{x}_{i}^{\prime} \beta .
$$

A expressão dada por (2.3) é conhecida como logaritmo da chance do i-ésimo elemento apresentar o evento de interesse.

Para exemplificar os vetores descritos em (2.2) e em (2.3), considere o conjunto de dados do estudo ICU utilizado em Hosmer e Lemeshow (2000). Este conjunto de dados é composto de 200 pacientes internados em uma unidade de terapia intensiva (ICU, Intensive Care Unit) e o principal objetivo do estudo era prever a probabilidade de sobrevivência destes pacientes, ou seja, a variável resposta era dada por:

$$
Y_{i}= \begin{cases}1, & \text { se o i-ésimo paciente sobreviveu } \\ 0, & \text { caso contrário. }\end{cases}
$$

Para prever a sobrevivência dos pacientes foram utilizadas covariáveis como idade padronizada do paciente, denominada $\mathbf{x}_{1}$, gênero do paciente (masculino ou feminino), raça do paciente (branca, negra ou outra) e nível de consciência do paciente no momento da internação (não estava em coma nem em sono profundo, estava em sono profundo ou estava em coma). As covariáveis qualitativas tiveram que ser transformadas em variáveis indicadoras 
da seguinte forma:

$$
\begin{gathered}
\mathbf{x}_{2}= \begin{cases}1, & \text { se o i-ésimo paciente é do gênero feminino } \\
0, & \text { caso contrário. }\end{cases} \\
\mathbf{x}_{3}= \begin{cases}1, & \text { se o i-ésimo paciente é da raça negra } \\
0, & \text { caso contrário. }\end{cases}
\end{gathered}
$$$$
\mathbf{x}_{4}= \begin{cases}1, & \text { se o i-ésimo paciente não é da raça branca e nem da negra } \\ 0, & \text { caso contrário. }\end{cases}
$$

$\mathbf{x}_{5}= \begin{cases}1, & \text { se o i-ésimo paciente estava em sono profundo no momento da internação } \\ 0, & \text { caso contrário. }\end{cases}$

$$
\mathbf{x}_{6}= \begin{cases}1, & \text { se o i-ésimo paciente estava em coma no momento da internação } \\ 0, & \text { caso contrário. }\end{cases}
$$

Assim, o vetor $\mathbf{x}_{i}$ terá sete componentes $(p=1+1+8-3=7)$, sendo que o primeiro componente será igual a 1 , o segundo será destinado à idade padronizada do paciente e os demais serão de acordo com as variáveis indicadoras apresentadas anteriormente.

Se selecionarmos, por exemplo, o paciente com código de inscrição igual a 8 temos que ele sobreviveu, tem 27 anos, é do sexo feminino, da raça branca e não estava em coma nem em sono profundo no momento da internação. O valor da covariável relacionada à idade deverá, primeiramente, ser padronizada. A média e o desvio padrão são iguais a 57,545 e 20,055, respectivamente. Portanto, temos que o valor da idade padronizada para esse paciente será:

$$
x_{1}=\frac{27-57,545}{20,055}=-1,523 .
$$

Dessa forma, os vetores definidos anteriormente relacionados à esse paciente ficarão iguais a:

$$
Y_{8}=1, \quad \mathbf{x}_{8}=\left(\begin{array}{llllllll}
1 & -1,523 & 1 & 0 & 0 & 0 & 0
\end{array}\right), \quad \beta=\left(\begin{array}{c}
\beta_{0} \\
\beta_{1} \\
\beta_{2} \\
\beta_{3} \\
\beta_{4} \\
\beta_{5} \\
\beta_{6}
\end{array}\right) .
$$

Exposto o modelo, comentaremos a seguir as suas suposições. A análise de regressão logística é regida pelas mesmas linhas de análise da regressão linear, apesar de seus modelos não 
terem as mesmas suposições de normalidade e variância constante para a variável resposta. Além disso, a variável resposta do modelo de regressão logística é binária e os resultados inferenciais utilizados na análise são todos assintóticos, enquanto que na regressão linear eles são exatos. Na Tabela 2.2 estão ilustradas as diferenças entre os dois tipos de modelos.

\begin{tabular}{lcc}
\hline \hline Medida & Regressão Linear & Regressão Logística \\
\hline Modelo & $y_{i}^{*}=\left(\mathbf{x}_{i}^{*}\right)^{\prime} \beta^{*}+\epsilon_{i}^{*}$ & $y_{i}=\pi\left(\mathbf{x}_{i}\right)+\epsilon_{i}$ \\
Distribuição da resposta & $y_{i}^{*} \sim N\left(\left(\mathbf{x}_{i}^{*}\right)^{\prime} \beta^{*}, \sigma^{2}\right)$ & $y_{i} \sim \operatorname{Bernoulli}\left(\pi\left(\mathbf{x}_{i}\right)\right)$ \\
Esperança da resposta & $\mathrm{E}\left[Y_{i}^{*} \mid \mathbf{x}_{i}^{*}\right]=\left(\mathbf{x}_{i}^{*}\right)^{\prime} \beta^{*}$ & $\mathrm{E}\left[Y_{i} \mid \mathbf{x}_{i}\right]=\pi\left(\mathbf{x}_{i}\right)$ \\
Limites da esperança & $-\infty<\mathrm{E}\left[Y_{i}^{*} \mid \mathbf{x}_{i}^{*}\right]<\infty$ & $0 \leq \mathrm{E}\left[Y_{i} \mid \mathbf{x}_{i}\right] \leq 1$ \\
da resposta & $\epsilon_{i}^{*} \sim N\left(0, \sigma^{2}\right)$ & $\mathrm{E}\left[\epsilon_{i}\right]=0, \operatorname{Var}\left[\epsilon_{i}\right]=\pi\left(\mathbf{x}_{i}\right)\left(1-\pi\left(\mathbf{x}_{i}\right)\right)$ \\
Distribuição dos erros
\end{tabular}

Tabela 2.2: Diferenças entre o modelo de regressão linear e o modelo de regressão logística.

Portanto, temos que no modelo de regressão logística:

1. A distribuição dos erros não é Normal, pois, para uma variável binária, cada erro $\epsilon_{i}$ irá assumir apenas dois valores: $1-\pi\left(\mathbf{x}_{i}\right)$, com probabilidade $\pi\left(\mathbf{x}_{i}\right) ;$ ou $-\pi\left(\mathbf{x}_{i}\right)$, com probabilidade $1-\pi\left(\mathbf{x}_{i}\right)$. No modelo de regressão linear, considera-se erros normalmente distribuídos;

2. As variâncias dos erros não são constantes para todo $\mathbf{x}_{j}$, não valendo, portanto, $\mathbf{a}$ suposição de homocedasticidade;

3. A quantidade $E\left[Y_{i} \mid \mathbf{x}_{i}\right]$ é uma probabilidade, ou seja, pertence ao intervalo $[0,1]$.

\subsection{Estimação dos parâmetros}

Nos modelos de regressão logística o método comumente empregado na estimação dos parâmetros é o de máxima verossimilhança. Sem perda de generalidade, vamos considerar que $\pi\left(\mathbf{x}_{i}\right)=\pi_{i}$ é a probabilidade de sucesso do evento para o i-ésimo elemento amostral. Para uma amostra de tamanho $n$, temos que a função densidade de probabilidade conjunta ou função de verossimilhança de $\mathbf{Y}=\left(Y_{1}, Y_{2}, \ldots, Y_{n}\right)^{\prime}$, que é um vetor $n \times 1$ com o valor da variável resposta binária dos $n$ indivíduos, é dada por:

$$
L\left(\beta_{0}, \beta_{1}, \ldots, \beta_{p-1} \mid y_{1}, \ldots, y_{n}\right)=\prod_{i=1}^{n} f\left(y_{i}\right)=\prod_{i=1}^{n} \pi_{i}^{y_{i}}\left(1-\pi_{i}\right)^{1-y_{i}}
$$

já que se assume que as variáveis aleatórias $Y_{i}, i=1, \ldots, n$, têm distribuição $\operatorname{Bernoulli}\left(\pi_{i}\right)$ e são independentes entre si. 
Aplicando-se o logaritmo neperiano a ambos os lados da equação (2.4), obtemos:

$$
\ln \left(L\left(\beta_{0}, \beta_{1}, \ldots, \beta_{p-1} \mid y_{1}, \ldots, y_{n}\right)\right)=\sum_{i=1}^{n} y_{i} \ln \left(\frac{\pi_{i}}{1-\pi_{i}}\right)+\sum_{i=1}^{n} \ln \left(1-\pi_{i}\right) .
$$

Substituindo a equação (2.3) na equação (2.5), temos:

$$
\begin{aligned}
\ell(\beta)= & \sum_{i=1}^{n} y_{i}\left(\beta_{0}+\beta_{1} x_{i 1}+\ldots+\beta_{p-1} x_{i p-1}\right)- \\
& -\sum_{i=1}^{n} \ln \left[1+\exp \left(\beta_{0}+\beta_{1} x_{i 1}+\ldots+\beta_{p-1} x_{i p-1}\right)\right]
\end{aligned}
$$

sendo que $\ell(\beta)=\ln \left(L\left(\beta_{0}, \beta_{1}, \ldots, \beta_{p-1} \mid y_{1}, \ldots, y_{n}\right)\right)$. Para se obter as estimativas de máxima verossimilhança dos parâmetros do modelo, precisamos derivar a equação (2.6) em relação a cada um dos parâmetros e igualar a zero, ou seja, matricialmente teremos:

$$
\frac{\partial \ell(\beta)}{\partial \beta}=\mathbf{X}^{\prime}(\mathbf{Y}-\pi)=\mathbf{0}
$$

em que $\mathbf{X}=\left(\mathbf{1}, \mathbf{x}_{\mathbf{1}}, \ldots, \mathbf{x}_{\mathbf{p}-\mathbf{1}}\right)$ é a matriz de planejamento $n \times \mathrm{x} p$ composta do vetor $n \mathrm{x} 1$ de 1 's, denotado por $\mathbf{1}$, em sua primeira coluna e dos valores padronizados das covariáveis associadas aos $n$ elementos amostrais, ou seja, $\mathbf{x}_{\mathbf{j}}$ é um vetor $n \times 1$ contendo o valor padronizado da $\mathbf{j}$ ésima covariável para os $n$ indivíduos, $j=1, \ldots, p$-1, e $\mathbf{0}$ é o vetor nulo com $n$ componentes.

Como $\hat{\pi}=\hat{\mathbf{Y}}$, segue que:

$$
\mathbf{X}^{\prime}(\mathbf{Y}-\widehat{\mathbf{Y}})=\mathbf{0}
$$

Podemos verificar que a equação (2.7) não é linear em $\beta$ e que não é possível obter a solução explícita desse sistema. Por isso, temos que recorrer a métodos iterativos para calcular as estimativas dos parâmetros. Um dos procedimentos mais utilizados é o algoritmo de Newton-Raphson, que leva em consideração a matriz de informação observada, ou seja, a segunda derivada da função de verossimilhança em relação aos parâmetros. Para maiores informações sobre este e outros métodos iterativos, ver Hilbe (2009). Através do método de Newton-Raphson, portanto, a equação (2.7) pode ser resolvida de maneira iterativa, chegando-se ao seguinte estimador de $\beta$ :

$$
\hat{\beta}=\left(\mathbf{X}^{\prime} \hat{\mathbf{W}} \mathbf{X}\right)^{-\mathbf{1}} \mathbf{X}^{\prime} \hat{\mathbf{W}} \mathbf{Z}
$$

com $\hat{\mathbf{W}}$ sendo a matriz diagonal $n \mathrm{x} n$ com elementos $\hat{\pi}_{i}\left(1-\hat{\pi}_{i}\right), i=1, \ldots, n, \hat{\pi}_{i}$ o estimador de máxima verossimilhança de $\pi_{i}$ e $\mathbf{Z}$ é um vetor $n \times 1$ com elementos dados por:

$$
Z_{i}=\ln \left(\frac{\hat{\pi}_{i}}{1-\hat{\pi}_{i}}\right)+\frac{Y_{i}-\hat{\pi}_{i}}{\hat{\pi}_{i}\left(1-\hat{\pi}_{i}\right)}
$$


Se a equação (2.9) for reescrita de forma a mostrar a atualização de $\hat{\beta}$ em cada etapa do processo iterativo, na etapa $l$ teremos:

$$
\mathbf{Z}^{(\mathbf{l})}=\mathbf{X} \hat{\beta}^{(\mathbf{l})}+\left(\hat{\mathbf{W}}^{(l)}\right)^{-1}\left(\mathbf{Y}-\hat{\pi}^{(l)}\right)
$$

em que $\mathbf{Z}^{(l)}, \hat{\beta}^{(l)}, \hat{\mathbf{W}}^{(l)}$ e $\hat{\pi}^{(l)}$ são os valores de $\mathbf{Z}$, da estimativa de $\beta$, da estimativa de $\mathbf{W}$ e da estimativa de $\pi$ avaliada na $l$-ésima etapa do processo iterativo, respectivamente, e $\pi$ é um vetor $n \times 1$ contendo os valores de $\pi_{i}, i=1, \ldots, n$.

Portanto, o estimador de $\beta$ na l-ésima etapa do processo será dado por:

$$
\hat{\beta}^{(\mathbf{l}+\mathbf{1})}=\hat{\beta}^{(\mathbf{l})}+\left(\mathbf{X}^{\prime} \hat{\mathbf{W}}^{(\mathbf{l})} \mathbf{X}\right)^{-\mathbf{1}} \mathbf{X}^{\prime}\left(\mathbf{Y}-\hat{\pi}^{(\mathbf{l})}\right) .
$$

O processo dado pela equação (2.10) se repete até a convergência da estimativa.

Para se fazer inferências, utiliza-se o fato que $\hat{\beta}$ converge em distribuição para uma distribuição normal multivariada de vetor de médias $\beta$ e matriz de variância e covariância aproximada por:

$$
\widehat{\operatorname{Var}}(\hat{\beta})=\left(\mathbf{X}^{\prime} \hat{\mathbf{W}} \mathbf{X}\right)^{-\mathbf{1}}
$$

\subsection{Teste de significância dos parâmetros}

Após ajustado o modelo de regressão logística, temos que dar início à avaliação do modelo, e um dos primeiros passos é a construção de testes de hipóteses para os seus parâmetros com o intuito de verificar a importância das covariáveis. De modo geral, deseja-se verificar se o modelo que contem uma covariável ou um conjunto de covariáveis em questão consegue explicar melhor a variável resposta binária do que o modelo sem essa covariável ou sem esse conjunto de covariáveis. Esse objetivo é alcançado comparando os valores observados da variável resposta com os valores ajustados por esses dois modelos. Em modelos de regressão logística essa comparação é feita através do logaritmo da função de verossimilhança dada por (2.6).

De acordo com Hosmer e Lemeshow (2000), podemos calcular a estatística do teste univariado de Wald para testar se um parâmetro é diferente de zero individualmente. Para testar a hipótese nula de que $\beta_{j}$ é igual a zero, $j=1, \ldots, p-1$, temos que calcular a seguinte estatística:

$$
\operatorname{Wald}_{j}=\frac{\hat{\beta}_{j}}{\sqrt{\widehat{\operatorname{Var}}\left(\hat{\beta}_{j}\right)}}
$$

Sob a hipótese nula de que $\beta_{j}$ é igual a zero, a estatítica (2.12) segue uma distribuição normal padrão.

Além disso, podemos utilizar o teste da razão de verossimilhanças para testar a hipótese 
nula de que um grupo de parâmetros (desconsiderando a constante do modelo) conjuntamente são iguais a zero. Com esse teste podemos verificar se os parâmetros referentes a todas as covariáveis ou somente a um conjunto de $q$ covariáveis são nulos. A estatística deste teste é dada por:

$$
G=-2 \ln \left[\frac{\left(\sum_{i=1}^{n} \frac{Y_{i}}{n}\right)^{\sum_{i=1}^{n} Y_{i}}\left(\sum_{i=1}^{n} \frac{1-Y_{i}}{n}\right)^{\sum_{i=1}^{n}\left(1-Y_{i}\right)}}{\prod_{i=1}^{n} \hat{\pi}_{i}^{Y_{i}}\left(1-\hat{\pi}_{i}\right)^{\left(1-Y_{i}\right)}}\right]
$$

em que $\hat{\pi}_{i}$ é o valor ajustado da variável resposta considerando todas ou somente as $q$ covariáveis, dependendo do caso que se quer testar. A estatística de teste definida em (2.13), portanto, é igual a menos duas vezes a diferença do logaritmo da função de verossimilhança do modelo somente com a constante em relação ao logaritmo da função de verossimilhança do modelo saturado, ou seja, com todas as $p$-1 covariáveis, ou com as $q$ covariáveis. Sob a hipótese nula de que todos os parâmetros exceto a constante do modelo são iguais a zero, a estatística (2.13) segue uma distribuição $\chi^{2}$ com $p$-1 graus de liberdade. Já sob a hipótese nula de que todos os $q$ parâmetros exceto a constante do modelo são iguais a zero, a estatística (2.13) segue uma distribuição $\chi^{2}$ com $q$ graus de liberdade.

Em muitos softwares estatísticos o teste de razão de verossimilhanças é utilizado com uma forma mecânica de redução do número de covariáveis do modelo. Esse método é conhecido como stepwise e é uma ferramenta muito rápida e eficaz na seleção ou exclusão de variáveis baseado em algoritmos estatísticos que checam a importância das mesmas com base em alguma regra de decisão previamente fixada. Mais detalhes a respeito desse assunto podem ser encontrados no Apêndice A. Contudo, de acordo com Paula (1999), este método pode levar a um modelo sem sentido e de difícil interpretação, pois, muitas vezes covariáveis consideradas como importantes não deveriam ser deixadas de lado pela sua falta de significância estatística. Assim, a seleção de um modelo logístico deve ser um processo conjugado de seleção estatística de modelos e bom senso.

\subsection{Avaliação do ajuste do modelo}

Depois de terem sido feitos todos os testes de significância dos parâmetros e termos chegado em um modelo em que todas as covariáveis são importantes na predição da variável resposta, passamos então a analisar a qualidade do ajuste do modelo final através da análise de resíduos.

Uma possível maneira de se avaliar a qualidade do ajuste do modelo é através do cálculo de uma medida que leva em consideração a diferença entre o valor observado de $\mathbf{Y}$ e o seu valor ajustado $\hat{\mathbf{Y}}$. Se a discrepância desses valores for pequena, então haverá uma indicação de que o modelo está bem ajustado aos dados. Caso contrário, haverá uma clara indicação da existência de problemas com o modelo.

Para alcançar este objetivo, proporemos a seguir o teste de Hosmer-Lemeshow, presente 
em Lemeshow e Hosmer (1982) e Hosmer e Lemeshow (1980), artigos citados em Hosmer e Lemeshow (2000). Nele, são formados grupos baseados nos valores estimados das probabilidades $\pi(\mathbf{x})=$ $P(Y \mid \mathbf{x})$ segundo um dos seguintes critérios:

1. percentis das probabilidades estimadas $\hat{\pi}(\mathbf{x})$ ou

2. valores fixos das probabilidades estimadas.

Com o primeiro método, os autores sugerem a formação de $g$ grupos de mesmo tamanho (aproximadamente) da seguinte forma:

- Grupo 1: formado pelos $n_{1}=\frac{n}{g}$ elementos amostrais com os menores valores de $\hat{\pi}(\mathbf{x})$;

- Grupo 2: formado pelos $n_{2}=\frac{n}{g}$ elementos amostrais com os segundos menores valores de $\hat{\pi}(\mathbf{x})$;

e, assim, sucessivamente até a obtenção do g-ésimo grupo:

- Grupo g: formado pelos $n_{g}=\frac{n}{g}$ elementos amostrais com os maiores valores de $\hat{\pi}(\mathbf{x})$.

Quando não há empates de probabilidades estimadas, a quantidade $\frac{n}{g}$ fica fácil de ser calculada. Caso contrário, será necessário alocar os elementos amostrais com igual probabilidade no mesmo grupo.

Com o segundo método, os autores sugerem a formação de $g$ grupos com pontos de corte para $\hat{\pi}(\mathbf{x})$ iguais a $\frac{1}{g}, \frac{2}{g}, \ldots, \frac{g-1}{g}$. Assim teremos:

- Grupo 1: formado pelos elementos amostrais com $0 \leq \hat{\pi}(\mathbf{x}) \leq \frac{1}{g}$;

- Grupo 2: formado pelos elementos amostrais $\operatorname{com} \frac{1}{g}<\hat{\pi}(\mathbf{x}) \leq \frac{2}{g}$;

e, assim, sucessivamente até a obtenção do g-ésimo grupo:

- Grupo g: formado pelos elementos amostrais com $\frac{g-1}{g}<\hat{\pi}(\mathbf{x}) \leq 1$.

Como dificilmente a probabilidade do evento estudado alcança 100\%, então propomos que as probabilidades limites dos grupos desse segundo método sejam funções da probabilidade máxima estimada do evento, ou seja, se denominarmos por $\hat{\pi}(\mathbf{x})_{\max }$ a probabilidade máxima estimada do evento, então os grupos ficarão dados por:

- Grupo 1: formado pelos elementos amostrais com $0 \leq \hat{\pi}(\mathbf{x}) \leq \frac{\hat{\pi}(\mathbf{x})_{\max }}{g}$;

- Grupo 2: formado pelos elementos amostrais $\operatorname{com} \frac{\hat{\pi}(\mathbf{x})_{\max }}{g}<\hat{\pi}(\mathbf{x}) \leq \frac{2 \cdot \hat{\pi}(\mathbf{x})_{\max }}{g}$; e, assim, sucessivamente até a obtenção do $g$-ésimo grupo:

- Grupo g: formado pelos elementos amostrais $\operatorname{com} \frac{(g-1) \cdot \hat{\pi}(\mathbf{x})_{\max }}{g}<\hat{\pi}(\mathbf{x}) \leq \hat{\pi}(\mathbf{x})_{\max }$. 
Independente do método utilizado para obtenção dos grupos, os autores sugerem que a quantidade de grupos seja igual a 10.

Para cada método, é obtido um valor da estatística do teste de Hosmer-Lemeshow, denominada $\hat{C}$, com o objetivo de avaliar a qualidade do ajuste do modelo. Este teste tem como base a estatística $\chi^{2}$ de Pearson, ou seja, calcula as diferenças entre o valor observado da variável resposta e o seu valor estimado, eleva ao quadrado, pondera pelo o seu valor estimado e soma para todos os elementos amostrais. Assim, a fórmula para o cálculo de $\hat{C}$ é dada por:

$$
\hat{C}=\sum_{l=1}^{g} \frac{\left(o_{l}-n_{l} \bar{\pi}_{l}\right)^{2}}{n_{l} \bar{\pi}_{l}\left(1-\bar{\pi}_{l}\right)}
$$

em que $o_{l}=\sum_{i=1}^{n_{l}} y_{i}$, ou seja, é o número de elementos amostrais que apresentaram o evento de interesse no $l$-ésimo grupo, $n_{l}$ é o número de elementos amostrais no $l$-ésimo grupo e $\bar{\pi}_{l}=\sum_{i=1}^{n_{l}} \frac{\hat{\pi}_{i}}{n_{l}}$ é a média das probabilidades estimadas do evento dentro do l-ésimo grupo.

Hosmer e Lemeshow (1980) verificaram através de simulações que, dentro de certas condições, a distribuição assintótica da estatística $\hat{C}$ pode ser bem aproximada por uma distribuição $\chi^{2}$ com $g$-2 graus de liberdade.

Outra forma de se analisar a qualidade do ajuste é através da matriz de classificação ou confusão. Segundo Hilbe (2009), essa matriz foi um dos primeiros métodos utilizados com esse propósito por estatísticos na regressão logística. A matriz de classificação é baseada em um ponto de corte, que responde qual a probabilidade ótima para separar o sucesso do fracasso dentre os valores preditos, e em dois conceitos denominados sensibilidade e especificidade. Para exemplificar, suponha que temos pacientes portadores de uma determinada doença, $D$, e pacientes que não são portadores desta doença, $\bar{D}$. Se fizermos um teste para determinar se o paciente tem ou não esta doença, temos que a probabilidade deste teste ser positivo para a doença dado que o paciente é portador da mesma, é denominada sensibilidade do teste. Já a probabilidade dele ser negativo para a doença dado que o paciente não é portador da mesma, é denominada especificidade do teste. Portanto:

$$
\begin{aligned}
\text { Sensibilidade } & =P(\text { teste ser positivo } \mid D) \\
\text { Especificidade } & =P(\text { teste ser negativo } \mid \bar{D})
\end{aligned}
$$

O ponto de corte usual é o 0,50, que indica a média da distribuição logística. Entretanto, este não é um valor apropriado para a maioria dos modelos de regressão logística. O problema, então, está em definir o melhor ponto de corte em que a classificação estará correta para os dados e para o modelo. Pensando dessa forma, um bom candidato para este valor é a média da variável resposta. Como a variável resposta é 0 ou 1 e o valor ajustado será uma probabilidade, a ideia é utilizar o ponto de corte como o proporção de resposta. Portanto, 
classificaremos os indivíduos da seguinte maneira:

- Se $\hat{y}_{i} \geq \bar{\pi}$, então o classificamos como um sucesso $\left(\hat{y}_{i}^{C}=1\right)$

- Se $\hat{y}_{i}<\bar{\pi}$, então o classificamos como um fracasso $\left(\hat{y}_{i}^{C}=0\right)$

em que $\bar{\pi}=\frac{1}{n} \sum_{i=1}^{n} y_{i}$ é o ponto de corte e a proporção da resposta, $i=1, \ldots, n$. Dessa forma, conseguimos construir uma tabela $2 \times 2$, em que teremos nas linhas os possíveis valores observados da resposta $\left(y_{i}=10 u 0\right)$ e nas colunas os possíveis valores classificados através da variável resposta predita $\left(\hat{y}_{i}^{C}=1 o u 0\right)$. A Figura 2.1 ilustra a matriz de classificação.

\begin{tabular}{|c|c|c|c|c|}
\hline \multicolumn{2}{|c|}{} & \multicolumn{2}{c|}{ Predito } & \multirow{2}{*}{ Total } \\
\cline { 3 - 5 } \multicolumn{2}{|c|}{} & $\hat{y}_{i}^{C}=0$ & $\hat{y}_{i}^{C}=1$ & \\
\hline \hline \multirow{2}{*}{ Observado } & $y_{i}=0$ & $n_{00}$ & $n_{01}$ & $n_{0 .}$ \\
\cline { 2 - 5 } & $y_{i}=1$ & $n_{10}$ & $n_{11}$ & $n_{1 .}$ \\
\hline \hline \multicolumn{2}{|c|}{ Total } & $n_{.0}$ & $n_{.1}$ & $\mathrm{n}$ \\
\hline
\end{tabular}

Figura 2.1: Matriz de classificação.

As quantidades $n_{00}$ e $n_{11}$ representam as quantidades de indivíduos que foram classificados corretamente como fracasso e sucesso, respectivamente, ou seja, eles fracassaram e foi predito um fracasso ou eles tiveram um sucesso e foi predito um sucesso. Já as quantidades $n_{01}$ e $n_{10}$ representam as quantidades de indivíduos que foram classificados erroneamente, ou seja, no primeiro caso eles fracassaram mas foi predito um sucesso e no segundo caso eles tiveram um sucesso mas foi predito um fracasso. As quantidades $n_{0 .}, n_{1 .}, n_{.0}$ e $n_{.1}$ representam, respectivamente, as quantidades totais de fracassos e sucessos observados e fracassos e sucessos preditos.

Uma forma mais eficiente de demonstrar a relação entre a sensibilidade e a especificidade é a curva ROC (Receiver Operator Characteristic). Esta curva é uma ferramenta poderosa para medir e especificar problemas no desempenho do diagnóstico por permitir estudar a variação da sensibilidade e especificidade para diversos valores de corte. A curva ROC é um gráfico em que é plotado a sensibilidade, ou seja, o percentual de indivíduos que foram classificados corretamente como sucesso, versus um menos a especificidade, ou seja, o percentual de indivíduos que foram classificados erroneamente como sucesso. A área abaixo desta curva, que pode variar de 0 a 1, fornece uma medida de discriminação entre os indivíduos que apresentaram a característica de interesse versus aqueles que não apresentaram. Na Figura 2.2 é mostrado um exemplo da curva ROC.

De maneira geral, segundo Hosmer e Lemeshow (1980), podemos classificar a discriminação do modelo como mostrado na Tabela 2.3. 


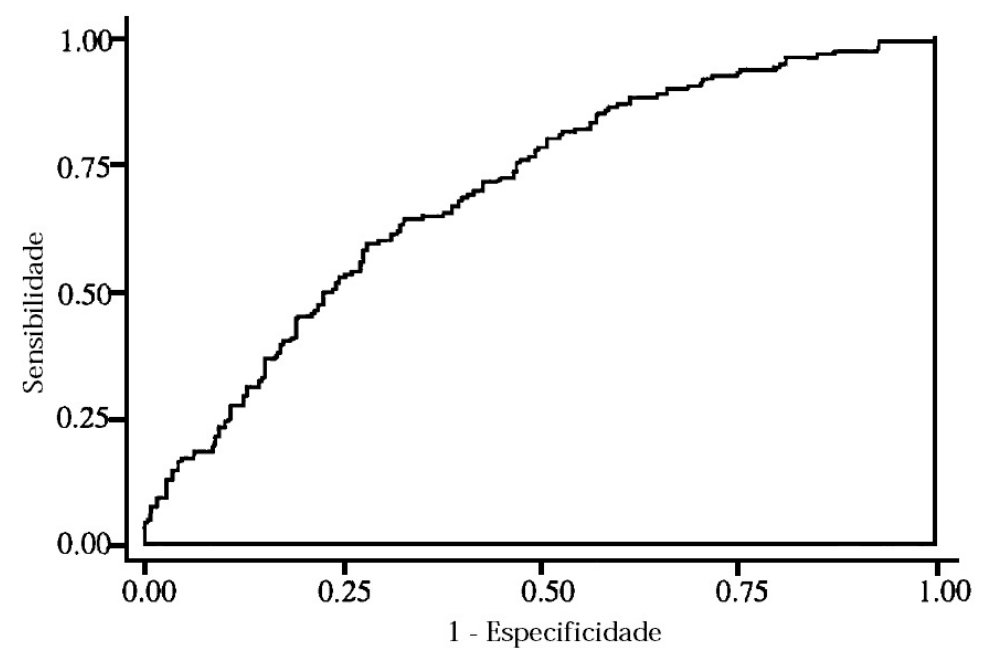

Figura 2.2: Exemplo da curva ROC.

\begin{tabular}{cc}
\hline \hline Valor da área abaixo da curva ROC & Discriminação do modelo \\
\hline$<0,7$ & Baixa \\
{$[0,7 ; 0,8[$} & Aceitável \\
{$[0,8 ; 0,9[$} & Muito bom \\
$\geq 0,9$ & Excelente \\
\hline \hline
\end{tabular}

Tabela 2.3: Classificação do poder de discriminação do modelo segundo a área abaixo da curva $R O C$.

\subsection{Considerações finais do capítulo}

Neste capítulo foi apresentado o modelo de regressão logística que será utilizado no presente trabalho, bem como o processo para estimação através de estimadores assintoticamente não viesados. Foi utilizado o estimador de máxima verossimilhança e apresentados testes para determinar a significância dos parâmetros e alguns procedimentos para avaliar a qualidade de ajuste do modelo. Vamos, a seguir, apresentar o conceito de multicolinearidade e suas consequências em modelos de regressão logística. Introduziremos também algumas técnicas para identificar este problema. 


\section{Capítulo 3}

\section{Efeitos e Diagnóstico da}

\section{Multicolinearidade nos Modelos de Regressão Logística}

O uso e a interpretação dos modelos de regressão logística normalmente dependem direta ou indiretamente das estimativas dos seus coeficientes. Se não existe relação linear entre as covariáveis do modelo, ou seja, se elas forem ortogonais, inferências como mensuração dos efeitos das covariáveis na variável dependente e predição podem ser realizadas. Porém, na maioria dos casos, infelizmente as covariáveis não são ortogonais e dizemos que o problema da multicolinearidade existe. Consequentemente, a inferência baseada nessas estimativas pode estar seriamente comprometida.

Este capítulo tem o objetivo de definir a multicolinearidade, avaliar os seus efeitos e apresentar técnicas utilizadas no seu diagnóstico em modelos de regressão logística.

\subsection{Efeitos da multicolinearidade}

Na prática, de acordo com Schaefer (1986), existe multicolinearidade no modelo de regressão logística quando há uma dependência linear exata ou aproximada entre as covariáveis do modelo. Geometricamente, a multicolinearidade perfeita existe quando a matriz $\mathbf{X}$ tem posto não completo. Vamos mostrar agora que, dependendo do nível de associação entre as covariáveis, essa característica da matriz de planejamento pode fazer com que os estimadores fiquem imprecisos e pode afetar a inferência associada aos parâmetros. Além disso, apresentaremos a relação deste problema entre os modelos de regressão linear e os de regressão logística. 


\subsubsection{Decomposição em valores singulares da matriz de planeja- mento}

Antes de iniciarmos a discussão sobre multicolinearidade nos modelos de regressão linear e logística, vamos introduzir uma técnica que será muito utilizada daqui para frente: a Decomposição em Valores Singulares (DVS) de uma matriz. O objetivo dessa técnica é, segundo Mandel (1982) e Eubank e Webstern (1985), reorientar os eixos de coordenadas, de maneira a torná-los mais próximos ao padrão dos dados. Veremos a seguir que a DVS auxilia no entendimento da estrutura da matriz.

Seja $\hat{\mathbf{K}}$ uma matriz $n \times \mathrm{x} p$ tal que seu posto é dado por $\mathrm{r}(\hat{\mathbf{K}})=s \leq p<n$. A matriz $\hat{\mathbf{K}}$ pode ser, por exemplo, uma matriz de planejamento do modelo de regressão linear ou logística e a técnica estabelece que esta matriz pode ser decomposta como:

$$
\hat{\mathbf{K}}=\mathbf{U D V}^{\prime}
$$

em que U é uma matriz $n \times x$ cujas colunas são os autovetores $\nu_{j}$ de $\hat{\mathbf{K}} \hat{\mathbf{K}}^{\prime}$ tal que $\mathbf{U}^{\prime} \mathbf{U}=\mathbf{I}_{\mathbf{s}}$, D é uma matriz diagonal $s \mathrm{x} s$ composta da raiz quadrada dos autovalores não nulos de $\hat{\mathbf{K}}^{\prime} \hat{\mathbf{K}}$, denominados $\mu_{j}$ e tais que $\mu_{0} \geq \mu_{1} \geq \ldots \geq \mu_{s-1}$, e $\mathbf{V}^{\prime}$ é uma matriz $s \times p$ cujas colunas são os autovetores de $\hat{\mathbf{K}}^{\prime} \hat{\mathbf{K}}$ associados a $\mu_{j}$, denominados $v_{j}$, de modo que $\mathbf{V}^{\prime} \mathbf{V}=\mathbf{I}_{\mathbf{s}}, j=0, \ldots, s-1$. A quantidade $\mu_{j}$ também pode ser denominada como o i-ésimo valor singular da matriz $\hat{\mathbf{K}}$.

Note que, em (3.1), a matriz $\hat{\mathbf{K}}$ foi decomposta de forma que os vetores que compoẽm a matriz $\mathbf{U}, \nu_{j}, j=0, \ldots, s-1$, são ortogonais em relação aos demais e de comprimento unitário, assim como os vetores que compõe a matriz $\mathbf{V}, v_{j}, j=0, \ldots, s-1$.

Para facilitar a interpretação geométrica da DVS vamos considerar um exemplo baseado em Mandel (1982). Suponha que existem somente duas covariáveis e cinco observações no modelo de regressão logística sem intercepto. A matriz de planejamento do modelo, $\hat{\mathbf{K}}$, assim como as matrizes $\mathbf{U}, \mathbf{D}$ e $\mathbf{V}^{\prime}$ encontram-se na Tabela 3.1.

\begin{tabular}{rcccc}
\hline \hline & \multicolumn{2}{c}{$\hat{\mathbf{K}}$} & \multicolumn{2}{c}{$\mathbf{U}$} \\
& $\hat{\mathbf{K}}_{\mathbf{0}}$ & $\hat{\mathbf{K}}_{\mathbf{1}}$ & $\nu_{0}$ & $\nu_{1}$ \\
\hline 1,3 & 1,2 & 0,1202 & 0,4037 \\
4,2 & 2,8 & 0,2511 & $-0,5113$ \\
6,3 & & 7,4 & 0,4867 & 0,6912 \\
8,0 & 7,1 & 0,5391 & $-0,1689$ \\
& 9,4 & 8,2 & 0,6285 & $-0,2634$ \\
\hline \hline \multicolumn{3}{c}{$\mathbf{V}^{\prime}$} & \multicolumn{3}{c}{$\mathbf{D}$} \\
\hline$v_{0}^{\prime}$ & 0,7309 & 0,6825 & 19,8360 & 0 \\
$v_{1}^{\prime}$ & $-0,6825$ & 0,7309 & 0 & 1,6040 \\
\hline \hline
\end{tabular}

Tabela 3.1: DVS da matriz de planejamento com duas covariáveis.

Podemos interpretar cada linha da matriz $\hat{\mathbf{K}}$, ou seja, cada par $\left(\hat{K}_{i 0}, \hat{K}_{i 1}\right)$, como um ponto em um espaço bidimensional de coordenadas $\hat{K}_{i 0}$ e $\hat{K}_{i 1}, i=1, \ldots, 5$. As linhas de $\mathbf{V}^{\prime}$ também 
representam pontos nesse mesmo espaço bidimensional, porém, mais do que isso, as retas que unem esses pontos à origem formam um conjunto alternativo de eixos ortogonais. Note também que a distância de $v_{0}^{\prime}$ e $v_{1}^{\prime}$ em relação à origem é igual a 1. Essas características podem ser observadas na Figura 3.1.

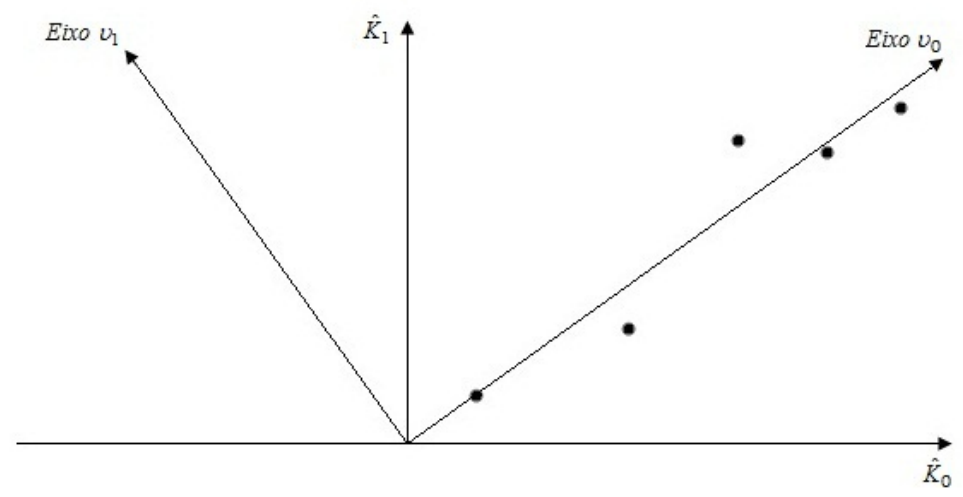

Figura 3.1: Interpretação geométrica da DVS no caso de duas covariáveis.

As projeções dos cinco pontos correspondentes às linhas da matriz $\hat{\mathbf{K}}$ no eixo alternativo $v_{0}$ são dadas por $\mu_{0} \cdot \nu_{0}$ e as projeções no eixo $v_{1}$ são dadas por $\mu_{1} \cdot \nu_{1}$. Então, a primeira observação dada por $(1,3,1,2)$ projetada no novo eixo ficará 19, 8360 • 0, $1202=2,3843 \mathrm{em}$ $v_{0}$ e $1,604 \cdot 0,4037=0,6475 \mathrm{em} v_{1}$. Na Tabela 3.2 se encontram as projeções das cinco observações. Como se pode perceber, a variação das projeções no eixo $v_{0}$ é muito superior ao do eixo $v_{1}$, indicando que somente o eixo $v_{0}$ já explicaria bastante o padrão dos dados.

\begin{tabular}{cc}
\hline \hline$\hat{\mathbf{K}}$ & $\mathbf{V}$ \\
\hline$(1,3,1,2)$ & $(2,38,0,65)$ \\
$(4,2,2,8)$ & $(4,98,-0,82)$ \\
$(6,3,7,4)$ & $(9,65,1,11)$ \\
$(8,0,7,1)$ & $(10,69,-0,27)$ \\
$(9,4,8,2)$ & $(12,47,-0,43)$ \\
\hline \hline
\end{tabular}

Tabela 3.2: Projeção das observações nos eixos alternativos.

Uma importante relação existe entre a decomposição em valores singulares da matriz $\hat{\mathbf{K}}$ e a decomposição espectral da matriz $\hat{\mathbf{K}}^{\prime} \hat{\mathbf{K}}$. Segundo Belsley (1991), o conjunto de autovalores e autovetores de $\hat{\mathbf{K}}^{\prime} \hat{\mathbf{K}}$ é tal que o conjunto de soluções do sistema dado por:

$$
\hat{\mathbf{K}}^{\prime} \hat{\mathbf{K}} \Theta=\Theta \Lambda .
$$

seja não trivial. Neste caso, $\Lambda$ é uma matriz diagonal $s \mathrm{x} s$ composta dos autovalores de $\hat{\mathbf{K}}^{\prime} \hat{\mathbf{K}}$, denominados $\check{\mu}_{j}$, e $\Theta^{\prime}$ é uma matriz $s \mathrm{x} p$ composta dos autovetores de $\hat{\mathbf{K}}^{\prime} \hat{\mathbf{K}}$ associados a $\check{\mu}_{j}$, denominados $\check{v}_{j}$ tal que $\boldsymbol{\Theta}^{\prime} \boldsymbol{\Theta}=\mathbf{I}_{\mathbf{s}}, j=0, \ldots, s-1$. 
Ao mesmo tempo, temos que, de (3.1), podemos escrever:

$$
\hat{\mathbf{K}}^{\prime} \hat{\mathbf{K}}=\mathbf{V D U}^{\prime} \mathbf{U D V}^{\prime}=\mathbf{V D}^{2} \mathbf{V}^{\prime}
$$

Se pós multiplicarmos ambos os lados da igualdade (3.3) por $\mathbf{V}$, teremos que:

$$
\hat{\mathbf{K}}^{\prime} \hat{\mathbf{K}} \mathbf{V}=\mathbf{V D}^{2}
$$

Comparando (3.4) com (3.2), temos que a matriz V obtida da decomposição em valores singulares de $\hat{\mathbf{K}}$ é igual à matriz $\Theta$ de autovetores de $\hat{\mathbf{K}}^{\prime} \hat{\mathbf{K}}$ e que a diagonal da matriz $\mathbf{D}$ é composta da raiz quadrada positiva dos elementos da diagonal da matriz $\Lambda$.

\subsubsection{Efeitos da multicolinearidade na estimação dos parâmetros}

Conforme foi visto, a estimativa da matriz de variância e covariância dos estimadores dos parâmetros do modelo de regressão logística é dada aproximadamente por $\widehat{\operatorname{Var}}(\hat{\beta})=$ $\left(\mathbf{X}^{\prime} \hat{\mathbf{W}} \mathbf{X}\right)^{-1}$. Como $\hat{\mathbf{W}}$ é uma matriz positiva definida, podemos reescrever $\mathbf{X}^{\prime} \hat{\mathbf{W}} \mathbf{X}$ como:

$$
\mathbf{X}^{\prime} \hat{\mathbf{W}} \mathbf{X}=\mathbf{X}^{\prime} \hat{\mathbf{W}}^{\mathbf{1} / \mathbf{2}} \hat{\mathbf{W}}^{\mathbf{1 / 2}} \mathbf{X}=\hat{\mathbf{K}}^{\prime} \hat{\mathbf{K}}
$$

em que $\hat{\mathbf{K}}=\hat{\mathbf{W}}^{\mathbf{1} / \mathbf{2}} \mathbf{X}$ e $\hat{\mathbf{W}}^{1 / 2}$ é a matriz $n \mathrm{x} n$ com elementos $\sqrt{\hat{\pi}_{i}\left(1-\hat{\pi}_{i}\right)}$ na diagonal principal, $i=1, \ldots, n$. Consequentemente, temos que:

$$
\widehat{\operatorname{Var}}(\hat{\beta})=\left(\hat{\mathbf{K}}^{\prime} \hat{\mathbf{K}}\right)^{-1}
$$

Além disso, sabe-se que $r\left(\hat{\mathbf{K}}^{\prime} \hat{\mathbf{K}}\right)=r(\mathbf{X})$, pois, segundo Seber e Lee (2003), se a matriz $\mathbf{B}$ é $n \mathrm{x} n$ positiva definida e a matriz $\mathbf{C}$ é uma matriz $n \times \mathrm{x} p$ qualquer, então $\mathrm{r}\left(\mathbf{C}^{\prime} \mathbf{B C}\right)=\mathrm{r}(\mathbf{C})$. Portanto, $\mathrm{r}\left(\hat{\mathbf{K}}^{\prime} \hat{\mathbf{K}}\right)=\mathrm{r}\left(\mathbf{X}^{\prime} \hat{\mathbf{W}} \mathbf{X}\right)=\mathrm{r}(\mathbf{X})=s<p<n$ na presença de multicolinearidade perfeita, o que nos leva a concluir que o fato da matriz $\mathbf{X}$ ser de posto não completo implica na matriz $\hat{\mathbf{K}}^{\prime} \hat{\mathbf{K}}$ ser mal condicionada.

Uma possível maneira de demonstrar os efeitos da multicolinearidade na estimação de $\beta$ quando $\mathbf{X}$ é de posto completo, é decompor a nova matriz de planejamento $\hat{\mathbf{K}}$ através da decomposição em valores singulares como em (3.1). Teremos, portanto, a seguinte estimativa assintótica da matriz de variância e covariância dos estimadores dos parâmetros do modelo de regressão logística:

$$
\widehat{\operatorname{Var}}(\hat{\beta})=\left(\hat{\mathbf{K}}^{\prime} \hat{\mathbf{K}}\right)^{-1}=\left(\mathbf{V D}^{\mathbf{2}} \mathbf{V}^{\prime}\right)^{-1}=\mathbf{V D}^{-\mathbf{2}} \mathbf{V}^{\prime}
$$

pois $\mathbf{V}$ é ortogonal, o que implica em $\mathbf{V}^{-1}=\mathbf{V}^{\prime}$. Portanto, para o k-ésimo componente de $\hat{\beta}$, teremos:

$$
\widehat{\operatorname{Var}}\left(\hat{\beta}_{k}\right)=\sum_{j=0}^{s-1} \frac{v_{k j}^{2}}{\mu_{j}^{2}},
$$


em que $v_{k j}$ é o k-ésimo elemento do j-ésimo autovetor e $\mu_{j}$ é o j-ésimo valor singular não nulo obtidos da decomposição em valores singulares de $\hat{\mathbf{K}}=\hat{\mathbf{W}}^{\mathbf{1 / 2}} \mathbf{X}$.

Podemos calcular também o erro quadrático médio de $\hat{\beta}$, ou seja, o valor esperado do quadrado da distância entre o verdadeiro vetor de parâmetros $\beta$ e o seu estimador de máxima verossimilhança $\hat{\beta}$. Denominando essa quantidade por $E Q M[\hat{\beta}]$ e fazendo a decomposição da matriz $\hat{\mathbf{K}}$ da mesma forma que em (3.1), temos, utilizando o fato de que o traço de $\hat{\mathbf{K}}^{\prime} \hat{\mathbf{K}}$ é a soma dos autovalores de $\hat{\mathbf{K}}^{\prime} \hat{\mathbf{K}}$, que:

$$
\begin{aligned}
E Q M[\hat{\beta}] & =E\left[(\beta-\hat{\beta})^{\prime}(\beta-\hat{\beta})\right] \\
& =\sum_{j=0}^{s-1} E\left[\left(\beta_{j}-\hat{\beta}_{j}\right)^{2}\right] \\
& =\sum_{j=0}^{s-1} \operatorname{Var}\left(\hat{\beta}_{j}\right) \\
& =\operatorname{tr}\left(\hat{\mathbf{K}}^{\prime} \hat{\mathbf{K}}\right)^{-1} \\
& =\sum_{j=0}^{s-1} \frac{1}{\mu_{j}} .
\end{aligned}
$$

Assumindo que existe uma relação linear exata entre as colunas da matriz $\hat{\mathbf{K}}$, então teremos que $\mathrm{r}(\hat{\mathbf{K}})=s<p$. Como, na decomposição em valores singulares de $\hat{\mathbf{K}}$, $\mathbf{U}$ e $\mathbf{V}$ são ortogonais e, consequentemente, de posto completo, teremos que $\mathrm{r}(\hat{\mathbf{K}})=\mathrm{r}(\mathbf{D})$. Assim, haverá tantos zeros na diagonal principal de $\mathbf{D}$ quanto for a quantidade de colunas dependentes de $\hat{\mathbf{K}}$, ou seja, devemos encontrar essa mesma quantidade de autovetores associados à $\hat{\mathbf{K}}$ iguais a zero. Como em aplicações práticas dificilmente encontramos uma dependência linear exata entre as covariáveis, teremos que essas relações serão expressas através de autovalores muito pequenos. Dessa forma, teremos, de (3.6) e (3.7), que a estimativa e a variância dos coeficientes estimados serão muito grandes e, portanto, distantes dos valores reais.

\subsubsection{Relação entre a multicolinearidade em modelos de regressão linear e logística}

Mostraremos a seguir que, na presença de multicolinearidade, tanto a estimação dos parâmetros quanto a da matriz de covariância dos estimadores dos parâmetros nos modelos de regressão logística ficam mais instáveis do que nos modelos de regressão linear.

Seja o modelo de regressão linear dado por:

$$
\mathbf{Y}^{*}=\beta_{0}^{*}+\beta_{1}^{*} \mathbf{x}_{1}^{*}+\ldots+\beta_{p-1}^{*} \mathbf{x}_{p-1}^{*}+\epsilon^{*}=\mathbf{X}^{*} \beta^{*}+\epsilon^{*}
$$

em que $\mathbf{Y}^{*}=\left(\mathbf{Y}_{1}^{*}, \mathbf{Y}_{2}^{*}, \ldots, \mathbf{Y}_{n}^{*}\right)^{\prime}$ é o vetor $n \mathrm{x} 1$ dos valores observados da variável resposta quantitativa de $n$ elementos amostrais independentes, $\mathbf{x}_{j}^{*}=\left(\mathbf{x}_{1 j}^{*}, \mathbf{x}_{2 j}^{*}, \ldots, \mathbf{x}_{n j}^{*}\right)^{\prime}$ é um vetor 
$n \mathrm{x} 1$ composto dos valores padronizados dos $n$ elementos amostrais da j-ésima covariável do modelo, $\mathbf{X}^{*}=\left(\mathbf{1}, \mathbf{x}_{1}^{*}, \mathbf{x}_{2}^{*}, \ldots, \mathbf{x}_{p-1}^{*}\right)$ é a matriz de planejamento do modelo de dimensão $n \times \mathrm{x} p$ tal que $\mathrm{r}\left(\mathbf{X}^{*}\right)=s^{*} \leq p<n, \mathbf{1}$ é um vetor $n \times 1$ de $1^{\prime}$ s, $\beta^{*}$ é um vetor $p \times 1$ de parâmetros desconhecidos e $\epsilon$ é um vetor $n \mathrm{x} 1$ dos erros aleatórios com a suposição de que $\epsilon^{*} \sim N_{n}\left(\mathbf{0}, \sigma^{2} \mathbf{I}\right)$, $i=1, \ldots, n, j=0, \ldots, p-1$.

Através da técnica de mínimos quadrados, presente em Gunst e Mason (1980), o estimador não viesado de mínima variância de $\beta^{*}$ do modelo de regressão linear é dado por:

$$
\hat{\beta}^{*}=\left[\left(\mathbf{X}^{*}\right)^{\prime} \mathbf{X}^{*}\right]^{-1}\left(\mathbf{X}^{*}\right)^{\prime} \mathbf{Y}^{*}
$$

Este estimador é não viesado, pois:

$$
E\left[\hat{\beta}^{*}\right]=\beta^{*}
$$

Já a matriz de variância e covariância de $\hat{\beta}^{*}$ é dada por:

$$
\operatorname{Var}\left[\hat{\beta}^{*}\right]=\sigma^{2}\left[\left(\mathbf{X}^{*}\right)^{\prime} \mathbf{X}^{*}\right]^{-1}
$$

Se houver multicolinearidade entre as covariáveis do modelo de regressão linear, cada componente presente em $\hat{\beta}^{*}$, assim como cada uma das suas variâncias e covariâncias, ficarão superestimadas, pois, fazendo a decomposição em valores singulares da matriz $\mathbf{X}^{*}$, temos que:

$$
\left[\left(\mathbf{X}^{*}\right)^{\prime} \mathbf{X}^{*}\right]_{j j}=\sum_{k=0}^{s^{*}-1} \frac{v_{k j}^{* 2}}{\mu_{k}^{* 2}},
$$

em que $\left[\left(\mathbf{X}^{*}\right)^{\prime} \mathbf{X}^{*}\right]_{j j}$ é o j-ésimo elemento da diagonal principal de $\left[\left(\mathbf{X}^{*}\right)^{\prime} \mathbf{X}^{*}\right]^{-1}$ e $v_{k j}^{*}$ e $\mu_{k}^{*}$ são, respectivamente, o k-ésimo elemento do j-ésimo autovetor e o k-ésimo autovalor obtidos da decomposição em valores singulares de $\mathbf{X}^{*}$. Como a variância e a covariância das componentes de $\hat{\beta}^{*}$, bem como as suas estimativas, são funções de $\left[\left(\mathbf{X}^{*}\right)^{\prime} \mathbf{X}^{*}\right]^{-1}$ e, se houver multicolinearidade, pelo menos um valor de $\mu_{k}^{*}$ será pequeno, então todas essas medidas estarão superestimadas. Além disso, o erro quadrático médio de $\hat{\beta}^{*}$, dado por $E Q M\left[\hat{\beta}^{*}\right]$, também ficará superestimado, pois, segundo Hoerl e Kennard (1970): 


$$
\begin{aligned}
E Q M\left[\hat{\beta}^{*}\right] & =E\left[\left(\beta^{*}-\hat{\beta}^{*}\right)^{\prime}\left(\beta^{*}-\hat{\beta}^{*}\right)\right] \\
& =\sum_{j=0}^{s-1} E\left[\left(\beta_{j}^{*}-\hat{\beta}_{j}^{*}\right)^{2}\right] \\
& =\sum_{j=0}^{s-1} \operatorname{Var}\left(\hat{\beta}_{j}^{*}\right) \\
& =\sigma^{2} \operatorname{tr}\left(\left(\mathbf{X}^{*}\right)^{\prime} \mathbf{X}^{*}\right)^{-1} \\
& =\sigma^{2} \sum_{j=0}^{s^{*}-1} \frac{1}{\mu_{j}^{* 2}} .
\end{aligned}
$$

Sem perda de generalidade, assuma que existe somente uma relação linear aproximada entre as covariáveis, portanto, somente $\mu_{s-1}^{*}$ será próximo de zero. De acordo com Schaefer (1986), $\mu_{s-1}^{2} \leq(1 / 4) \mu_{s-1}^{* 2}$, ou seja, o menor autovalor obtido da decomposição em valores singulares da matriz de planejamento do modelo de regressão logística, dada por $\hat{\mathbf{K}}$, é menor do que o menor autovalor da matriz de planejamento do modelo de regressão linear, dada por $\mathrm{X}^{*}$. Note ainda que:

$$
\widehat{\operatorname{Var}}\left[\hat{\beta}_{j}\right]=\left(\hat{\mathbf{K}}^{\prime} \hat{\mathbf{K}}\right)_{j j}=\sum_{k=0}^{s-1} \frac{v_{k j}^{2}}{\mu_{k}^{2}} \geq \frac{v_{s-1 j}^{2}}{\mu_{s-1}^{2}} \geq 4 \frac{v_{s-1 j}^{* 2}}{\mu_{s-1}^{* 2}},
$$

em que $\left(\hat{\mathbf{K}}^{\prime} \hat{\mathbf{K}}\right)_{j j}$ é o $(j, j)$-ésimo elemento de $\left(\hat{\mathbf{K}}^{\prime} \hat{\mathbf{K}}\right)^{-1}$. Isso significa que a existência de multicolinearidade no modelo de regressão linear implica na existência de multicolinearidade ainda mais severa no modelo de regressão logística.

Portanto, na presença de multicolinearidade, as estimativas de $\beta^{*}$, as estimativas das variâncias dos estimadores de seus componentes e de seu erro quadrático médio estarão distantes dos valores reais.

\subsubsection{Efeitos da multicolinearidade na inferência sobre os parâme- tros}

Conforme foi visto no Capítulo 2, uma forma de testar se um parâmetro é diferente de zero (individualmente) é através da estatística de teste de Wald. Para testar a hipótese nula de que $\beta_{k}$ é igual a zero, calculamos a estatística dada por (2.12). De acordo com (3.6), podemos reescrever (2.12) como:

$$
\text { Wald }_{k}=\frac{\hat{\beta}_{k}}{\sqrt{\sum_{j=0}^{p-1} \frac{v_{k j}^{2}}{\mu_{j}^{2}}}} .
$$

Na presença de multicolinearidade, teremos pelo menos um $\mu_{j}$ muito pequeno, fazendo com que a correspondente estatística de Wald fique próxima de zero, mesmo que $\beta_{k}$ não seja 
nulo. Por outro lado, mesmo que $\mu_{j}$ não seja tão pequeno, $\hat{\beta}_{k}$ pode estar inflacionado devido à multicolinearidade. Dessa forma, a inferência com base nesta estatística fica seriamente comprometida, podendo ficar muito maior ou muito menor do que o seu verdadeiro valor, resultando em uma menor chance de a hipótese nula ser não rejeitada, no primeiro caso, ou resultando em uma maior chance de a hipótese nula ser rejeitada, no último caso.

Foi visto ainda que podemos testar a significância conjunta das covariáveis do modelo através do teste de razão de verossimilhanças e este teste é a base de métodos de seleção de covariáveis, como o stepwise. A importância de cada covariável é medida através da diferença entre o logaritmo da função de verossimilhança do modelo com essa variável e o logaritmo da função de verossimilhança do modelo sem essa variável. Portanto, para testar se o parâmetro relacionado à covariável $\mathbf{x}_{\mathbf{k}}, k=1, \ldots, p$-1, é estatisticamente significante, é necessário modificar a estatística (2.13) para:

$$
G^{(k)}=-2 \ln \left[\frac{\prod_{i=1}^{n}\left(\hat{\pi}_{i}^{(+k)}\right)^{Y_{i}}\left(1-\hat{\pi}_{i}^{(+k)}\right)^{\left(1-Y_{i}\right)}}{\prod_{i=1}^{n}\left(\hat{\pi}_{i}^{(-k)}\right)^{Y_{i}}\left(1-\hat{\pi}_{i}^{(-k)}\right)^{\left(1-Y_{i}\right)}}\right],
$$

em que $\hat{\pi}_{i}^{(+k)}$ é a probabilidade de sucesso do i-ésimo indivíduo estimada pelo modelo com a k-ésima variável e $\hat{\pi}_{i}^{(-k)}$ é a probabilidade de sucesso do i-ésimo indivíduo estimada pelo modelo retirada a k-ésima variável. Conforme comentado anteriormente, na presença de multicolinearidade $\beta$ pode fica superestimado. Consequentemente, $\hat{\pi}_{i}{ }^{(+k)}$ e $\hat{\pi}_{i}{ }^{(-k)}$ ficarão distantes dos valores reais, fazendo com que a estatística dada por (3.10) fique distorcida. Dessa forma, a covariável pode ser retirada ou incluída no modelo sem ser necessariamente significante.

\subsection{Diagnóstico da multicolinearidade}

Segundo Neter et al. (2005), as seguintes técnicas informais de diagnóstico podem ser utilizados para avaliar indícios da presença de multicolinearidade:

- duas ou mais covariáveis independentes são correlacionadas;

- o sinal e $\backslash$ ou a ordem de grandeza das estimativas dos coeficientes são diferentes do que o esperado;

- ocorrem grandes mudanças nas estimativas dos coeficientes quando variáveis são adicionadas ou excluídas do modelo;

- resultados dos testes individuais para os coeficientes do modelo de importantes variáveis preditoras são não significantes.

Não podemos, porém, nos basear somente nesses indícios, pois eles não são condições necessárias e suficientes para a multicolinearidade. O correto diagnóstico deve ter ligação direta com a sua definição, ou seja, com a dependência linear exata ou aproximada entre 
as colunas da matriz de planejamento. Por essa razão, vamos definir nesta sessão diferentes formas de identificação da multicolinearidade: Coeficiente de Determinação $\left(R^{2}\right)$, Índice e Número de Condição ( $\eta$ e $\eta^{*}$, respectivamente) e Decomposição da Variância $(\varphi)$.

\subsubsection{Coeficiente de determinação}

Seja o modelo de regressão linear dado por (3.8). Para determinarmos o nível da relação entre duas ou mais covariáveis, podemos calcular uma medida de associação entre elas. Schaefer et al. (1984), por exemplo, utiliza o coeficiente de determinação da regressão linear da j-ésima covariável em relação às demais, que será denominado $R_{j}^{2}$. O coeficiente $R_{j}^{2}$ é tal que $0 \leq R_{j}^{2} \leq 1$ e altos valores de $R_{j}^{2}$ são indicativos de multicolinearidade. Em Schaefer et al. (1984), o autor cita que em Schaefer (1979) é mostrado que a caracterização da multicolinearidade em modelos de regressão logística através do $R_{j}^{2}$ é equivalente à caracterização através dos autovalores de $\hat{\mathbf{K}}^{\prime} \hat{\mathbf{K}}$ pequenos.

A ideia para a utilização deste coeficiente é que, se houver alguma relação linear entre quaisquer covariáveis, então quando ajustarmos um modelo de regressão linear para uma covariável em função das demais, o valor observado da covariável em questão e o seu valor predito através das demais covariáveis serão próximos. Vale ressaltar que todas as covariáveis deveriam ser quantitativas para que o modelo de regressão linear pudesse ser utilizado. Porém, como o intuito aqui é simplesmente achar um índice para identificar a multicolinearidade, vamos considerar também as covariáveis qualitativas com suas categorias codificadas em variáveis indicadoras.

Podemos escrever, portanto, a covariável $\mathbf{x}_{j}^{*}$ como função das demais covariáveis, obtendo o modelo de regressão:

$$
\begin{aligned}
\mathbf{x}_{j}^{*} & =\beta_{0}^{*}+\beta_{1}^{*} \mathbf{x}_{1}^{*}+\ldots+\beta_{j-1}^{*} \mathbf{x}_{j-1}^{*}+\beta_{j+1}^{*} \mathbf{x}_{j+1}^{*}+\ldots+\beta_{p-1}^{*} \mathbf{x}_{p-1}^{*}+\epsilon_{(j)}^{*} \\
& =\mathbf{X}_{(j)} \beta_{(j)}+\epsilon_{(j)}^{*}
\end{aligned}
$$

em que $\mathbf{X}_{(j)}=\left(1, \mathbf{x}_{1}^{*}, \ldots, \mathbf{x}_{j-1}^{*}, \mathbf{x}_{j+1}^{*}, \ldots, \mathbf{x}_{p-1}^{*}\right)$ é uma matriz $n x p$-1 que não contém a j-ésima covariável, $\beta_{(j)}$ é um vetor $p$-1x1 de todos os parâmetros desconhecidos exceto pelo j-ésimo e $\epsilon_{(j)}^{*}$ é um vetor $n \times 1$ composto dos erros aleatórios, $j=1, \ldots, p-1$.

Dessa forma, segundo Gunst e Mason (1980), temos que:

$$
\mathbf{x}_{j}^{*}=\hat{\mathbf{x}}_{j}^{*}+\mathbf{r}_{i}, \quad j=1, \ldots, p-1,
$$

em que $\hat{\mathbf{x}}_{j}^{*}=\mathbf{X}_{(j)} \hat{\beta}_{(j)}$ e $\mathbf{r}_{i}$ é o vetor dos resíduos produzidos quando aproximamos $\mathbf{x}_{j}^{*}$ por $\hat{\mathbf{x}}_{j}^{*}$. Se $\mathbf{r}_{i}=0$, então temos que $\mathbf{x}_{j}^{*}=\hat{\mathbf{x}}_{j}^{*}$, ou seja, se construirmos o gráfico de $\mathbf{x}_{j}^{*}$ contra $\hat{\mathbf{x}}_{j}^{*}$ todos os pontos estarão sobre uma reta. Além disso, teremos que $R_{j}$, ou seja, o coeficiente de correlação de Pearson, assumirá o seu maior valor, \pm 1 . Em contrapartida, quando os pontos ficarem praticamente aleatórios ou seguirem um padrão não linear no gráfico de $\mathbf{x}_{j}^{*}$ contra 
$\hat{\mathbf{x}}_{j}^{*}$ então obteremos o menor valor absoluto para $R_{j}$, ou seja, $R_{j}=0$.

O coeficiente de correlação de Pearson é dado por:

$$
R_{j}=\frac{\sum_{i=1}^{n}\left(x_{i j}^{*}-\bar{x}_{j}^{*}\right)\left(\hat{x}_{i j}^{*}-M_{j}\right)}{\left[\sum_{i=1}^{n}\left(x_{i j}^{*}-\bar{x}_{j}^{*}\right)^{2} \sum_{i=1}^{n}\left(\hat{x}_{i j}^{*}-M_{j}\right)^{2}\right]^{1 / 2}}, \quad j=1, \ldots, p-1,
$$

em que $M_{j}$ é o valor médio predito da j-ésima covariável. Como não nos interessa se a associação é positiva ou negativa, mas sim, se ela existe ou não, então podemos usar o quadrado do coeficiente de correlação de Pearson, ou seja, $R_{j}^{2}$. Se os resíduos são insignificantes, então $R_{j}^{2}$ será próximo de 1 ; caso contrário, $\mathbf{x}_{j}^{*}$ e $\hat{\mathbf{x}}_{j}^{*}$ não serão correlacionadas e, portanto, $R_{j}^{2}$ será próximo de 0.

O quadrado do valor do coeficiente de correlação de Pearson é chamado de Coeficiente de Determinação. Além de calcularmos o coeficiente de determinação como o quadrado do valor dado por (3.11), Gunst e Mason (1980) demonstra que podemos escrevê-lo como sendo:

$$
\begin{aligned}
R_{j}^{2} & =\frac{\sum_{i=1}^{n}\left(\hat{x}_{i j}^{*}-M_{j}\right)^{2}}{\sum_{i=1}^{n}\left(\hat{x}_{i j}^{*}-M_{j}\right)^{2}+\sum_{i=1}^{n}\left(x_{i j}^{*}-\hat{x}_{i j}^{*}\right)^{2}} \\
& =\frac{\sum_{i=1}^{n}\left(\hat{x}_{i j}^{*}-M_{j}\right)^{2}}{\sum_{i=1}^{n}\left(x_{i j}^{*}-M_{j}\right)^{2}} .
\end{aligned}
$$

O numerador de (3.12) é conhecido como Soma de Quadrados da Regressão e mede a variabilidade dos valores preditos em relação à média de $\mathbf{x}_{j}^{*}$ observada. Já a primeira parte do numerador na primeira igualdade é conhecido como Soma de Quadrados dos Resíduos e mede a variabilidade de $\mathbf{x}_{j}^{*}$ que não pode ser explicada pelo modelo predito. O denominador da segunda igualdade, por sua vez, é conhecido como Soma de Quadrados Total Ajustada e mede a variabilidade total da variável resposta.

O coeficiente $R_{j}^{2}$ pode, portanto, ser interpretado como a proporção da variabilidade de $\mathbf{x}_{j}^{*}$ atribuída ao modelo estimado, ou seja, às demais covariáveis. Dessa forma, se houver multicolinearidade entre as covariáveis, teremos que uma grande proporção da variabilidade de $\mathbf{x}_{j}^{*}$ será explicada pelas demais covariáveis, resultando em um valor de $R_{j}^{2}$ muito próximo de 1 .

De acordo com Montgomery et al. (2001), não se pode determinar um valor ótimo para $R_{j}^{2}$. Porém, este coeficiente é função do Fator de Inflação da Variância (VIF, Variance Inflation Factor) da j-ésima covariável, dado por:

$$
V I F_{j}=\frac{1}{1-R_{j}^{2}}, \quad j=1, \ldots, p-1
$$

A medida VIF é um importante indicador de multicolinearidade na regressão linear, pois a variância dos estimadores dos parâmetros do modelo pode ser reescrita como:

$$
\operatorname{Var}\left[\hat{\beta}_{j}^{*}\right]=\frac{\sigma^{2}}{1-R_{j}^{2}}, \quad j=1, \ldots, p-1
$$


Na presença de multicolinearidade, o valor de $R_{j}^{2}$ será muito próximo de 1 , fazendo com que a variância de $\hat{\beta}_{j}^{*}$ fique inflacionada. Segundo Montgomery et al. (2001), valores de VIF maiores do que 5 indicam multicolinearidade moderada e valores de VIF maiores do que 10 implicam em multicolinearidade severa. Na Tabela 3.3 encontra-se um resumo desta classificação.

\begin{tabular}{ccc}
\hline \hline$V I F_{j}$ & $R_{j}^{2}$ & Multicolinearidade \\
\hline$<5$ & $<90 \%$ & Fraca \\
{$[5 ; 10[$} & {$[90 \% ; 95 \%$} & Moderada \\
$\geq 10$ & $\geq 95 \%$ & Severa \\
\hline \hline
\end{tabular}

Tabela 3.3: Classificação da multicolinearidade.

Portanto, devido à sua fácil interpretação e por ser uma função do coeficiente de correlação de Pearson, $R_{j}^{2}$ é uma medida muito importante para avaliar a adequação da predição do modelo e, consequentemente, uma medida muito importante para avaliar o nível de multicolinearidade entre as covariáveis. Em contrapartida, segundo Belsley (1991), este coeficiente também apresenta algumas deficiências. A primeira e mais importante é que o coeficiente de determinação não é capaz de diagnosticar a quantidade de dependências aproximadas presentes nas colunas da matriz de planejamento. Se, por exemplo, existem duas fortes dependências entre as colunas de $\hat{\mathbf{K}}$, digamos que a primeira envolve $\hat{\mathbf{K}}_{1}, \hat{\mathbf{K}}_{2}, \hat{\mathbf{K}}_{4}$ e $\hat{\mathbf{K}}_{5}$ e a segunda envolve $\hat{\mathbf{K}}_{4}$ e $\hat{\mathbf{K}}_{5}$, os quatro coeficientes de determinação serão próximos de 1 . Isso também será verdade se somente a primeira relação existir. A segunda deficiência deste coeficiente é que, apesar de serem propostos alguns limites para indicar fraca, moderada e forte dependência entre as colunas de $\hat{\mathbf{K}}$, não há na literatura limites para os valores de $R_{j}^{2}$.

\subsection{2 Índice e número de condição}

Até o presente momento comentamos que se existir uma relação linear aproximada entre as covariáveis do modelo, então pelo menos um autovalor obtido da decomposição em valores singulares da matriz de planejamento do modelo será pequeno. Não foi discutido, porém, quão pequeno ele deve ser.

De acordo com (3.1), podemos obter os $s$ valores singulares da matriz de planejamento do modelo. Se dividirmos o maior autovalor, $\mu_{0}$, por cada um dos demais, chegamos ao j-ésimo índice de condição:

$$
\eta_{j}=\frac{\mu_{0}}{\mu_{j}}, j=0, \ldots, s-1 .
$$

Segundo Belsley et al. (2004), haverá tantas dependências aproximadas entre as colunas da matriz $\hat{\mathbf{K}}$ quantas forem a quantidade de índices de condição grandes. Além disso, índices de condição entre 5 e 10 implicam em fraca dependência, enquanto dependências moderadas a fortes estão associadas a índices de condição entre 30 e 100. 
O índice de condição de $\hat{\mathbf{K}}$ é definido como o máximo valor de $\eta_{j}, \mathrm{j}=0, \ldots, \mathrm{s}-1$, ou seja, será dado por:

$$
\eta=\frac{\mu_{0}}{\mu_{s-1}}
$$

Ainda, de acordo com Montgomery et al. (2001), outra forma de se definir o nível de associação entre as colunas da matriz é através do número de condição de $\hat{\mathbf{K}}^{\prime} \hat{\mathbf{K}}$. Conforme foi visto, os autovalores da matriz $\hat{\mathbf{K}}^{\prime} \hat{\mathbf{K}}$ são iguais ao quadrado dos valores singulares da matriz $\hat{\mathbf{K}}$. Portanto, podemos definir o j-ésimo número de condição de $\hat{\mathbf{K}}^{\prime} \hat{\mathbf{K}}$ :

$$
\eta_{j}^{*}=\frac{\mu_{0}^{2}}{\mu_{j}^{2}}, j=1, \ldots, s .
$$

O número de condição de $\hat{\mathbf{K}}^{\prime} \hat{\mathbf{K}}$ será o maior deles, ou seja, será dado por:

$$
\eta^{*}=\frac{\mu_{0}^{2}}{\mu_{s-1}^{2}} .
$$

Segundo Montgomery et al. (2001), quando o número de condição é menor do que 100, as covariáveis não sofrem de multicolinearidade. Quando o número de condição estiver entre 100 e 1000 teremos forte multicolinearidade e se esse índice for maior do que 1000, teremos multicolinearidade severa. De acordo com Belsley (1991), esse coeficiente mede a dificuldade para se inverter a matriz $\hat{\mathbf{K}}^{\prime} \hat{\mathbf{K}}$.

Ao contrário do coeficiente de determinação, de acordo com Belsley (1991), tanto o índice como o número de condição são medidas que apresentam limites para indicar quando a dependência entre as colunas da matriz de planejamento é forte e, além disso, quantas dependências existem, baseada na quantidade de $\eta_{j}$ 's ou $\eta_{j}^{*}$ 's grandes.

\subsubsection{Decomposição da variância}

Como já foi mostrado em (3.6), a estimativa da variância estimada de cada um dos estimadores das componentes de $\beta$ pode ser decomposta em uma soma de termos associados aos autovalores obtidos da decomposição em valores singulares da matriz de planejamento do modelo de regressão logística. Essa decomposição fornece o elo entre a análise numérica da matriz de planejamento $\hat{\mathbf{K}}$, através da sua decomposição em valores singulares, e a qualidade da análise de regressão logística usando $\hat{\mathbf{K}}$ como a matriz de planejamento, através da matriz de variância e covariância de $\hat{\beta}$.

De (3.6), temos que a estimativa da variância de cada $\hat{\beta}_{j}$ pode ser escrita como uma soma de componentes, cada um associado a somente um dos $s$ valores singulares $\mu_{j}$. Como $\mu_{j}$ aparece no denominador, todos os componentes associados à multicolinearidade, ou seja, com $\mu_{j}$ pequeno, serão grandes em relação aos demais. De acordo com Belsley (1991), grandes proporções das variâncias de dois ou mais coeficientes concentradas em componentes associados aos mesmos pequenos valores singulares proporcionam evidências que as colunas de $\hat{\mathbf{K}}$ 
associados aos coeficientes envolvidos nas multicolinearidades correspondem aos pequenos valores singulares. Seja:

$$
\phi_{k j}=\frac{v_{k j}^{2}}{\mu_{j}^{2}}, \quad k, j=0, \ldots, s-1,
$$

a proporção da variância do k-ésimo coeficiente da regressão associado ao j-ésimo componente da sua decomposição e seja:

$$
\phi_{k}=\operatorname{Var}\left(\hat{\beta}_{k}\right)=\sum_{j=0}^{s-1} \phi_{k j}, \quad k=0, \ldots, s-1 .
$$

Então, a proporção da decomposição da variância do k-ésimo coeficiente da regressão associado ao j-ésimo componente da sua decomposição é dada por:

$$
\omega_{k j}=\frac{\phi_{k j}}{\phi_{k}}, \quad k, j=0, \ldots, s-1 .
$$

De acordo com Belsley et al. (2004), grandes proporções da decomposição da variância $(>0,5)$ para duas ou mais variâncias estimadas dos coeficientes da regressão logística correspondendo aos mesmos pequenos valores singulares associados aos grandes índices de condição levam à identificação das covariáveis envolvidas na multicolinearidade.

A decomposição da variância é melhor sumarizada quando apresentada em uma tabela como a Tabela 3.4. Nela, cada linha é associada a um autovalor, ou, equivalentemente, a um índice de condição, e, naturalmente, cada coluna deve somar 1.

\begin{tabular}{lcclc}
\hline \hline Índice de & Proporção de & Proporção de & $\ldots$ & Proporção de \\
Condição & $\operatorname{Var}\left[\hat{\beta}_{0}\right]$ & $\operatorname{Var}\left[\hat{\beta}_{1}\right]$ & $\ldots$ & $\operatorname{Var}\left[\hat{\beta}_{s-1}\right]$ \\
\hline$\eta_{0}$ & $\omega_{00}$ & $\omega_{01}$ & $\ldots$ & $\omega_{0 s-1}$ \\
$\eta_{1}$ & $\omega_{10}$ & $\omega_{11}$ & $\ldots$ & $\omega_{1 s-1}$ \\
$\vdots$ & $\vdots$ & $\vdots$ & & $\vdots$ \\
$\eta_{s-1}$ & $\omega_{s-10}$ & $\omega_{s-11}$ & $\ldots$ & $\omega_{s-1 s-1}$ \\
\hline \hline
\end{tabular}

Tabela 3.4: Matriz com a Proporção da Decomposição da Variância.

\subsection{Considerações finais do capítulo}

Neste capítulo apresentamos os efeitos da multicolinearidade na estimação e na inferência dos parâmetros do modelo de regressão logística, bem como algumas técnicas para quantificar a multicolinearidade e identificar as covariáveis que estão envolvidas neste problema. Mostramos também que é mais grave a multicolinearidade estar presente na matriz de planejamento de modelos de regressão logística do que na de modelos de regressão linear. Vale ressaltar, porém, que, de acordo com Lesaffre e Marx (1993), a matriz de covariância 
assintótica dos estimadores de $\beta$ é $\left(\mathbf{X}^{\prime} \mathbf{W X}\right)^{-1}$. De modo geral, $\mathbf{W}$ é positiva definida, mas, como na prática $\mathbf{W}$ é estimada por $\hat{\mathbf{W}}$, se algum elemento da diagonal principal de $\hat{\mathbf{W}}$ for zero, $\mathbf{X}^{\prime} \hat{\mathbf{W X}}$ será singular. Os autores apresentam ainda um exemplo em que o número de condição de $\mathbf{X}^{\prime} \mathbf{X}$ é 18,6, mas o da matriz $\mathbf{X}^{\prime} \hat{\mathbf{W} X}$ é 100,75. Desta forma, pode haver multicolinearidade no modelo de regressão logística mesmo que não exista multicolinearidade entre as covariáveis e o analista deve estar bastante atento a esse fato.

Com o objetivo de minimizar os efeitos da multicolinearidade, a seguir, serão discutidos dois estimadores alternativos ao de máxima verossimilhança: Estimador em Cristas e Estimador em Componentes Principais. 


\section{Capítulo 4}

\section{Estimadores Alternativos para os Parâmetros do Modelo de Regressão Logística}

De acordo com Bolfarine e Sandoval (2001), estimadores de máxima verossimilhança apresentam propriedades muito importantes, como a de serem, assintoticamente, estimadores não viesados e de terem mínima variância (dentre os estimadores não viesados). Porém, como visto no Capítulo 3, a multicolinearidade pode afetar a eficiência desses estimadores, aumentando exacerbadamente as estimativas pontuais e as variâncias dos estimadores. O objetivo deste capítulo é propor duas técnicas que introduzem um pequeno viés nos estimadores de máxima verossimilhança nos modelos de regressão logística, com o intuito de diminuir drasticamente suas variâncias. São elas: Regressão em Cristas e Regressão em Componentes Principais.

\subsection{Regressão em Cristas}

A regressão em cristas foi sugerida pela primeira vez por A. E. Hoerl no artigo Hoerl (1962), com o intuito de controlar a inflação da variância e a instabilidade associadas aos estimadores de mínimos quadrados da regressão linear. Portanto, vamos, primeiramente, mostrar o desenvolvimento deste estimador na regressão linear para, depois, desenvolvê-lo na regressão logística. Para finalizar, apresentaremos algumas formas de selecionar o melhor parâmetro em cristas.

\subsubsection{Regressão Linear em Cristas}

Seja o modelo de regressão linear dado por (3.8). Hoerl e Kennard (1970) notaram que se

$\beta^{*}$ fosse estimado de tal forma que a soma de quadrados dos resíduos, $\phi_{m i n}^{*}$, fosse acrescida de um pequeno valor, isso seria suficiente para diminuir as suas estimativas pontuais. Assim, denotando por $\beta_{C}^{*}\left(\lambda^{*}\right)$ esse novo estimador que será definido posteriormente, temos que: 


$$
\begin{aligned}
\phi^{*} & =\left[\mathbf{Y}^{*}-\mathbf{X}^{*} \beta_{C}^{*}\left(\lambda^{*}\right)\right]^{\prime}\left[Y^{*}-\mathbf{X}^{*} \beta_{C}^{*}\left(\lambda^{*}\right)\right] \\
& =\left[\mathbf{Y}^{*}-\mathbf{X}^{*} \hat{\beta}^{*}\right]^{\prime}\left[Y^{*}-\mathbf{X}^{*} \hat{\beta}^{*}\right]+\left[\beta_{C}^{*}\left(\lambda^{*}\right)-\hat{\beta}^{*}\right]^{\prime}\left(\mathbf{X}^{*}\right)^{\prime} \mathbf{X}^{*}\left[\beta_{C}^{*}\left(\lambda^{*}\right)-\hat{\beta}^{*}\right] \\
& =\phi_{\min }^{*}+\phi^{*}\left(\beta_{C}^{*}\left(\lambda^{*}\right)\right),
\end{aligned}
$$

em que $\phi_{m i n}^{*}$ é a soma de quadrados dos resíduos quando o estimador de $\beta^{*}$ é o estimador de mínimos quadrados $\hat{\beta}^{*}$ e $\phi^{*}\left(\beta_{C}^{*}\left(\lambda^{*}\right)\right)$ é a forma quadrática em $\left[\beta_{C}^{*}\left(\lambda^{*}\right)-\hat{\beta}^{*}\right]$. Portanto, para encontrar o melhor valor de $\beta_{C}^{*}\left(\lambda^{*}\right)$ teremos que minimizar $\left(\beta_{C}^{*}\left(\lambda^{*}\right)\right)^{\prime} \beta_{C}^{*}\left(\lambda^{*}\right)$ fixado $\phi^{*}\left(\beta_{C}^{*}\left(\lambda^{*}\right)\right)$. Utilizando o Método de Lagrange, será necessário minimizar:

$$
F^{*}=\left[\beta_{C}^{*}\left(\lambda^{*}\right)\right]^{\prime} \beta_{C}^{*}\left(\lambda^{*}\right)+\frac{1}{\lambda^{*}}\left\{\left[\beta_{C}^{*}\left(\lambda^{*}\right)-\hat{\beta}^{*}\right]^{\prime}\left(X^{*}\right)^{\prime} X^{*}\left[\beta_{C}^{*}\left(\lambda^{*}\right)-\hat{\beta}^{*}\right]-\phi^{*}\left(\beta_{C}^{*}\left(\lambda^{*}\right)\right)\right\}
$$

em que $\frac{1}{\lambda^{*}}$ é o multiplicador de Lagrange. Derivando (4.1) em relação à $\beta_{C}^{*}\left(\lambda^{*}\right)$ e igualando à zero, teremos:

$$
\left.\frac{\partial F^{*}}{\partial \beta_{C}^{*}\left(\lambda^{*}\right)}=2 \beta_{C}^{*}\left(\lambda^{*}\right)+\frac{1}{\lambda^{*}}\left[2\left(X^{*}\right)^{\prime} X^{*} \beta_{C}^{*}\left(\lambda^{*}\right)-2\left(X^{*}\right)^{\prime} X^{*} \hat{\beta}^{*}\right)\right]=0 .
$$

Por fim, a equação (4.2) se reduz ao estimador em cristas, que é dado por:

$$
\begin{aligned}
\hat{\beta}_{C}^{*}\left(\lambda^{*}\right) & =\left[\left(\mathbf{X}^{*}\right)^{\prime} \mathbf{X}^{*}+\lambda^{*} \mathbf{I}\right]^{-1}\left(\mathbf{X}^{*}\right)^{\prime} \mathbf{Y}^{*} \\
& =\left\{\mathbf{I}_{p}+\lambda^{*}\left[\left(X^{*}\right)^{\prime} X^{*}\right]^{-1}\right\}^{-1} \hat{\beta}^{*} \\
& =\mathbf{Z} \hat{\beta}^{*}
\end{aligned}
$$

em que $\lambda^{*}$ é o parâmetro em cristas tal que $\lambda^{*} \geq 0$ e $\mathbf{Z}=\left\{\mathbf{I}_{p}+\lambda^{*}\left[\left(X^{*}\right)^{\prime} X^{*}\right]^{-1}\right\}^{-1}$.

Analisando o erro quadrático médio do estimador dado por (4.3), $E Q M\left(\hat{\beta}_{C}^{*}\left(\lambda^{*}\right)\right)$, e utilizando (4.4), Hoerl e Kennard (1970) prova que:

$$
\begin{aligned}
\operatorname{EQM}\left(\hat{\beta}_{C}^{*}\left(\lambda^{*}\right)\right) & =E\left[\left(\hat{\beta}_{C}^{*}\left(\lambda^{*}\right)-\beta^{*}\right)^{\prime}\left(\hat{\beta}_{C}^{*}\left(\lambda^{*}\right)-\beta^{*}\right)\right] \\
& =\sigma^{2} \sum_{j=0}^{s^{*}-1} \frac{\mu_{j}^{* 2}}{\left(\mu_{j}^{* 2}+\lambda^{*}\right)^{2}}+\lambda^{* 2}\left(\beta^{*}\right)^{\prime}\left[\left(\mathbf{X}^{*}\right)^{\prime} \mathbf{X}^{*}+\lambda^{*} \mathbf{I}\right]^{-2} \beta^{*} \\
& =\gamma_{1}\left(\lambda^{*}\right)+\gamma_{2}\left(\lambda^{*}\right),
\end{aligned}
$$

em que $\gamma_{1}\left(\lambda^{*}\right)=\sigma^{2} \sum_{j=0}^{s^{*}-1} \frac{\mu_{j}^{* 2}}{\left(\mu_{j}^{* 2}+\lambda^{*}\right)^{2}}$ e $\gamma_{2}\left(\lambda^{*}\right)=\lambda^{* 2}\left(\beta^{*}\right)^{\prime}\left[\left(\mathbf{X}^{*}\right)^{\prime} \mathbf{X}^{*}+\lambda^{*} \mathbf{I}\right]^{-2} \beta^{*}$. Portanto, o erro quadrático médio de $\hat{\beta}_{C}^{*}\left(\lambda^{*}\right)$ é composto da soma das variâncias dos estimadores dos parâmetros, dado pelo primeiro elemento $\gamma_{1}\left(\lambda^{*}\right)$, mais o quadrado do erro cometido quando 
se utiliza $\hat{\beta}_{C}^{*}\left(\lambda^{*}\right)$ ao invés de $\hat{\beta}^{*}$, dado pelo segundo elemento $\gamma_{2}\left(\lambda^{*}\right)$. Além disso, escrevendo o estimador em cristas na regressão linear como $\hat{\beta}_{C}^{*}\left(\lambda^{*}\right)=\mathbf{Z} \hat{\beta}^{*}=\mathbf{Z}\left[\left(\mathbf{X}^{*}\right)^{\prime} \mathbf{X}^{*}\right]^{-1}\left(\mathbf{X}^{*}\right)^{\prime} \mathbf{Y}$, sua matriz de covariância pode ser descrita como:

$$
\begin{aligned}
\operatorname{Var}\left[\hat{\beta}_{C}^{*}\left(\lambda^{*}\right)\right] & =\mathbf{Z}\left[\left(\mathbf{X}^{*}\right)^{\prime} \mathbf{X}^{*}\right]^{-1}\left(\mathbf{X}^{*}\right)^{\prime} \operatorname{Var}[\mathbf{Y}] \mathbf{X}^{*}\left[\left(\mathbf{X}^{*}\right)^{\prime} \mathbf{X}^{*}\right]^{-1} \mathbf{Z}^{\prime} \\
& =\sigma^{2} \mathbf{Z}\left[\left(\mathbf{X}^{*}\right)^{\prime} \mathbf{X}^{*}\right]^{-1} \mathbf{Z}^{\prime}
\end{aligned}
$$

Através da Figura 4.1, retirada de Hoerl e Kennard (1970), é possível comparar qualitativamente $\gamma_{1}\left(\lambda^{*}\right), \gamma_{2}\left(\lambda^{*}\right),(3.9)$ e (4.5) em relação ao parâmetro em cristas, $\lambda^{*}$. Podemos notar que, conforme aumentamos o valor de $\lambda^{*}$, aumentamos também o viés do estimador, mas em compensação a soma das variâncias dos estimadores dos parâmetros diminui drasticamente. Já, o erro quadrático médio de $\hat{\beta}_{C}^{*}\left(\lambda^{*}\right)$ se torna menor do que o erro quadrático médio do estimador de mínimos quadrados para alguns valores de $\lambda^{*}$, ou seja, a introdução de um pequeno viés nas estimativas dos parâmetros resulta em uma diminuição do erro quadrático médio.

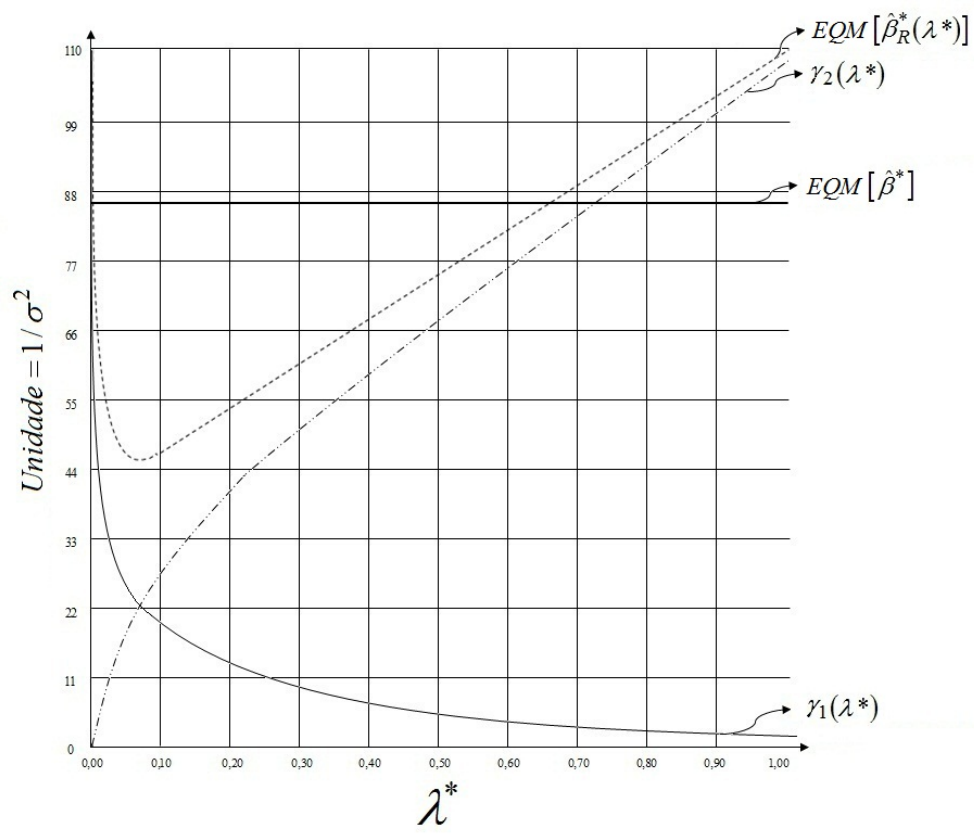

Figura 4.1: Comparação entre a soma das variâncias, o quadrado do viés e os erros quadráticos médios dos estimadores dos parâmetros em cristas e de mínimos quadrados em modelos de regressão linear. Fonte: Hoerl e Kennard (1970).

Essa relação entre os erros quadráticos médios dos estimadores em cristas e de mínimos quadrados é justificada pelas propriedades de $\gamma_{1}\left(\lambda^{*}\right)$ e $\gamma_{2}\left(\lambda^{*}\right)$. A função $\gamma_{1}\left(\lambda^{*}\right)$ é monotônica descrescente em relação à $\lambda^{*}$, enquanto que $\gamma_{2}\left(\lambda^{*}\right)$ é monotônica crescente também em relação à $\lambda^{*}$. Além disso, podemos avaliar os valores das derivadas dessas funções na vizinhança próxima à origem: 


$$
\begin{gathered}
\lim _{\lambda^{*} \rightarrow 0^{+}}\left[\frac{\partial \gamma_{1}\left(\lambda^{*}\right)}{\partial \lambda^{*}}\right]=-2 \sigma^{2} \sum_{j=0}^{s^{*}-1}\left(\frac{1}{\mu_{j}^{* 4}}\right) \\
\lim _{\lambda^{*} \rightarrow 0^{+}}\left[\frac{\partial \gamma_{2}\left(\lambda^{*}\right)}{\partial \lambda^{*}}\right]=0 .
\end{gathered}
$$

Dessa forma, $\gamma_{1}\left(\lambda^{*}\right)$ tem derivada negativa que tende à $-\infty$ conforme $\lambda^{*} \rightarrow 0^{+}$na presença de multicolinearidade. Já $\gamma_{2}\left(\lambda^{*}\right)$ é plana e igual à zero quando $\lambda^{*} \rightarrow 0^{+}$. Com essas considerações, Hoerl e Kennard (1970) chegam à conclusão de que é possível encontrar $\lambda^{*}>0$, ou seja, adicionar um viés no estimador de mínimos quadrados, tal que a redução da variância seja considerável e melhorando ainda o EQM da estimação e previsão.

Em Hoerl e Kennard (1970) é mostrado também que o modelo de regressão linear usual dado por (3.8) pode ser reescrito na sua forma canônica. Fazendo a decomposição espectral de $\left(\mathbf{X}^{*}\right)^{\prime} \mathbf{X}^{*}$ temos que:

$$
\left(\mathrm{X}^{*}\right)^{\prime} \mathbf{X}^{*}=\Theta \Lambda \Theta^{\prime}
$$

em que $\Lambda$ é a matriz diagonal $s \mathrm{x} s$ composta dos autovalores de $\left(\mathbf{X}^{*}\right)^{\prime} \mathbf{X}^{*}$ dados por $\mu_{j}^{2}$ e as colunas de $\Theta$ são os autovetores associados à esses autovalores e são tais que $\Theta^{\prime} \Theta=\mathbf{I}_{s}$, $j=0, \ldots, s-1$. Seja:

$$
\mathbf{X}^{*}=\check{\mathbf{X}}^{*} \Theta^{\prime}
$$

e

$$
\mathbf{Y}^{*}=\check{\mathbf{X}}^{*} \alpha+\epsilon
$$

em que $\alpha=\Theta^{\prime} \beta^{*},\left(\check{\mathbf{X}}^{*}\right)^{\prime} \check{\mathbf{X}}^{*}=\Lambda$ e $\alpha^{\prime} \alpha=\left(\beta^{*}\right)^{\prime} \beta^{*}$. Portanto, o estimador em cristas definido através do modelo de regressão linear na forma canônica será dado por:

$$
\hat{\alpha}_{C}^{*}\left(\Lambda^{*}\right)=\left[\left(\check{\mathbf{X}}^{*}\right)^{\prime} \check{\mathbf{X}}^{*}+\Lambda^{*}\right]^{-1}\left(\check{\mathbf{X}}^{*}\right)^{\prime} \mathbf{Y}^{*}
$$

em que $\Lambda^{*}$ é uma matriz diagonal $s \mathrm{x} s$ composta de $\lambda_{j}^{*}$ que são os parâmetros em cristas, $\lambda_{j}^{*} \geq 0$ e $j=0, \ldots, s-1$. A fórmula dada por (4.7) é mais geral do que a dada por (4.3), pois, para cada covariável, é encontrado um valor de $\lambda_{j}^{*}$ que faz com que o erro quadrático médio seja reduzido. Hoerl e Kennard (1970) citam que é possível mostrar que o valor de $\lambda_{j}^{*}$ que diminui a distância entre $\hat{\alpha}_{C}^{*}\left(\Lambda^{*}\right)$ e $\alpha$ é tal que:

$$
\lambda_{j}^{*}=\frac{\sigma^{2}}{\alpha_{j}^{2}},
$$


em que $\alpha_{j}$ é o j-ésimo componente de $\alpha$. Além disso, (4.8) pode ser aproximado por:

$$
\lambda_{j}^{*}=\frac{\hat{\sigma}^{2}}{\hat{\alpha}_{j}^{2}},
$$

em que $\hat{\sigma}^{2}=\frac{(\mathbf{y}-\hat{\mathbf{y}})^{\prime}(\mathbf{y}-\hat{\mathbf{y}})}{n-s-1}$, ou seja, é o quadrado médio do resíduo e estimador de $\sigma^{2}, \hat{\mathbf{y}}=\hat{\pi}\left(\mathbf{x}_{i}\right)$ é a probabilidade estimada de sucesso do evento e $\hat{\alpha}_{j}^{2}=v_{j} \hat{\beta}$.

\subsubsection{Regressão Logística em Cristas}

Na regressão logística, a ideia para obtenção do estimador em cristas é muito semelhante ao da regressão linear. De acordo com Walker e Duncan (1967), $\hat{\beta}$ minimiza aproximadamente a Soma de Quadrados dos Erros Ponderados (SQEP). Então, segundo Schaefer et al. (1984), o estimador da regressão logística em cristas será o estimador de comprimento mínimo dentre todos os que aumentam SQEP em uma pequena quantia fixada. Utilizando o estimador $\hat{\beta}_{C}(\lambda)$ ao invés de $\hat{\beta}$, os autores comentam que SQEP é aumentado em:

$$
\phi=\left[\hat{\pi}(\hat{\beta})-\hat{\pi}\left(\hat{\beta}_{C}\right)\right]^{\prime} \hat{\mathbf{W}}^{-1}\left[\hat{\pi}(\hat{\beta})-\hat{\pi}\left(\hat{\beta}_{C}\right)\right]+2\left[\hat{\pi}(\hat{\beta})-\hat{\pi}\left(\hat{\beta}_{C}\right)\right]^{\prime} \hat{\mathbf{W}}^{-1}[\mathbf{Y}-\hat{\pi}(\hat{\beta})]
$$

em que $\hat{\pi}(\hat{\beta})$ e $\hat{\pi}\left(\hat{\beta}_{C}\right)$ são as estimativas de $\pi(\mathbf{X})$ usando $\hat{\beta}$ e $\hat{\beta}_{C}(\lambda)$ como estimativas de $\beta$, respectivamente. Escrevendo $\hat{\pi}$ em função de $\hat{\beta}$ em (4.10), os autores verificam que:

$$
\phi=\left[\hat{\beta}-\hat{\beta}_{C}(\lambda)\right]^{\prime} \mathbf{X}^{\prime} \mathbf{W} \mathbf{X}\left[\hat{\beta}-\hat{\beta}_{C}(\lambda)\right]
$$

De forma análoga à regressão linear em cristas, para minimizar $\beta_{C}(\lambda)^{\prime} \beta_{C}(\lambda)$ através do Método de Lagrange com a quantia (4.11) fixada, temos que minimizar:

$$
F=\beta_{C}(\lambda)^{\prime} \beta_{C}(\lambda)+\left(\frac{1}{\lambda}\right)\left\{\left[\hat{\beta}-\beta_{C}(\lambda)\right]^{\prime} \mathbf{X}^{\prime} \hat{\mathbf{W}} \mathbf{X}\left[\hat{\beta}-\beta_{C}(\lambda)\right]-\phi\right\}
$$

em que $1 / \lambda$ é o multiplicador de Lagrange. Derivando (4.12) em relação a $\beta_{C}(\lambda)$, temos:

$$
\frac{\partial F}{\partial \beta_{C}(\lambda)}=2 \beta_{C}(\lambda)+\left(\frac{1}{\lambda}\right)\left[2\left(\mathbf{X}^{\prime} \hat{\mathbf{W}} \mathbf{X}\right) \beta_{C}(\lambda)-2\left(\mathbf{X}^{\prime} \hat{\mathbf{W}} \mathbf{X}\right) \hat{\beta}\right]=0
$$

e como consequência,

$$
\hat{\beta}_{C}(\lambda)=\left(\mathbf{X}^{\prime} \hat{\mathbf{W}} \mathbf{X}+\lambda \mathbf{I}\right)^{-\mathbf{1}}\left(\mathbf{X}^{\prime} \hat{\mathbf{W}} \mathbf{X}\right) \hat{\beta}=C \hat{\beta}, \quad \lambda>0
$$

em que $C=\left(\mathbf{X}^{\prime} \hat{\mathbf{W}} \mathbf{X}+\lambda \mathbf{I}\right)^{-\mathbf{1}}\left(\mathbf{X}^{\prime} \hat{\mathbf{W}} \mathbf{X}\right)$, é o estimador em cristas de $\beta$ na regressão logística. A quantia $\lambda$ será escolhida de maneira a minimizar a restrição (4.11). 
Este estimador é viesado, pois:

$$
E\left[\hat{\beta}_{C}(\lambda)\right]=E[C \hat{\beta}]=C \beta
$$

Já a sua variância é aproximada por:

$$
\begin{aligned}
\widehat{\operatorname{Var}}\left[\hat{\beta}_{C}(\lambda)\right] & =\widehat{\operatorname{Var}}[C \hat{\beta}] \\
& =C^{\prime}\left(\mathbf{X}^{\prime} \hat{\mathbf{W}} \mathbf{X}\right)^{-1} C \\
& =\left(\mathbf{X}^{\prime} \hat{\mathbf{W}} \mathbf{X}+\lambda \mathbf{I}\right)^{-1}\left(\mathbf{X}^{\prime} \hat{\mathbf{W}} \mathbf{X}\right)\left(\mathbf{X}^{\prime} \hat{\mathbf{W}} \mathbf{X}+\lambda \mathbf{I}\right)^{-1}
\end{aligned}
$$

De (4.14) temos que, conforme aumentamos $\lambda$, maior será o viés de $\hat{\beta}_{C}(\lambda)$. Por outro lado, de (4.15), concluímos que a sua variância decresce conforme aumentamos $\lambda$.

O erro quadrático médio de $\hat{\beta}_{C}(\lambda)$ também pode ser calculado. Em Mansson e Shukur (2011), é mostrado que:

$$
\begin{aligned}
\operatorname{EQM}\left(\hat{\beta}_{C}(\lambda)\right) & =E\left[\left(\hat{\beta}_{C}(\lambda)-\beta\right)^{\prime}\left(\hat{\beta}_{C}(\lambda)-\beta\right)\right] \\
& =\sum_{j=0}^{s-1} \frac{\mu_{j}^{2}}{\left(\mu_{j}^{2}+\lambda\right)^{2}}+\lambda^{2} \beta^{\prime}\left[\mathbf{X}^{\prime} \hat{\mathbf{W}} \mathbf{X}+\lambda \mathbf{I}\right]^{-2} \beta \\
& =\gamma_{1}(\lambda)+\gamma_{2}(\lambda)
\end{aligned}
$$

em que $\gamma_{1}(\lambda)=\sum_{j=0}^{s-1} \frac{\mu_{j}^{2}}{\left(\mu_{j}^{2}+\lambda\right)^{2}}$ e $\gamma_{2}(\lambda)=\lambda^{2} \beta^{\prime}\left[\mathbf{X}^{\prime} \hat{\mathbf{W}} \mathbf{X}+\lambda \mathbf{I}\right]^{-2} \beta$. Portanto, de forma análoga à regressão linear em cristas, o erro quadrático médio de $\hat{\beta}_{C}(\lambda)$ é composto pela soma das variâncias dos estimadores dos parâmetros, dado pelo primeiro elemento $\gamma_{1}(\lambda)$, mais o quadrado do erro cometido quando se utiliza o parâmetro $\hat{\beta}_{C}(\lambda)$ ao invés de $\hat{\beta}$, dado pelo segundo elemento $\gamma_{2}(\lambda)$. Note que a soma das variâncias dos estimadores dos parâmetros não está inflacionada, pois $\mu_{j}^{2}$ foi substituído por $\mu_{j}^{2}+\lambda$ no denominador de $\gamma_{1}(\lambda)$.

O objetivo da regressão em cristas é encontrar um valor de $\lambda$ tal que a redução da variância seja maior do que o aumento do viés. Esse valor existe, pois, em Schaefer et al. (1984), é provado que para $n$ grande e dados suficientemente multicolineares, existe um $\lambda_{0}$ tal que o erro quadrático médio do estimador (4.13) é menor do que o erro quadrático médio do estimador de máxima verossimilhança (2.8), ou seja, a introdução de um viés nas estimativas dos parâmetros da regressão logística também resulta em maiores precisões.

Vamos mostrar a seguir algumas formas de se obter o valor de $\lambda$ tal que as estimativas pontuais e da variância dos parâmetros do modelo de regressão logística fiquem mais estáveis.

\subsubsection{Escolha do parâmetro em cristas}

De (4.13) temos que o estimador em cristas depende do parâmetro $\lambda$. Portanto, é preciso fazer a escolha adequada de $\lambda$ para garantirmos que o estimador em cristas seja mais preciso 
do que o estimador de máxima verossimilhança. Em contrapartida, não existem procedimentos automáticos para o cálculo do "melhor" valor de $\lambda$, já que não existem suposições sobre os limites de $\hat{\beta}_{C}(\lambda)$.

De acordo com Hoerl e Kennard (1970), a forma mais adequada de se selecionar o melhor valor de $\lambda$ é analisando o traço de Ridge, um gráfico das estimativas dos coeficientes do modelo como função de vários valores do parâmetro em cristas. Um exemplo deste gráfico pode ser encontrado na Figura 4.1.

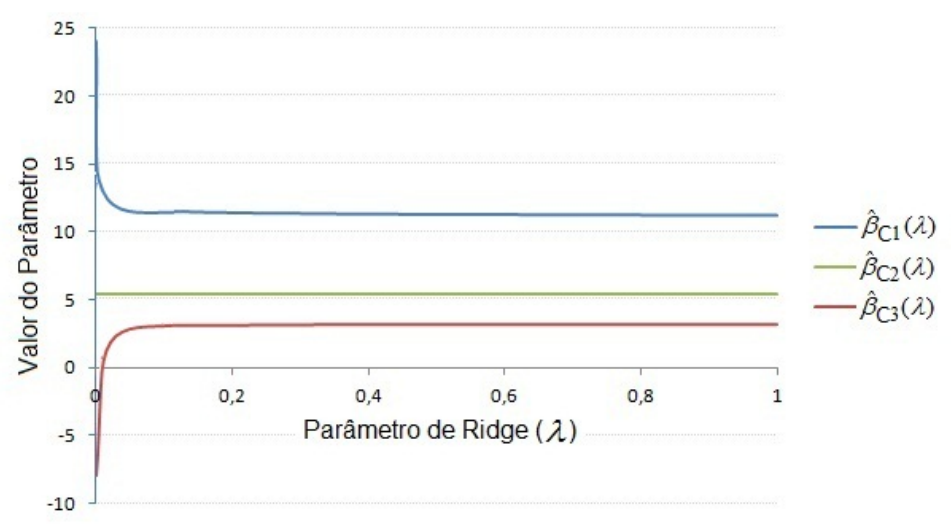

Figura 4.2: Exemplo do gráfico do traço de Ridge.

Neste gráfico, é esperado que as estimativas dos parâmetros associados às covariáveis envolvidas na multicolinearidade e até mesmo seus sinais mudem rapidamente com pequenos incrementos no valor de $\lambda$. A partir de certo valor, essas estimativas irão se estabilizar e mudar gradativamente. Já as estimativas dos parâmetros associados a variáveis não envolvidas na multicolinearidade ficarão sempre estáveis em relação ao aumento de $\lambda$. O parâmetro em cristas escolhido será aquele com menor valor dentre os quais as estimativas de $\hat{\beta}_{C}(\lambda)$ estão estabilizadas. Além disso, elas devem ter ordem de grandeza e sinais conforme o esperado, ou seja, como se não houvesse multicolinaridade nos dados. Um ponto negativo deste método de escolha do parâmetro em cristas é que ele pode ser bastante subjetivo, já que o tamanho do incremento pode variar de procedimento para procedimento e a sensibilidade da estabilização dos parâmetros pode variar de pessoa para pessoa.

Para tentar fugir da subjetividade da escolha de $\lambda$ através da análise do traço de Ridge, vamos sugerir algumas fórmulas para obtenção do valor de $\lambda$. Muitas regras já foram propostas, estudadas e comparadas para a escolha do melhor parâmetro em cristas na regressão linear (como, por exemplo, em Wichern e Churchill (1978)) e partindo das semelhanças entre a regressão linear e a regressão logística, Hoerl e Kennard (1970) propuseram o seguinte parâmetro:

$$
\lambda_{1}=\frac{\hat{\sigma}^{2}}{\hat{\alpha}_{0}^{2}}
$$

em que $\hat{\alpha}_{0}^{2}=\max \left(\hat{\alpha}_{j}^{2}\right)$. O estimador (4.17) foi proposto pela primeira vez por Hoerl e Kennard 
(1970) e é função de (4.9). Outra regra bastante comum é utilizar o valor:

$$
\lambda_{2}=\frac{p}{\hat{\beta}^{\prime} \hat{\beta}},
$$

em que $\hat{\beta}$ é o estimador de máxima verossimilhança de $\beta$. Esse valor é facilmente calculado e foi o que apresentou a maior redução no erro quadrático médio de $\hat{\beta}_{C}(\lambda)$ no estudo empírico apresentado em Schaefer et al. (1984). Além dele, em Mansson e Shukur (2011), foram sugeridas outras possibilidades para o valor de $\lambda$ na regressão logística:

$$
\lambda_{3}=\frac{\hat{\sigma}^{2}}{\left(\prod_{j=0}^{s-1} \hat{\alpha}_{j}^{2}\right)^{1 / s}} .
$$

Se compararmos (4.9) com (4.19), temos que o estimador $\lambda_{3}$ é a média geométrica de (4.9). Os autores propõem ainda o valor:

$$
\lambda_{4}=\operatorname{mediana}\left(\frac{\hat{\sigma}^{2}}{\hat{\alpha}_{j}^{2}}\right), j=0, \ldots, s-1 .
$$

O estimador dado por (4.20) também é função de (4.9). As regras dadas por (4.19) e (4.20) foram propostas pela primeira vez por Kibria (2003). Por fim, em Mansson e Shukur (2011), também foi analisado o estimador dado por:

$$
\lambda_{5}=\max \left(d_{j}\right), j=0, \ldots, s-1,
$$

em que $d_{j}=\frac{\mu_{j} \hat{\sigma}^{2}}{(n-s-1) \hat{\sigma}^{2}+\mu_{j}} \hat{\alpha}_{j}^{2}$, proposto pela primeira vez por Alkhamisi et al. (2006).

\subsection{Regressão em Componentes Principais}

Com o objetivo de reduzir a dimensionalidade do modelo de regressão logística e melhorar as estimativas dos parâmetros quando existe multicolinearidade, outra técnica também muito utilizada é a aplicação de Componentes Principais em modelos de regressão logística. Segundo Gunst e Mason (1980), na regressão linear, esta técnica introduz um pequeno viés nas estimativas dos parâmetros, mas, em compensação, tanto a estimativa pontual quanto a variância dos estimadores dos parâmetros ficam mais estáveis. Apresentaremos a seguir duas formas de calcular este estimador (iterativamente e através do método one-step) e depois decidiremos qual é a mais adequada.

\subsubsection{Estimador em Componentes Principais iterativo}

Recorde que, de (2.8) e (2.11), a expressão para o estimador de $\beta$ e para a sua matriz de covariância dependem de $\mathbf{X}^{\prime} \hat{\mathbf{W}} \mathbf{X}$. Lembre-se também que: 


$$
\mathbf{X}^{\prime} \hat{\mathbf{W}} \mathbf{X}=\sum_{j=0}^{s-1} \mu_{j}^{2} v_{j} v_{j}^{\prime}
$$

e, de acordo com (3.5), temos que:

$$
\left(\mathbf{X}^{\prime} \hat{\mathbf{W}} \mathbf{X}\right)^{-1}=\sum_{j=0}^{s-1} \mu_{j}^{-2} v_{j} v_{j}^{\prime}
$$

Suponha que existam $q$ relações de multicolinearidade entre as covariáveis, ou seja, $\mu_{s-q}, \ldots, \mu_{s-2}, \mu_{s-1}$ são bem pequenos. Dessa forma, a exclusão destes termos da somatória presente em (4.22) não alterará de forma significativa o valor de $\mathbf{X}^{\prime} \hat{\mathbf{W}} \mathbf{X}$ e, consequentemente, pouco da variabilidade total das covariáveis será perdida; em contrapartida, os elementos da matriz apresentada em (4.23) ficarão menores. Assim, temos que:

$$
\left(\mathbf{X}^{\prime} \hat{\mathbf{W}} \mathbf{X}\right)^{-1} \approx \sum_{j=0}^{s-q-1} \mu_{j}^{-2} v_{j} v_{j}^{\prime}
$$

Assumindo ainda que a semente inicial do processo iterativo de estimação de $\beta$ seja igual a zero, ou seja, $\hat{\beta}^{(0)}=0$, baseado em $(2.10)$, podemos escrever:

$$
\begin{aligned}
\hat{\beta}^{(L)} & =\hat{\beta}^{(0)}+\sum_{l=0}^{L}\left(\mathbf{X}^{\prime} \hat{\mathbf{W}}^{(\mathbf{l})} \mathbf{X}\right)^{-\mathbf{1}} \mathbf{X}^{\prime}\left(\mathbf{Y}-\hat{\pi}^{(\mathbf{l})}\right) \\
& =\sum_{l=0}^{L}\left(\mathbf{X}^{\prime} \hat{\mathbf{W}}^{(\mathbf{l})} \mathbf{X}\right)^{-\mathbf{1}} \mathbf{X}^{\prime}\left(\mathbf{Y}-\hat{\pi}^{(\mathbf{l})}\right)
\end{aligned}
$$

em que $L$ é a etapa em que o processo converge.

Assim, de acordo com Schaefer (1986) e Marx e Smith (1990), fazendo a substituição de (4.24) em (4.25), obtemos o estimador dos componentes principais interativo dado por:

$$
\hat{\beta}_{C \text { Pint }}=\sum_{l=0}^{L} \sum_{j=0}^{s-q-1} \mu_{j}^{-2} v_{j} v_{j}^{\prime} \mathbf{X}^{\prime}\left(\mathbf{Y}-\hat{\pi}^{(\mathbf{l})}\right) .
$$

Para simplificar a notação, seja $\left(\mathbf{X}^{\prime} \hat{\mathbf{W}}^{(\mathbf{l})} \mathbf{X}\right)_{(q)}=\sum_{j=0}^{s-q-1} \mu_{j}^{-2} v_{j} v_{j}^{\prime}$. Portanto, temos que:

$$
\hat{\beta}_{C \text { Pint }}=\sum_{l=0}^{L}\left(\mathbf{X}^{\prime} \hat{\mathbf{W}}^{(\mathbf{l})} \mathbf{X}\right)_{(q)} \mathbf{X}^{\prime}\left(\mathbf{Y}-\hat{\pi}^{(\mathbf{l})}\right)
$$


Note que o estimador dado por (4.26) é difícil de se obter, pois a matriz $\left(\mathbf{X}^{\prime} \hat{\mathbf{W}}^{(\mathbf{l})} \mathbf{X}\right)_{(q)}$ deve ser recalculada em cada etapa do processo iterativo e isso envolveria um trabalho computacional bastante grande. Por isso, foi sugerido em Schaefer (1986) um processo alternativo, denominado aqui de one-step, que será apresentado a seguir.

\subsubsection{Estimador em Componentes Principais one-step}

Em Schaefer (1986) é citado que na função logística, se forem fixadas as covariáveis, pequenas mudanças em $\hat{\beta}$ produzem ainda menores mudanças em $\hat{\pi}$ e em $\hat{\mathbf{W}}$. Ainda, temos que $\left(\mathbf{X}^{\prime} \hat{\mathbf{W}} \mathbf{X}\right) \approx\left(\mathbf{X}^{\prime} \hat{\mathbf{W}}^{(\mathbf{l})} \mathbf{X}\right)$ e que $\left(\mathbf{X}^{\prime} \hat{\mathbf{W}} \mathbf{X}\right)_{(q)} \approx\left(\mathbf{X}^{\prime} \hat{\mathbf{W}}^{(\mathbf{l})} \mathbf{X}\right)_{(q)}$. Podemos, portanto, transformar $\hat{\beta}$ de maneira similar à transformação do estimador de mínimos quadrados da regressão em componentes principais, ou seja:

$$
\begin{aligned}
\hat{\beta}_{C P} & =\sum_{l=0}^{L}\left(\mathbf{X}^{\prime} \hat{\mathbf{W}}^{(\mathbf{l})} \mathbf{X}\right)_{(q)} \mathbf{X}^{\prime}\left(\mathbf{Y}-\hat{\pi}^{(\mathbf{l})}\right) \\
& =\sum_{l=0}^{L}\left(\mathbf{X}^{\prime} \hat{\mathbf{W}} \mathbf{X}\right)_{(q)} \mathbf{X}^{\prime}\left(\mathbf{Y}-\hat{\pi}^{(\mathbf{l})}\right) \\
& =\sum_{l=0}^{L}\left(\mathbf{X}^{\prime} \hat{\mathbf{W}} \mathbf{X}\right)_{(q)}\left(\mathbf{X}^{\prime} \hat{\mathbf{W}} \mathbf{X}\right)\left(\mathbf{X}^{\prime} \hat{\mathbf{W}}^{(\mathbf{l})} \mathbf{X}\right)^{-1} \mathbf{X}^{\prime}\left(\mathbf{Y}-\hat{\pi}^{(\mathbf{l})}\right) \\
& =\left(\mathbf{X}^{\prime} \hat{\mathbf{W}} \mathbf{X}\right)_{(q)}\left(\mathbf{X}^{\prime} \hat{\mathbf{W}} \mathbf{X}\right) \sum_{l=0}^{L}\left(\mathbf{X}^{\prime} \hat{\mathbf{W}}^{(\mathbf{l})} \mathbf{X}\right)^{-1} \mathbf{X}^{\prime}\left(\mathbf{Y}-\hat{\pi}^{(\mathbf{l})}\right) \\
& =\left(\mathbf{X}^{\prime} \hat{\mathbf{W}} \mathbf{X}\right)_{(q)}\left(\mathbf{X}^{\prime} \hat{\mathbf{W}} \mathbf{X}\right) \hat{\beta}
\end{aligned}
$$

Portanto, uma simples transformação de $\hat{\beta}$ é aproximadamente igual ao estimador de componentes principais definido em (4.26).

A vantagem de se definir o estimador em componentes principais como:

$$
\hat{\beta}_{C P}=\left(\mathbf{X}^{\prime} \hat{\mathbf{W}} \mathbf{X}\right)_{(q)}\left(\mathbf{X}^{\prime} \hat{\mathbf{W}} \mathbf{X}\right) \hat{\beta}
$$

é que, dessa forma, não é necessário fazer nenhuma modificação em softwares de regressão logística.

O estimador dado por (4.27) é viesado pois: 


$$
\begin{aligned}
E\left[\hat{\beta}_{C P}\right] & =E\left[\left(\mathbf{X}^{\prime} \hat{\mathbf{W}} \mathbf{X}\right)_{(q)}\left(\mathbf{X}^{\prime} \hat{\mathbf{W}} \mathbf{X}\right) \hat{\beta}\right] \\
& =\left(\mathbf{X}^{\prime} \hat{\mathbf{W}} \mathbf{X}\right)_{(q)}\left(\mathbf{X}^{\prime} \hat{\mathbf{W}} \mathbf{X}\right) E[\hat{\beta}] \\
& =\left(\mathbf{X}^{\prime} \hat{\mathbf{W}} \mathbf{X}\right)_{(q)}\left(\mathbf{X}^{\prime} \hat{\mathbf{W}} \mathbf{X}\right) \beta \\
& =\left(\mathbf{I}-\sum_{j=0}^{s-q-1} v_{j} v_{j}^{\prime}\right) \beta \\
& =\beta-\sum_{j=0}^{s-q-1} v_{j} v_{j}^{\prime} \beta
\end{aligned}
$$

uma vez que, utilizando o fato de que $v_{0}, v_{1}, \ldots, v_{s-1}$ são mutuamente ortogonais, temos que:

$$
\begin{aligned}
\left(\mathbf{X}^{\prime} \hat{\mathbf{W}} \mathbf{X}\right)_{(q)}\left(\mathbf{X}^{\prime} \hat{\mathbf{W}} \mathbf{X}\right) & =\sum_{j=0}^{q-1} v_{j} v_{j}^{\prime} \\
& =\mathbf{I}-\sum_{j=s-q}^{s-1} v_{j} v_{j}^{\prime}
\end{aligned}
$$

Já a sua variância é dada por:

$$
\begin{aligned}
\operatorname{Var}\left[\hat{\beta}_{C P}\right] & =\operatorname{Var}\left[\left(\mathbf{X}^{\prime} \hat{\mathbf{W}} \mathbf{X}\right)_{(q)}\left(\mathbf{X}^{\prime} \hat{\mathbf{W}} \mathbf{X}\right) \hat{\beta}\right] \\
& =\left(\mathbf{X}^{\prime} \hat{\mathbf{W}} \mathbf{X}\right)_{(q)}\left(\mathbf{X}^{\prime} \hat{\mathbf{W}} \mathbf{X}\right) \operatorname{Var}[\hat{\beta}]\left(\mathbf{X}^{\prime} \hat{\mathbf{W}} \mathbf{X}\right)\left(\mathbf{X}^{\prime} \hat{\mathbf{W}} \mathbf{X}\right)_{(q)} \\
& =\left(\mathbf{X}^{\prime} \hat{\mathbf{W}} \mathbf{X}\right)_{(q)}\left(\mathbf{X}^{\prime} \hat{\mathbf{W}} \mathbf{X}\right)\left(\mathbf{X}^{\prime} \hat{\mathbf{W}} \mathbf{X}\right)^{-1}\left(\mathbf{X}^{\prime} \hat{\mathbf{W}} \mathbf{X}\right)\left(\mathbf{X}^{\prime} \hat{\mathbf{W}} \mathbf{X}\right)_{(q)} \\
& =\left(\mathbf{X}^{\prime} \hat{\mathbf{W}} \mathbf{X}\right)_{(q)}\left(\mathbf{X}^{\prime} \hat{\mathbf{W}} \mathbf{X}\right)\left(\mathbf{X}^{\prime} \hat{\mathbf{W}} \mathbf{X}\right)_{(q)} \\
& =\left(\mathbf{X}^{\prime} \hat{\mathbf{W}} \mathbf{X}\right)_{(q)} \\
& =\sum_{j=0}^{s-q-1} \mu_{j}^{-2} v_{j} v_{j}^{\prime},
\end{aligned}
$$

pois, de acordo com Gunst e Mason (1980) e utilizando novamente que $v_{0}, v_{1}, \ldots, v_{s-1}$ são mutuamente ortogonais, temos que:

$$
\left(\mathbf{X}^{\prime} \hat{\mathbf{W}} \mathbf{X}\right)_{(q)}\left(\mathbf{X}^{\prime} \hat{\mathbf{W}} \mathbf{X}\right)\left(\mathbf{X}^{\prime} \hat{\mathbf{W}} \mathbf{X}\right)_{(q)}=\left(\mathbf{X}^{\prime} \hat{\mathbf{W}} \mathbf{X}\right)_{(q)} .
$$

Além disso, temos que o erro quadrático médio de $\hat{\beta}_{C P}$, dado por $E Q M\left(\hat{\beta}_{C P}\right)$, de acordo com Marx e Smith (1990), é igual a:

$$
\operatorname{EQM}\left(\hat{\beta}_{C P}\right)=\sum_{j=0}^{s-1} \sum_{k=0}^{s-q-1} v_{k j}^{2} \mu_{k}^{-2}+\sum_{j=0}^{s-1}\left(\sum_{k=s-q}^{s-1} v_{k}^{\prime} \beta v_{j k}\right)^{2}
$$


Portanto, temos que desconsiderando os $q$ menores autovalores obtidos da decomposição espectral da matriz $\mathbf{X}^{\prime} \hat{\mathbf{W}} \mathbf{X}$, obtemos os componentes principais que fazem com que a variância do estimador $\hat{\beta}_{C P}$ fique significativamente menor do que a variância do estimador de máxima verossimilhança de $\beta$. Vale ressaltar também que como o estimador dado por (4.27) é fácil de ser obtido e não precisa ser recalculado em cada etapa do processo iterativo de estimação de $\beta$, então o método one-step será preferido.

\subsubsection{Exclusão dos componentes principais}

O estimador dado por (4.27) considera que existem $q$ multicolinearidades entre as covariáveis do modelo de regressão logística, ou seja, foram eliminados os $q$ componentes principais que menos representavam a variabilidade dos dados e que tornavam as estimativas pontuais e das variâncias dos parâmetros superstimadas. Para se determinar qual é a quantidade ideal de componentes principais que devem ser retirados, Marx e Smith (1990) comenta que uma simples estratégia é deletar todos os componentes associados à pequenos autovalores. Dessa forma, sugerimos os critérios utilizando o índice de condição ou o número de condição apresentado no Capítulo 3.2.2.

Um outro critério mais sofisticado também exposto em Marx e Smith (1990) e que foi apresentado por Hill et al. (1977) considera a exclusão dos componentes principais tal que $\hat{\beta}_{C P}$ fique o mais próximo possível de $\beta$, ou seja, $\hat{\beta}_{C P}$ será preferido em relação à $\hat{\beta}$ se:

$$
\operatorname{tr}\left[E Q M\left(\hat{\beta}_{C P}\right)\right] \leq \operatorname{tr}[E Q M(\hat{\beta})]
$$

\subsection{Considerações finais do capítulo}

A estimação dos parâmetros dos modelos de regressão logística via máxima verossimilhança é, sem dúvida, a mais versátil e poderosa metodologia já desenvolvida, não somente pelas suas propriedades bem estabelecidas, como a sua distribuição, mas também pelas inúmeras ferramentas de ajuste do modelo, como técnicas de seleção de covariáveis, procedimentos de avaliação da especificação do modelo, etc. Por todos esses motivos, a técnica de máxima verossimilhança deve ser preferida na análise dos dados.

A escolha desta técnica deve ser repensada, porém, quando a multicolinearidade entre as covariáveis está presente. Em situações como esta, os estimadores viesados devem ser levados em consideração. Apesar da relativa escassez de teoria sobre a exata distribuição destes estimadores, eles podem gerar estimativas dos parâmetros mais realistas e mais precisas em relação às de máxima verossimilhança.

Neste capítulo foi mostrado que os dois estimadores viesados apresentados diminuem drasticamente a estimativa da variância dos estimadores dos parâmetros, deixando-os mais estáveis em relação ao de máxima verossimilhança. Porém, cada um desses estimadores alternativos apresenta um ponto negativo. Não existem procedimentos automáticos para 
obtenção do "melhor" valor do parâmetro do estimador em cristas, fazendo com que ele não seja otimizado. O ponto negativo do estimador em componentes principais é que os autovetores envolvidos na multicolinearidade devem ser removidos do estimador.

No próximo capítulo apresentaremos um estudo de simulação para identificar em quais condições os estimadores em cristas e em componentes principais apresentam um melhor desempenho em relação ao de máxima verossimilhança. 
42 ESTIMADORES ALTERNATIVOS PARA OS PARÂMETROS DO MODELO DE REGRESSÃO LOGÍSTICA 


\section{Capítulo 5}

\section{Estudo de Simulação}

Neste capítulo será exposto o estudo de simulação realizado com o objetivo de identificar em quais condições os estimadores apresentados anteriormente conseguem reduzir de forma drástica o erro quadrático médio do estimador de $\beta$. Foi analisada a eficiência desses estimadores em função do tamanho da amostra, da quantidade de covariáveis no modelo de regressão logística e do grau de multicolinearidade entre as covariáveis.

\subsection{Descrição dos cenários e modelo}

Neste estudo de simulação foram investigadas as seguintes características nos seguintes níveis:

- Tamanho da amostra (Amostra): 100, 500 e 1000;

- Quantidade de covariáveis (Covars): 5, 10 e 15;

- Grau de multicolinearidade (Multicol): dependendo da quantidade de covariáveis, foram analisados alguns níveis de multicolinearidade. Nos casos em que se tinha 5 covariáveis, foram considerados os graus "nenhum", "moderado" e "severo 1". Nos casos em que se tinham 10 covariáveis, foi gerado mais um cenário denominado de "severo 2" e nos casos em que existiam 15 covariáveis no modelo, foram acrescentados mais dois níveis denominados de "severo 2" e "severo 3".

O grau de multicolinearidade corresponde ao valor máximo de $R_{j}^{2}$ dentre as covariáveis, semelhante a como foi utilizado em Schaefer (1986). O método de classificação nas categorias "nenhum", "moderado", "severo 1", "severo 2" e "severo 3" será discutido posteriormente.

Foram estudadas todas as combinações de tamanho da amostra com quantidade de covariáveis e o grau de multicolinearidade que dependia da quantidade de covariáveis, totalizando 36 cenários diferentes e cada cenário foi replicado 1000 vezes, $r=1, \ldots, 1000$.

O modelo utilizado na simulação foi o de regressão logística dado por: 


$$
\pi\left(\mathbf{x}_{i}\right)=\frac{\exp \left(\mathbf{x}_{i}^{\prime} \beta\right)}{1+\exp \left(\mathbf{x}_{i}^{\prime} \beta\right)}, i=1,2, \ldots, n,
$$

em que $\pi\left(\mathbf{x}_{\mathbf{i}}\right)$ é a probabilidade de sucesso do evento para o i-ésimo indivíduo, $Y_{i}$ é o valor da variável resposta binária do i-ésimo elemento amostral e $\beta=\left(\beta_{0}, \beta_{1}, \ldots, \beta_{p-1}\right)^{\prime}$ é um vetor $p$ x1 de parâmetros desconhecidos associados às covariáveis, $n=100,500$ ou 1000 e $p=5$, 10 ou 15.

\subsection{Delineamento do estudo de simulação}

Para cada uma das réplicas, o processo a seguir foi adotado, baseado no que foi apresentado em Schaefer (1986). O programa foi elaborado utilizando o software R versão 2.14 .2 e pode ser encontrado no Apêndice B.

O primeiro passo foi gerar todos os valores das covariáveis presentes na matriz de planejamento $\mathbf{X}$ de forma aleatória seguindo uma distribuição Uniforme $(0,1)$. Em seguida, o grau de multicolinearidade foi introduzido. Para aquelas simulações sem multicolinearidade, a matriz $\mathbf{X}$ foi mantida intacta. Já para os outros níveis, a multicolinearidade foi inserida substituindo a quinta, a décima e a décima quinta covariável, quando se aplicava, por uma combinação linear das 2 covariáveis anteriores. Dessa forma, para a quinta, a décima e a décima quinta covariável, foi utilizada a seguinte fórmula:

$$
x_{i p}=x_{i p-1}+x_{i p-2}+c \cdot U(0,1), i=1,2, \ldots, n, p=5,10,15 \text { e } c=0,45 \text { ou } 0,142,
$$

em que $U(0,1)$ é uma variável aleatória com distribuição Uniforme $(0,1)$. A adição da perturbação $c$ na criação dessas covariáveis faz com que a matriz de correlação teórica entre as covariáveis no caso em que $p=5$, de acordo com Schaefer (1986), fique igual a:

$$
\left(\begin{array}{lllll}
1 & 0 & 0 & 0 & 0 \\
0 & 1 & 0 & 0 & 0 \\
0 & 0 & 1 & 0 & \rho \\
0 & 0 & 0 & 1 & \rho \\
0 & 0 & \rho & \rho & 1
\end{array}\right)
$$

em que $\rho=0,6738$ quando $c=0,45$ e $\rho=0,7036$ quando $c=0,142$. No caso de 10 covariáveis, a matriz de correlação teórica fica igual a: 


$$
\left(\begin{array}{llllllllll}
1 & 0 & 0 & 0 & 0 & 0 & 0 & 0 & 0 & 0 \\
0 & 1 & 0 & 0 & 0 & 0 & 0 & 0 & 0 & 0 \\
0 & 0 & 1 & 0 & \rho & 0 & 0 & 0 & 0 & 0 \\
0 & 0 & 0 & 1 & \rho & 0 & 0 & 0 & 0 & 0 \\
0 & 0 & \rho & \rho & 1 & 0 & 0 & 0 & 0 & 0 \\
0 & 0 & 0 & 0 & 0 & 1 & 0 & 0 & 0 & 0 \\
0 & 0 & 0 & 0 & 0 & 0 & 1 & 0 & 0 & 0 \\
0 & 0 & 0 & 0 & 0 & 0 & 0 & 1 & 0 & \rho \\
0 & 0 & 0 & 0 & 0 & 0 & 0 & 0 & 1 & \rho \\
0 & 0 & 0 & 0 & 0 & 0 & 0 & \rho & \rho & 1
\end{array}\right) .
$$

Já no caso de 15 covariáveis, a matriz de correlação fica dada por:

$$
\left(\begin{array}{lllllllllllllll}
1 & 0 & 0 & 0 & 0 & 0 & 0 & 0 & 0 & 0 & 0 & 0 & 0 & 0 & 0 \\
0 & 1 & 0 & 0 & 0 & 0 & 0 & 0 & 0 & 0 & 0 & 0 & 0 & 0 & 0 \\
0 & 0 & 1 & 0 & \rho & 0 & 0 & 0 & 0 & 0 & 0 & 0 & 0 & 0 & 0 \\
0 & 0 & 0 & 1 & \rho & 0 & 0 & 0 & 0 & 0 & 0 & 0 & 0 & 0 & 0 \\
0 & 0 & \rho & \rho & 1 & 0 & 0 & 0 & 0 & 0 & 0 & 0 & 0 & 0 & 0 \\
0 & 0 & 0 & 0 & 0 & 1 & 0 & 0 & 0 & 0 & 0 & 0 & 0 & 0 & 0 \\
0 & 0 & 0 & 0 & 0 & 0 & 1 & 0 & 0 & 0 & 0 & 0 & 0 & 0 & 0 \\
0 & 0 & 0 & 0 & 0 & 0 & 0 & 1 & 0 & \rho & 0 & 0 & 0 & 0 & 0 \\
0 & 0 & 0 & 0 & 0 & 0 & 0 & 0 & 1 & \rho & 0 & 0 & 0 & 0 & 0 \\
0 & 0 & 0 & 0 & 0 & 0 & 0 & \rho & \rho & 1 & 0 & 0 & 0 & 0 & 0 \\
0 & 0 & 0 & 0 & 0 & 0 & 0 & 0 & 0 & 0 & 1 & 0 & 0 & 0 & 0 \\
0 & 0 & 0 & 0 & 0 & 0 & 0 & 0 & 0 & 0 & 0 & 1 & 0 & 0 & 0 \\
0 & 0 & 0 & 0 & 0 & 0 & 0 & 0 & 0 & 0 & 0 & 0 & 1 & 0 & \rho \\
0 & 0 & 0 & 0 & 0 & 0 & 0 & 0 & 0 & 0 & 0 & 0 & 0 & 1 & \rho \\
0 & 0 & 0 & 0 & 0 & 0 & 0 & 0 & 0 & 0 & 0 & 0 & \rho & \rho & 1
\end{array}\right) .
$$

Se dentre as covariáveis com multicolinearidade do modelo tivesse pelo menos uma em que a constante de pertubação fosse igual a 0,142 , então o valor máximo de $R_{j}^{2}$ era igual a $99 \%$; caso contrário, o valor máximo de $R_{j}^{2}$ era igual a $90 \%$. No primeiro caso, o nível de multicolinearidade foi classificado como "severo". Dessa forma, quando $j=5$, o nível de multicolinearidade foi classificado como "severo 1". Já quando $j=10$, consideramos duas combinações possíveis:

1. $\mathbf{x}_{5}$ construída de tal forma que $c=0,45$ e $\mathbf{x}_{10}$ construída de tal forma que $c=0,142$;

2. $\mathbf{x}_{5}$ e $\mathbf{x}_{10}$ construídas de tal forma que $c=0,142$.

Esses dois casos foram classificados como "severo 1" e "severo 2", respectivamente. Por fim, quando $j=15$, consideramos três combinações possíveis: 
1. $\mathbf{x}_{5}$ e $\mathbf{x}_{10}$ construídas de tal forma que $c=0,45$ e $\mathbf{x}_{15}$ construída de tal forma que $c=0,142$;

2. $\mathbf{x}_{5}$ construída de tal forma que $c=0,45$ e $\mathbf{x}_{10}$ e $\mathbf{x}_{15}$ construídas de tal forma que $c=0,142$;

3. $\mathbf{x}_{5}, \mathbf{x}_{10}$ e $\mathbf{x}_{15}$ construídas de tal forma que $c=0,142$.

O nível de multicolinearidade considerado nesses três casos foi "severo 1", "severo 2" e "severo 3", respectivamente. Quando todas as constantes de perturbação das covariáveis com multicolinearidade eram iguais a 0,45 , o nível foi considerado como "moderado" e quando todas as constantes de perturbação eram iguais a zero, o nível foi considerado como " nenhum".

A matrix $\mathbf{X}$ foi então multiplicada por 10. Isso foi feito com o objetivo de garantir a variabilidade de $\pi\left(\mathbf{x}_{i}\right)$. Se a matriz $\mathbf{X}$ não fosse alterada, os valores de $\pi\left(\mathbf{x}_{i}\right)$ seriam praticamente iguais e, consequentemente, os resultados da simulação poderiam favorecer algum dos estimadores.

Depois de padronizar as covariáveis, de acordo com (2.1), a probabilidade $\pi\left(\mathbf{x}_{i}\right)$ foi calculada assumindo, sem perda de generalidade, que todos os $\beta_{j}$ 's, $j=0, \ldots, s-1$, eram iguais a um. A variável resposta foi então gerada independentemente através de uma distribuição Bernoulli com parâmetro igual a $\pi\left(\mathbf{x}_{i}\right)$. Por fim, os três estimadores descritos no Capítulo 4 e a estimativa de seus erros quadráticos médios foram calculados. O erro quadrático médio estimado de cada estimador é dado por:

$$
\begin{aligned}
\widehat{E Q M}(\hat{\beta}) & =\sum_{r=1}^{1000} \frac{1}{1000}\left(\mathbf{1}_{p}-\hat{\beta}^{r}\right)^{\prime}\left(\mathbf{1}_{p}-\hat{\beta}^{r}\right) \\
\widehat{E Q M}\left(\hat{\beta}_{C}(\lambda)\right) & =\sum_{r=1}^{1000} \frac{1}{1000}\left(\mathbf{1}_{p}-\hat{\beta}_{C}^{r}\right)^{\prime}\left(\mathbf{1}_{p}-\hat{\beta}_{C}^{r}\right) \\
\widehat{E Q M}\left(\hat{\beta}_{C P}\right) & =\sum_{r=1}^{1000} \frac{1}{1000}\left(\mathbf{1}_{p}-\hat{\beta}_{C P}^{r}\right)^{\prime}\left(\mathbf{1}_{p}-\hat{\beta}_{C P}^{r}\right),
\end{aligned}
$$

em que $\mathbf{1}_{p}$ é o vetor de 1's com dimensão $p$ e $\hat{\beta}^{r}, \hat{\beta}_{C}^{r}$ e $\hat{\beta}_{C P}^{r}$ são os estimadores de máxima verossimilhança, em cristas e em componentes principais na r-ésima réplica do estudo de simulação, respectivamente, $r=1, \ldots, 1000$.

Para o estimador em cristas foram contabilizados os cinco valores para o parâmetro $\lambda$ descritos em (4.17) a (4.21) e aquele que fornecesse o menor erro quadrático médio era o escolhido. Já, o estimador em componentes principais era tal que todos os autovalores vinculados com os índices de condição maiores ou iguais a dez eram excluídos do estimador dado por (4.27). Dessa forma, se o índice de condição da matriz de planejamento da simulação fosse menor do que dez, esse estimador era igual ao de máxima verossimilhança.

O processo descrito até aqui foi replicado 1000 vezes para cada um dos 36 cenários. Foi colocada uma condição no modelo de regressão logística quando os parâmetros eram estimados através de máxima verossimilhança de que se o processo de estimação não convergisse ou 
se o erro quadrático médio fosse maior do que 1000000, outra matriz $\mathbf{X}$ e, consequentemente, outros valores para a variável resposta eram gerados. Essa condição foi colocada para que não tivéssemos problemas com a convergência no processo de estimação dos parâmetros do modelo. Quando isso ocorria, essa réplica não era contabilizada dentre as 1000.

Todos os conjuntos de dados gerados nesse estudo, assim como os índices de condição, os valores máximos de $R_{j}^{2}, j=1, \ldots, p$-1, os valores dos coeficientes dos três modelos, os valores dos parâmetros em cristas e os seus erros quadráticos médios foram armazenados e estão à disposição caso necessário.

\subsection{Resultados}

Para avaliar o nível da multicolinearidade, foram considerados o índice de condição e o valor máximo de $R_{j}^{2}$ de cada simulação e cenário. Na Tabela 5.1 podem ser encontrados os seus valores médios e os seus respectivos erros padrão. Analisando essa tabela podemos concluir que:

- de fato, independente do tamanho da amostra e da quantidade de covariáveis, as médias do máximo valor de $R_{j}^{2}$ obtidos dos cenários com multicolinearidade moderada são próximos a $90 \%$ e os cenários com multicolinearidade severa (1, 2 ou 3) apresentam valores do máximo $R_{j}^{2}$ muito próximos à $99 \%$;

- dentre os cenários com multicolinearidade severa, os cenários classificados como "severo 3" possuem índice de condição levemente superiores ao dos classificados como "severo 1" e "severo 2". Nos cenários em que não há multicolinearidade, tanto as médias dos valores máximo de $R_{j}^{2}$ quanto as médias dos índices de condição são muito pequenos;

- conforme aumentamos o tamanho da amostra, os valores do índice de condição e do máximo $R_{j}^{2}$ ficam mais estáveis e levemente menores;

- quando aumentamos a quantidade de covariáveis, maiores ficam os valores médios do índice de condição e da média do valor máximo de $R_{j}^{2}$. 


\begin{tabular}{|c|c|c|c|c|}
\hline $\begin{array}{l}\text { Tam. da } \\
\text { amostra }\end{array}$ & $\begin{array}{l}\text { Quant. de } \\
\text { covariáveis }\end{array}$ & $\begin{array}{l}\text { Nível de } \\
\text { multicol. }\end{array}$ & $\begin{array}{c}\max \left(R_{j}^{2}\right) \\
\text { média (e.p.) }\end{array}$ & $\begin{array}{c}\eta \\
\text { média (e.p.) }\end{array}$ \\
\hline \multirow{12}{*}{100} & \multirow{3}{*}{5} & nenhum & $0,066(0,031)$ & $2,373(0,471)$ \\
\hline & & moderado & $0,91(0,014)$ & $6,393(0,725)$ \\
\hline & & severo 1 & $0,99(0,002)$ & $19,627(2,08)$ \\
\hline & \multirow{4}{*}{10} & nenhum & $0,152(0,038)$ & $4,518(3,074)$ \\
\hline & & moderado & $0,923(0,01)$ & $11,25(7,992)$ \\
\hline & & severo 1 & $0,991(0,001)$ & $28,72(11,622)$ \\
\hline & & severo 2 & $0,992(0,001)$ & $31,79(11,835)$ \\
\hline & \multirow{5}{*}{15} & nenhum & $0,231(0,04)$ & $12,357(23,03)$ \\
\hline & & moderado & $0,93(0,008)$ & $28,32(66,165)$ \\
\hline & & severo 1 & $0,991(0,001)$ & $50,747(61,517)$ \\
\hline & & severo 2 & $0,992(0,001)$ & $58,62(89,735)$ \\
\hline & & severo 3 & $0,992(0,001)$ & $67,776(80,195)$ \\
\hline \multirow{12}{*}{500} & \multirow{3}{*}{5} & nenhum & $0,013(0,006)$ & $1,987(0,14)$ \\
\hline & & moderado & $0,909(0,006)$ & $5,798(0,288)$ \\
\hline & & severo 1 & $0,99(0,001)$ & $17,918(0,855)$ \\
\hline & \multirow{4}{*}{10} & nenhum & $0,031(0,008)$ & $2,654(0,231)$ \\
\hline & & moderado & $0,913(0,005)$ & $7,277(0,39)$ \\
\hline & & severo 1 & $0,99(0,001)$ & $21,627(1,142)$ \\
\hline & & severo 2 & $0,991(0,001)$ & $22,501(1,172)$ \\
\hline & \multirow{5}{*}{15} & nenhum & $0,047(0,009)$ & $3,321(0,322)$ \\
\hline & & moderado & $0,915(0,004)$ & $7,904(0,4)$ \\
\hline & & severo 1 & $0,99(0,001)$ & $22,696(1,192)$ \\
\hline & & severo 2 & $0,991(0,001)$ & $23,589(1,199)$ \\
\hline & & severo 3 & $0,991(0)$ & $24,437(1,285)$ \\
\hline \multirow{12}{*}{1000} & \multirow{3}{*}{5} & nenhum & $0,007(0,003)$ & $1,933(0,095)$ \\
\hline & & moderado & $0,908(0,004)$ & $5,741(0,188)$ \\
\hline & & severo 1 & $0,99(0)$ & $17,751(0,603)$ \\
\hline & \multirow{4}{*}{10} & nenhum & $0,016(0,004)$ & $2,502(0,146)$ \\
\hline & & moderado & $0,911(0,003)$ & $7,046(0,243)$ \\
\hline & & severo 1 & $0,99(0)$ & $21,178(0,781)$ \\
\hline & & severo 2 & $0,99(0)$ & $21,877(0,785)$ \\
\hline & \multirow{5}{*}{15} & nenhum & $0,024(0,005)$ & $3,027(0,194)$ \\
\hline & & moderado & $0,913(0,003)$ & $7,385(0,267)$ \\
\hline & & severo 1 & $0,99(0)$ & $21,702(0,77)$ \\
\hline & & severo 2 & $0,99(0)$ & $22,39(0,757)$ \\
\hline & & severo 3 & $0,991(0)$ & $22,873(0,77)$ \\
\hline
\end{tabular}

Tabela 5.1: Média e erro padrão do indice de condição e do valor máximo do coeficiente de determinação de cada cenário. 
Na Tabela 5.2 podemos encontrar os valores das estimativas dos erros quadráticos médios e seus erros padrão referente aos três estimadores. Nota-se que:

- não houve redução significativa do erro quadrático médio dos estimadores viesados em relação ao estimador de máxima verossimilhança nas simulações sem multicolinearidade. Esse fato não foi válido somente para os cenários com tamanho de amostra igual a 100, em que o estimador em cristas apresentou o erro quadrático médio inferior aos demais;

- independente do tamanho da amostra e da quantidade de covariáveis, nos cenários com multicolinearidade moderada temos um maior ganho em utilizar o estimador em cristas. Em contrapartida, nos cenários severos (1, 2 ou 3), em geral, há uma maior redução no erro quadrático médio utilizando o estimador em componentes principais. Em todos esses cenários houve uma redução significativa tanto da estimativa pontual quanto no erro padrão referente ao erro quadrático médio dos estimadores viesados em relação ao de máxima verossimilhança;

- conforme aumentamos o tamanho da amostra, os valores dos erros quadráticos médios ficam mais estáveis e menores. Quando o tamanho da amostra é igual a 100, o estimador de máxima verossimilhança fica com o erro quadrático médio e com o erro padrão muito superior aos demais estimadores, principalmente nos cenários com 15 covariáveis. Além disso, utilizando os estimadores viesados, percebe-se uma maior redução do erro quadrático médio nas simulações com tamanho de amostra igual a 100 do que nas iguais a 500 ou 1000;

- quando aumentamos a quantidade de covariáveis, maiores ficam os valores dos erros quadráticos médios, o que faz sentido, já que estamos somando os erros referentes à uma maior quantidade de covariáveis. 


\begin{tabular}{|c|c|c|c|c|c|}
\hline $\begin{array}{l}\text { Tam. da } \\
\text { amostra }\end{array}$ & $\begin{array}{l}\text { Quant. de } \\
\text { covariáveis }\end{array}$ & $\begin{array}{l}\text { Nível de } \\
\text { multicol. }\end{array}$ & $\begin{array}{c}E \hat{Q} M(\hat{\beta}) \\
\text { média (e.p.) }\end{array}$ & $\begin{array}{c}E \hat{Q} M\left(\hat{\beta}_{C}(\lambda)\right) \\
\text { média (e.p.) }\end{array}$ & $\begin{array}{l}E \hat{Q} M\left(\hat{\beta}_{C P}\right) \\
\text { média (e.p.) }\end{array}$ \\
\hline \multirow{12}{*}{100} & \multirow{3}{*}{5} & nenhum & $0,97(1,66)$ & $0,49(0,53)$ & $0,97(1,66)$ \\
\hline & & moderado & $3,89(5,09)$ & $1,08(1,4)$ & $3,89(5,09)$ \\
\hline & & severo 1 & $27,84(41,08)$ & $3,52(8,03)$ & $0,98(1,71)$ \\
\hline & \multirow{4}{*}{10} & nenhum & $8,23(50,18)$ & $1,19(0,79)$ & $5,09(6,8)$ \\
\hline & & moderado & $38,45(206)$ & $2,77(2,92)$ & $7,67(6,03)$ \\
\hline & & severo 1 & $130,17(673,78)$ & $7,22(17,03)$ & $6,19(5,88)$ \\
\hline & & severo 2 & $196,77(558,61)$ & $12,25(27,58)$ & $3,46(4,56)$ \\
\hline & \multirow{5}{*}{15} & nenhum & $87,39(505,8)$ & $2,39(1,5)$ & $10,87(6,88)$ \\
\hline & & moderado & $292,04(1550,46)$ & $5,49(6,44)$ & $8,89(4,48)$ \\
\hline & & severo 1 & $689,77(3561,37)$ & $9,01(15,65)$ & $8,08(4,7)$ \\
\hline & & severo 2 & $1028,32(9471,76)$ & $11,53(16,16)$ & $7,28(4,91)$ \\
\hline & & severo 3 & $1380,37(5920,41)$ & $16,57(35,21)$ & $6,49(5,41)$ \\
\hline \multirow{12}{*}{500} & \multirow{3}{*}{5} & nenhum & $0,13(0,1)$ & $0,1(0,08)$ & $0,13(0,1)$ \\
\hline & & moderado & $0,54(0,59)$ & $0,33(0,35)$ & $0,54(0,59)$ \\
\hline & & severo 1 & $3,91(5,27)$ & $0,77(1,53)$ & $0,21(0,11)$ \\
\hline & \multirow{4}{*}{10} & nenhum & $0,34(0,24)$ & $0,23(0,12)$ & $0,34(0,24)$ \\
\hline & & moderado & $1,48(1,3)$ & $0,76(0,61)$ & $1,48(1,3)$ \\
\hline & & severo 1 & $6,35(8,31)$ & $1,21(1,79)$ & $0,94(0,86)$ \\
\hline & & severo 2 & $10,3(10,28)$ & $1,57(2,21)$ & $0,5(0,29)$ \\
\hline & \multirow{5}{*}{15} & nenhum & $0,64(0,51)$ & $0,37(0,17)$ & $0,64(0,51)$ \\
\hline & & moderado & $2,98(2,11)$ & $1,27(0,75)$ & $2,97(2,05)$ \\
\hline & & severo 1 & $8,67(9,61)$ & $1,54(1,45)$ & $2,19(1,86)$ \\
\hline & & severo 2 & $15,19(14,64)$ & $2,05(2,53)$ & $1,48(1,21)$ \\
\hline & & severo 3 & $22,18(20,77)$ & $2,91(4,66)$ & $0,91(0,62)$ \\
\hline \multirow{12}{*}{1000} & \multirow{3}{*}{5} & nenhum & $0,06(0,05)$ & $0,06(0,04)$ & $0,06(0,05)$ \\
\hline & & moderado & $0,26(0,3)$ & $0,19(0,22)$ & $0,26(0,3)$ \\
\hline & & severo 1 & $2,04(2,76)$ & $0,57(0,97)$ & $0,14(0,05)$ \\
\hline & \multirow{4}{*}{10} & nenhum & $0,16(0,11)$ & $0,12(0,07)$ & $0,16(0,11)$ \\
\hline & & moderado & $0,64(0,52)$ & $0,44(0,34)$ & $0,64(0,52)$ \\
\hline & & severo 1 & $2,69(3,11)$ & $0,67(0,77)$ & $0,48(0,37)$ \\
\hline & & severo 2 & $4,89(4,73)$ & $1,06(1,36)$ & $0,32(0,11)$ \\
\hline & \multirow{5}{*}{15} & nenhum & $0,28(0,18)$ & $0,19(0,09)$ & $0,28(0,18)$ \\
\hline & & moderado & $1,26(0,92)$ & $0,77(0,52)$ & $1,26(0,92)$ \\
\hline & & severo 1 & $3,85(4,49)$ & $1,01(0,98)$ & $1,01(0,73)$ \\
\hline & & severo 2 & $6,65(6,3)$ & $1,32(1,43)$ & $0,75(0,5)$ \\
\hline & & severo 3 & $9,26(7,27)$ & $1,67(1,68)$ & $0,53(0,18)$ \\
\hline
\end{tabular}

Tabela 5.2: Média e erro padrão das estimativas do erro quadrático médio do estimador em cada modelo e cenário. 
Já, na Tabela 5.3, podemos encontrar a quantidade e o percentual de vezes em que cada estimador apresentou o menor erro quadrático médio nos 36 cenários. Se o erro quadrático do estimador de máxima verossimilhança fosse igual a pelo menos um dos demais estimadores, era contabilizado como se aquele fosse o menor. Conclui-se que:

- independente do tamanho da amostra e da quantidade de covariáveis, nos cenários em que se tinha nenhuma multicolinearidade ou multicolinearidade moderada, o estimador em cristas apresentou a maior quantidade de simulações com menor erro quadrático. Nos cenários com multicolinearidade "severa 1" isso também se repetiu, porém somente quando se tinham 10 ou 15 covariáveis; com cinco covariáveis o estimador que apresentou a maior quantidade de simulações com menor erro quadrático foi o de componentes principais. Nos cenários com multicolinearidade "severa 2" e "severa 3", o estimador em componentes principais foi o que teve a maior quantidade de simulações com menor erro quadrático;

- nos cenários sem multicolinearidade, a quantidade de vezes que o estimador de máxima verossimilhança apresentou o menor erro quadrático sempre foi maior do que em relação à do estimador em componentes principais. Isso se deve ao fato de o estimador de componentes principais ser igual ao de máxima verossimilhança no caso em que o índice de condição da matriz de planejamento era menor do que dez e também pelo critério utilizado de se priorizar o estimador de máxima verossimilhança com o menor erro quadrático quando houvesse empates;

- quanto maior a quantidade de covariáveis, menor é a quantidade de vezes que o estimador de máxima verossimilhança apresenta o menor erro quadrático. 


\begin{tabular}{|c|c|c|c|c|c|}
\hline $\begin{array}{l}\text { Tam. da } \\
\text { amostra }\end{array}$ & $\begin{array}{l}\text { Quant. de } \\
\text { covariáveis }\end{array}$ & $\begin{array}{l}\text { Nível de } \\
\text { multicol. }\end{array}$ & $\begin{array}{c}\text { EMV } \\
\text { qtde }(\%)\end{array}$ & $\begin{array}{c}\text { Cristas } \\
\text { qtde }(\%)\end{array}$ & $\begin{array}{c}\mathrm{CP} \\
\text { qtde }(\%)\end{array}$ \\
\hline \multirow{12}{*}{100} & \multirow{3}{*}{5} & nenhum & $191(19 \%)$ & $739(74 \%)$ & $70(7 \%)$ \\
\hline & & moderado & $23(2 \%)$ & $960(96 \%)$ & $17(2 \%)$ \\
\hline & & severo 1 & $13(1 \%)$ & $397(40 \%)$ & $590(59 \%)$ \\
\hline & \multirow{4}{*}{10} & nenhum & $81(8 \%)$ & $903(90 \%)$ & $16(2 \%)$ \\
\hline & & moderado & $2(0 \%)$ & $883(88 \%)$ & $115(12 \%)$ \\
\hline & & severo 1 & $1(0 \%)$ & $648(65 \%)$ & $351(35 \%)$ \\
\hline & & severo 2 & $0(0 \%)$ & $353(35 \%)$ & $647(65 \%)$ \\
\hline & \multirow{5}{*}{15} & nenhum & $24(2 \%)$ & $971(97 \%)$ & $5(1 \%)$ \\
\hline & & moderado & $0(0 \%)$ & $797(80 \%)$ & $203(20 \%)$ \\
\hline & & severo 1 & $0(0 \%)$ & $623(62 \%)$ & $377(38 \%)$ \\
\hline & & severo 2 & $0(0 \%)$ & $475(48 \%)$ & $525(53 \%)$ \\
\hline & & severo 3 & $0(0 \%)$ & $347(35 \%)$ & $653(65 \%)$ \\
\hline \multirow{12}{*}{500} & \multirow{3}{*}{5} & nenhum & $257(26 \%)$ & $593(59 \%)$ & $150(15 \%)$ \\
\hline & & moderado & $61(6 \%)$ & $893(89 \%)$ & $46(5 \%)$ \\
\hline & & severo 1 & $46(5 \%)$ & $351(35 \%)$ & $603(60 \%)$ \\
\hline & \multirow{4}{*}{10} & nenhum & $188(19 \%)$ & $711(71 \%)$ & $101(10 \%)$ \\
\hline & & moderado & $24(2 \%)$ & $955(96 \%)$ & $21(2 \%)$ \\
\hline & & severo 1 & $13(1 \%)$ & $576(58 \%)$ & $411(41 \%)$ \\
\hline & & severo 2 & $3(0 \%)$ & $270(27 \%)$ & $727(73 \%)$ \\
\hline & \multirow{5}{*}{15} & nenhum & $112(11 \%)$ & $822(82 \%)$ & $66(7 \%)$ \\
\hline & & moderado & $3(0 \%)$ & $985(99 \%)$ & $12(1 \%)$ \\
\hline & & severo 1 & $3(0 \%)$ & $766(77 \%)$ & $231(23 \%)$ \\
\hline & & severo 2 & $1(0 \%)$ & $449(45 \%)$ & $550(55 \%)$ \\
\hline & & severo 3 & $0(0 \%)$ & $210(21 \%)$ & $790(79 \%)$ \\
\hline \multirow{12}{*}{1000} & \multirow{3}{*}{5} & nenhum & $243(24 \%)$ & $565(57 \%)$ & $192(19 \%)$ \\
\hline & & moderado & $86(9 \%)$ & $822(82 \%)$ & $92(9 \%)$ \\
\hline & & severo 1 & $78(8 \%)$ & $307(31 \%)$ & $615(62 \%)$ \\
\hline & \multirow{4}{*}{10} & nenhum & $202(20 \%)$ & $658(66 \%)$ & $140(14 \%)$ \\
\hline & & moderado & $47(5 \%)$ & $926(93 \%)$ & $27(3 \%)$ \\
\hline & & severo 1 & $28(3 \%)$ & $522(52 \%)$ & $450(45 \%)$ \\
\hline & & severo 2 & $1(0 \%)$ & $250(25 \%)$ & $749(75 \%)$ \\
\hline & \multirow{5}{*}{15} & nenhum & $164(16 \%)$ & $736(74 \%)$ & $100(10 \%)$ \\
\hline & & moderado & $11(1 \%)$ & $968(97 \%)$ & $21(2 \%)$ \\
\hline & & severo 1 & $15(2 \%)$ & $632(63 \%)$ & $353(35 \%)$ \\
\hline & & severo 2 & $3(0 \%)$ & $359(36 \%)$ & $638(64 \%)$ \\
\hline & & severo 3 & $0(0 \%)$ & $181(18 \%)$ & $819(82 \%)$ \\
\hline
\end{tabular}

Tabela 5.3: Quantidade e percentual de vezes em que cada estimador apresentou o menor erro quadrático médio. 
Por fim, no modelo de Regressão em Cristas, foi calculada a quantidade e o percentual de vezes em que cada parâmetro em cristas gerou estimativas com o menor erro quadrático médio. Essas quantidades encontram-se na Tabela 5.4. Considerando essa tabela podemos concluir que:

- em praticamente todos os cenários estudados, o estimador em cristas escolhido a maior quantidade de vezes, ou seja, o que apresentou o menor erro quadrático dentre os cinco parâmetros, foi $\lambda_{2}$. A única exceção foi para o cenário com nenhuma multicolinearidade, cinco covariáveis e tamanho de amostra igual a 1000, em que o parâmetro $\lambda_{5}$ foi escolhido em $45 \%$ das simulações. Neste cenário, o parâmetro $\lambda_{2}$ foi o segundo mais escolhido (em $42 \%$ das simulações);

- em geral, quanto maior o nível de multicolinearidade, quantidade de covariáveis e tamanho da amostra, maior era o percentual de vezes em que o parâmetro $\lambda_{2}$ se mostrava mais eficiente;

- o parâmetro $\lambda_{5}$ foi o segundo mais escolhido nos cenários com nenhuma ou moderada multicolinearidade. 


\begin{tabular}{|c|c|c|c|c|c|c|c|}
\hline $\begin{array}{l}\text { Tam. da } \\
\text { amostra }\end{array}$ & $\begin{array}{l}\text { Quant. de } \\
\text { covariáveis }\end{array}$ & $\begin{array}{l}\text { Nível de } \\
\text { multicol. }\end{array}$ & $\begin{array}{c}\lambda_{1} \\
\text { qtde }(\%)\end{array}$ & $\begin{array}{c}\lambda_{2} \\
\text { qtde }(\%)\end{array}$ & $\begin{array}{c}\lambda_{3} \\
\text { qtde }(\%)\end{array}$ & $\begin{array}{c}\lambda_{4} \\
\text { qtde }(\%)\end{array}$ & $\begin{array}{c}\lambda_{5} \\
\text { qtde }(\%)\end{array}$ \\
\hline \multirow{12}{*}{100} & \multirow{3}{*}{5} & nenhum & $66(7 \%)$ & $432(43 \%)$ & $124(12 \%)$ & $84(8 \%)$ & $294(29 \%)$ \\
\hline & & moderado & $45(5 \%)$ & $701(70 \%)$ & $113(11 \%)$ & $88(9 \%)$ & $53(5 \%)$ \\
\hline & & severo 1 & $70(7 \%)$ & $730(73 \%)$ & $87(9 \%)$ & $76(8 \%)$ & $37(4 \%)$ \\
\hline & \multirow{4}{*}{10} & nenhum & $53(5 \%)$ & $536(54 \%)$ & $198(20 \%)$ & $95(10 \%)$ & $118(12 \%)$ \\
\hline & & moderado & $12(1 \%)$ & $836(84 \%)$ & $95(10 \%)$ & $43(4 \%)$ & $14(1 \%)$ \\
\hline & & severo 1 & $9(1 \%)$ & $865(87 \%)$ & $68(7 \%)$ & $27(3 \%)$ & $31(3 \%)$ \\
\hline & & severo 2 & $5(1 \%)$ & $863(86 \%)$ & $53(5 \%)$ & $18(2 \%)$ & $61(6 \%)$ \\
\hline & \multirow{5}{*}{15} & nenhum & $29(3 \%)$ & $596(60 \%)$ & $224(22 \%)$ & $95(10 \%)$ & $56(6 \%)$ \\
\hline & & moderado & $0(0 \%)$ & $856(86 \%)$ & $76(8 \%)$ & $10(1 \%)$ & $58(6 \%)$ \\
\hline & & severo 1 & $2(0 \%)$ & $806(81 \%)$ & $46(5 \%)$ & $18(2 \%)$ & $128(13 \%)$ \\
\hline & & severo 2 & $1(0 \%)$ & $788(79 \%)$ & $26(3 \%)$ & $12(1 \%)$ & $173(17 \%)$ \\
\hline & & severo 3 & $0(0 \%)$ & $701(70 \%)$ & $13(1 \%)$ & $10(1 \%)$ & $276(28 \%)$ \\
\hline \multirow{12}{*}{500} & \multirow{3}{*}{5} & nenhum & $33(3 \%)$ & $421(42 \%)$ & $81(8 \%)$ & $48(5 \%)$ & $417(42 \%)$ \\
\hline & & moderado & $20(2 \%)$ & $733(73 \%)$ & $78(8 \%)$ & $55(6 \%)$ & $114(11 \%)$ \\
\hline & & severo 1 & $32(3 \%)$ & $745(75 \%)$ & $73(7 \%)$ & $92(9 \%)$ & $58(6 \%)$ \\
\hline & \multirow{4}{*}{10} & nenhum & $37(4 \%)$ & $462(46 \%)$ & $129(13 \%)$ & $72(7 \%)$ & $300(30 \%)$ \\
\hline & & moderado & $11(1 \%)$ & $840(84 \%)$ & $79(8 \%)$ & $22(2 \%)$ & $48(5 \%)$ \\
\hline & & severo 1 & $7(1 \%)$ & $869(87 \%)$ & $71(7 \%)$ & $38(4 \%)$ & $15(2 \%)$ \\
\hline & & severo 2 & $4(0 \%)$ & $918(92 \%)$ & $53(5 \%)$ & $20(2 \%)$ & $5(1 \%)$ \\
\hline & \multirow{5}{*}{15} & nenhum & $54(5 \%)$ & $447(45 \%)$ & $214(21 \%)$ & $96(10 \%)$ & $189(19 \%)$ \\
\hline & & moderado & $2(0 \%)$ & $888(89 \%)$ & $68(7 \%)$ & $27(3 \%)$ & $15(2 \%)$ \\
\hline & & severo 1 & $1(0 \%)$ & $907(91 \%)$ & $69(7 \%)$ & $20(2 \%)$ & $3(0 \%)$ \\
\hline & & severo 2 & $1(0 \%)$ & $944(94 \%)$ & $39(4 \%)$ & $15(2 \%)$ & $1(0 \%)$ \\
\hline & & severo 3 & $0(0 \%)$ & $969(97 \%)$ & $27(3 \%)$ & $4(0 \%)$ & $0(0 \%)$ \\
\hline \multirow{12}{*}{1000} & \multirow{3}{*}{5} & nenhum & $19(2 \%)$ & $424(42 \%)$ & $52(5 \%)$ & $59(6 \%)$ & $446(45 \%)$ \\
\hline & & moderado & $9(1 \%)$ & $714(71 \%)$ & $50(5 \%)$ & $46(5 \%)$ & $181(18 \%)$ \\
\hline & & severo 1 & $20(2 \%)$ & $734(73 \%)$ & $99(10 \%)$ & $64(6 \%)$ & $83(8 \%)$ \\
\hline & \multirow{4}{*}{10} & nenhum & $21(2 \%)$ & $481(48 \%)$ & $95(10 \%)$ & $49(5 \%)$ & $354(35 \%)$ \\
\hline & & moderado & $21(2 \%)$ & $801(80 \%)$ & $67(7 \%)$ & $34(3 \%)$ & $77(8 \%)$ \\
\hline & & severo 1 & $8(1 \%)$ & $894(89 \%)$ & $48(5 \%)$ & $18(2 \%)$ & $32(3 \%)$ \\
\hline & & severo 2 & $2(0 \%)$ & $943(94 \%)$ & $34(3 \%)$ & $19(2 \%)$ & $2(0 \%)$ \\
\hline & \multirow{5}{*}{15} & nenhum & $36(4 \%)$ & $494(49 \%)$ & $122(12 \%)$ & $71(7 \%)$ & $277(28 \%)$ \\
\hline & & moderado & $11(1 \%)$ & $850(85 \%)$ & $76(8 \%)$ & $26(3 \%)$ & $37(4 \%)$ \\
\hline & & severo 1 & $5(1 \%)$ & $914(91 \%)$ & $52(5 \%)$ & $13(1 \%)$ & $16(2 \%)$ \\
\hline & & severo 2 & $4(0 \%)$ & $956(96 \%)$ & $27(3 \%)$ & $9(1 \%)$ & $4(0 \%)$ \\
\hline & & severo 3 & $0(0 \%)$ & $984(98 \%)$ & $12(1 \%)$ & $4(0 \%)$ & $0(0 \%)$ \\
\hline
\end{tabular}

Tabela 5.4: Quantidade e percentual de vezes em que cada parâmetro em cristas gerou estimativas com o menor erro quadrático. 


\subsection{Considerações finais do capítulo}

A partir deste estudo de simulação pudemos concluir que os estimadores viesados apresentados anteriormente conseguiram diminuir drasticamente o erro quadrático médio em relação ao do estimador de máxima verossimilhança. Quanto menor o tamanho da amostra e maior o nível de multicolinearidade, maior foi o ganho em se utilizar esses estimadores. Além disso, pudemos notar também que o estimador em cristas conseguiu reduzir mais o erro quadrático médio nos cenários com multicolinearidade moderada e "severo 1" e nos cenários com multicolinearidade "severo 2" e "severo 3", o estimador em componentes principais foi o mais indicado. Dentre os parâmetros em cristas testados neste estudo de simulação o que apresentou o menor erro quadrático médio em praticamente todos os cenários foi $\lambda_{2}$.

No próximo capítulo, faremos uma aplicação de parte do conteúdo apresentado nos capítulos anteriores em um conjunto de dados reais. 


\section{Capítulo 6}

\section{Aplicação em um Conjunto de Dados Reais}

Neste capítulo, aplicaremos todas as técnicas abordadas anteriormente, desde a Análise de Regressão Logística, passando pelo diagnóstico da multicolinearidade até a utilização dos estimadores viesados, a um conjunto de dados reais extraídos de Singer (2012). Estes dados fazem parte da publicação da Professora Doutora Valeria Bezerra de Carvalho, da Instituição Interestadual de Cardiologia (INTERCOR), e foram analisados em Singer e Ikeda (1996).

\subsection{Descrição do estudo}

O estudo envolveu 1500 pacientes que procuraram uma clínica particular de cardiologia com alguma queixa entre 1989 e 1994 e foram avaliados transversalmente do ponto de vista clínico, bioquímico e cineangiocoronariográfico. Um dos objetivos era investigar a associação entre os fatores de risco e o grau de lesão obstrutiva (LO) das artérias coronarianas. A variável resposta foi construída segundo o seguinte critério:

$Y_{i}= \begin{cases}1, & \text { se o i-ésimo paciente possui LO coronariana } \geq 50 \% \text { em pelo menos uma de três } \\ & \text { artérias } \\ 0, & \text { caso contrário }\end{cases}$

As artérias observadas para a classificação da presença de lesão foram: artéria coronária direita, artéria descendente anterior e artéria circunflexa.

O banco de dados é constituído de 68 variáveis, porém somente 37 delas eram de interesse para o estudo, sendo que uma delas é a variável resposta. A descrição dessas covariáveis pode ser vista a seguir, de acordo com o seu tipo:

- Clínicas: gênero, idade para os pacientes do gênero feminino e idade para os pacientes do gênero masculino e presença de manifestações clínicas, tais como angina estável, 
angina instável, insuficiência cardíaca, arritmia, infarto do miocárdio, hipertensão arterial sistêmica (pressão arterial sistólica maior ou igual a $140 \mathrm{mmHg}$ ou diastólica maior ou igual a $90 \mathrm{mmHg}$ ou uso de drogas antihipertensivas), diabete mellitus (valor da glicemia de jejum maior que $120 \mathrm{mg} / \mathrm{dl}$ ou uso de drogas hipoglicemiantes), tabagismo, obesidade (índice de massa corpórea maior ou igual a $33 \mathrm{~kg} / \mathrm{m}^{2}$ para indivíduos do sexo masculino ou maior ou igual a $30 \mathrm{~kg} / \mathrm{m}^{2}$ para indivíduos do sexo feminino) e presença de antecedentes hereditários de doença arterial coronariana;

- Bioquímicas e hematológicas: níveis plasmáticos $(\mathrm{mg} / \mathrm{dl})$ de colesterol total (CT), lipoproteínas de baixa (LDL), alta (HDL) e muito baixa densidade (VLDL), os quocientes CT/HDL e LDL/HDL, triglicérides, glicemia de jejum, fibrinogênio ( $\mathrm{mm} / \%)$ e plaquetas $\left(1000 / \mathrm{mm}^{3}\right)$. Como os níveis de colesterol total e suas frações (LDL, HDL e VLDL), além de triglicérides e glicose podem ser influenciados pelo uso de remédios, em cada caso, foram criadas interações dessas covariáveis com o uso e não uso de medicamento;

- Angiográficas: presença de LO coronárias expressivas em pelo menos uma das 3 artérias, extensão do comprometimento pela doença em uma, duas ou três artérias, de acordo com o número de artérias com LO maior ou igual a $50 \%$ e evidências de doença arterial carotídea ou periférica ao exame físico, estudo arteriográfico ou ultrassonográfico.

A descrição de cada covariável, assim como o significado de cada categoria, pode ser encontrado nas Tabelas D.1 e D.2 no Apêndice D. Neste mesmo apêndice pode ser observado nas Tabelas D.3 à D.18 que das 37 covariáveis de interesse, 14 apresentaram uma quantidade muito pequena de preenchimento, ou seja, menos do que 1100 pacientes. Assim, essas covariáveis foram desconsideradas da análise. Além disso, das 23 covariáveis restantes, 11 não apresentaram associação com a variável resposta univariadamente a um nível de significância de 0,0001, de acordo com as Tabelas D.19 à D.23. Foi utilizado esse nível de significância devido à grande quantidade de indivíduos do estudo.

Observou-se então que o conjunto de covariáveis que auxilia na previsão da variável de interesse é: DIAB, TABAG, ANGEST, ANGINS, ARRIT, GENERO, INFARTO, COLM, IDADFEM, IDADMAS, TRIGM e GLICM. O modelo proposto encontra-se a seguir:

$$
\pi\left(\mathbf{x}_{i}\right)=\frac{\exp \left(\beta_{0}+\beta_{1} x_{i 1}+\beta_{2} x_{i 2}+\ldots+\beta_{10} x_{i 11}+\beta_{11} x_{i 12}\right)}{1+\exp \left(\beta_{0}+\beta_{1} x_{i 1}+\beta_{2} x_{i 2}+\ldots+\beta_{11} x_{i 11}+\beta_{12} x_{i 12}\right)}, i=1,2, \ldots, 1500
$$

em que:

- $x_{i 1}$ é igual a um se o i-ésimo paciente apresentar diabetes mellitos e é igual a zero caso contrário. É referente à covariável DIAB; 
- $x_{i 2}$ é igual a um se o i-ésimo paciente fuma e é igual a zero caso contrário. É referente à covariável TABAG;

- $x_{i 3}$ é igual a um se o i-ésimo paciente apresentar angina estável e é igual a zero caso contrário. É referente à covariável ANGEST;

- $x_{i 4}$ é igual a um se o i-ésimo paciente apresentar angina instável e é igual a zero caso contrário. É referente à covariável ANGINS;

- $x_{i 5}$ é igual a um se o i-ésimo paciente apresentar arritmia e é igual a zero caso contrário. É referente à covariável ARRIT;

- $x_{i 6}$ é igual a um se o i-ésimo paciente for do gênero feminino e é igual a zero se for do gênero masculino. É referente à covariável GENERO;

- $x_{i 7}$ é igual a um se o i-ésimo paciente já tiver apresentado infarto e é igual a zero caso contrário. É referente à covariável INFARTO;

- $x_{i 8}$ é o valor padronizado do colesterol do i-ésimo paciente se ele utilizar medicamento e é igual a zero caso se não utilizar. É referente à covariável COLM;

- $x_{i 9}$ é a idade padronizada do i-ésimo paciente se ele for do gênero feminino e é igual a zero caso contrário. É referente à covariável IDADFEM;

- $x_{i 10}$ é a idade padronizada do i-ésimo paciente se ele for do gênero masculino e é igual a zero caso contrário. É referente à covariável IDADMAS;

- $x_{i 11}$ é o valor padronizado do triglicérides do i-ésimo paciente se ele utilizar medicamento e é igual a zero caso contrário. É referente à covariável TRIGM;

- $x_{i 12}$ é o valor padronizado da glicose do i-ésimo paciente se ele utilizar medicamento e é igual a zero caso contrário. É referente à covariável GLICM.

As estimativas de máxima verossimilhança dos coeficientes referentes às covariáveis do modelo dado por (6.1) podem ser encontrados na Tabela 6.1. Já, a Tabela D.27 do Apêndice D apresenta as estimativas das variâncias e covariâncias dos estimadores dos parâmetros deste modelo. Todas as análises realizadas neste capítulo foram feitas com o auxílio do software $\mathrm{R}$ versão 2.14 .2 e o código completo pode ser visto no Apêndice C. 


\begin{tabular}{lcc}
\hline \hline & Covariável & EMV \\
\hline$\hat{\beta}_{0}$ & Constante & $-0,50(0,87)$ \\
$\hat{\beta}_{1}$ & DIAB & $-0,74(0,63)$ \\
$\hat{\beta}_{2}$ & TABAG & $0,02(0,18)$ \\
$\hat{\beta}_{3}$ & ANGEST & $0,65(0,19)^{* *}$ \\
$\hat{\beta}_{4}$ & ANGINS & $1,46(0,20)^{* * *}$ \\
$\hat{\beta}_{5}$ & ARRIT & $-0,66(0,28)$ \\
$\hat{\beta}_{6}$ & GENERO & $0,49(1,11)$ \\
$\hat{\beta}_{7}$ & INFARTO & $2,55(0,25)^{* * *}$ \\
$\hat{\beta}_{8}$ & COLM & $-0,14(0,22)$ \\
$\hat{\beta}_{9}$ & IDADFEM & $1,30(0,42)^{* *}$ \\
$\hat{\beta}_{10}$ & IDADMAS & $1,60(0,25)^{* * *}$ \\
$\hat{\beta}_{11}$ & TRIGM & $0,63(0,28)$. \\
$\hat{\beta}_{12}$ & GLICM & $0,60(0,28)$. \\
\hline Num. obs. & 1025 & \\
\hline \multirow{2}{***}{$p<=0,0001,{ }^{* *} p<=0,001,{ }^{*}$} & $p<=0,01, \cdot p<=0,05$ \\
\hline \hline
\end{tabular}

Tabela 6.1: Estimativas dos parâmetros e dos erros padrão dos estimadores dos parâmetros do modelo de regressão logística com todas as covariáveis significantes univariadamente a um nivel de 0,0001 .

Podemos perceber que, apesar de todas as covariáveis presentes no modelo serem significativas univariadamente a um nível de significância de 0,0001, as covariáveis referente à DIAB, TABAG, ANGEST, ARRIT, GENERO, COLM, IDADFEM, TRIGM e GLICM não se mostraram significantes a um nível de 0,0001 no modelo multivariado. Isso pode ser um grande indício de presença de multicolinearidade nos dados, pois, conforme foi visto nas Subseções 3.1.2 e 3.1.4 do Capítulo 3, as estimativas de máxima verossimilhança dos parâmetros, das suas variâncias e as conclusões dos testes de significância dos parâmetros podem estar seriamente comprometidos nessas circunstâncias. Vamos, então, analisar se neste conjunto de covariáveis a multicolinearidade está presente.

\subsection{Identificação da multicolinearidade}

De acordo com a Tabela 6.1, podemos perceber que, dos 1500 pacientes presentes na amostra, somente 1025 apresentaram todas as covariáveis selecionadas preenchidas. Daqui para frente, portanto, vamos analisar somente esses pacientes.

O primeiro índice para identificação da multicolinearidade será o coeficiente de determinação da regressão linear das covariáveis em relação às demais, conforme foi visto em (3.12). Para obter esse índice é necessário ajustar um modelo de regressão linear de cada covariável 
em função das demais. Assim, na Tabela 6.2 foram sumarizados os valores de $R_{j}^{2}$ que podem ser encontrados em maiores detalhes nas Tabelas D.24 a D.26 no Apêndice D.

\begin{tabular}{cccccc}
\hline \hline$R_{1}^{2}$ & $R_{2}^{2}$ & $R_{3}^{2}$ & $R_{4}^{2}$ & $R_{5}^{2}$ & $R_{6}^{2}$ \\
DIAB & TABAG & ANGEST & ANGINS & ARRIT & GENERO \\
\hline $83,0 \%$ & $14,9 \%$ & $18,0 \%$ & $12,4 \%$ & $2,9 \%$ & $97,3 \%$ \\
\hline \hline$R_{7}^{2}$ & $R_{8}^{2}$ & $R_{9}^{2}$ & $R_{10}^{2}$ & $R_{11}^{2}$ & $R_{12}^{2}$ \\
INFARTO & COLM & IDADFEM & IDADMAS & TRIGM & GLICM \\
\hline $9,7 \%$ & $79,1 \%$ & $96,6 \%$ & $88,3 \%$ & $79,3 \%$ & $82,9 \%$ \\
\hline \hline
\end{tabular}

Tabela 6.2: Coeficiente de determinação da regressão linear das covariáveis em relação às demais.

De acordo com a Tabela 3.3, temos que a multicolinearidade severa é representada por coeficientes de determinação maiores do que 95\%. Assim, de acordo com esse critério, somente as covariáveis GENERO e IDADFEM estão envolvidas em multicolinearidades severas. Os coeficientes de determinação das covariáveis DIAB, IDADMAS, COLM, TRIGM e GLICM, apesar de estarem dentro do limite de multicolinearidade fraca, estão próximos ao limite superior, portanto, uma atenção maior deve ser dada à elas.

Depois de calculado o $R_{j}^{2}$, devemos fazer a decomposição em valores singulares da matriz $\hat{\mathbf{K}}$, para obter o número e o índice de condição e para fazer a decomposição da variância. A decomposição em valores singulares da matriz de planejamento pode ser encontrada na Tabela 6.3. Cada coluna da Tabela 6.3 , ou seja, $v_{0}, v_{1}, \ldots, v_{12}$, é um autovetor da matriz de planejamento e cada componente presente na última linha, $\mu_{0}, \mu_{1}, \ldots, \mu_{12}$, é um valor singular associado à cada autovetor. 


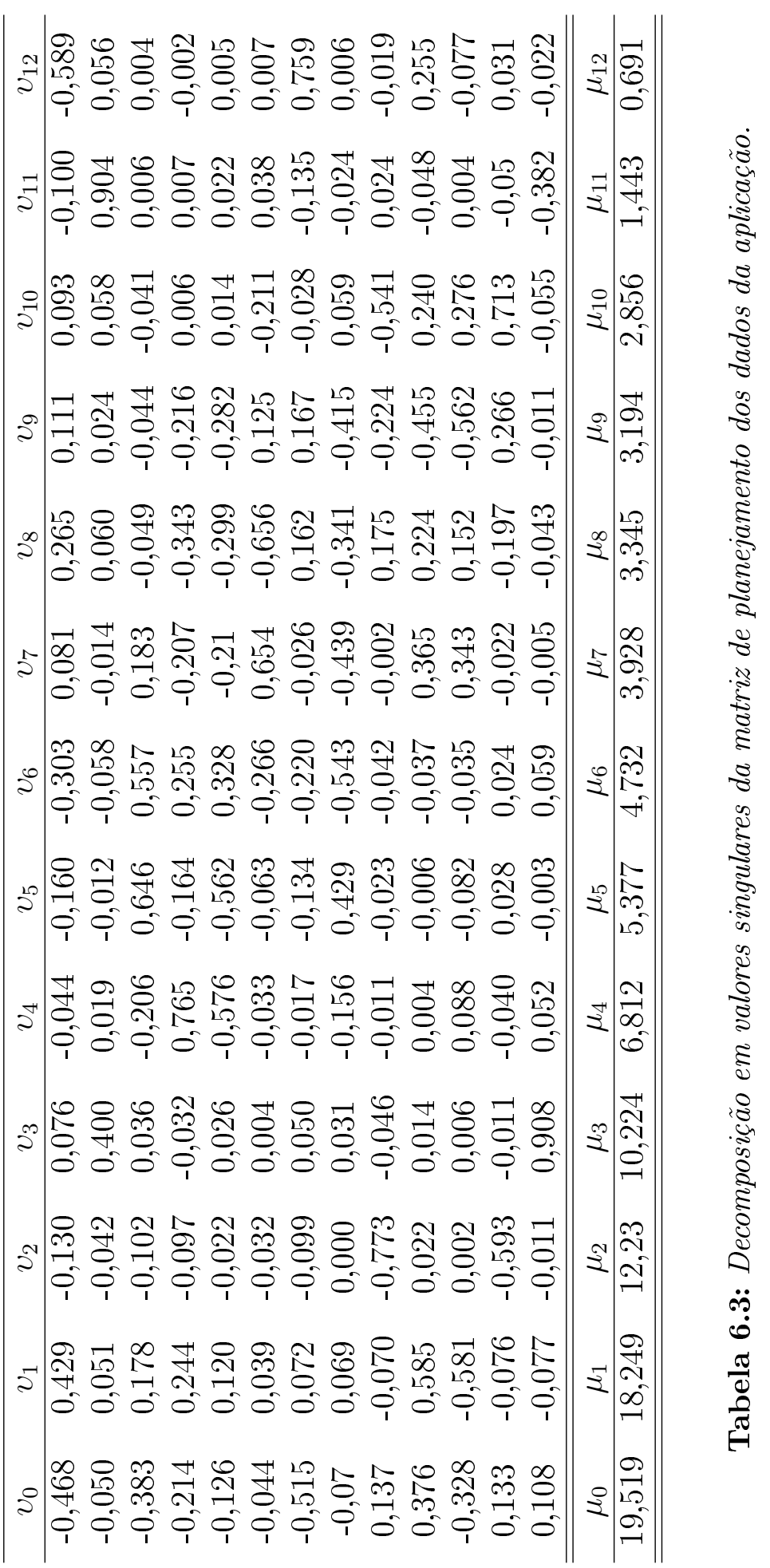


Os índices de condição da matriz de planejamento, como foi visto em (3.13), são iguais à divisão do seu maior autovalor por todos os demais. Eles podem ser encontrados na Tabela 6.4 .

\begin{tabular}{ccccccc}
\hline \hline$\eta_{0}$ & $\eta_{1}$ & $\eta_{2}$ & $\eta_{3}$ & $\eta_{4}$ & $\eta_{5}$ & $\eta_{6}$ \\
\hline 1,000 & 1,070 & 1,596 & 1,909 & 2,865 & 3,630 & 4,125 \\
\hline \hline$\eta_{7}$ & $\eta_{8}$ & $\eta_{9}$ & $\eta_{10}$ & $\eta_{11}$ & $\eta_{12}$ & \\
\hline 4,969 & 5,835 & 6,111 & 6,834 & 13,527 & 28,247 & \\
\hline \hline
\end{tabular}

Tabela 6.4: Índices de condição da matriz de planejamento dos dados da aplicação.

Como índices de condição acima de 10 já representam multicolinearidade mais do que fracas, concluímos que a matriz de planejamento apresenta duas relações de multicolinearidade moderadas. Além disso, também foi visto em (3.14), que o índice de condição de $\hat{\mathbf{K}}$ será o maior de todos os índices de condição, ou seja, será dado por:

$$
\eta=\eta_{12}=\frac{19,519}{0,691}=28,247 .
$$

Já os números de condição de $\hat{\mathbf{K}}^{\prime} \hat{\mathbf{K}}$, conforme visto em (3.15), podem ser encontrados na Tabela 6.5.

\begin{tabular}{ccccccc}
\hline \hline$\eta_{0}^{*}$ & $\eta_{1}^{*}$ & $\eta_{2}^{*}$ & $\eta_{3}^{*}$ & $\eta_{4}^{*}$ & $\eta_{5}^{*}$ & $\eta_{6}^{*}$ \\
\hline 1,000 & 1,144 & 2,547 & 3,645 & 8,211 & 13,179 & 17,017 \\
\hline \hline$\eta_{7}^{*}$ & $\eta_{8}^{*}$ & $\eta_{9}^{*}$ & $\eta_{10}^{*}$ & $\eta_{11}^{*}$ & $\eta_{12}^{*}$ & \\
\hline 24,691 & 34,05 & 37,354 & 46,715 & 182,943 & 798,899 & \\
\hline \hline
\end{tabular}

Tabela 6.5: Números de condição da matriz de planejamento dos dados da aplicação.

Analisando a Tabela 6.5, percebemos que dois dos valores dos números de condição são maiores do que 100, ou seja, existem duas relações de multicolinearidade moderadas, o que vai de encontro com a conclusão obtida através do índice de condição.

Por fim, vamos fazer a decomposição da variância dos estimadores de máxima verossimilhança, de acordo com o que foi visto em (3.16), para identificar quais covariáveis estão envolvidas nas multicolinearidades. A Tabela 6.6 apresenta os valores de $\omega_{k j}$, a proporção da decomposição da variância de $\hat{\beta}_{k}$ associado ao j-ésimo componente dessa decomposição, $j, k=0, \ldots, 12$. 


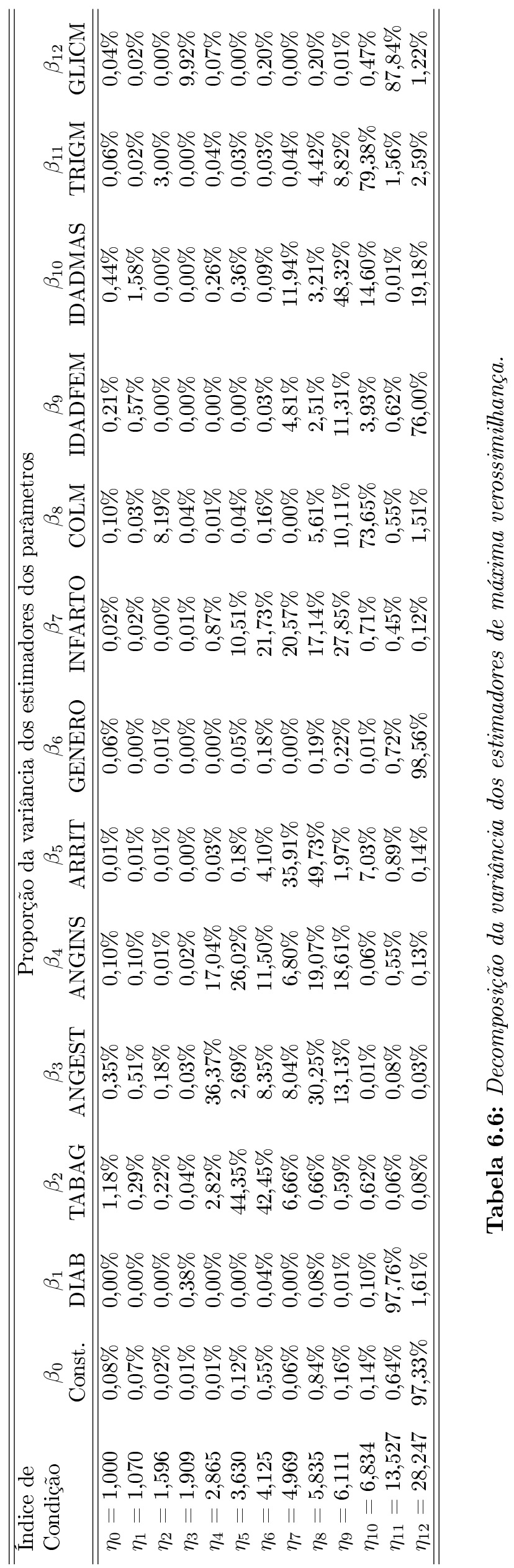


Analisando a primeira coluna da Tabela 6.6, por exemplo, podemos concluir que a constante do modelo está envolvida em uma multicolinearidade. O mesmo pode ser concluído para as covariáveis DIAB, GENERO,COLM, IDADFEM, TRIGM e GLICM, representadas na segunda, sétima, nona, décima, décima segunda e décima terceira coluna, respectivamente. Já, analisando as linhas da Tabela 6.6, temos que a multicolinearidade mais forte, relacionada com $\eta_{12}$ e representada na última linha da tabela, envolve a constante $\left(\beta_{0}\right)$ e as covariáveis GENERO $\left(\beta_{6}\right)$ e IDADFEM $\left(\beta_{9}\right)$. Essa relação faz bastante sentido, já que a covariável IDADFEM foi construída como uma interação entre GENERO e a idade do paciente. Analisando a penúltima linha da tabela concluímos que a segunda relação de multicolinearidade mais forte envolve as covariáveis DIAB $\left(\beta_{1}\right)$ e $\operatorname{GLICM}\left(\beta_{12}\right)$. Essa associação também é bem intuitiva, pois a covariável GLICM foi construída como uma interação entre a taxa de glicemia do paciente e a covariável DIAB. As covariáveis COLM e TRIGM estão relacionadas com $\eta_{10}$, porém este índice de condição não está relacionado com a multicolinearidade.

As conclusões a que chegamos baseadas nas quatro medidas utilizadas na identificação da multicolinearidade, portanto, são que, dentre as covariáveis presentes neste banco de dados:

- existem duas relações de multicolinearidade moderadas, de acordo com os índices e números de condição;

- através da análise do coeficiente de determinação e da decomposição da variância, temos que as covariáveis GENERO, IDADFEM, DIAB e GLICM estão envolvidas nas multicolinearidades moderadas.

\subsection{Aplicação dos estimadores viesados}

Identificadas as relações de multicolinearidade, vamos agora aplicar os dois métodos vistos no Capítulo 4: Regressão em Cristas e Regressão em Componentes Principais. Ao final desta seção, estes dois estimadores serão comparados afim de identificar qual apresentou a maior redução da estimativa do erro quadrático médio.

\subsubsection{Aplicação da Regressão em Cristas}

Conforme foi visto em (4.13), o estimador em cristas é função do parâmetro $\lambda$. Portanto, vamos, primeiramente, tentar identificar o "melhor" valor para esse parâmetro através da análise do traço de Ridge, presente na Figura 6.1. Neste gráfico, podemos encontrar as estimativas para os parâmetros do modelo de acordo com o parâmetro $\lambda$. O objetivo aqui é tentar encontrar o menor valor de $\lambda$ que faz com que a estimativa de todos os parâmetros do modelo fique com o sinal correto e estável.

Analisando a Figura 6.1, temos que as estimativas de máxima verossimilhança dos parâmetros que estavam com os sinais invertidos estão destacadas em preto e as estimativas 


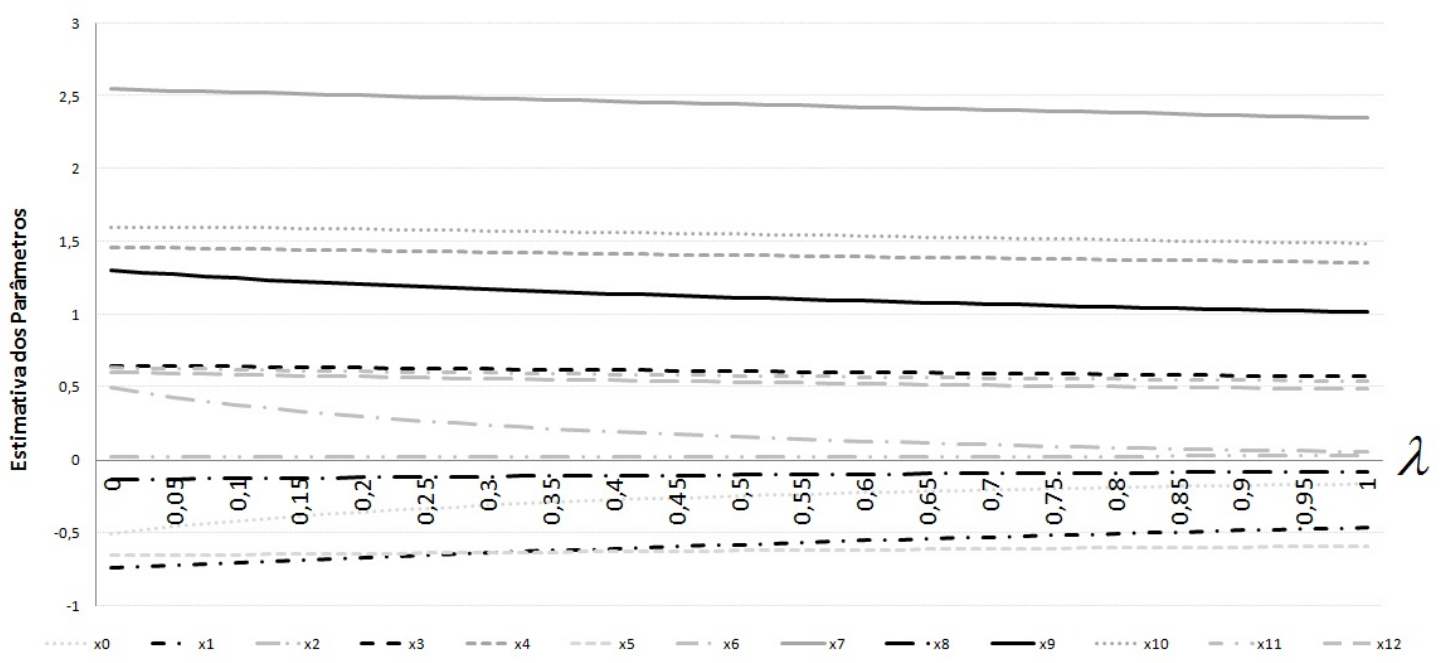

Figura 6.1: Traço de Ridge com as estimativas dos parâmetros do modelo referente aos dados de aplicação.

dos demais parâmetros estão em cinza. Além disso, temos que dentro do intervalo [0;1] não existe nenhum valor de $\lambda$ que corrige o sinal de todas as estimativas dos parâmetros do modelo. Dessa forma, vamos calcular os valores para este parâmetro de acordo com as fórmulas dadas por (4.17) a (4.21). Esses valores podem ser encontrados na Tabela 6.7.

\begin{tabular}{ccccc}
\hline \hline$\lambda_{1}$ & $\lambda_{2}$ & $\lambda_{3}$ & $\lambda_{4}$ & $\lambda_{5}$ \\
\hline 0,102 & 0,835 & 0,552 & 0,168 & 0,019 \\
\hline \hline
\end{tabular}

Tabela 6.7: Valores para o parâmetro em cristas.

Utilizando os valores de $\lambda_{k}$ descritos na Tabela 6.7 , podemos calcular as estimativas dos parâmetros referentes às covariáveis do modelo de Regressão em Cristas. O modelo será igual ao apresentado em (6.1), porém a forma de estimação dos parâmetros será a descrita em (4.13). Na Tabela 6.8 podem ser encontradas as estimativas dos parâmetros de acordo com o valor de $\lambda_{k}$ utilizado, bem como as estimativas dos seus erros padrão. As matrizes com as estimativas das variâncias dos estimadores dos parâmetros desses modelos podem ser encontradas nas Tabelas D.28 a D.32 do Apêndice D.

Para compararmos a qualidade do ajuste dos modelos, calculamos a estatística do Teste de Hosmer-Lemeshow, como dado em (2.14). Utilizamos como ponto de corte de cada grupo o valor dos decis da probabilidade estimada do evento de interesse. As tabelas auxiliares para o cálculo dessa estatística podem ser encontradas na Tabela 6.9 e os valores das estatísticas, assim como os seus respectivos p-valor e a variação do valor da estatística do teste de ajuste desses modelos em relação ao ajuste de máxima verossimilhança, podem ser encontrados na Tabela 6.10 . 


\begin{tabular}{lcccccc}
\hline \hline & Covariável & $\lambda_{1}$ & $\lambda_{2}$ & $\lambda_{3}$ & $\lambda_{4}$ & $\lambda_{5}$ \\
\hline$\hat{\beta}_{0}$ & Constante & $-0,42(0,72)$ & $-0,18(0,34)$ & $-0,23(0,42)$ & $-0,37(0,65)$ & $-0,49(0,83)$ \\
$\hat{\beta}_{1}$ & DIAB & $-0,70(0,60)$ & $-0,50(0,45)$ & $-0,57(0,50)$ & $-0,68(0,59)$ & $-0,73(0,63)$ \\
$\hat{\beta}_{2}$ & TABAG & $0,02(0,18)$ & $0,03(0,17)$ & $0,02(0,18)$ & $0,02(0,18)$ & $0,02(0,18)$ \\
$\hat{\beta}_{3}$ & ANGEST & $0,64(0,19)$ & $0,58(0,18)$ & $0,61(0,18)$ & $0,64(0,18)$ & $0,65(0,19)$ \\
$\hat{\beta}_{4}$ & ANGINS & $1,45(0,20)$ & $1,37(0,20)$ & $1,40(0,20)$ & $1,44(0,20)$ & $1,46(0,20)$ \\
$\hat{\beta}_{5}$ & ARRIT & $-0,65(0,28)$ & $-0,60(0,26)$ & $-0,62(0,27)$ & $-0,65(0,27)$ & $-0,66(0,28)$ \\
$\hat{\beta}_{6}$ & GENERO & $0,38(0,92)$ & $0,08(0,42)$ & $0,14(0,52)$ & $0,32(0,82)$ & $0,47(1,07)$ \\
$\hat{\beta}_{7}$ & INFARTO & $2,53(0,24)$ & $2,38(0,23)$ & $2,43(0,24)$ & $2,51(0,24)$ & $2,54(0,25)$ \\
$\hat{\beta}_{8}$ & COLM & $-0,13(0,22)$ & $-0,09(0,20)$ & $-0,10(0,21)$ & $-0,12(0,22)$ & $-0,13(0,22)$ \\
$\hat{\beta}_{9}$ & IDADFEM & $1,25(0,37)$ & $1,04(0,23)$ & $1,10(0,26)$ & $1,22(0,34)$ & $1,29(0,41)$ \\
$\hat{\beta}_{10}$ & IDADMAS & $1,60(0,24)$ & $1,51(0,21)$ & $1,55(0,22)$ & $1,59(0,24)$ & $1,6(0,25)$ \\
$\hat{\beta}_{11}$ & TRIGM & $0,62(0,28)$ & $0,55(0,25)$ & $0,57(0,26)$ & $0,61(0,27)$ & $0,63(0,28)$ \\
$\hat{\beta}_{12}$ & GLICM & $0,59(0,27)$ & $0,50(0,21)$ & $0,53(0,23)$ & $0,58(0,26)$ & $0,60(0,28)$ \\
\hline \hline
\end{tabular}

Tabela 6.8: Estimativas dos parâmetros e dos erros padrão dos estimadores dos parâmetros dos modelos de Regressão em Cristas, segundo o valor do parâmetro em cristas. 


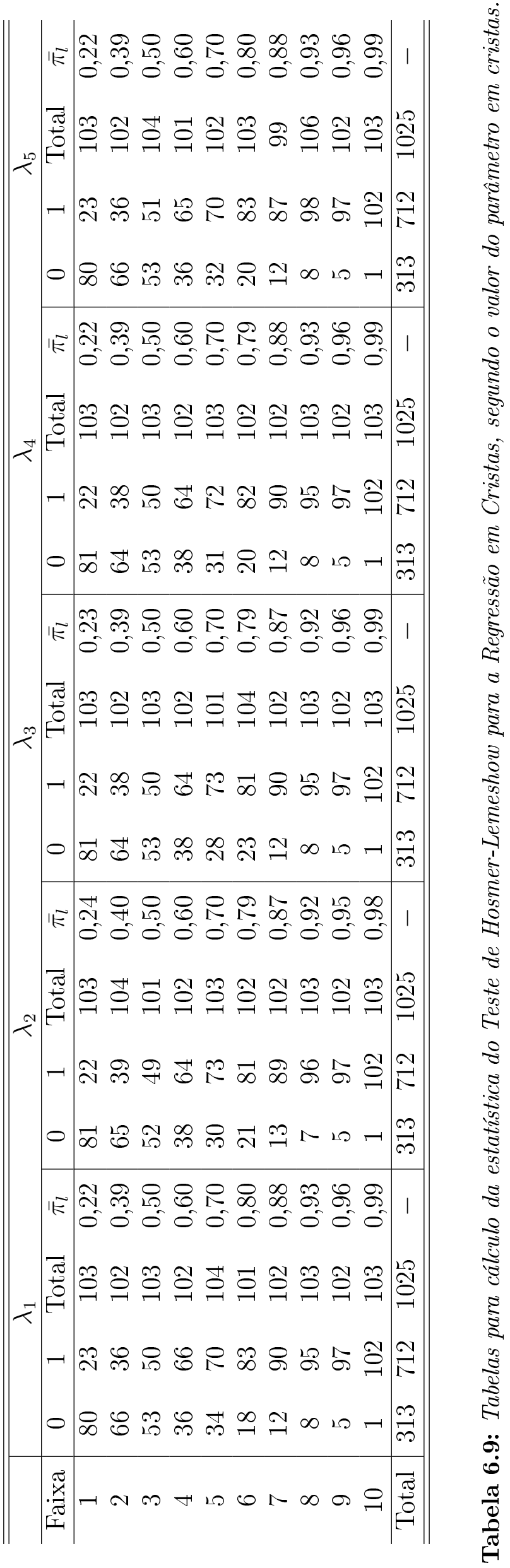




\begin{tabular}{lcccccc}
\hline \hline & EMV & $\lambda_{1}$ & $\lambda_{2}$ & $\lambda_{3}$ & $\lambda_{4}$ & $\lambda_{5}$ \\
\hline$\hat{C}$ & 2,970 & 2,928 & 1,623 & 1,643 & 1,13 & 2,041 \\
p-valor & - & 0,939 & 0,990 & 0,990 & 0,997 & 0,980 \\
Variação & - & $-1,4 \%$ & $-45,3 \%$ & $-44,7 \%$ & $-61,9 \%$ & $-31,3 \%$ \\
\hline \hline
\end{tabular}

Tabela 6.10: Valores das estatísticas e dos p-valores do Teste de Hosmer-Lemeshow e a variação da estatística dos modelos de Regressão em Cristas em relação ao de máxima verossimilhança, segundo o valor do parâmetro em cristas.

Analisando a Tabela 6.10 temos que $\lambda_{4}$ foi o valor de $\lambda$ que apresentou o menor valor da estatística do Teste de Hosmer-Lemeshow e, consequentemente, o maior p-valor e a maior redução do valor dessa estatística em relação ao ajuste de máxima verossimilhança, portanto, foi o modelo que apresentou o melhor desempenho dentre os cinco apresentados.

\subsubsection{Aplicação da Regressão em Componentes Principais}

Conforme já foi visto anteriormente, existem, dentre as covariáveis do modelo, duas relações de multicolinearidade moderadas. Dessa forma, para obter as estimativas dos parâmetros do modelo de regressão em Componentes Principais, como em (4.27), deveremos considerar somente os 11 primeiros autovetores resultantes da decomposição em valores singulares da matriz de planejamento. Assim, na Tabela 6.11, podem ser encontradas as estimativas dos parâmetros do modelo, as suas respectivas estimativas dos erros padrão e o p-valor associado à estatística de Wald. Já, a matriz com as estimativas das variâncias desse modelo pode ser encontrada na Tabela D.33 do Apêndice D.

\begin{tabular}{lcc}
\hline \hline & Covariável & CP \\
\hline$\hat{\beta}_{0}$ & Constante & $-0,10(0,12)$ \\
$\hat{\beta}_{1}$ & DIAB & $0,17(0,05)$ \\
$\hat{\beta}_{2}$ & TABAG & $0,02(0,18)$ \\
$\hat{\beta}_{3}$ & ANGEST & $0,66(0,19)$ \\
$\hat{\beta}_{4}$ & ANGINS & $1,48(0,20)$ \\
$\hat{\beta}_{5}$ & ARRIT & $-0,62(0,28)$ \\
$\hat{\beta}_{6}$ & GENERO & $-0,31(0,09)$ \\
$\hat{\beta}_{7}$ & INFARTO & $2,52(0,25)$ \\
$\hat{\beta}_{8}$ & COLM & $-0,09(0,22)$ \\
$\hat{\beta}_{9}$ & IDADFEM & $1,03(0,20)$ \\
$\hat{\beta}_{10}$ & IDADMAS & $1,67(0,23)$ \\
$\hat{\beta}_{11}$ & TRIGM & $0,55(0,27)$ \\
$\hat{\beta}_{12}$ & GLICM & $0,22(0,09)$ \\
\hline \hline
\end{tabular}

Tabela 6.11: Estimativas dos parâmetros e dos erros padrão dos estimadores dos parâmetros do modelo de Regressão em Componentes Principais.

Para compararmos a qualidade do ajuste do modelo, também foi calculado o Teste de 
Hosmer-Lemeshow, utilizando como ponto de corte de cada grupo o valor dos decis da probabilidade estimada do evento de interesse. A tabela auxiliar para o cálculo dessa estatística pode ser encontrada na Tabela 6.12. O valor da estatística, assim como o respectivo p-valor e a variação do valor da estatística do teste de ajuste desse modelo em relação ao ajuste de máxima verossimilhança, encontram-se na Tabela 6.13.

\begin{tabular}{c|cccc}
\hline \hline & \multicolumn{4}{|c}{ CP } \\
\hline Faixa & 0 & 1 & Total & $\bar{\pi}_{l}$ \\
\hline 1 & 82 & 20 & 102 & 0,221 \\
2 & 62 & 41 & 103 & 0,382 \\
3 & 55 & 48 & 103 & 0,496 \\
4 & 36 & 66 & 102 & 0,597 \\
5 & 30 & 74 & 104 & 0,703 \\
6 & 21 & 80 & 101 & 0,795 \\
7 & 14 & 87 & 101 & 0,873 \\
8 & 7 & 97 & 104 & 0,926 \\
9 & 5 & 97 & 102 & 0,960 \\
10 & 1 & 102 & 103 & 0,986 \\
\hline Total & 313 & 712 & 1025 & - \\
\hline \hline
\end{tabular}

Tabela 6.12: Tabelas para cálculo do Teste de Hosmer-Lemeshow para a Regressão em Componentes Principais.

\begin{tabular}{lcc}
\hline \hline & EMV & CP \\
\hline$\hat{C}$ & 2,970 & 2,497 \\
p-valor & - & 0,962 \\
Variação & - & $-15,9 \%$ \\
\hline \hline
\end{tabular}

Tabela 6.13: Valor da estatística e do p-valor do Teste de Hosmer-Lemeshow e a variação da estatística do modelo de Regressão em Componentes Principais em relação ao ajuste de máxima verossimilhança.

Analisando a Tabela 6.13 temos que o estimador em Componentes Principais conseguiu reduzir o valor da estatística de Hosmer-Lemeshow em relação ao de máxima verossimilhança, portanto, tivemos um ganho no ajuste deste modelo.

\subsection{Considerações finais do capítulo}

Conforme foi visto nas subsessões anteriores, pudemos concluir que tanto o modelo de Regressão em Cristas como o modelo de Regressão em Componentes Principais conseguiu ajustar melhor a variável de interesse em relação ao modelo de regressão logística usual. Dentre as duas técnicas, a que apresentou a maior redução do valor da estatística de Hosmer-Lemeshow em relação ao valor dessa estatística do modelo utilizando máxima verossimilhança foi o mo- 
delo de Regressão em Cristas, adotando o valor do parâmetro em cristas igual a 0,168 $\left(\lambda_{4}\right)$, conseguindo reduzir em $61,9 \%$ o valor dessa estatística.

Além disso, comparando os resultados obtidos analisando esse conjunto de dados reais com os obtidos no estudo de simulação, temos que o cenário mais semelhante aos dados utilizados nesta aplicação foi o que apresentou $n=1000, j=10$ e nível de multicolinearidade "severo 3", que forneceu o valor de $\eta$ mais próximo ao da aplicação, em média. Assim, notamos que as conclusões foram semelhantes no sentido de que os dois estimadores viesados conseguiram ajustar melhor aos dados em relação ao estimador de máxima verossimilhança. A única divergência está no fato de que o estimador que mais reduziu o erro quadrático médio no estudo de simulação dentro do cenário especificado anteriormente foi o estimador em componentes principais e nesta aplicação o estimador que mais reduziu a estatística de Hosmer-Lemeshow foi o estimador em cristas. Provavelmente isso ocorreu devido ao fato que as medidas de qualidade de ajuste utilizadas no estudo de simulação e nesta aplicação não foram as mesmas. 


\section{Capítulo 7}

\section{Considerações finais e sugestões para pesquisas futuras}

Nessa dissertação dois estimadores para os parâmetros do modelo de regressão logística foram apresentados como alternativas para o estimador de máxima verossimilhança no caso em que os dados são multicolineares. Inicialmente o modelo de regressão logística foi apresentado e, em seguida, definimos a multicolinearidade e expusemos os seus efeitos em modelos de regressão logística, como o de produzir estimativas pontuais e da variância dos estimadores muito distantes dos valores reais. Algumas técnicas para o seu diagnóstico foram apresentadas, sendo que com cada uma delas era possível identificar aspectos diferentes da multicolinearidade. Descrevemos também os estimadores viesados, em cristas e em componentes principais, e, com o intuito de comparar a performance destes dois estimadores, foi feito um estudo de simulação e uma aplicação em dados reais. No estudo de simulação, concluiu-se que ambos os estimadores conseguiram reduzir de forma drástica o valor do erro quadrático médio e produzir menor variabilidade no seu erro padrão em relação ao observado pelo estimador de máxima verossimilhança. Quanto maior o nível de multicolinearidade e menor o tamanho da amostra, maior foi o ganho em utilizar os estimadores viesados. Na aplicação em dados reais, a conclusão foi que o estimador em cristas foi o que mais conseguiu reduzir o valor da estatística do teste, embora ambos os estimadores viesados tenham apresentado redução significativa.

Pudemos concluir que a vantagem de se utilizar esses estimadores alternativos é que eles conseguem diminuir os efeitos da multicolinearidade e melhorar a precisão e acurácia a partir das estimativas dos efeitos das covariáveis. Além disso, ambos estimadores são de fácil obtenção dos programas de regressão logística, já que eles são simples transformações do estimador de máxima verossimilhança.

Como sugestões para trabalhos futuros recomandamos o estudo e a comparação de outros estimadores viesados, como o de Stein, primeiramente proposto em Stein (1960) e utilizado em Schaefer (1986) como uma extensão do estimador original em modelos de regressão logística. Este estimador encolhe o estimador de máxima verossimilhança e reduz significati- 
vamente a variância estimada dos parâmetros do modelo. Esse estimador não é amplamente divulgado e, por isso, se deu preferência aos outros dois estimadores apresentados nessa dissertação. Além disso, sugere-se a extensão de todo esse trabalho para a classe dos Modelos Lineares Generalizados. Em Mackinnon e Puterman (1989), por exemplo, é investigada a multicolinearidade e em Segerstedt (1992) e Marx e Smith (1990) são apresentados, respectivamente, os estimadores em cristas e em componentes principais nesta classe de modelos. 


\section{Apêndice A}

\section{Stepwise na Regressão Logística}

O método de stepwise é amplamente utilizado na construção de modelos de regressão logística com o intuito de diminuir o número de covariáveis. A seguir será ilustrado passo a passo o algoritmo de seleção de variáveis forward seguido do algoritmo de exclusão backward, de acordo com Hosmer e Lemeshow (2000).

- Passo (0): Neste passo é ajustado um modelo somente com o intercepto, dado por:

$$
\pi\left(\mathbf{x}_{i}\right)=\frac{\exp \left(\beta_{0}\right)}{1+\exp \left(\beta_{0}\right)}, i=1,2, \ldots, n
$$

e o log da sua verossimilhança, $L_{0}$, é calculado. Em seguida, um novo modelo com o intercepto e com cada uma das $p$-1 possíveis covariáveis é ajustado a fim de se comparar as suas respectivas log-verossimilhanças. Dessa forma, o modelo será dado por:

$$
\pi\left(\mathbf{x}_{i}\right)=\frac{\exp \left(\beta_{0}+\beta_{j} \mathbf{x}_{i j}\right)}{1+\exp \left(\beta_{0}+\beta_{j} \mathbf{x}_{i j}\right)}, i=1,2, \ldots, n, j=1, \ldots, p-1
$$

Seja $L_{j}^{(0)}, j=1, \ldots, p-1$, o $\log$ da verossimilhança do modelo ajustado com o intercepto e $\mathbf{x}_{j}$ no passo zero e seja:

$$
G_{j}^{(0)}=-2\left(L_{0}-L_{j}^{(0)}\right)
$$

o valor da estatística do teste de razão de verossimilhanças do modelo contendo o intercepto e $\mathbf{x}_{j}$ versus o modelo contendo somente o intercepto, com $p_{j}^{(0)}$ sendo o pvalor deste teste, determinado por $P\left[\chi_{(\nu)}^{2}>G_{j}^{(0)}\right]=p_{j}^{(0)}, \nu=1$ se $\mathbf{x}_{j}$ for contínua e $\nu=m-1$ se $\mathbf{x}_{j}$ for categórica com $m$ categorias. A variável mais importante, $\mathbf{x}_{e_{1}}$, será aquela com menor p-valor, ou seja, teremos:

$$
p_{e_{1}}^{(0)}=\min \left(p_{1}^{(0)}, \ldots, p_{p-1}^{(0)}\right)
$$

Porém, como a variável ter o menor p-valor não significa ela ser estatisticamente sig- 
nificante, será preciso comparar $p_{e_{1}}^{(0)}$ com o nível de significância pré-definido, $p_{e}$. Se $p_{e_{1}}^{(0)}<p_{e}$, a variável será considerada como significante e o algoritmo avança para o passo (1); caso contrário, o algoritmo para e o modelo final será composto somente pelo intercepto.

- Passo (1): Este passo começa com o modelo ajustado contendo o intercepto e a variá$\overline{\text { vel } \mathbf{x}_{e_{1}} \text {. Seja }} L_{e_{1}}^{(1)}$ o log da verossimilhança deste modelo. Para determinar qual das $p$-2 variáveis restantes são importantes dado que a variável $\mathbf{x}_{e_{1}}$ já está presente no modelo, será necessário ajustar $p$-2 novos modelos contendo o intercepto, $\mathbf{x}_{e_{1}}$ e $\mathbf{x}_{j}, j=1, \ldots, p-1$ e $j \neq e_{1}$, ou seja, serão ajustados os modelos:

$$
\pi\left(\mathbf{x}_{i}\right)=\frac{\exp \left(\beta_{0}+\beta_{e_{1}} \mathbf{x}_{e_{1}}+\beta_{j} \mathbf{x}_{j}\right)}{1+\exp \left(\beta_{0}+\beta_{e_{1}} \mathbf{x}_{i e_{1}}+\beta_{j} \mathbf{x}_{i j}\right)}, i=1,2, \ldots, n, j=1, \ldots, p-1, j \neq e_{1}
$$

Seja $L_{e_{1}, j}^{(1)}$ o log da verossimilhança desses modelos e:

$$
G_{j}^{(1)}=-2\left(L_{e_{1}}^{(1)}-L_{e_{1}, j}^{(1)}\right)
$$

o valor da estatística dos testes de razão de verossimilhanças do modelo contendo o intercepto, $\mathbf{x}_{e_{1}}$ e $\mathbf{x}_{j}$ versus o modelo contendo somente o intercepto e $\mathbf{x}_{e_{1}}$, com $p_{j}^{(1)}$ sendo o p-valor destes testes. Se a variável com o menor p-valor neste passo for denominada $\mathbf{x}_{\mathbf{e}_{2}}$ e se $p_{e_{2}}^{(1)}<p_{e}$ então o algoritmo segue para o passo (2); caso contrário o algoritmo para.

- Passo (2): Este passo começa com o modelo ajustado contendo o intercepto e as variáveis $\mathbf{x}_{e_{1}}$ e $\mathbf{x}_{e_{2}}$. É possível que uma vez que a variável $\mathbf{x}_{e_{2}}$ foi adicionada no modelo, a variável $\mathbf{x}_{e_{1}}$ seja não mais significante. Neste passo, portanto, é necessário incluir o algoritmo de eliminação backward. Este algoritmo ajusta um modelo sem as variáveis adicionadas nos passos anteriores e calcula a importância de cada uma. Seja $L_{-e_{j}}^{(2)}$ o $\log$ da verossimilhança do modelo sem a variável $\mathbf{x}_{e_{j}}$ e:

$$
G_{-j}^{(2)}=-2\left(L_{-e_{j}}^{(2)}-L_{e_{1}, e_{2}}^{(2)}\right)
$$

o valor da estatística dos testes de razão de verossimilhanças assim como definido previamente, com os respectivos $\mathrm{p}$-valores dados por $p_{-e_{j}}^{(2)}$. Para verificar se a variável deve ou não sair, o algoritmo seleciona aquela com o maior p-valor. Denotando-a por $\mathbf{x}_{r_{2}}$ e sendo:

$$
p_{r_{2}}^{(2)}=\max \left(p_{-e_{j}}^{(2)}, p_{-e_{2}}^{(2)}\right),
$$

para excluir essa variável do modelo o algoritmo compara $p_{r_{2}}^{(2)}$ com um segundo nível de significância pré-definido, $p_{R}$, que indica o mínimo nível de contribuição das variáveis 
para que elas possam continuar no modelo. Se $p_{r_{2}}^{(2)}>p_{R}$, então $\mathbf{x}_{r_{2}}$ será removida do modelo; caso contrário, $\mathbf{x}_{r_{2}}$ continuará no modelo. Em ambos os casos o algoritmo segue para a etapa de seleção de variáveis. Na fase forward cada uma das $p$-3 variáveis restantes são utilizadas para ajustar novos modelos contendo o intercepto e as variáveis $\mathbf{x}_{e_{1}}, \mathbf{x}_{e_{2}}$ e $\mathbf{x}_{j}, j=1, \ldots, p$-1 e $j \neq e_{1}, e_{2}$, ou seja, o modelo será dado por:

$$
\pi\left(\mathbf{x}_{i}\right)=\frac{\exp \left(\beta_{0}+\beta_{e_{1}} \mathbf{x}_{e_{1}}+\beta_{e_{2}} \mathbf{x}_{e_{2}}+\beta_{j} \mathbf{x}_{j}\right)}{1+\exp \left(\beta_{0}+\beta_{e_{1}} \mathbf{x}_{e_{1}}+\beta_{e_{2}} \mathbf{x}_{e_{2}}+\beta_{j} \mathbf{x}_{j}\right)}, i=1,2, \ldots, n, j=1, \ldots, p-1, j \neq e_{1}, e_{2}
$$

O algoritmo calculará o log da verossimilhança de cada modelo, o valor das estatísticas dos testes da razão de verossilhança e os correspondentes p-valores. Se a variável com o menor p-valor neste passo for denominada $\mathbf{x}_{e_{3}}$ e se $p_{e_{3}}^{(1)}<p_{e}$ então o algoritmo segue para o passo (3); caso contrário o algoritmo para.

- Passo (3): Este passo é idêntico ao passo (2) e o processo continuará desta forma até o ĺtimo passo, denominado (S).

- Passo (S): Este passo ocorrerá quando: (1) todas as p-1 variáveis entrarem no modelo ou (2) todas as variáveis do modelo tem p-valor de eliminação menor do que $p_{R}$ e todas as variáveis que não entraram no modelo tem p-valor de entrada maior do que $p_{E}$. 
78 APÊNDICE A 


\section{Apêndice B}

\section{Código em R do Estudo de Simulação}

-Bibliotecas necessárias-

library(MASS)

library(stats)

library(SMIR)

-Parâmetros iniciais-

replicas $<-1000$

tamanho_amostra $<-\mathrm{c}(100,500,1000)$

qtde_covars $<-\mathrm{c}(5,10,15)$

multicol <- matrix $(\mathrm{c}(0,0.45,0.142,-1,-1,0,0.45,0.45,0.142,-1,0,0.45,0.142,0.142,-1$, $0,0.45,0.142,0.142,0.142,0,0.45,0.45,0.142,0.142,0,0.45,0.45,0.45,0.142), 5,6)$

-Matriz em que serão guardadas medidas resumo dos EQMs.

tabela_resumo1 $<-\operatorname{matrix}(0,36,6)$

colnames(tabela_resumo1) <- list("Media_EQM_EMV","DesvPad_EQM_EMV", "Media_EQM_Ridge","DesvPad_EQM_Ridge", "Media_EQM_CP", "DesvPad_EQM_CP")

-Matriz em que serão guardadas a quantidade de vezes que cada EQMs foi menor.

tabela_resumo $2<-$ matrix $(0,36,3)$

colnames(tabela_resumo2) <- list("EQM_EMV_Menor", "EQM_Ridge_Menor",

"EQM_CP_Menor")

-Matriz em que serão guardadas medidas descritivas da multicolidade.

medidas_multic $<-\operatorname{matrix}(0,36,4)$

colnames(medidas_multic) <- list("Media_R2", "DesvPad_R2", "Media_IndCondicao",

"DesvPad_IndCondicao")

-Matriz em que serão guardadas a quantidade de parâmetros com sinal positivo.

tabela_resumo3 <- $\operatorname{matrix}(0,36,3)$ 
colnames(tabela_resumo3) <- list("Sinal_EMV", "Sinal_Ridge", "Sinal_CP")

linha_tab_resumo $<-1$

for (i in 1:length(tamanho_amostra)) \{

for $(\mathrm{j}$ in $1:$ length(qtde_covars $))\{$

for (k in 1:nrow(multicol) $)\{$

if $((\mathrm{j}==1 \& \mathrm{k}<=3 \&$ multicol[ $[\mathrm{k}, 1]>=0) \mid(\mathrm{j}==2 \& \mathrm{k}<=4 \&$ multicol[ $[\mathrm{k}, 2]>=0) \mid(\mathrm{j}==3 \&$

$\mathrm{k}<=5 \&$ multicol $[\mathrm{k}, 4]>=0))\{$

-Vetor em que serão guardados os índices de condição.

indice_condicao $<-\operatorname{rep}(0$,replicas $)$

-Vetor em que serão guardados os máximos de R2.

r2_max $<-\operatorname{rep}(0$,replicas $)$

-Vetor em que serão guardados os EQMs.

eqm $<-$ matrix $(0$,replicas, 3$)$

colnames(eqm) <- list("EQM_EMV","EQM_Ridge",EQM_CP')

-Matriz em que serão guardados as frequências de escolha de cada lambda.

lambda_ridge $<-$ matrix (0,replicas, 6 )

coeficientes_emv $<-\operatorname{matrix}(0$,replicas,qtde_covars $[\mathrm{j}]+1)$

coeficientes_ridge $<-$ matrix $(0$,replicas,qtde_covars $[\mathrm{j}]+1)$

coeficientes_cp $<-\operatorname{matrix}(0$, replicas, qtde_covars[j] +1$)$

cont_sinal_emv $<-0$

cont_sinal_ridge $<-0$

cont_sinal_cp $<-0$

for ( $\mathrm{r}$ in 1:replicas) \{

-Valor original dos betas-

if $(\mathrm{j}==1)$ beta $<-\operatorname{cbind}(1,1,1,1,1,1)$

if $(\mathrm{j}==2)$ beta $<-\operatorname{cbind}(1,1,1,1,1,1,1,1,1,1,1)$

if $(\mathrm{j}==3)$ beta $<-\operatorname{cbind}(1,1,1,1,1,1,1,1,1,1,1,1,1,1,1,1)$

-Gera as covariáveis-

$\operatorname{aux}=0$

-Essa condição existe caso o modelo não convirja. Se isso ocorrer, gera uma nova base.

while $($ aux $==0)\{$

if $(\mathrm{j}==1 \&$ multicol[ $[\mathrm{k}, 1]>=0)\{$

$\mathrm{x} 1<$ - runif(tamanho_amostra[i], $\min =0, \max =1$ )

$\mathrm{x} 2<-$ runif(tamanho_amostra[i], $\min =0, \max =1)$

$\mathrm{x} 3<$ - runif(tamanho_amostra[i], $\min =0, \max =1$ ) 
$\mathrm{x} 4<$-runif(tamanho_amostra[i], $\min =0, \max =1)$

if $($ multicol $[\mathrm{k}, 1]>0) \times 5<-(\mathrm{x} 3+\mathrm{x} 4+($ multicol$[\mathrm{k}, 1] *$ runif(tamanho_amostra[i], min=0, $\max =1))$ ) -introduz a multicolinearidade.

else $\mathrm{x} 5<$-runif(tamanho_amostra[i], $\min =0, \max =1)$-sem multicolinearidade.

$\mathrm{x}<-$ cbind $(\mathrm{x} 1, \mathrm{x} 2, \mathrm{x} 3, \mathrm{x} 4, \mathrm{x} 5)$

\}

if $(\mathrm{j}==2 \&$ multicol $[\mathrm{k}, 2]>=0)\{$

$\mathrm{x} 1<$-runif $($ tamanho_amostra[i], $\min =0, \max =1)$

$\mathrm{x} 2<$-runif(tamanho_amostra[i], $\min =0, \max =1)$

$\mathrm{x} 3<-$ runif(tamanho_amostra[i], $\min =0, \max =1$ )

$\mathrm{x} 4<-$ runif(tamanho_amostra[i], $\min =0, \max =1)$

if $($ multicol $[\mathrm{k}, 2]>0) \times 5<-(\mathrm{x} 3+\mathrm{x} 4+($ multicol$[\mathrm{k}, 2] *$ runif(tamanho_amostra[i], min $=0$, $\max =1))$ ) -introduz a multicolinearidade.

else $\mathrm{x} 5<-$ runif(tamanho_amostra[i], $\min =0, \max =1)$-sem multicolinearidade.

$\mathrm{x} 6<$-runif(tamanho_amostra[i], $\min =0, \max =1$ )

$\mathrm{x} 7<$-runif(tamanho_amostra[i], $\min =0, \max =1$ )

$\mathrm{x} 8<$-runif(tamanho_amostra[i], $\min =0, \max =1$ )

$\mathrm{x} 9<-\operatorname{runif}($ tamanho_amostra[i], $\min =0, \max =1)$

if $($ multicol $[\mathrm{k}, 3]>0) \mathrm{x} 10<-(\mathrm{x} 8+\mathrm{x} 9+($ multicol $[\mathrm{k}, 3] *$ runif(tamanho_amostra[i], min $=0$, $\max =1)$ ) ) -introduz a multicolinearidade.

else $\mathrm{x} 10<$-runif(tamanho_amostra[i], $\min =0, \max =1)$-sem multicolinearidade.

$\mathrm{x}<-$ cbind $(\mathrm{x} 1, \mathrm{x} 2, \mathrm{x} 3, \mathrm{x} 4, \mathrm{x} 5, \mathrm{x} 6, \mathrm{x} 7, \mathrm{x} 8, \mathrm{x} 9, \mathrm{x} 10)$

\}

if $(\mathrm{j}==3 \&$ multicol $[\mathrm{k}, 4]>=0)\{$

$\mathrm{x} 1<-\operatorname{runif}($ tamanho_amostra[i] $, \min =0, \max =1)$

$\mathrm{x} 2<$-runif(tamanho_amostra[i], $\min =0, \max =1)$

$\mathrm{x} 3<-\operatorname{runif}($ tamanho_amostra[i], $\min =0, \max =1$ )

$\mathrm{x} 4<$ - runif(tamanho_amostra[i], $\min =0, \max =1$ )

if $($ multicol $[\mathrm{k}, 4]>0) \mathrm{x} 5<-(\mathrm{x} 3+\mathrm{x} 4+($ multicol$[\mathrm{k}, 4] *$ runif(tamanho_amostra[i], min $=0$, $\max =1))$ ) -introduz a multicolinearidade.

else $x 5<$-runif(tamanho_amostra[i], $\min =0, \max =1)$-sem multicolinearidade.

$\mathrm{x} 6<$-runif(tamanho_amostra[i], $\min =0, \max =1$ )

$x 7<$-runif(tamanho_amostra[i], $\min =0, \max =1)$

$\mathrm{x} 8<$-runif(tamanho_amostra[i], $\min =0, \max =1)$

$\mathrm{x} 9<$-runif(tamanho_amostra[i], $\min =0, \max =1)$

if $($ multicol $[\mathrm{k}, 5]>0) \mathrm{x} 10<-(\mathrm{x} 8+\mathrm{x} 9+(\operatorname{multicol}[\mathrm{k}, 5] *$ runif(tamanho_amostra[i], min $=0$, $\max =1))$ ) -introduz a multicolinearidade.

else $\mathrm{x} 10<$-runif(tamanho_amostra[i], min=0, max=1) -sem multicolinearidade.

$\mathrm{x} 11<-$ runif(tamanho_amostra[i], $\min =0, \max =1$ )

$\mathrm{x} 12<-\operatorname{runif}($ tamanho_amostra[i], $\min =0, \max =1)$ 
$\mathrm{x} 13<$-runif(tamanho_amostra[i], $\min =0, \max =1)$

$\mathrm{x} 14<-\operatorname{runif}($ tamanho_amostra[i], $\min =0, \max =1)$

if $($ multicol $[\mathrm{k}, 6]>0) \mathrm{x} 15<-(\mathrm{x} 13+\mathrm{x} 14+($ multicol[k,6]*runif(tamanho_amostra[i], min $=0$, $\max =1))$ ) -introduz a multicolinearidade.

else $\mathrm{x} 15<$ - runif(tamanho_amostra[i], min=0, $\max =1)$-sem multicolinearidade.

$\mathrm{x}<-$ cbind $(\mathrm{x} 1, \mathrm{x} 2, \mathrm{x} 3, \mathrm{x} 4, \mathrm{x} 5, \mathrm{x} 6, \mathrm{x} 7, \mathrm{x} 8, \mathrm{x} 9, \mathrm{x} 10, \mathrm{x} 11, \mathrm{x} 12, \mathrm{x} 13, \mathrm{x} 14, \mathrm{x} 15)$

\}

-Gera a variável resposta-

$\mathrm{x}<-10 * \mathrm{x}$

$\mathrm{x} \_$med $<-\operatorname{matrix}(0$, tamanho_amostra[i],qtde_covars $[\mathrm{j}])$

for ( $\mathrm{m}$ in 1:tamanho_amostra[i]) \{

$\mathrm{x} \_\operatorname{med}[\mathrm{m}, 1]<-(\mathrm{x}[\mathrm{m}, 1]-\operatorname{mean}(\mathrm{x}[, 1])) / \mathrm{sd}(\mathrm{x}[, 1])$

$\mathrm{x} \_$med $[\mathrm{m}, 2]<-(\mathrm{x}[\mathrm{m}, 2]-\operatorname{mean}(\mathrm{x}[, 2])) / \mathrm{sd}(\mathrm{x}[, 2])$

$\mathrm{x} \_\operatorname{med}[\mathrm{m}, 3]<-(\mathrm{x}[\mathrm{m}, 3]-\operatorname{mean}(\mathrm{x}[, 3])) / \mathrm{sd}(\mathrm{x}[, 3])$

$\mathrm{x} \_\operatorname{med}[\mathrm{m}, 4]<-(\mathrm{x}[\mathrm{m}, 4]-\operatorname{mean}(\mathrm{x}[, 4])) / \mathrm{sd}(\mathrm{x}[, 4])$

x_med $[\mathrm{m}, 5]<-(\mathrm{x}[\mathrm{m}, 5]-\operatorname{mean}(\mathrm{x}[, 5])) / \mathrm{sd}(\mathrm{x}[, 5])$

if $(\mathrm{j}>1)\{$

x_med $[\mathrm{m}, 6]<-(\mathrm{x}[\mathrm{m}, 6]-\operatorname{mean}(\mathrm{x}[, 6])) / \mathrm{sd}(\mathrm{x}[, 6])$

$x_{-} \operatorname{med}[\mathrm{m}, 7]<-(\mathrm{x}[\mathrm{m}, 7]-\operatorname{mean}(\mathrm{x}[, 7])) / \operatorname{sd}(\mathrm{x}[, 7])$

$\mathrm{x} \_$med $[\mathrm{m}, 8]<-(\mathrm{x}[\mathrm{m}, 8]-\operatorname{mean}(\mathrm{x}[, 8])) / \operatorname{sd}(\mathrm{x}[, 8])$

x_med $[\mathrm{m}, 9]<-(\mathrm{x}[\mathrm{m}, 9]-\operatorname{mean}(\mathrm{x}[, 9])) / \mathrm{sd}(\mathrm{x}[, 9])$

$\mathrm{x} \_\operatorname{med}[\mathrm{m}, 10]<-(\mathrm{x}[\mathrm{m}, 10]-\operatorname{mean}(\mathrm{x}[, 10])) / \mathrm{sd}(\mathrm{x}[, 10])$

\}

if $(\mathrm{j}==3)\{$

$\mathrm{x} \_\operatorname{med}[\mathrm{m}, 11]<-(\mathrm{x}[\mathrm{m}, 11]-\operatorname{mean}(\mathrm{x}[, 11])) / \operatorname{sd}(\mathrm{x}[, 11])$

$\mathrm{x} \_\operatorname{med}[\mathrm{m}, 12]<-(\mathrm{x}[\mathrm{m}, 12]-\operatorname{mean}(\mathrm{x}[, 12])) / \operatorname{sd}(\mathrm{x}[, 12])$

x_med $[\mathrm{m}, 13]<-(\mathrm{x}[\mathrm{m}, 13]-\operatorname{mean}(\mathrm{x}[, 13])) / \operatorname{sd}(\mathrm{x}[, 13])$

$\mathrm{x} \_$med $[\mathrm{m}, 14]<-(\mathrm{x}[\mathrm{m}, 14]-\operatorname{mean}(\mathrm{x}[, 14])) / \operatorname{sd}(\mathrm{x}[, 14])$

$\mathrm{x} \_\operatorname{med}[\mathrm{m}, 15]<-(\mathrm{x}[\mathrm{m}, 15]-\operatorname{mean}(\mathrm{x}[, 15])) / \operatorname{sd}(\mathrm{x}[, 15])$

\}

\}

y_aux $<$-matrix $(0$, tamanho_amostra $[\mathrm{i}], 2)$

for ( $m$ in 1:tamanho_amostra[i] $)\{$-calcula o valor de pi.

if $(\mathrm{j}==1) \mathrm{y} \_$aux $[\mathrm{m}, 1]<-\exp \left(1+\mathrm{x} \_\right.$med $[\mathrm{m}, 1]+\mathrm{x} \_$med $[\mathrm{m}, 2]+\mathrm{x} \_$med $[\mathrm{m}, 3]+\mathrm{x} \_\mathrm{med}[\mathrm{m}, 4]$

$\left.+\mathrm{x} \_\operatorname{med}[\mathrm{m}, 5]\right) /\left(1+\exp \left(1+\mathrm{x} \_\right.\right.$med $[\mathrm{m}, 1]+\mathrm{x} \_$med $[\mathrm{m}, 2]+\mathrm{x} \_$med $[\mathrm{m}, 3]+\mathrm{x} \_$med $[\mathrm{m}, 4]$

$\left.\left.+\mathrm{x}_{-} \operatorname{med}[\mathrm{m}, 5]\right)\right)$

if $(\mathrm{j}==2) \mathrm{y} \_$aux $[\mathrm{m}, 1]<-\exp \left(1+\mathrm{x} \_\right.$med $[\mathrm{m}, 1]+\mathrm{x} \_$med $[\mathrm{m}, 2]+\mathrm{x} \_$med $[\mathrm{m}, 3]+\mathrm{x} \_\mathrm{med}[\mathrm{m}, 4]$

$+\mathrm{x} \_$med $[\mathrm{m}, 5]+\mathrm{x} \_$med $[\mathrm{m}, 6]+\mathrm{x} \_$med $[\mathrm{m}, 7]+\mathrm{x} \_$med $[\mathrm{m}, 8]+\mathrm{x} \_$med $[\mathrm{m}, 9]+\mathrm{x} \_$med $\left.[\mathrm{m}, 10]\right)$ 
$/\left(1+\exp \left(1+\mathrm{x} \_\right.\right.$med $[\mathrm{m}, 1]+\mathrm{x} \_$med $[\mathrm{m}, 2]+\mathrm{x} \_\operatorname{med}[\mathrm{m}, 3]+\mathrm{x} \_\operatorname{med}[\mathrm{m}, 4]+\mathrm{x} \_$med $[\mathrm{m}, 5]+$ $\mathrm{x} \_$med $[\mathrm{m}, 6]+\mathrm{x} \_$med $[\mathrm{m}, 7]+\mathrm{x} \_$med $[\mathrm{m}, 8]+\mathrm{x} \_$med $\left.\left.[\mathrm{m}, 9]+\mathrm{x} \_\operatorname{med}[\mathrm{m}, 10]\right)\right)$

if $(\mathrm{j}==3) \mathrm{y} \_$aux $[\mathrm{m}, 1]<-\exp \left(1+\mathrm{x} \_\right.$med $[\mathrm{m}, 1]+\mathrm{x} \_\operatorname{med}[\mathrm{m}, 2]+\mathrm{x} \_\operatorname{med}[\mathrm{m}, 3]+\mathrm{x} \_\operatorname{med}[\mathrm{m}, 4]$ $+\mathrm{x} \_$med $[\mathrm{m}, 5]+\mathrm{x} \_\operatorname{med}[\mathrm{m}, 6]+\mathrm{x} \_\operatorname{med}[\mathrm{m}, 7]+\mathrm{x} \_$med $[\mathrm{m}, 8]+\mathrm{x} \_\operatorname{med}[\mathrm{m}, 9]+\mathrm{x} \_$med $[\mathrm{m}, 10]$ $+\mathrm{x} \_$med $[\mathrm{m}, 11]+\mathrm{x} \_\operatorname{med}[\mathrm{m}, 12]+\mathrm{x} \_\operatorname{med}[\mathrm{m}, 13]+\mathrm{x} \_\operatorname{med}[\mathrm{m}, 14]+\mathrm{x} \_$med $\left.[\mathrm{m}, 15]\right) /($ $1+\exp \left(1+\mathrm{x} \_\right.$med $[\mathrm{m}, 1]+\mathrm{x} \_$med $[\mathrm{m}, 2]+\mathrm{x} \_$med $[\mathrm{m}, 3]+\mathrm{x} \_\operatorname{med}[\mathrm{m}, 4]+\mathrm{x} \_\operatorname{med}[\mathrm{m}, 5]+$ $\mathrm{x} \_$med $[\mathrm{m}, 6]+\mathrm{x} \_$med $[\mathrm{m}, 7]+\mathrm{x} \_$med $[\mathrm{m}, 8]+\mathrm{x} \_$med $[\mathrm{m}, 9]+\mathrm{x} \_$med $[\mathrm{m}, 10]+\mathrm{x} \_$med $[\mathrm{m}, 11]$ $+\mathrm{x} \_$med $[\mathrm{m}, 12]+\mathrm{x} \_$med $[\mathrm{m}, 13]+\mathrm{x} \_$med $[\mathrm{m}, 14]+\mathrm{x} \_$med $\left.\left.[\mathrm{m}, 15]\right)\right)$

\}

-A variável resposta é Binomial com parâmetro pi.

for ( $\mathrm{m}$ in 1:tamanho_amostra[i] $)\{$

y_aux $[\mathrm{m}, 2]<-\operatorname{rbinom}\left(1,1, \mathrm{y}_{\text {_aux }}[\mathrm{m}, 1]\right)$

\}

$\mathrm{y}<-$ as.factor $\left(\mathrm{y} \_\right.$aux $\left.[, 2]\right)$

-Modelo-

if $(\mathrm{j}==1)$ modelo $<-\operatorname{glm}\left(\mathrm{y} \sim \mathrm{x} \_\right.$med[,1] $+\mathrm{x} \_$med[,2] $+\mathrm{x} \_$med[,3] $+\mathrm{x} \_$med[,4] + $\mathrm{x} \_$med[,5], family=binomial, $\left.\mathrm{x}=\mathrm{TRUE}\right)$

if $(\mathrm{j}==2)$ modelo $<-\operatorname{glm}\left(\mathrm{y} \sim \mathrm{x} \_\right.$med[,1] $+\mathrm{x} \_$med[,2] $+\mathrm{x} \_$med[,3] $+\mathrm{x} \_$med[,4] + $\mathrm{x} \_$med $[, 5]+\mathrm{x} \_$med[,6] $+\mathrm{x} \_$med $[, 7]+\mathrm{x} \_$med[,8] $+\mathrm{x} \_$med[,9] $+\mathrm{x} \_$med[, 10], family=binomial, $\mathrm{x}=\mathrm{TRUE})$

if $(\mathrm{j}==3)$ modelo $<-\operatorname{glm}\left(\mathrm{y} \sim \mathrm{x} \_\right.$med[,1] $+\mathrm{x} \_$med[,2] $+\mathrm{x} \_$med[,3] $+\mathrm{x} \_$med[,4] + $\mathrm{x} \_$med[,5] $+\mathrm{x} \_$med[,6] $+\mathrm{x} \_$med[,7] $+\mathrm{x} \_$med[,8] $+\mathrm{x} \_$med[,9] $+\mathrm{x} \_$med[,10] $+\mathrm{x} \_$med[,11] $+\mathrm{x} \_$med[,12] $+\mathrm{x} \_$med[,13] $+\mathrm{x} \_$med[,14] $+\mathrm{x} \_$med[,15], family=binomial, $\mathrm{x}=$ TRUE)

-Erro Quadrático do Modelo de Regressão (estimador de máxima verossimilhança).

eqm $[\mathrm{r}, 1]<-($ beta - coefficients(modelo) $) \% * \%(\mathrm{t}($ beta - coefficients (modelo) $))$

coeficientes_emv[r,] <- coefficients(modelo)

-Verifica se o modelo convergiu.

if $($ modelo $\$$ converged $==1 \&$ eqm $[\mathrm{r}, 1]<1000000)\{$

aux $<-1$

write.table(cbind(y_aux[,2],x_med), file=paste("bases/", "Cenario_", i, j, k, “/Base_", r,".txt", $\left.\left.\operatorname{sep}={ }^{69}\right)\right)$

\}

\}-fecha o while

-Identificação da Multicolinearidade-

-Decomposição em Valores Singulares.

-Matriz diagonal da raiz quadrada dos pesos. 
sqrt_w $<-$ diag(sqrt(modelo\$weights))

-Matriz K

matriz_k <-sqrt_w\%*\%modelo $\$ \mathrm{x}$

-SDV da matriz $\mathrm{k}$

svd_k $<-\operatorname{svd}($ matriz_k)

-Índice de Condição.

indice_condicao $[\mathrm{r}]<-\max \left(\mathrm{svd} \_\mathrm{k} \$ \mathrm{~d}\right) / \min \left(\mathrm{svd} \_\mathrm{k} \$ \mathrm{~d}\right)$

-R2 máximo.

if $(\mathrm{j}==1)\{$

$\mathrm{r} 2<-\operatorname{rep}(0,5)$

r2[1] <- R2(lm(x_med[,1] x_med[,2] + x_med[,3] + x_med[,4] + x_med[,5])

$\mathrm{r} 2[2]<-\mathrm{R} 2\left(\operatorname{lm}\left(\mathrm{x} \_\right.\right.$med[,2] $\sim \mathrm{x} \_$med[,1] $+\mathrm{x} \_$med[,3] $+\mathrm{x} \_$med[,4] $+\mathrm{x} \_$med[,5] $\left.)\right)$

r2[3] <- R2(lm(x_med[,3] x_med[,1] + x_med[,2] + x_med[,4] + x_med[,5])

$\mathrm{r} 2[4]<-\mathrm{R} 2\left(\operatorname{lm}\left(\mathrm{x} \_\right.\right.$med[$[, 4] \sim \mathrm{x} \_$med[,1] $+\mathrm{x} \_$med $[, 2]+\mathrm{x} \_$med $[, 3]+\mathrm{x} \_$med $\left.\left.[, 5]\right)\right)$

$\mathrm{r} 2[5]<-\mathrm{R} 2\left(\mathrm{~lm}\left(\mathrm{x} \_\right.\right.$med $[, 5] \sim \mathrm{x} \_$med $[, 1]+\mathrm{x} \_$med $[, 2]+\mathrm{x} \_$med[,3] $+\mathrm{x} \_$med[,4] $\left.)\right)$

$\mathrm{r} 2 \_\max [\mathrm{r}]<-\max (\mathrm{r} 2)$

\}

if $(\mathrm{j}==2)\{$

$\mathrm{r} 2<-\operatorname{rep}(0,10)$

r2[1] <- R2 $\left(\operatorname{lm}\left(\mathrm{x} \_\right.\right.$med[,1] x_med[,2] + x_med[,3] + x_med[,4] + x_med[,5] + x_med[,6]

$+\mathrm{x} \_$med[,7] $+\mathrm{x} \_$med[,8] $+\mathrm{x} \_$med[,9] $+\mathrm{x} \_$med[,10])

$\mathrm{r} 2[2]<-\mathrm{R} 2\left(\operatorname{lm}\left(\mathrm{x} \_\right.\right.$med[,2] $\mathrm{x} \_$med[, 1$]+\mathrm{x} \_$med[,3] $+\mathrm{x} \_$med[,4] $+\mathrm{x} \_$med[,5] $+\mathrm{x} \_$med[,6]

$+\mathrm{x} \_$med[,7] $+\mathrm{x} \_$med[,8] $+\mathrm{x} \_$med[,9] $+\mathrm{x} \_$med[,10])

$\mathrm{r} 2[3]<-\mathrm{R} 2\left(\mathrm{~lm}\left(\mathrm{x} \_\right.\right.$med[,3] $\sim \mathrm{x} \_$med[,1] $+\mathrm{x} \_$med[,2] $+\mathrm{x} \_$med $[, 4]+\mathrm{x} \_$med $[, 5]+\mathrm{x} \_$med[,6]

$+\mathrm{x} \_$med $[, 7]+\mathrm{x} \_$med $[, 8]+\mathrm{x} \_$med $[, 9]+\mathrm{x} \_$med $\left.\left.[, 10]\right)\right)$

$\mathrm{r} 2[4]<-\mathrm{R} 2\left(\operatorname{lm}\left(\mathrm{x} \_\right.\right.$med[, 4$] \sim \mathrm{x} \_$med[, 1$]+\mathrm{x} \_$med[,2] $+\mathrm{x} \_$med[,3] $+\mathrm{x} \_$med[,5] $+\mathrm{x} \_$med[,6]

$+\mathrm{x} \_$med[,7] $+\mathrm{x} \_$med[,8] $+\mathrm{x} \_$med[,9] $+\mathrm{x} \_$med[,10]))

$\mathrm{r} 2[5]<-\mathrm{R} 2\left(\operatorname{lm}\left(\mathrm{x} \_\right.\right.$med[,5] $\sim \mathrm{x} \_$med[,1] $+\mathrm{x} \_$med[,2] $+\mathrm{x} \_$med[,3] $+\mathrm{x} \_$med $[, 4]+\mathrm{x} \_$med[,6]

$+\mathrm{x} \_$med[,7] $+\mathrm{x} \_$med[,8] $+\mathrm{x} \_$med[,9] $+\mathrm{x} \_$med[,10])

$\mathrm{r} 2[6]<-\mathrm{R} 2\left(\operatorname{lm}\left(\mathrm{x} \_\right.\right.$med[,6] $\sim \mathrm{x} \_$med[, 1$]+\mathrm{x} \_$med[,2] $+\mathrm{x} \_$med[,3] $+\mathrm{x} \_$med[,4] $+\mathrm{x} \_$med[,5]

$+\mathrm{x} \_$med[,7] $+\mathrm{x} \_$med[,8] $+\mathrm{x} \_$med[,9] $+\mathrm{x} \_$med[,10]))

$\mathrm{r} 2[7]<-\mathrm{R} 2\left(\mathrm{~lm}\left(\mathrm{x} \_\right.\right.$med[,7] $\sim \mathrm{x} \_$med[,1] $+\mathrm{x} \_$med[,2] $+\mathrm{x} \_$med[,3] $+\mathrm{x} \_$med[,4] $+\mathrm{x} \_$med[,5]

$+\mathrm{x} \_$med[,6] $+\mathrm{x} \_$med[,8] $+\mathrm{x} \_$med[,9] $+\mathrm{x} \_$med[,10])

$\mathrm{r} 2[8]<-\mathrm{R} 2\left(\operatorname{lm}\left(\mathrm{x} \_\right.\right.$med[, 8$] \sim \mathrm{x} \_$med[, 1$]+\mathrm{x} \_$med[,2] $+\mathrm{x} \_$med[,3] $+\mathrm{x} \_$med $[, 4]+\mathrm{x} \_$med[,5]

$+\mathrm{x} \_$med[,6] $+\mathrm{x} \_$med[,7] $+\mathrm{x} \_$med[,9] $+\mathrm{x} \_$med[,10]))

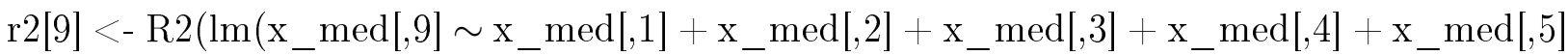

$+\mathrm{x} \_$med[,6] $+\mathrm{x} \_$med[,7] $+\mathrm{x} \_$med[,8] $+\mathrm{x} \_$med[,10])

$\mathrm{r} 2[10]<-\mathrm{R} 2\left(\mathrm{~lm}\left(\mathrm{x} \_\right.\right.$med $[, 10] \sim \mathrm{x} \_$med[,1] $+\mathrm{x} \_$med[,2] $+\mathrm{x} \_$med $[, 3]+\mathrm{x} \_$med $[, 4]+\mathrm{x} \_$med[,5]

$+\mathrm{x} \_$med $[, 6]+\mathrm{x} \_$med $[, 7]+\mathrm{x} \_$med $[, 8]+\mathrm{x} \_$med $\left.\left.[, 9]\right)\right)$

r2_max $[\mathrm{r}]<-\max (\mathrm{r} 2)$ 
\}

if $(\mathrm{j}==3)\{$

$\mathrm{r} 2<-\operatorname{rep}(0,15)$

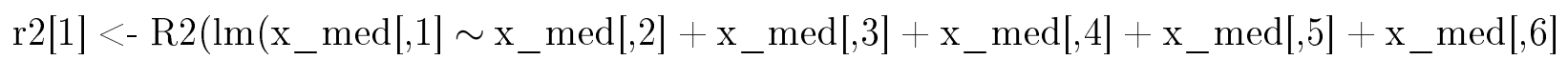
$+\mathrm{x} \_$med[,7] $+\mathrm{x} \_$med[,8] $+\mathrm{x} \_$med[,9] $+\mathrm{x} \_$med[,10] $+\mathrm{x} \_$med[,11] $+\mathrm{x} \_$med[,12] + $\mathrm{x} \_$med[,13] $+\mathrm{x} \_$med[,14] $+\mathrm{x} \_$med[,15]))

r2[2] <- R2 $(\mathrm{lm}$ (x_med[,2] x_med[,1] + x_med[,3] + x_med[,4] + x_med[,5] + x_med[,6] $+\mathrm{x} \_$med $[, 7]+\mathrm{x} \_$med[,8] $+\mathrm{x} \_$med[,9] $+\mathrm{x} \_$med $[, 10]+\mathrm{x} \_$med $[, 11]+\mathrm{x} \_$med $[, 12]+$ x_med[,13] + x_med[,14] + x_med[,15]))

r2[3] <- R2 $\left(\operatorname{lm}\left(\mathrm{x} \_\right.\right.$med[,3] x_med[,1] + x_med[,2] + x_med[,4] + x_med[,5] + x_med[,6] $+\mathrm{x} \_$med $[, 7]+\mathrm{x} \_$med[,8] $+\mathrm{x} \_$med[,9] $+\mathrm{x} \_$med[,10] $+\mathrm{x} \_$med[,11] $+\mathrm{x} \_$med $[, 12]+$ $\mathrm{x} \_$med[,13] $+\mathrm{x} \_$med[,14] $+\mathrm{x} \_$med[,15]))

r2[4] <- R2 (lm(x_med[,4] x_med[,1] + x_med[,2] + x_med[,3] + x_med[,5] + x_med[,6] $+\mathrm{x} \_$med[,7] $+\mathrm{x}$ _med[,8] $+\mathrm{x}$ _med[,9] $+\mathrm{x} \_$med[,10] $+\mathrm{x} \_$med[,11] $+\mathrm{x} \_$med $[, 12]+$ x_med[,13] + x_med[,14] + x_med[,15]))

r2[5] <- R2 (lm(x_med[,5] x_med[,1] + x_med[,2] + x_med[,3] + x_med[,4] + x_med[,6] $+\mathrm{x} \_$med[,7] $+\mathrm{x} \_$med[,8] $+\mathrm{x} \_$med[,9] $+\mathrm{x} \_$med[,10] $+\mathrm{x} \_$med[,11] $+\mathrm{x} \_$med[,12] + x_med[,13] + x_med[,14] + x_med[,15]))

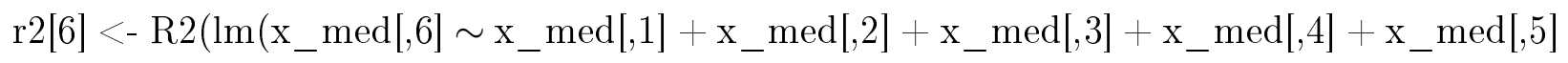
$+\mathrm{x} \_$med[,7] $+\mathrm{x}$ _med[,8] $+\mathrm{x}$ _med[,9] $+\mathrm{x} \_$med[,10] $+\mathrm{x} \_$med[,11] $+\mathrm{x} \_$med[,12] + x_med[,13] $+\mathrm{x} \_$med[,14] $+\mathrm{x} \_$med[,15]))

r2[7]<- R2 $\left(1 \mathrm{~lm}\left(\mathrm{x} \_\right.\right.$med $[, 7] \sim \mathrm{x} \_$med[,1] + x_med[,2] + x_med[,3] + x_med[,4] + x_med[,5] $+\mathrm{x} \_$med[,6] $+\mathrm{x} \_$med[,8] $+\mathrm{x} \_$med[,9] $+\mathrm{x} \_$med[,10] $+\mathrm{x} \_$med[,11] $+\mathrm{x} \_$med[,12] + x_med[,13] + x_med[,14] + x_med[,15]))

r2[8] <- R2 $\left(1 \mathrm{~lm}\left(\mathrm{x} \_\right.\right.$med $[, 8] \sim \mathrm{x} \_$med[,1] $+\mathrm{x} \_$med[,2] + x_med[,3] + x_med[,4] + x_med[,5] $+\mathrm{x} \_$med[,6] $+\mathrm{x} \_$med $[, 7]+\mathrm{x} \_$med[,9] $+\mathrm{x} \_$med $[, 10]+\mathrm{x} \_$med[,11] $+\mathrm{x} \_$med $[, 12]+$ x_med[,13] + x_med[,14] + x_med[,15]))

r2[9] <- R2(lm(x_med[,9] x_med[,1] + x_med[,2] + x_med[,3] + x_med[,4] + x_med[,5] $+\mathrm{x} \_$med[,6] $+\mathrm{x} \_$med[,7] $+\mathrm{x} \_$med[,8] $+\mathrm{x} \_$med[,10] $+\mathrm{x} \_$med $[, 11]+\mathrm{x} \_$med[,12] + x_med[,13] $+\mathrm{x} \_$med[,14] $+\mathrm{x} \_$med[,15]))

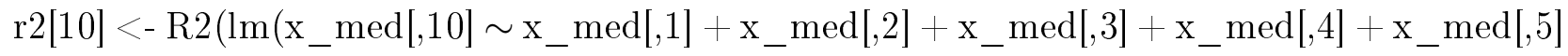

$+\mathrm{x} \_$med $[, 6]+\mathrm{x} \_$med[,7] $+\mathrm{x} \_$med[,8] $+\mathrm{x} \_$med $[, 9]+\mathrm{x} \_$med[,11] $+\mathrm{x} \_$med[,12] $+\mathrm{x} \_$med $[, 13]$

$+\mathrm{x} \_$med[,14] $+\mathrm{x} \_$med[,15]))

r2[11] <- R2 (lm(x_med[,11] x_med[,1] + x_med[,2] + x_med[,3] + x_med[,4] + x_med[,5]

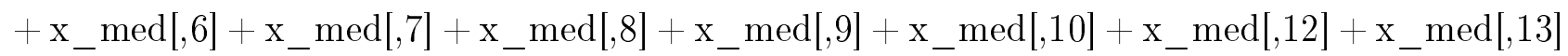

$+\mathrm{x} \_$med[,14] $+\mathrm{x} \_$med[,15]))

r2[12] <- R2 (lm(x_med[,12] x_med[,1] + x_med[,2] + x_med[,3] + x_med[,4] + x_med[,5]

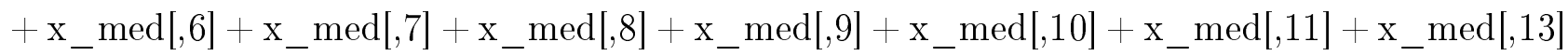

$+\mathrm{x} \_$med $[, 14]+\mathrm{x} \_$med[,15]))

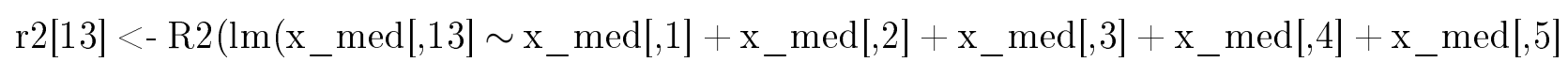


$+\mathrm{x} \_$med[,6] $+\mathrm{x} \_$med[,7] $+\mathrm{x} \_$med[,8] $+\mathrm{x} \_$med[,9] $+\mathrm{x} \_$med[,10] $+\mathrm{x} \_$med[,11] $+\mathrm{x} \_$med[,12]

$+\mathrm{x}$ _med[,14] $+\mathrm{x}$ _med[,15]))

$\mathrm{r} 2[14]<-\mathrm{R} 2\left(\mathrm{~lm}\left(\mathrm{x} \_\right.\right.$med[,14] $\sim \mathrm{x} \_$med $[, 1]+\mathrm{x} \_$med[,2] $+\mathrm{x} \_$med $[, 3]+\mathrm{x} \_$med[,4] $+\mathrm{x} \_$med $[, 5]$

$+\mathrm{x} \_$med[,6] $+\mathrm{x} \_$med[,7] $+\mathrm{x} \_$med[,8] $+\mathrm{x} \_$med[,9] $+\mathrm{x} \_$med[,10] $+\mathrm{x} \_$med[,11] $+\mathrm{x} \_$med[,12]

$+\mathrm{x} \_$med[,13] $+\mathrm{x} \_$med[,15]))

$\mathrm{r} 2[15]<-\mathrm{R} 2\left(\mathrm{~lm}\left(\mathrm{x} \_\right.\right.$med[,15] $\sim \mathrm{x} \_$med[,1] $+\mathrm{x} \_$med[,2] $+\mathrm{x} \_$med[,3] $+\mathrm{x} \_$med[,4] $+\mathrm{x} \_$med[,5]

$+\mathrm{x} \_$med[,6] $+\mathrm{x} \_$med[,7] $+\mathrm{x} \_$med[,8] $+\mathrm{x} \_$med $[, 9]+\mathrm{x} \_$med $[, 10]+\mathrm{x} \_$med $[, 11]+\mathrm{x} \_$med[,12]

$+\mathrm{x}_{-}$med[,13] $+\mathrm{x} \_$med[,14]))

r2_max $[\mathrm{r}]<-\max (\mathrm{r} 2)$

\}

-Regressão em Cristas-

-Calcula variáveis auxiliares.

resp <- as.numeric(as.vector $(\mathrm{y}))$

-Erro quadrático médio do desvio.

$\mathrm{s} 2<-(\mathrm{t}($ resp - modelo $\$$ fitted.values $) \% * \%($ resp - modelo $\$$ fitted.values $)) /($ nrow $($ modelo $\$ \mathrm{x})$

$\left.-\operatorname{ncol}\left(\mathrm{svd} \_\mathrm{k} \$ \mathrm{v}\right)-1\right)$

alpha_prod $<-1$

alpha $<-\operatorname{rep}\left(0\right.$, ncol $\left.\left(\operatorname{svd}_{-} \mathrm{k} \$ \mathrm{v}\right)\right)$

$\mathrm{m} 2<-\operatorname{rep}\left(0, \mathrm{ncol}\left(\mathrm{svd} \_\mathrm{k} \$ \mathrm{v}\right)\right)$

for $(\mathrm{m}$ in 1:ncol(svd_ $\mathrm{k} \$ \mathrm{v}))\{$

alpha_m <-svd_k $\$ \mathrm{v}[\mathrm{m}] \% * \$,$% modelo \$$ coefficients

alpha $[\mathrm{m}]<-$ alpha_m

alpha_prod $<-$ alpha_prod $*(($ alpha_m $) \wedge 2)$

$\mathrm{m} 2[\mathrm{~m}]<-\mathrm{s} 2 /(($ alpha_m $) \wedge 2)$

\}

vetor_s $<-\operatorname{rep}\left(0, \mathrm{ncol}\left(\mathrm{svd} \_\mathrm{k} \$ \mathrm{v}\right)\right)$

for $\left(\mathrm{m}\right.$ in $\left.1: \mathrm{ncol}\left(\mathrm{svd} \_\mathrm{k} \$ \mathrm{v}\right)\right)\{$

vetor_s $[\mathrm{m}]<-\left(\mathrm{svd}_{-} \mathrm{k} \$ \mathrm{~d}[\mathrm{~m}] * \mathrm{~s} 2\right) /\left(\left(\left(\operatorname{nrow}(\operatorname{modelo} \$ \mathrm{x})-\mathrm{ncol}\left(\mathrm{svd} \_\mathrm{k} \$ \mathrm{v}\right)-1\right) * \mathrm{~s} 2\right)+\left(\mathrm{svd} \_\mathrm{k} \$ \mathrm{~d}[\mathrm{~m}]\right.\right.$

$*(($ svd_k $\$ \mathrm{v}[\mathrm{m}] \% * \$,$% modelo \$$ coefficients $) \wedge 2)))$

\}

-Calcula os valores para lambda.

lambda_1<-s2/max(alpha)

lambda_2<-ncol(svd_k $\$ \mathrm{v}) /(\mathrm{t}($ modelo $\$$ coefficients $) \% * \%$ modelo $\$$ coefficients $)$

lambda_3<-s2/((alpha_prod $\left.) \wedge\left(1 / \operatorname{ncol}\left(\operatorname{svd} \_\mathrm{k} \$ \mathrm{v}\right)\right)\right)$

lambda_ $4<-\operatorname{median}(\mathrm{m} 2)$

lambda_5<- $\max ($ vetor_s)

-Modelos de Regressão em Cristas.

xwx $<-\mathrm{t}($ modelo $\$ \mathrm{x}) \% * \% \operatorname{diag}($ modelo $\$$ weights $) \% * \%$ modelo $\$ \mathrm{x}$ 


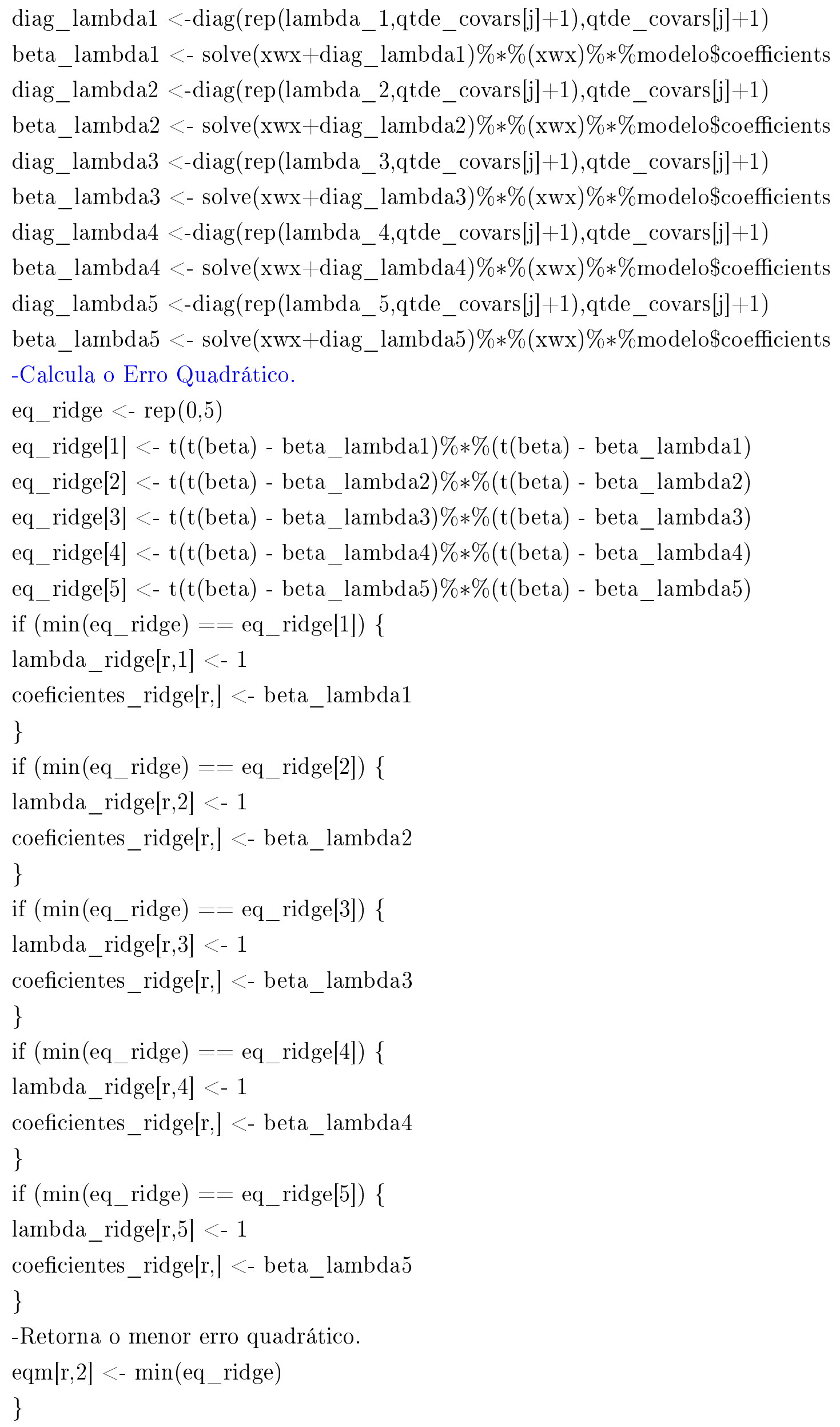


-Análise de Componentes Principais -

cont $<-0$

for $\left(\mathrm{m}\right.$ in 1:length $\left.\left(\mathrm{svd} \_\mathrm{k} \$ \mathrm{~d}\right)\right)\{$

if $\left(\left(\max \left(\mathrm{svd} \_\mathrm{k} \$ \mathrm{~d}\right) / \mathrm{svd} \_\mathrm{k} \$ \mathrm{~d}[\mathrm{~m}]\right)>10\right)$ cont $<-$ cont +1

\}

-Autovetores não associados às multicolinearidades.

autovetores_q $<-$ svd_k $\$ \mathrm{v}[, 1:($ length(svd_k $\$ \mathrm{~d})$-cont)]

-Autovalores não associados s̀ multicolinearidades.

autovalores_2 <- $\left(\mathrm{svd} \_\mathrm{k} \$ \mathrm{~d} \wedge(-2)\right)\left[1:\left(\right.\right.$ length $\left(\mathrm{svd} \_\mathrm{k} \$ \mathrm{~d}\right)$-cont $\left.)\right]$

-Matriz diagonal dos autovalores não associados s̀ multicolinearidades.

autovalores_q <-diag(autovalores_2,(length(svd_k $\$ d$ )-cont))

-X'WX^-1 considerando os autovalores não associados s̀ multicolinearidades.

xwx_q <- autovetores_q \%*\% autovalores_q \%*\% t(autovetores_q)

-Estimativas dos coeficientes de componentes principais.

beta_cp <- xwx_q \%*\% (t(matriz_k) $\% * \%$ matriz_k) $\% * \%$ modelo $\$$ coefficients

-Retorna o erro quadrático da Regressão em Componentes Principais.

eqm $[\mathrm{r}, 3]<-\mathrm{t}(\mathrm{t}($ beta $)$ - beta_cp)\%*\%(t(beta) - beta_cp)

coeficientes_cp $[\mathrm{r}]<$,- beta_cp

-Retorna a quantidade de vezes que o EQM de cada simulação foi menor.

if $($ eqm $[\mathrm{r}, 1]<=$ eqm $[\mathrm{r}, 2] \&$ eqm $[\mathrm{r}, 1]<=$ eqm[r,3]) tabela_resumo2[linha_tab_resumo,1] $<-$ tabela_resumo2[linha_tab_resumo,1] +1

if $($ eqm $[\mathrm{r}, 2]<$ eqm[r,1] \& eqm[r,2] $<$ eqm[r,3]) tabela_resumo2[linha_tab_resumo,2] <tabela_resumo2[linha_tab_resumo,2] +1

if $($ eqm $[r, 3]<$ eqm[r,2] \& eqm[r,3] $<$ eqm[r,1]) tabela_resumo2[linha_tab_resumo,3] <tabela_resumo2[linha_tab_resumo,3] +1

-Retorna a quantidade de coeficientes com sinal positivo.

if $(\mathrm{j}==1)$ qtde_coef $<-6$

if $(\mathrm{j}==2)$ qtde_coef $<-11$

if $(\mathrm{j}==3)$ qtde_coef $<-16$

for ( $\mathrm{p}$ in 1:qtde_coef) \{

if $($ coeficientes_emv $[\mathrm{r}, \mathrm{p}]>0)$ cont_sinal_emv $<-$ cont_sinal_emv +1

if $($ coeficientes_ridge $[\mathrm{r}, \mathrm{p}]>0)$ cont_sinal_ridge $<$ - cont_sinal_ridge +1

if $($ coeficientes_cp $[\mathrm{r}, \mathrm{p}]>0)$ cont_sinal_cp $<-$ cont_sinal_cp +1

\}

\}-fecha o laço das réplicas.

- Retorna as medidas resumo do EQM.

tabela_resumo1[linha_tab_resumo,1] $<-$ mean $($ eqm $[, 1])$

tabela_resumo1[linha_tab_resumo,2] $<-\operatorname{sd}($ eqm $[, 1])$ 
tabela_resumo1[linha_tab_resumo,3] $<-$ mean $($ eqm[,2])

tabela_resumo1[linha_tab_resumo,4] $<-\operatorname{sd}($ eqm[,2])

tabela_resumo1[linha_tab_resumo,5] $<-$ mean $($ eqm $[, 3])$

tabela_resumo1[linha_tab_resumo,6] $<-\operatorname{sd}($ eqm $[, 3])$

-Retorna as medidas resumo da multicolinearidade.

medidas_multic[linha_tab_resumo,1] $<-\operatorname{mean}\left(\mathrm{r} 2 \_\right.$max $)$

medidas_multic[linha_tab_resumo,2] $<-$ sd $\left(\mathrm{r} 2 \_\right.$max $)$

medidas_multic[linha_tab_resumo,3] $<-$ mean(indice_condicao)

medidas_multic[linha_tab_resumo,4] $<-$ sd(indice_condicao)

-Retorna a quantidade de coeficientes com o sinal positivo

tabela_resumo3[linha_tab_resumo,1] <- cont_sinal_emv

tabela_resumo3[linha_tab_resumo,2] <-cont_sinal_ridge)

tabela_resumo3[linha_tab_resumo,3] $<-$ cont_sinal_cp

linha_tab_resumo $<-$ linha_tab_resumo +1

\} -fecha a condição da multicolinearidade.

\}-fecha o laço da multicolinearidade.

\} -fecha o laço da qtde_covars.

\}-fecha o laço do tamanho_amostra. 
90 APÊNDICE B 


\section{Apêndice C}

\section{Código em R da Análise do Conjunto de Dados Reais}

-Leitura dos dados-

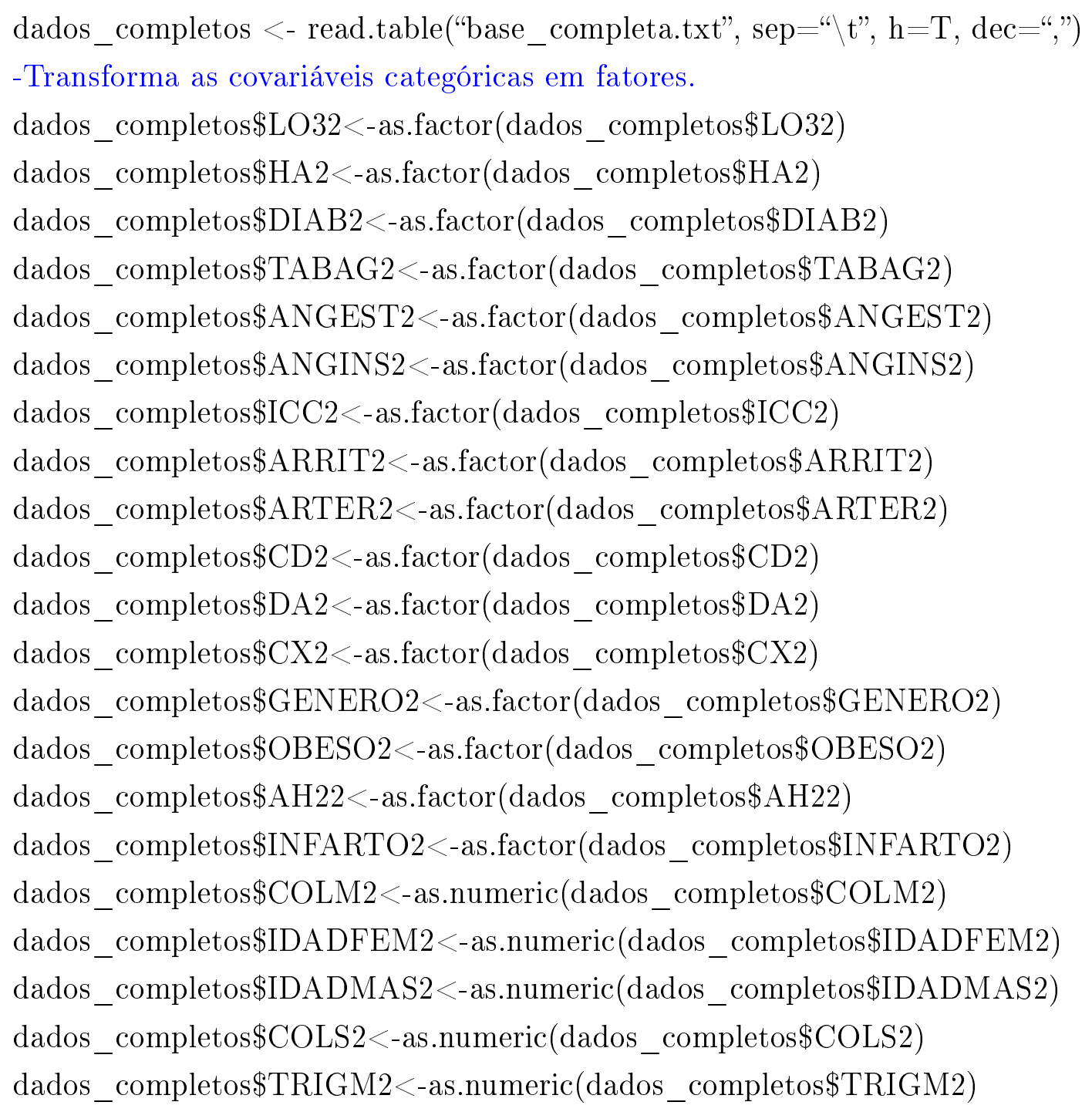


dados_completos $\$$ TRIGS2 <-as.numeric(dados_completos\$TRIGS2)

dados_completos $\$$ GLICM2 $<$-as.numeric(dados_completos $\$$ GLICM2)

dados_completos $\$$ GLICS2<-as.numeric(dados_completos $\$$ GLICS2)

$\operatorname{attach}($ dados_completos)

-Regressão Logística Usual-

-Ajuste dos modelos univariados.

mod_HA2 <-glm(LO32 HA2, family=binomial $)$

mod_DIAB2 $<-\operatorname{glm}($ LO32 $\sim$ DIAB2, family=binomial $)$

mod_TABAG2 <- glm(LO32 TABAG2, family=binomial $)$

mod_ANGEST2 $<-$ glm(LO32 $\sim$ ANGEST2, family=binomial $)$

mod_ANGINS2 <-glm(LO32 ANGINS2, family=binomial $)$

mod_ICC2 $<-$ glm(LO32 ICC2, family=binomial $)$

mod_ARRIT2 $<-\operatorname{glm}($ LO32 $\sim$ ARRIT2, family=binomial $)$

mod_ARTER2 $<-\operatorname{glm}($ LO32 $\sim$ ARTER2, family=binomial $)$

mod_CD2 <-glm(LO32 CD2, family=binomial $)$

mod_DA2 $<-\operatorname{glm}($ LO32 $\sim$ DA2, family=binomial $)$

mod_CX2 $<-\operatorname{glm}($ LO32 $\sim$ CX2, family=binomial $)$

mod_GENERO2 <-glm(LO32 GENERO2, family=binomial)

mod_OBESO2 <- glm(LO32 OBESO2, family=binomial $)$

mod_AH22 <- glm(LO32 AH22, family=binomial $)$

mod_INFARTO2 <-glm(LO32 INFARTO2, family=binomial $)$

mod_COLM2 $<-\operatorname{glm}($ LO32 $\sim$ COLM2, family=binomial $)$

mod_COLS2 $<-\operatorname{glm}($ LO32 $\sim$ COLS2, family=binomial $)$

mod_IDADFEM2 <-glm(LO32 IDADFEM2, family=binomial)

mod_IDADMAS2 $<-\operatorname{glm}($ LO32 $\sim$ IDADMAS2, family=binomial $)$

mod_TRIGM2 $<-\operatorname{glm}($ LO32 $\sim$ TRIGM2, family=binomial $)$

mod_TRIGS2 $<-\operatorname{glm}($ LO32 $\sim$ TRIGS2, family=binomial $)$

mod_GLICM2 <-glm(LO32 GLICM2, family=binomial)

mod_GLICS2 $<-$ glm(LO32 GLICS2, family=binomial)

-Ajuste do modelo somente com as covariáveis significativas a 0,0001.

mod_completo_signif $<-$ glm(LO32 DIAB2 + TABAG2 + ANGEST2 + ANGINS2 +

ARRIT2 + GENERO2 + INFARTO2 + COLM2 + IDADFEM2 + IDADMAS2 + TRIGM2

+ GLICM2, family=binomial, $\mathrm{x}=$ TRUE)

-Base com valores não nulos-

-Leitura dos dados sem informação faltante.

dados_sem_null<-read.table("base_completa_sem_null.txt", sep=" $\backslash \mathrm{t}$ ", $\mathrm{h}=\mathrm{T}$, dec=",") 
-Transforma as covariáveis categóricas em fatores.

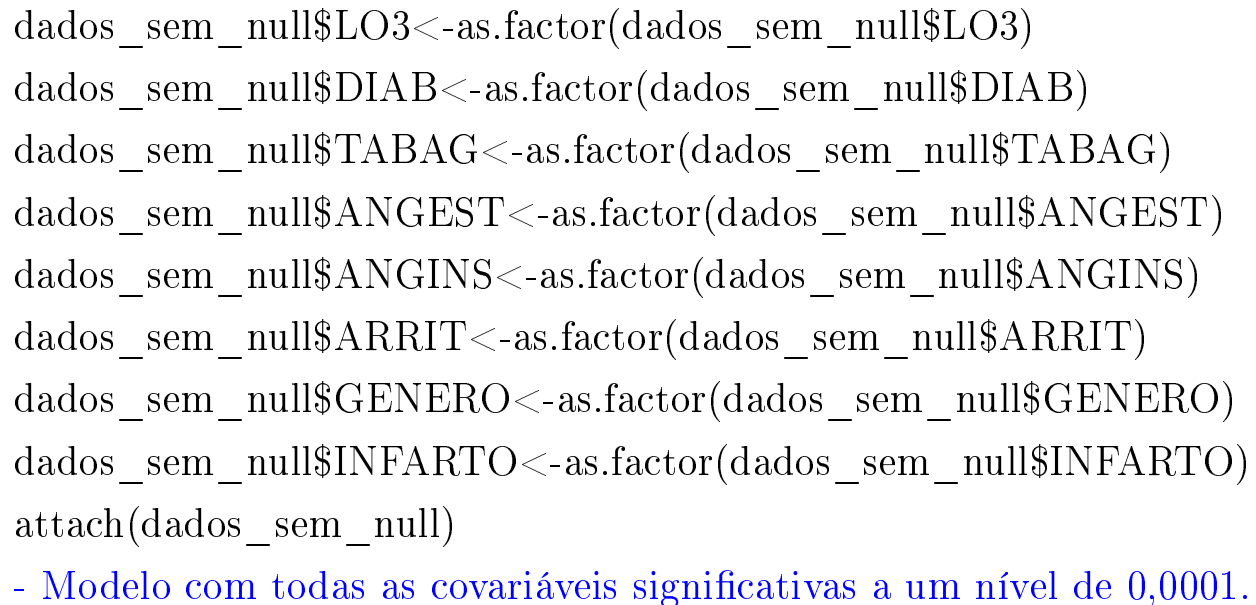

modelo $<-$ glm $(\mathrm{LO} 3 \sim \mathrm{DIAB}+\mathrm{TABAG}+\mathrm{ANGEST}+\mathrm{ANGINS}+\mathrm{ARRIT}+\mathrm{GENERO}+$ INFARTO + COLM + IDADFEM + IDADMAS + TRIGM + GLICM, family=binomial, $\mathrm{x}=\mathrm{TRUE})$

-Retorna os decis das probabilidades estimadas.

quantile(fitted(modelo), probs $=\mathrm{c}(.10, .20, .30, .40, .50, .60, .70, .80, .90))$

-Cria os 10 grupos para cálculo da estatística de Hosmer-Lemeshow.

faixa_emv $<-\operatorname{rep}(0,1025)$

for (i in 1:1025) \{

if(fitted(modelo) $[\mathrm{i}]<=0.3145546)$ faixa_emv[i] $=1$

if(fitted(modelo) $[\mathrm{i}]<=0.4502876 \&$ fitted(modelo) $[\mathrm{i}]>0.3145546)$ faixa_emv[i] $=2$

if(fitted(modelo) $[\mathrm{i}]<=0.5398423 \&$ fitted(modelo) $[\mathrm{i}]>0.4502876)$ faixa_emv $[\mathrm{i}]=3$

if(fitted(modelo) $[\mathrm{i}]<=0.6507115 \&$ fitted(modelo) $[\mathrm{i}]>0.5398423)$ faixa_emv $[\mathrm{i}]=4$

if(fitted(modelo) $[\mathrm{i}]<=0.7441986 \&$ fitted(modelo) $[\mathrm{i}]>0.6507115)$ faixa_emv[i] $=5$

if(fitted(modelo)[i] $<=0.8370346 \&$ fitted(modelo)[i] $>0.7441986)$ faixa_emv $[\mathrm{i}]=6$

if(fitted(modelo)[i] $<=0.9038575 \&$ fitted(modelo) $[\mathrm{i}]>0.8370346)$ faixa_emv[i] $=7$

if(fitted(modelo) $[\mathrm{i}]<=0.9472844 \&$ fitted(modelo) $[\mathrm{i}]>0.9038575)$ faixa_emv[i] $=8$

if(fitted(modelo)[i] $<=0.9766966 \&$ fitted(modelo) $[\mathrm{i}]>0.9472844)$ faixa_emv[i] $=9$

if(fitted(modelo) $[\mathrm{i}]>0.9766966)$ faixa_emv $[\mathrm{i}]=10\}$

table(faixa_emv,LO3)

-Identificação da Multicolinearidade-

-Decomposição em Valores Singulares.

sqrt_w $<-$ diag(sqrt(modelo\$weights))

$\mathrm{k}<-$ sqrt_w $\% * \%$ modelo $\$ \mathrm{x}$

svd_k $<-\operatorname{svd}(\mathrm{k})$

-Índice de Condição.

indice_condicao $<-\max \left(\mathrm{svd} \_\mathrm{k} \$ \mathrm{~d}\right) / \min \left(\mathrm{svd} \_\mathrm{k} \$ \mathrm{~d}\right)$

-Número de Condição. 
svd_k $2<-\operatorname{svd}(\mathrm{t}(\mathrm{k}) \% * \% \mathrm{k})$

numero_condicao $<-\left(\max \left(\mathrm{svd} \_\mathrm{k} 2 \$ \mathrm{~d}\right)\right) /\left(\min \left(\mathrm{svd} \_\mathrm{k} 2 \$ \mathrm{~d}\right)\right)$

-Decomposição da Variância.

prop_var $<-\operatorname{matrix}\left(0\right.$, nrow $=$ nrow $($ svd_k $\$ \mathrm{v})$, ncol $=$ ncol $\left.\left(\mathrm{svd}_{-} \mathrm{k} \$ \mathrm{v}\right)\right)$

colnames(prop_var) <- list("Prop_Var_b0", "Prop_Var_b1", "Prop_Var_b2", "Prop_Var_b3",

"Prop_Var_b4", "Prop_Var_b5", "Prop_Var_b6", "Prop_Var_b7", "Prop_Var_b8",

"Prop_Var_b9", "Prop_Var_b10", "Prop_Var_b11", "Prop_Var_b12")

rownames(prop_var) <- list(svd_k $\$ \mathrm{~d}[1], \mathrm{svd}_{-} \mathrm{k} \$ \mathrm{~d}[2], \mathrm{svd} \_\mathrm{k} \$ \mathrm{~d}[3], \mathrm{svd} \_\mathrm{k} \$ \mathrm{~d}[4], \mathrm{svd} \_\mathrm{k} \$ \mathrm{~d}[5]$,

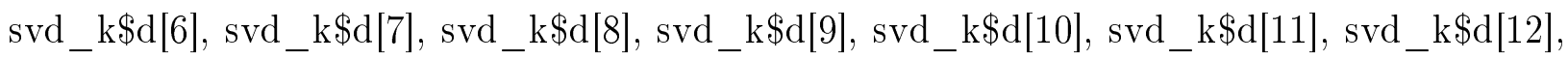
svd_k $\$ d[13])$

$\mathrm{w}<-\operatorname{diag}($ modelo $\$$ weights $)$

covar_beta $<-$ solve $(\mathrm{t}($ modelo $\$ \mathrm{x}) \% * \% \mathrm{w} \% * \%$ modelo $\$ \mathrm{x})$

for(i in 1:nrow(svd_k $\$ \mathrm{v}))\{$

for $\left(\mathrm{j}\right.$ in $\left.1: \operatorname{ncol}\left(\mathrm{svd} \_\mathrm{k} \$ \mathrm{v}\right)\right)\{$

prop_var[j,i]<- $\left(\left(\operatorname{svd} \_\mathrm{k} \$ \mathrm{v}[\mathrm{i}, \mathrm{j}] \wedge 2\right) /\left(\operatorname{svd} \_\mathrm{k} \$ \mathrm{~d}[\mathrm{j}] \wedge 2\right)\right) /($ covar_beta $\left.\left.[\mathrm{i}, \mathrm{i}])\right\}\right\}$

-Coeficiente de Determinação.

-É necessário ler novamente a base, pois os fatores devem ser lidos como variáveis numéricas quando servirem de variável dependente.

dados $2<$-read.table("base_completa_padronizada_sem_null.txt", sep=" \t", h=T, dec=",") mod_DIAB $<-\operatorname{lm}(\operatorname{dad} \operatorname{dos} 2 \$ \mathrm{DIAB} \sim$ factor$(\operatorname{dados} 2 \$ \mathrm{TABAG})+$ factor(dados 2 ANGEST) + factor(dados $2 \$ A N G I N S)+$ factor(dados $2 \$ A R R I T)+$ factor(dados $2 \$$ GENERO $)+$ factor(dados 2 INFARTO) + dados 2 IDADFEM + dados2\$IDADMAS + dados 2 COLM + dados $2 \$$ TRIGM + dados $2 \$$ GLICM)

mod_TABAG $<-\operatorname{lm}($ dados $2 \$$ TABAG $\sim$ factor $($ dados $2 \$$ DIAB $)+$ factor $($ dados $2 \$ A N G E S T)$ + factor(dados $2 \$$ ANGINS $)+$ factor(dados $2 \$$ ARRIT $)+$ factor(dados $2 \$$ GENERO $)+$ factor(dados 2 INFARTO) + dados 2 IDADFEM + dados $2 \$$ IDADMAS + dados 2 COLM + dados $2 \$$ TRIGM + dados $2 \$$ GLICM)

mod_ANGEST $<-\operatorname{lm}($ dados $2 \$ A N G E S T \sim$ factor $($ dados $2 \$ D I A B)+$ factor $($ dados $2 \$ T A B A G)$ + factor(dados $2 \$ A N G I N S)+$ factor(dados $2 \$ A R R I T)+$ factor(dados $2 \$$ GENERO $)+$ factor(dados 2 INFARTO) + dados 2 IDADFEM + dados 2 IDADMAS + dados 2 COLM + dados $2 \$$ TRIGM + dados $2 \$$ GLICM)

mod_ANGINS $<-\operatorname{lm}($ dados $2 \$$ ANGINS $\sim$ factor $($ dados $2 \$ D I A B)+$ factor $($ dados $2 \$$ TABAG $)$ + factor(dados 2 ANGEST) + factor(dados $2 \$ A R R I T)+$ factor(dados $2 \$$ GENERO $)+$ factor(dados $2 \$ I N F A R T O)+$ dados $2 \$$ IDADFEM + dados $2 \$ I D A D M A S+\operatorname{dados} 2 \$ C O L M+$ dados $2 \$$ TRIGM + dados $2 \$$ GLICM)

mod_ARRIT $<-\operatorname{lm}($ dados $2 \$ A R R I T \sim$ factor $($ dados $2 \$ D I A B)+$ factor $($ dados $2 \$$ TABAG $)+$ factor(dados $2 \$ A N G E S T)+$ factor(dados $2 \$ A N G I N S)+$ factor(dados $2 \$$ GENERO $)+$ factor(dados 2 INFARTO) + dados 2 IDADFEM + dados $2 \$$ IDADMAS + dados $2 \$ C O L M+$ dados $2 \$$ TRIGM + dados $2 \$$ GLICM)

mod_GENERO $<-\operatorname{lm}($ dados $2 \$$ GENERO $\sim$ factor $($ dados $2 \$$ DIAB $)+$ factor $($ dados $2 \$$ TABAG $)$ 
+ factor(dados $2 \$ A N G E S T)+$ factor(dados $2 \$ A N G I N S)+$ factor(dados $2 \$ A R R I T)+$ factor(dados $2 \$$ INFARTO $)+$ dados $2 \$ I D A D F E M+$ dados $2 \$ I D A D M A S+\operatorname{dados} 2 \$ C O L M+$ dados $2 \$$ TRIGM + dados 2 GLICM)

mod_INFARTO $<-\operatorname{lm}($ dados $2 \$$ INFARTO $\sim$ factor $($ dados $2 \$ D I A B)+$ factor $($ dados $2 \$$ TABAG $)$ + factor(dados $2 \$ A N G E S T)+$ factor(dados $2 \$ A N G I N S)+$ factor(dados $2 \$ A R R I T)+$ factor(dados 2 GENERO) + dados 2 IDADFEM + dados 2 IDADMAS + dados 2 COLM + da$\operatorname{dos} 2 \$$ TRIGM $+\operatorname{dados} 2 \$$ GLICM)

mod_IDADFEM $<-\operatorname{lm}($ dados $2 \$ I D A D F E M ~ \sim ~$ factor $($ dados $2 \$ D I A B)+$ factor $($ dados $2 \$ T A B A G)$ + factor(dados $2 \$ A N G E S T)+$ factor(dados $2 \$ A N G I N S)+$ factor(dados $2 \$ A R R I T)+$ factor $($ dados $2 \$$ GENERO $)+$ factor $($ dados $2 \$$ INFARTO $)+$ dados $2 \$$ IDADMAS + dados $2 \$$ COLM + dados $2 \$ T R I G M+$ dados $2 \$$ GLICM) mod_IDADMAS $<-\operatorname{lm}($ dados $2 \$$ IDADMAS $\sim$ factor $($ dados $2 \$ D I A B)+$ factor $($ dados $2 \$$ TABAG $)$ + factor(dados $2 \$ A N G E S T)+$ factor(dados $2 \$ A N G I N S)+$ factor(dados $2 \$ A R R I T)+$ factor $($ dados $2 \$$ GENERO $)+$ factor $($ dados 2 INFARTO $)+$ dados 2 IDADFEM + dados 2 COLM + dados $2 \$$ TRIGM + dados $2 \$$ GLICM) mod_COLM $<-\operatorname{lm}($ dados $2 \$ C O L M \sim$ factor $($ dados $2 \$ D I A B)+$ factor $($ dados $2 \$$ TABAG $)+$ factor(dados $2 \$$ ANGEST) + factor(dados $2 \$ A N G I N S)+$ factor(dados $2 \$ A R R I T)+$ factor(dados $2 \$$ GENERO) + factor(dados $2 \$$ INFARTO $)+$ dados $2 \$$ IDADFEM + dados 2 IDADMAS + dados 2 TRIGM + dados $2 \$$ GLICM) mod_TRIGM $<-\operatorname{lm}($ dados $2 \$$ TRIGM $\sim$ factor(dados $2 \$ D I A B)+$ factor(dados $2 \$$ TABAG) + factor(dados $2 \$ A N G E S T)+$ factor(dados 2 ANGINS $)+$ factor(dados 2 ARRIT $)+$ factor $($ dados $2 \$$ GENERO $)+$ factor $($ dados $2 \$$ INFARTO $)+$ dados $2 \$ I D A D F E M+$ dados 2 IDADMAS + dados $2 \$$ COLM + dados $2 \$$ GLICM) mod_GLICM $<-\operatorname{lm}($ dados $2 \$$ GLICM $\sim$ factor(dados $2 \$ D I A B)+$ factor(dados $2 \$$ TABAG) + factor(dados $2 \$ A N G E S T)+$ factor(dados $2 \$ A N G I N S)+$ factor(dados $2 \$ A R R I T)+$ factor (dados $2 \$$ GENERO) + factor(dados 2 INFARTO $)+$ dados 2 IDADFEM + dados 2 IDADMAS + dados $2 \$$ COLM + dados $2 \$$ TRIGM)

-Retorna uma tabela com os valores de R2.

library (SMIR)

matriz_R $2<-\operatorname{matrix}(0$, nrow $=12$, ncol $=2)$

colnames(matriz_R2) <- list("Covariavel","R2")

matriz_R2[1,] <- cbind("DIAB",R2(mod_DIAB))

matriz_R2[2,] <- cbind("TABAG",R2(mod_TABAG))

matriz_R2[3,] <- cbind(“ANGEST",R2(mod_ANGEST))

matriz_R2[4,] <- cbind("ANGINS",R2(mod_ANGINS))

matriz_R2[5,]<- cbind("ARRIT",R2(mod_ARRIT))

matriz_R2[6,] <- cbind(“GENERO",R2(mod_GENERO))

matriz_R2[7,] <- cbind("INFARTO",R2(mod_INFARTO))

matriz_R2[8,] <- cbind("IDADFEM",R2(mod_IDADFEM))

matriz_R2[9,] <- cbind("IDADMAS",R2(mod_IDADMAS)) 
matriz_R2[10,] <- cbind("COLM",R2(mod_COLM))

matriz_R2[11,] <- cbind("TRIGM",R2(mod_TRIGM))

matriz_R2[12,] <- cbind(“GLICM",R2(mod_GLICM))

-Regressão em Cristas-

- Varáveis auxiliares para cálculo dos lambdas.

dados $2 \$$ LO3 <- as.numeric(dados $2 \$$ LO3)

vetor_resp <-as.vector(dados $2 \$$ LO3)

s2 <- (t(vetor_resp - modelo $\$$ fitted.values) $\% * \%$ (vetor_resp -

modelo\$fitted.values $)) /\left(\operatorname{nrow}(\operatorname{modelo} \$ \mathrm{x})-\operatorname{ncol}\left(\operatorname{svd} \_\mathrm{k} \$ \mathrm{v}\right)-1\right)$

alpha_prod $<-1$

alpha $<-\operatorname{rep}(0$, ncol$($ svd_k $\$ v))$

$\mathrm{m} 2<-\operatorname{rep}\left(0, \mathrm{ncol}\left(\mathrm{svd} \_\mathrm{k} \$ \mathrm{v}\right)\right)$

for (i in 1:ncol(svd_k $\$ \mathrm{v}))\{$

alpha_i $<-$ svd_k $\$ v[i] \% * \$,$% modelo \$$ coefficients

alpha[i] $<-$ alpha_i

alpha_prod $<-$ alpha_prod $*(($ alpha_i $) \wedge 2)$

$\mathrm{m} 2[\mathrm{i}]<-\mathrm{s} 2 /(($ alpha_i $) \wedge 2)\}$

vetor_s $<-$ rep $\left(0, n c o l\left(s v d \_k \$ v\right)\right)$

for (i in 1:ncol(svd_k $\$ \mathrm{v}))\{$

vetor_s $[\mathrm{i}]<-\left(\operatorname{svd} \_\mathrm{k} \$ \mathrm{~d}[\mathrm{i}] * \mathrm{~s} 2\right) /\left(\left(\left(\operatorname{nrow}(\operatorname{modelo} \$ \mathrm{x})-\mathrm{ncol}\left(\mathrm{svd} \_\mathrm{k} \$ \mathrm{v}\right)-1\right) * \mathrm{~s} 2\right)+\left(\mathrm{svd} \_\mathrm{k} \$ \mathrm{~d}[\mathrm{i}]\right.\right.$

$*(($ svd_k $\$ \mathrm{v}[\mathrm{i}] \% * \$,$% modelo \$$ coefficients $) \wedge 2)))\}$

-Calcula os valores dos lambdas.

lambda_1<-s2/max(alpha)

lambda_2 $<-\operatorname{ncol}\left(\mathrm{svd} \_\mathrm{k} \$ \mathrm{v}\right) /(\mathrm{t}($ modelo $\$$ coefficients $) \% * \%$ modelo $\$$ coefficients $)$

lambda_3<-s $2 /\left((\right.$ alpha_prod $\left.) \wedge\left(1 / \operatorname{ncol}\left(\operatorname{svd} \_\mathrm{k} \$ \mathrm{v}\right)\right)\right)$

lambda_4 $<-$ median $(\mathrm{m} 2)$

lambda_5 $<-\max ($ vetor_s)

-Calcula as estimativas dos coeficientes e das variâncias dos coeficientes dos modelos.

xwx $<-\mathrm{t}($ modelo $\$ \mathrm{x}) \% * \% \operatorname{diag}($ modelo $\$$ weights $) \% * \%$ modelo $\$ \mathrm{x}$

-Lambda 1.

diag_lambda1<-diag(rep(lambda_1,13),13)

beta_lambda $1<-$ solve $(\mathrm{xwx}+$ diag_lambda1) $\% * \%(\mathrm{xwx}) \% * \%$ modelo $\$$ coefficients

var_beta_lambda1 <-solve(xwx + diag_lambda1)\%*\%(xwx)\%*\%solve(xwx + diag_lambda1)

soma_ridge $1<-\operatorname{rep}(0,1025)$

pred_ridge $<<-\operatorname{rep}(0,1025)$

for (i in 1:1025)\{

soma_ridge1[i] <- beta_lambda1[1] + (dados2\$DIAB[i] * beta_lambda1[2]) + (dados2\$TABAG[i]

* beta_lambda1[3] $)+($ dados 2 \$ANGEST[i]* beta_lambda1[4] $)+($ dados 2 \$ANGINS[i] 
* beta_lambda1[5] $)+($ dados 2 \$ARRIT[i] * beta_lambda1[6] $)+($ dados 2 GENERO[i]

* beta_lambda1[7] $)+($ dados 2 INFARTO[i] * beta_lambda1[8] $)+($ dados $2 \$ C O L M[i]$

* beta_lambda1[9] $)+($ dados2\$IDADFEM[i] * beta_lambda1[10] $)+($ dados2\$IDADMAS[i]

* beta_lambda1[11] $)+($ dados2\$TRIGM[i] * beta_lambda1[12] $)+($ dados $2 \$$ GLICM[i]

* beta_lambda1[13])

pred_ridge1[i] <-exp $($ soma_ridge1[i] $) /(1+\exp ($ soma_ridge1[i] $))\}$

-Lambda 2.

diag_lambda2 <-diag(rep(lambda_2,13),13)

beta_lambda $2<-$ solve $(\mathrm{xwx}+$ diag_lambda 2$) \% * \%(\mathrm{xwx}) \% * \%$ modelo $\$$ coefficients

var_beta_lambda $2<-$ solve $(x w x+$ diag_lambda2 $) \% * \%(x w x) \% * \%$ solve $(x w x+$ diag_lambda2)

soma_ridge $2<-\operatorname{rep}(0,1025)$

pred_ridge $2<-\operatorname{rep}(0,1025)$

for (i in 1:1025) \{

soma_ridge2[i] <- beta_lambda2[1] + (dados $2 \$ D I A B[i] *$ beta_lambda2[2] $)+($ dados $2 \$ T A B A G[i]$

$*$ beta_lambda2[3] $)+($ dados 2 \$ANGEST[i] $*$ beta_lambda2[4] $)+(\operatorname{dados} 2 \$$ ANGINS[i]

* beta_lambda2[5]) + (dados2\$ARRIT[i] * beta_lambda2[6] $)+($ dados 2 GENERO[i]

* beta_lambda2[7]) + (dados2\$INFARTO[i] * beta_lambda2[8]) + (dados2\$COLM[i]

* beta_lambda2[9] $)+($ dados2\$IDADFEM[i] * beta_lambda2[10] $)+($ dados2\$IDADMAS[i]

* beta_lambda2[11] $)+($ dados2 $\$$ TRIGM[i] * beta_lambda2[12] $)+($ dados $2 \$$ GLICM[i]

* beta_lambda2[13])

pred_ridge $2[\mathrm{i}]<-\exp ($ soma_ridge $2[\mathrm{i}]) /(1+\exp ($ soma_ridge $2[\mathrm{i}]))\}$

-Lambda 3.

diag_lambda3<-diag(rep(lambda_3,13),13)

beta_lambda3 <-solve(xwx + diag_lambda 3$) \% * \%(x w x) \% * \%$ modelo $\$$ coefficients

var_beta_lambda3 $<-$ solve $(\mathrm{xwx}+$ diag_lambda 3$) \% * \%(\mathrm{xwx}) \% * \%$ solve $(\mathrm{xwx}+$ diag_lambda3 $)$

soma_ridge $3<-$ rep $(0,1025)$

pred_ridge $3<-\operatorname{rep}(0,1025)$

for (i in 1:1025) \{

soma_ridge3[i] <- beta_lambda3[1] + (dados2\$DIAB[i] * beta_lambda3[2] $)+($ dados 2 TABAG[i]

* beta_lambda3[3]) + (dados 2 \$ANGEST[i]* beta_lambda3[4] $)+($ dados2\$ANGINS[i]

* beta_lambda3[5]) + (dados2\$ARRIT[i] * beta_lambda3[6]) + (dados2\$GENERO[i]

* beta_lambda3[7]) + (dados2\$INFARTO[i] * beta_lambda3[8]) + (dados2\$COLM[i]

* beta_lambda3[9]) + (dados2\$IDADFEM[i] * beta_lambda3[10]) + (dados2\$IDADMAS[i]

* beta_lambda3[11]) + (dados2\$TRIGM[i] * beta_lambda3[12]) + (dados2\$GLICM[i]

* beta_lambda3[13])

pred_ridge $3[\mathrm{i}]<-\exp ($ soma_ridge $3[\mathrm{i}]) /(1+\exp ($ soma_ridge $3[\mathrm{i}]))\}$

-Lambda 4.

diag_lambda4 <-diag(rep(lambda_4,13),13)

beta_lambda $4<-$ solve(xwx + diag_lambda 4$) \% * \%(\mathrm{xwx}) \% * \%$ modelo $\$$ coefficients

var_beta_lambda $4<-$ solve $(\mathrm{xwx}+\operatorname{diag}$ _lambda 4$) \% * \%(\mathrm{xwx}) \% * \%$ solve $(\mathrm{xwx}+$ diag_lambda4 $)$ 
soma_ridge $4<-\operatorname{rep}(0,1025)$

pred_ridge $4<-\operatorname{rep}(0,1025)$

for (i in 1:1025) \{

soma_ridge4[i] <- beta_lambda4[1] + (dados2\$DIAB[i] * beta_lambda4[2]) + (dados2\$TABAG[i]

* beta_lambda4[3] $)+($ dados $2 \$ A N G E S T[i] *$ beta_lambda4[4] $)+($ dados $2 \$ A N G I N S[i]$

* beta_lambda4[5]) + (dados 2 ARRIT[i] * beta_lambda4[6]) + (dados 2 SGENERO[i]

* beta_lambda4[7]) + (dados2\$INFARTO[i] * beta_lambda4[8]) + (dados2\$COLM[i]

* beta_lambda4[9]) + (dados2\$IDADFEM[i] * beta_lambda4[10]) + (dados2\$IDADMAS[i]

* beta_lambda4[11] $)+(\operatorname{dados} 2 \$$ TRIGM[i] * beta_lambda4[12] $)+(\operatorname{dados} 2 \$$ GLICM[i]

* beta_lambda4[13])

pred_ridge $4[\mathrm{i}]<-\exp ($ soma_ridge $4[\mathrm{i}]) /(1+\exp ($ soma_ridge $4[\mathrm{i}]))\}$

-Lambda 5.

diag_lambda5<-diag(rep(lambda_5,13),13)

beta_lambda $5<-$ solve $(\mathrm{xwx}+$ diag_lambda 5$) \% * \%(\mathrm{xwx}) \% * \%$ modelo $\$$ coefficients

var_beta_lambda $5<-$ solve $(\mathrm{xwx}+$ diag_lambda5) $\% * \%(\mathrm{xwx}) \% * \%$ solve(xwx + diag_lambda5)

soma_ridge $5<-\operatorname{rep}(0,1025)$

pred_ridge $<-\operatorname{rep}(0,1025)$

for (i in 1:1025)\{

soma_ridge $5[\mathrm{i}]<-$ beta_lambda5[1] $+($ dados $2 \$ D I A B[i] *$ beta_lambda5[2] $)+($ dados 2 TABAG[i]

* beta_lambda5[3]) + (dados 2 \$ANGEST[i]* beta_lambda5[4]) + (dados2\$ANGINS[i]

* beta_lambda5[5] $)+($ dados $2 \$$ ARRIT[i] * beta_lambda5[6] $)+($ dados 2 GENERO[i]

* beta_lambda5[7]) + (dados2\$INFARTO[i] * beta_lambda5[8]) + (dados2\$COLM[i]

* beta_lambda5[9] $)+($ dados 2 IDADFEM[i] * beta_lambda5[10]) + (dados2\$IDADMAS[i]

* beta_lambda5[11] $)+($ dados $2 \$$ TRIGM $[\mathrm{i}] *$ beta_lambda5[12] $)+($ dados 2 SLICM[i]

* beta_lambda5[13])

pred_ridge $5[\mathrm{i}]<-\exp ($ soma_ridge $5[\mathrm{i}]) /(1+\exp ($ soma_ridge $5[\mathrm{i}]))\}$

-Retorna os decis das probabilidades estimadas pelos modelos.

quantile(pred_ridge1, probs $=\mathrm{c}(.10, .20, .30, .40, .50, .60, .70, .80, .90))$

quantile(pred_ridge2, probs $=\mathrm{c}(.10, .20, .30, .40, .50, .60, .70, .80, .90))$

quantile(pred_ridge 3, probs $=\mathrm{c}(.10, .20, .30, .40, .50, .60, .70, .80, .90))$

quantile(pred_ridge 4, probs $=\mathrm{c}(.10, .20, .30, .40, .50, .60, .70, .80, .90))$

quantile(pred_ridge 5, probs $=\mathrm{c}(.10, .20, .30, .40, .50, .60, .70, .80, .90))$

-Cria os grupos para cada modelo para cálculo da estatística de Hosmer-Lemeshow.

faixa_1 $<-$ rep $(0,1025)$

for ( $\mathrm{i}$ in $1: 1025)\{$

if(pred_ridge1 $[\mathrm{i}]<=0.3172762$ ) faixa_1 $[\mathrm{i}]=1$

if(pred_ridge1 $[\mathrm{i}]<=0.4510182 \&$ pred_ridge1 $[\mathrm{i}]>0.3172762)$ faixa_1[i] $=2$

if(pred_ridge1 $[\mathrm{i}]<=0.5410136 \&$ pred_ridge1 $[\mathrm{i}]>0.4510182)$ faixa_1[i] $=3$

if(pred_ridge1 $[\mathrm{i}]<=0.6513671 \&$ pred_ridge1 $[\mathrm{i}]>0.5410136)$ faixa_1 $[\mathrm{i}]=4$

if(pred_ridge1 $[\mathrm{i}]<=0.7437074 \&$ pred_ridge1 $[\mathrm{i}]>0.6513671)$ faixa_1[i] $=5$ 
if(pred_ridge1[i] $<=0.8353081 \&$ pred_ridge1[i] $>0.7437074)$ faixa_1 $[\mathrm{i}]=6$ if(pred_ridge1[i] $<=0.9029298 \&$ pred_ridge1[i] $>0.8353081)$ faixa_1 $[\mathrm{i}]=7$ if(pred_ridge1[i] $<=0.9462491 \&$ pred_ridge1[i] $>0.9029298)$ faixa_1[i] $=8$ if $($ pred_ridge1 $[\mathrm{i}]<=0.975841 \&$ pred_ridge1[i] $>0.9462491)$ faixa_1[i] $=9$ if $($ pred_ridge1 $[\mathrm{i}]>0.975841)$ faixa_1 $[\mathrm{i}]=10\}$

faixa_2 <-rep $(0,1025)$

for ( $\mathrm{i}$ in $1: 1025)\{$

if(pred_ridge2 $[\mathrm{i}]<=0.3302818$ ) faixa_2[i] $=1$

if(pred_ridge2[i] $<=0.4571444 \&$ pred_ridge2[i] $>0.3302818)$ faixa_2[i] $=2$ if(pred_ridge2 $[\mathrm{i}]<=0.5494281 \&$ pred_ridge2 $[\mathrm{i}]>0.4571444$ ) faixa_2 $[\mathrm{i}]=3$ if(pred_ridge2 $[\mathrm{i}]<=0.6529506 \&$ pred_ridge2 $[\mathrm{i}]>0.5494281$ ) faixa_2[i] $=4$ if(pred_ridge $2[\mathrm{i}]<=0.7389097 \&$ pred_ridge $2[\mathrm{i}]>0.6529506)$ faixa_2 $[\mathrm{i}]=5$ if(pred_ridge2[i] $<=0.8262699 \&$ pred_ridge $2[\mathrm{i}]>0.7389097)$ faixa_2 $[\mathrm{i}]=6$ if(pred_ridge2 $[\mathrm{i}]<=0.8972824 \&$ pred_ridge2 $[\mathrm{i}]>0.8262699)$ faixa_2 $[\mathrm{i}]=7$ if(pred_ridge2 $[\mathrm{i}]<=0.9391859 \&$ pred_ridge2 $[\mathrm{i}]>0.8972824$ ) faixa_2[i] $=8$ if(pred_ridge $2[\mathrm{i}]<=0.9709301 \&$ pred_ridge $2[\mathrm{i}]>0.9391859)$ faixa_2 $[\mathrm{i}]=9$ if $($ pred_ridge2[i] $>0.9709301)$ faixa_2[i] $=10\}$

faixa_3<-rep $(0,1025)$

for (i in 1:1025) \{

if(pred_ridge $3[\mathrm{i}]<=0.3251481)$ faixa_3[i] $=1$

if(pred_ridge $3[\mathrm{i}]<=0.4567112 \&$ pred_ridge $3[\mathrm{i}]>0.3251481)$ faixa_3[i] $=2$ if(pred_ridge3[i] $<=0.5452436 \&$ pred_ridge3[i] $>0.4567112$ ) faixa_3[i] $=3$ if(pred_ridge $3[\mathrm{i}]<=0.6533266 \&$ pred_ridge $3[\mathrm{i}]>0.5452436$ ) faixa_3[i] $=4$ if(pred_ridge $3[\mathrm{i}]<=0.7392778 \&$ pred_ridge $3[\mathrm{i}]>0.6533266)$ faixa_3[i] $=5$ if(pred_ridge3[i] $<=0.8281497 \&$ pred_ridge3[i] $>0.7392778$ ) faixa_3[i] $=6$ if(pred_ridge3[i] $<=0.8996935 \&$ pred_ridge3[i] $>0.8281497$ ) faixa_3[i] $=7$ if(pred_ridge3[i] $<=0.9420807 \&$ pred_ridge3[i] $>0.8996935)$ faixa_3[i] $=8$ if(pred_ridge3[i] $<=0.972808 \&$ pred_ridge3[i] $>0.9420807)$ faixa_3[i] $=9$ if $($ pred_ridge $3[\mathrm{i}]>0.972808)$ faixa_3 $[\mathrm{i}]=10\}$

faixa_ $4<-\operatorname{rep}(0,1025)$

for (i in 1:1025) \{

if(pred_ridge $4[\mathrm{i}]<=0.3187443)$ faixa_4[i] $=1$

if(pred_ridge4 $[\mathrm{i}]<=0.4515298 \&$ pred_ridge4 $[\mathrm{i}]>0.3187443)$ faixa_4 $[\mathrm{i}]=2$ if(pred_ridge $4[\mathrm{i}]<=0.5416089 \&$ pred_ridge $4[\mathrm{i}]>0.4515298)$ faixa_4[i] $=3$ if(pred_ridge $4[\mathrm{i}]<=0.6519337 \&$ pred_ridge $4[\mathrm{i}]>0.5416089)$ faixa_4[i] $=4$ if(pred_ridge $4[\mathrm{i}]<=0.7420564 \&$ pred_ridge $4[\mathrm{i}]>0.6519337)$ faixa_4 $[\mathrm{i}]=5$ if(pred_ridge4 $[\mathrm{i}]<=0.8337053 \&$ pred_ridge4 $[\mathrm{i}]>0.7420564$ ) faixa_4[i] $=6$ if(pred_ridge4 $[\mathrm{i}]<=0.9023817 \&$ pred_ridge4 $[\mathrm{i}]>0.8337053)$ faixa_4 $[\mathrm{i}]=7$ if(pred_ridge $4[\mathrm{i}]<=0.9455439 \&$ pred_ridge $4[\mathrm{i}]>0.9023817)$ faixa_4 $[\mathrm{i}]=8$ if(pred_ridge $4[\mathrm{i}]<=0.9752908 \&$ pred_ridge $4[\mathrm{i}]>0.9455439)$ faixa_4 $[\mathrm{i}]=9$ 
if $($ pred_ridge $4[\mathrm{i}]>0.9752908)$ faixa_4 $[\mathrm{i}]=10\}$

faixa_5 <-rep $(0,1025)$

for ( $\mathrm{i}$ in $1: 1025)\{$

if(pred_ridge $5[\mathrm{i}]<=0.3151204$ ) faixa_5[i] $=1$

if(pred_ridge $5[\mathrm{i}]<=0.4507414 \&$ pred_ridge $5[\mathrm{i}]>0.3151204)$ faixa_5[i] $=2$

if(pred_ridge $5[\mathrm{i}]<=0.5398817 \&$ pred_ridge $5[\mathrm{i}]>0.4507414)$ faixa_5[i] $=3$

if(pred_ridge $5[\mathrm{i}]<=0.6506465 \&$ pred_ridge $5[\mathrm{i}]>0.5398817)$ faixa_5[i] $=4$

if(pred_ridge $5[\mathrm{i}]<=0.744139 \&$ pred_ridge $5[\mathrm{i}]>0.6506465)$ faixa_5[i] $=5$

if(pred_ridge $5[\mathrm{i}]<=0.8367881 \&$ pred_ridge $5[\mathrm{i}]>0.744139)$ faixa_5[i] $=6$

if(pred_ridge $5[\mathrm{i}]<=0.90362 \&$ pred_ridge $5[\mathrm{i}]>0.8367881)$ faixa_5[i] $=7$

if(pred_ridge $5[\mathrm{i}]<=0.9471136 \&$ pred_ridge $5[\mathrm{i}]>0.90362)$ faixa_5[i] $=8$

if(pred_ridge $5[\mathrm{i}]<=0.9765429 \&$ pred_ridge $5[\mathrm{i}]>0.9471136)$ faixa_5[i] $=9$

if $($ pred_ridge $5[\mathrm{i}]>0.9765429)$ faixa_5[i] $=10\}$

-Análise de Componentes Principais-

-Conta quantos índices de condição são maiores do que 10.

cont $<-0$

for $\left(\mathrm{m}\right.$ in 1:length $\left.\left(\mathrm{svd} \_\mathrm{k} \$ \mathrm{~d}\right)\right)\{$

if $\left(\left(\max \left(\mathrm{svd}_{-} \mathrm{k} \$ \mathrm{~d}\right) / \mathrm{svd} \_\mathrm{k} \$ \mathrm{~d}[\mathrm{~m}]\right)>10\right)$ cont $<-$ cont +1$\}$

-Passo intermediário para calcular a probabilidade predita.

autovetores_q<-svd_k $\$ \mathrm{v}\left[, 1:\left(\right.\right.$ length $\left(\mathrm{svd} \_\mathrm{k} \$ \mathrm{~d}\right)$-cont $\left.)\right]$

autovalores_2 $<-\left(\operatorname{svd} \_\mathrm{k} \$ \mathrm{~d} \wedge(-2)\right)\left[1:\left(\right.\right.$ length $\left(\mathrm{svd}_{-} \mathrm{k} \$ \mathrm{~d}\right)$-cont $\left.)\right]$

autovalores_q $<-\operatorname{diag}($ autovalores_2,(length(svd_k $\$ \mathrm{~d})$-cont $)$ )

$\mathrm{xwx} \_\mathrm{q}<-$ autovetores_q $\% * \%$ autovalores_q \%*\% t(autovetores_q)

-Calcula o valor da estimativa dos parâmetros e das estimativas das variâncias dos parâmetros.

beta_cp $<-\mathrm{xwx} \_\mathrm{q} \% * \%(\mathrm{t}(\mathrm{k}) \% * \% \mathrm{k}) \% * \%$ modelo $\$$ coefficients

var_beta_cp <- xwx_q $\% * \%(\mathrm{t}(\mathrm{k}) \% * \% \mathrm{k}) \% * \% \mathrm{xwx} \_\mathrm{q}$

-Calcula a probabilidade predita.

soma_cp $<-\operatorname{rep}(0,1025)$

pred_cp $<-\operatorname{rep}(0,1025)$

for ( $\mathrm{i}$ in $1: 1025)\{$

soma_cp[i] <- beta_cp[1] $+($ dados $2 \$ D I A B[i] *$ beta_cp[2] $)+($ dados $2 \$ T A B A G[i] *$ beta_cp[3] $)$

$+($ dados 2 \$ANGEST[i] $*$ beta_cp[4] $)+($ dados 2 \$ANGINS[i] $*$ beta_cp[5] $)+($ dados 2 \$ARRIT[i]

$*$ beta_cp[6] $)+($ dados $2 \$$ GENERO[i] $*$ beta_cp[7] $)+($ dados $2 \$$ INFARTO[i] $*$ beta_cp[8] $)$

$+($ dados $2 \$ C O L M[i] *$ beta_cp $[9])+($ dados 2 \$IDADFEM[i] $*$ beta_cp[10] $)+($ dados 2 SIDADMAS[i]

* beta_cp[11] $)+($ dados $2 \$ T R I G M[i] *$ beta_cp[12] $)+($ dados $2 \$$ GLICM[i] $*$ beta_cp[13] $)$

pred_cp[i] $<-\exp ($ soma_cp[i] $) /(1+\exp ($ soma_cp[i] $))\}$

dp_beta_cp $<-\operatorname{rbind}(\operatorname{sqrt}($ var_beta_cp[1,1]), sqrt(var_beta_cp[2,2]), sqrt(var_beta_cp[3,3]) 
, sqrt(var_beta_cp[4,4]), sqrt(var_beta_cp[5,5]), sqrt(var_beta_cp[6,6]), sqrt(var_beta_cp[7,7])

, sqrt(var_beta_cp[8,8]), sqrt(var_beta_cp[9,9]), sqrt(var_beta_cp[10,10])

, sqrt(var_beta_cp[11,11]), sqrt(var_beta_cp[12,12]), sqrt(var_beta_cp[13,13]))

-Calcula o valor dos decis das probabilidades preditas.

quantile(pred_cp, probs $=\mathrm{c}(.10, .20, .30, .40, .50, .60, .70, .80, .90))$

-Cria os grupos para cálculo da estatística de Hosmer-Lemeshow.

faixa_cp $<-\operatorname{rep}(0,1025)$

for (i in 1:1025) \{

if(pred_cp[i $]<=0.3156312)$ faixa_cp $[\mathrm{i}]=1$

if $($ pred_cp $[\mathrm{i}]<=0.4500416 \&$ pred_cp $[\mathrm{i}]>0.3156312)$ faixa_cp $[\mathrm{i}]=2$

if(pred_cp[i] $<=0.5461903 \&$ pred_cp $[\mathrm{i}]>0.4500416)$ faixa_cp[i] $=3$

if(pred_cp $[\mathrm{i}]<=0.6557416 \&$ pred_cp $[\mathrm{i}]>0.5461903)$ faixa_cp $[\mathrm{i}]=4$

if(pred_cp $[\mathrm{i}]<=0.7476211 \&$ pred_cp $[\mathrm{i}]>0.6557416)$ faixa_cp[i] $=5$

if(pred_cp[i] $<=0.8344489 \&$ pred_cp $[\mathrm{i}]>0.7476211)$ faixa_cp $[\mathrm{i}]=6$

if(pred_cp[i] $<=0.9060274 \&$ pred_cp $[\mathrm{i}]>0.8344489)$ faixa_cp $[\mathrm{i}]=7$

if(pred_cp[i] $<=0.9450451 \&$ pred_cp[i] $>0.9060274)$ faixa_cp $[\mathrm{i}]=8$

if(pred_cp[i] $<=0.9741399 \&$ pred_cp $[\mathrm{i}]>0.9450451)$ faixa_cp[i] $=9$

if(pred_cp[i] $>0.9741399)$ faixa_cp[i] $=10\}$ 
102 APÊNDICE C 


\section{Apêndice D}

\section{Tabelas dos Resultados da Aplicação}

\begin{tabular}{lll}
\hline \hline Variável & Descrição & Categorias \\
\hline HA & Indivíduo apresentou hipertensão arterial & 0 - não; 1 - sim \\
DIAB & Paciente apresentou diabete mellitus & 0 - não; 1 - sim \\
TABAG & Paciente apresentou tabagismo & 0 - não; 1 - sim \\
ANGEST & Paciente apresentou angina estável & 0 - não; 1 - sim \\
ANGINS & Paciente apresentou angina instável & 0 - não; 1 - sim \\
ICC & Paciente apresentou insuficiência cardíaca & 0 - não; 1 - sim \\
ARRIT & Paciente apresentou arritmia & 0 - não; 1 - sim \\
ARTER & Em qual artéria o paciente apresentou & 0 - nenhuma; 1 - carótida \\
& arteriopatia & 2 - aorta; 3 - carótida + aorta \\
CD & Grau da lesão na coronária direita & 0 a 4 \\
DA & Grau da lesão na artéria descendente anterior & 0 a 4 \\
CX & Grau da lesão na artéria circunflexa & 0 a 4 \\
PLAQ & Taxa de plaquetas do paciente $\left(1000 / m m^{3}\right)$ & \\
FIB & Taxa de fibrinogênio do paciente $(m m / \%)$ & \\
GENERO & Gênero do paciente & 0 - fem; 1 - masc \\
LO3 & Grau da LO do paciente & 0 - <50\%; 1 - > =50\% \\
OBESO & Paciente é obeso & 0 - não; 1 - sim \\
AH2 & Presença de antecedentes hereditários & 0 - não; 1 - sim \\
INFARTO & Paciente infartou & 0 - não; 1 - sim \\
C/H & COL / HDL (mg/dl) & \\
L/H & LDL / HDL (mg/dl) & \\
COLM & Taxa de colesterol com medicamento $(\mathrm{mg} / \mathrm{dl})$ & \\
\hline \hline & & \\
\hline
\end{tabular}

Tabela D.1: Descrição das variáveis da base de aplicação. 


\begin{tabular}{lll}
\hline \hline Variável & Descrição & Categorias \\
\hline IDADFEM & Idade em anos do paciente do gênero feminino (anos) \\
IDADMAS & Idade em anos do paciente do gênero masculino (anos) \\
COLS & Taxa de colesterol sem medicamento $(\mathrm{mg} / \mathrm{dl})$ \\
HDLM & Taxa de HDL com medicamento $(\mathrm{mg} / \mathrm{dl})$ \\
HDLS & Taxa de HDL sem medicamento $(\mathrm{mg} / \mathrm{dl})$ \\
LDLM & Taxa de LDL com medicamento $(\mathrm{mg} / \mathrm{dl})$ \\
LDLS & Taxa de LDL sem medicamento $(\mathrm{mg} / \mathrm{dl})$ \\
VLDLM & Taxa de VLDL com medicamento $(\mathrm{mg} / \mathrm{dl})$ \\
VLDLS & Taxa de VLDL sem medicamento $(\mathrm{mg} / \mathrm{dl})$ \\
TRIGM & Taxa de triglicérides com medicamento $(\mathrm{TG})$ \\
TRIGS & Taxa de triglicérides sem medicamento $(\mathrm{TG})$ \\
GLICM & Taxa de glicose com medicamento \\
GLICS & Taxa de glicose sem medicamento \\
C/H-M & Taxa de colesterol/HDL com medicamento $(\mathrm{mg} / \mathrm{dl})$ \\
C/H-S & Taxa de colesterol/HDL sem medicamento $(\mathrm{mg} / \mathrm{dl})$ \\
L/H-M & Taxa de LDL/HDL com medicamento $(\mathrm{mg} / \mathrm{dl})$ \\
L/H-S & Taxa de LDL/HDL sem medicamento $(\mathrm{mg} / \mathrm{dl})$ \\
\hline \hline
\end{tabular}

Tabela D.2: Continuação da descrição das variáveis da base de aplicação.

\begin{tabular}{lcc}
\hline \hline HA & Quantidade & $\%$ \\
\hline Não & 599 & $39,9 \%$ \\
Sim & 849 & $56,6 \%$ \\
Sem informação & 52 & $3,5 \%$ \\
\hline Total & 1500 & $100,0 \%$ \\
\hline \hline
\end{tabular}

Tabela D.3: Análise exploratória de $H A$.

\begin{tabular}{lcc}
\hline \hline DIAB & Quantidade & $\%$ \\
\hline Não & 1135 & $75,7 \%$ \\
Sim & 254 & $16,9 \%$ \\
Sem informação & 111 & $7,4 \%$ \\
\hline Total & 1500 & $100,0 \%$ \\
\hline \hline
\end{tabular}

Tabela D.4: Análise exploratória de DIAB. 


\begin{tabular}{lcc}
\hline \hline TABAG & Quantidade & $\%$ \\
\hline Não & 538 & $35,9 \%$ \\
Sim & 832 & $55,5 \%$ \\
Sem informação & 130 & $8,7 \%$ \\
\hline Total & 1500 & $100,0 \%$ \\
\hline \hline
\end{tabular}

Tabela D.5: Análise exploratória de TABAG.

\begin{tabular}{lcc}
\hline \hline ANGEST & Quantidade & $\%$ \\
\hline Não & 898 & $60,0 \%$ \\
Sim & 602 & $40,0 \%$ \\
\hline Total & 1500 & $100,0 \%$ \\
\hline \hline
\end{tabular}

Tabela D.6: Análise exploratória de ANGEST.

\begin{tabular}{lcc}
\hline \hline ANGINS & Quantidade & $\%$ \\
\hline Não & 998 & $66,5 \%$ \\
Sim & 502 & $33,5 \%$ \\
\hline Total & 1500 & $100,0 \%$ \\
\hline \hline
\end{tabular}

Tabela D.7: Análise exploratória de ANGINS.

\begin{tabular}{lcc}
\hline \hline ICC & Quantidade & $\%$ \\
\hline Não & 1297 & $86,5 \%$ \\
Sim & 203 & $13,5 \%$ \\
\hline Total & 1500 & $100,0 \%$ \\
\hline \hline
\end{tabular}

Tabela D.8: Análise exploratória de ICC.

\begin{tabular}{lcc}
\hline \hline ARRIT & Quantidade & $\%$ \\
\hline Não & 1333 & $88,9 \%$ \\
Sim & 167 & $11,1 \%$ \\
\hline Total & 1500 & $100,0 \%$ \\
\hline \hline
\end{tabular}

Tabela D.9: Análise exploratória de ARRIT. 


\begin{tabular}{lcc}
\hline \hline ARTER & Quantidade & $\%$ \\
\hline Nenhuma & 1312 & $87,5 \%$ \\
Carótida & 94 & $6,3 \%$ \\
Aorta & 67 & $4,5 \%$ \\
Carótida+Aorta & 8 & $0,5 \%$ \\
Sem informação & 19 & $1,3 \%$ \\
\hline Total & 1500 & $100,0 \%$ \\
\hline \hline
\end{tabular}

Tabela D.10: Análise exploratória de ARTER.

\begin{tabular}{lcc}
\hline \hline CD & Quantidade & $\%$ \\
\hline 0 & 812 & $54,1 \%$ \\
1 & 89 & $5,9 \%$ \\
2 & 128 & $8,5 \%$ \\
3 & 229 & $15,3 \%$ \\
4 & 242 & $16,1 \%$ \\
\hline Total & 1500 & $100,0 \%$ \\
\hline
\end{tabular}

Tabela D.11: Análise exploratória de CD.

\begin{tabular}{lcc}
\hline \hline DA & Quantidade & $\%$ \\
\hline 0 & 682 & $45,5 \%$ \\
1 & 108 & $7,2 \%$ \\
2 & 129 & $8,6 \%$ \\
3 & 351 & $23,4 \%$ \\
4 & 230 & $15,3 \%$ \\
\hline Total & 1500 & $100,0 \%$ \\
\hline \hline
\end{tabular}

Tabela D.12: Análise exploratória de DA. 


\begin{tabular}{lcc}
\hline \hline CX & Quantidade & $\%$ \\
\hline 0 & 955 & $63,7 \%$ \\
1 & 82 & $5,5 \%$ \\
2 & 120 & $8,0 \%$ \\
3 & 223 & $14,9 \%$ \\
4 & 120 & $8,0 \%$ \\
\hline Total & 1500 & $100,0 \%$ \\
\hline \hline
\end{tabular}

Tabela D.13: Análise exploratória de $C X$.

\begin{tabular}{lcc}
\hline \hline GENERO & Quantidade & $\%$ \\
\hline Feminino & 421 & $28,1 \%$ \\
Masculino & 1079 & $71,9 \%$ \\
\hline Total & 1500 & $100,0 \%$ \\
\hline \hline
\end{tabular}

Tabela D.14: Análise exploratória de GENERO.

\begin{tabular}{lcc}
\hline \hline OBESO & Quantidade & $\%$ \\
\hline Não & 1278 & $85,2 \%$ \\
Sim & 144 & $9,6 \%$ \\
Sem informação & 78 & $5,2 \%$ \\
\hline Total & 1500 & $100,0 \%$ \\
\hline \hline
\end{tabular}

Tabela D.15: Análise exploratória de OBESO.

\begin{tabular}{lcc}
\hline \hline AH2 & Quantidade & $\%$ \\
\hline Não & 630 & $42,0 \%$ \\
Sim & 870 & $58,0 \%$ \\
\hline Total & 1500 & $100,0 \%$ \\
\hline \hline
\end{tabular}

Tabela D.16: Análise exploratória de AH2.

\begin{tabular}{lcc}
\hline \hline INFARTO & Quantidade & $\%$ \\
\hline Não & 1023 & $68,2 \%$ \\
Sim & 477 & $31,8 \%$ \\
\hline Total & 1500 & $100,0 \%$ \\
\hline \hline
\end{tabular}

Tabela D.17: Análise exploratória de INFARTO. 


\begin{tabular}{lcccccccc}
\hline \hline Covariável & $\mathrm{N}$ & Sem inf. & Média & Mín. & $1^{\circ}$ Quartil & $2^{\circ}$ Quartil & $3^{\circ}$ Quartil & Máx. \\
\hline PLAQ & 808 & 692 & 280,8 & 94 & 232,0 & 264,0 & 319,5 & 798,0 \\
FIB & 553 & 947 & 346,6 & 127,0 & 263,0 & 326,0 & 408,0 & 894,0 \\
C/H & 996 & 504 & 5,6 & 1,0 & 4,0 & 5,0 & 7,0 & 18,0 \\
L/H & 900 & 600 & 3,6 & 1,0 & 3,0 & 3,0 & 4,0 & 19,0 \\
COLM & 1247 & 253 & 27,2 & 0 & 0 & 0 & 0 & 360,0 \\
COLS & 1247 & 253 & 190,5 & 0 & 169,0 & 205,0 & 238,0 & 620,0 \\
HDLM & 1000 & 500 & 5,4 & 0 & 0 & 0 & 0 & 70,0 \\
HDLS & 1000 & 500 & 36,1 & 0 & 28,0 & 37,0 & 47,0 & 196,0 \\
LDLM & 905 & 595 & 19,4 & 0 & 0 & 0 & 0 & 261,0 \\
LDLS & 905 & 595 & 120,5 & 0 & 92,5 & 128,0 & 159,0 & 544,0 \\
VLDLM & 805 & 695 & 5,0 & 0 & 0 & 0 & 0 & 124,0 \\
VLDLS & 805 & 695 & 28,7 & 0 & 17,0 & 27,0 & 39,0 & 191,0 \\
IDADFEM & 1500 & 0 & 16,8 & 0 & 0 & 0 & 45,3 & 87,0 \\
IDADMAS & 1500 & 0 & 41,2 & 0 & 0 & 52,0 & 62,0 & 87,0 \\
TRIGM & 1209 & 291 & 23,1 & 0 & 0 & 0 & 0 & 728,0 \\
TRIGS & 1209 & 291 & 152,5 & 0 & 92,0 & 137,0 & 201,0 & 999,0 \\
GLICM & 1194 & 306 & 32,3 & 0 & 0 & 0 & 0 & 405,0 \\
GLICS & 1194 & 306 & 77,4 & 0 & 76,0 & 90,0 & 100,0 & 325,0 \\
C/H-M & 996 & 504 & 0,8 & 0 & 0 & 0 & 0 & 11,0 \\
C/H-S & 996 & 504 & 4,8 & 0 & 4,0 & 5,0 & 6,0 & 18,0 \\
L/H-M & 900 & 600 & 0,5 & 0 & 0 & 0 & 0 & 8,0 \\
L/H-S & 900 & 600 & 3,1 & 0 & 2,0 & 3,0 & 4,0 & 19,0 \\
\hline \hline
\end{tabular}

Tabela D.18: Análise exploratória de COLM, IDADFEM, IDADMAS, COLS, TRIGM, TRIGS, GLICM e GLICS. 


\begin{tabular}{lccccc}
\hline \hline & HA & DIAB & TABAG & ANGEST & ANGINS \\
\hline (Intercepto) & $0.40(0.08)^{* * *}$ & $0.46(0.06)^{* * *}$ & $0.31(0.09)^{* * *}$ & $0.78(0.07)^{* * *}$ & $0.32(0.06)^{* * *}$ \\
HA1 & $0.34(0.11)^{* * *}$ & & & & \\
DIAB1 & & $0.99(0.17)^{* * *}$ & & & \\
TABAG1 & & & $0.53(0.12)^{* * *}$ & & \\
ANGEST1 & & & $-0.45(0.11)^{* * *}$ & \\
ANGINS1 & & & & $0.91(0.12)^{* * *}$ \\
\hline AIC & 1878.48 & 1764.06 & 1753.81 & 1941.71 & 1900.92 \\
Num. obs. & 1448 & 1389 & 1370 & 1500 & 1500 \\
\hline \hline
\end{tabular}

${ }^{* * *} p<0.01,{ }^{* *} p<0.05,{ }^{*} p<0.1$

Tabela D.19: Estimativas dos coeficientes e respectivos erros padrão entre parênteses dos modelos de regressão logística univariados de HA, DIAB, TABAG, ANGEST e ANGINS.

\begin{tabular}{|c|c|c|c|c|c|}
\hline & $\mathrm{ICC}$ & ARRIT & ARTER & $\mathrm{CD}$ & $\mathrm{DA}$ \\
\hline (Intercepto) & $0.62(0.06)^{* * *}$ & $0.66(0.06)^{* * *}$ & $0.51(0.06)^{* * *}$ & $-0.52(0.07)^{* * *}$ & $-0.92(0.08)^{* * *}$ \\
\hline ICC1 & $-0.23(0.15)$ & & & & \\
\hline ARRIT1 & & $-0.63(0.17)^{* * *}$ & & & \\
\hline ARTER1 & & & $0.62(0.25)^{* *}$ & & \\
\hline ARTER2 & & & $0.92(0.31)^{* * *}$ & & \\
\hline ARTER3 & & & $0.59(0.82)$ & & \\
\hline CD1 & & & & $1.46(0.25)^{* * *}$ & \\
\hline CD2 & & & & $20.09(950.53)$ & \\
\hline CD3 & & & & $20.09(710.65)$ & \\
\hline $\mathrm{CD} 4$ & & & & $20.09(691.29)$ & \\
\hline DA1 & & & & & $1.18(0.21)^{* * *}$ \\
\hline $\mathrm{DA} 2$ & & & & & $20.49(946.84)$ \\
\hline DA3 & & & & & $20.49(574.01)$ \\
\hline DA4 & & & & & $20.49(709.10)$ \\
\hline $\mathrm{AIC}$ & 1956.18 & 1944.17 & 1924.49 & 1187.49 & 972.36 \\
\hline Num. obs. & 1500 & 1500 & 1481 & 1500 & 1500 \\
\hline
\end{tabular}

$$
{ }^{* * *} p<0.01,{ }^{* *} p<0.05,{ }^{*} p<0.1
$$

Tabela D.20: Estimativas dos coeficientes e respectivos erros padrão entre parênteses dos modelos de regressão logística univariados de ICC, ARRIT, ARTER, CD e DA. 


\begin{tabular}{|c|c|c|c|c|c|}
\hline & CX & GENERO & OBESO & $\mathrm{AH} 2$ & INFARTO \\
\hline (Intercepto) & $-0.18(0.06)^{* * *}$ & $-0.18(0.10)^{*}$ & $0.63(0.06)^{* * *}$ & $0.47(0.08)^{* * *}$ & $0.06(0.06)$ \\
\hline CX1 & $1.76(0.30)^{* * *}$ & & & & \\
\hline CX2 & $18.75(595.43)$ & & & & \\
\hline CX3 & $18.75(436.79)$ & & & & \\
\hline CX4 & $18.75(595.43)$ & & & & \\
\hline GENERO1 & & $1.10(0.12)^{* * *}$ & & & \\
\hline OBESO1 & & & $-0.49(0.18)^{* * *}$ & & \\
\hline AH21 & & & & $0.21(0.11)^{*}$ & \\
\hline INFARTO1 & & & & & $2.33(0.18)^{* * *}$ \\
\hline $\mathrm{AIC}$ & 1400.93 & 1871.23 & 1854.94 & 1954.87 & 1695.95 \\
\hline Num. obs. & 1500 & 1500 & 1422 & 1500 & 1500 \\
\hline
\end{tabular}

${ }^{* * *} p<0.01,{ }^{* *} p<0.05,{ }^{*} p<0.1$

Tabela D.21: Estimativas dos coeficientes e respectivos erros padrão entre parênteses dos modelos de regressão logística univariados de CX, GENERO, OBESO, AHQ e INFARTO.

\begin{tabular}{lcccc}
\hline \hline & COLM & IDADFEM & IDADMAS & COLS \\
\hline (Intercepto) & $0.74(0.06)^{* * *}$ & $0.61(0.06)^{* * *}$ & $0.63(0.06)^{* * *}$ & $0.73(0.06)^{* * *}$ \\
COLM & $0.24(0.07)^{* * *}$ & & & \\
IDADFEM & & $-0.44(0.05)^{* * *}$ & & \\
IDADMAS & & & $0.62(0.06)^{* * *}$ & \\
COLS & & & & $-0.14(0.06)^{* *}$ \\
\hline AIC & 1566.57 & 1891.00 & 1828.33 & 1574.13 \\
Num. obs. & 1247 & 1500 & 1500 & 1247 \\
\hline \hline & & & & \\
${ }^{* * *} p<0.01,{ }^{* *} p<0.05,{ }^{*} p<0.1$ & & &
\end{tabular}

Tabela D.22: Estimativas dos coeficientes e respectivos erros padrão entre parênteses dos modelos de regressão logistica univariados de COLM, IDADFEM, IDADMAS e COLS. 


\begin{tabular}{lcccc}
\hline \hline & TRIGM & TRIGS & GLICM & GLICS \\
\hline (Intercepto) & $0.76(0.06)^{* * *}$ & $0.75(0.06)^{* * *}$ & $0.76(0.06)^{* * *}$ & $0.73(0.06)^{* * *}$ \\
TRIGM & $0.30(0.08)^{* * *}$ & & & \\
TRIGS & & $-0.02(0.06)$ & & \\
GLICM & & & $0.43(0.08)^{* * *}$ & \\
GLICS & & & & $-0.21(0.06)^{* * *}$ \\
\hline AIC & 1506.37 & 1522.89 & 1478.76 & 1502.94 \\
Num. obs. & 1209 & 1209 & 1194 & 1194 \\
\hline \hline
\end{tabular}

${ }^{* * *} p<0.01,{ }^{* *} p<0.05,{ }^{*} p<0.1$

Tabela D.23: Estimativas dos coeficientes e respectivos erros padrão entre parênteses dos modelos de regressão logística univariados de TRIGM, TRIGS, GLICM e GLICS.

\begin{tabular}{lcccc}
\hline \hline & DIAB & TABAG & ANGEST & \multicolumn{1}{c}{ ANGINS } \\
\hline (Intercepto) & $0.32(0.05)^{* * *}$ & $0.55(0.15)^{* * *}$ & $0.33(0.15)^{* *}$ & $0.47(0.15)^{* * *}$ \\
DIAB1 & & $-0.08(0.09)$ & $-0.13(0.09)$ & $-0.27(0.09)^{* * *}$ \\
TABAG1 & $-0.01(0.01)$ & & $-0.03(0.03)$ & $0.02(0.03)$ \\
ANGEST1 & $-0.02(0.01)$ & $-0.03(0.03)$ & & $-0.33(0.03)^{* * *}$ \\
ANGINS1 & $-0.04(0.01)^{* * *}$ & $0.02(0.03)$ & $-0.32(0.03)^{* * *}$ & \\
ARRIT1 & $-0.03(0.02)^{*}$ & $-0.09(0.05)^{*}$ & $-0.15(0.05)^{* * *}$ & $-0.10(0.05)^{* *}$ \\
GENERO1 & $-0.14(0.07)^{* *}$ & $0.09(0.20)$ & $0.42(0.20)^{* *}$ & $0.11(0.20)$ \\
INFARTO1 & $0.01(0.01)$ & $0.09(0.03)^{* * *}$ & $-0.25(0.03)^{* * *}$ & $-0.09(0.03)^{* * *}$ \\
IDADFEM & $-0.05(0.03)^{*}$ & $-0.21(0.08)^{* * *}$ & $0.16(0.08)^{* *}$ & $0.05(0.08)$ \\
IDADMAS & $0.02(0.01)^{*}$ & $-0.09(0.04)^{* *}$ & $-0.04(0.04)$ & $0.02(0.04)$ \\
COLM & $-0.01(0.01)$ & $0.00(0.03)$ & $-0.02(0.03)$ & $-0.02(0.03)$ \\
TRIGM & $0.02(0.01)^{*}$ & $-0.01(0.03)$ & $0.02(0.03)$ & $0.04(0.03)$ \\
GLICM & $0.35(0.01)^{* * *}$ & $0.01(0.03)$ & $0.03(0.03)$ & $0.12(0.03)^{* * *}$ \\
\hline R $^{2}$ & 0.83 & 0.15 & 0.18 & 0.12 \\
Adj. R ${ }^{2}$ & 0.83 & 0.14 & 0.17 & 0.11 \\
Num. obs. & 1025 & 1025 & 1025 & 1025 \\
\hline \hline
\end{tabular}

${ }^{* * *} p<0.01,{ }^{* *} p<0.05,{ }^{*} p<0.1$

Tabela D.24: Modelos de regressão linear com variáveis resposta dadas por DIAB, TABAG, ANGEST e ANGINS. 


\begin{tabular}{|c|c|c|c|c|}
\hline & ARRIT & GENERO & INFARTO & IDADFEM \\
\hline (Intercepto) & $0.28(0.10)^{* * *}$ & $0.75(0.01)^{* * *}$ & $0.21(0.15)$ & $1.71(0.03)^{* * *}$ \\
\hline DIAB1 & $-0.11(0.06)^{*}$ & $-0.03(0.01)^{* *}$ & $0.11(0.09)$ & $-0.06(0.04)^{*}$ \\
\hline TABAG1 & $-0.04(0.02)^{*}$ & $0.00(0.00)$ & $0.09(0.03)^{* * *}$ & $-0.04(0.01)^{* * *}$ \\
\hline ANGEST1 & $-0.07(0.02)^{* * *}$ & $0.01(0.01)^{* *}$ & $-0.25(0.03)^{* * *}$ & $0.03(0.01)^{* *}$ \\
\hline ANGINS1 & $-0.04(0.02)^{* *}$ & $0.00(0.01)$ & $-0.09(0.03)^{* * *}$ & $0.01(0.01)$ \\
\hline ARRIT1 & & $-0.01(0.01)$ & $-0.06(0.05)$ & $0.01(0.02)$ \\
\hline GENERO1 & $-0.12(0.13)$ & & $0.26(0.20)$ & $-2.25(0.04)^{* * *}$ \\
\hline INFARTO1 & $-0.03(0.02)$ & $0.01(0.00)$ & & $0.01(0.01)$ \\
\hline IDADFEM & $0.02(0.05)$ & $-0.34(0.01)^{* * *}$ & $0.08(0.08)$ & \\
\hline IDADMAS & $0.07(0.03)^{* * *}$ & $0.09(0.01)^{* * *}$ & $0.00(0.04)$ & $0.00(0.02)$ \\
\hline COLM & $0.00(0.02)$ & $0.01(0.00)$ & $0.01(0.03)$ & $0.03(0.01)^{* *}$ \\
\hline TRIGM & $0.01(0.02)$ & $-0.01(0.00)^{* *}$ & $0.00(0.03)$ & $-0.04(0.01)^{* * *}$ \\
\hline GLICM & $0.03(0.02)$ & $0.01(0.01)^{*}$ & $0.00(0.03)$ & $0.03(0.01)^{* *}$ \\
\hline $\mathrm{R}^{2}$ & 0.03 & 0.97 & 0.10 & 0.97 \\
\hline Adj. $R^{2}$ & 0.02 & 0.97 & 0.09 & 0.97 \\
\hline Num. obs. & 1025 & 1025 & 1025 & 1025 \\
\hline
\end{tabular}

${ }^{* * *} p<0.01,{ }^{* *} p<0.05,{ }^{*} p<0.1$

Tabela D.25: Modelos de regressão linear com variáveis resposta dadas por ARRIT, GENERO, INFARTO e IDADFEM. 


\begin{tabular}{lcccc}
\hline \hline & IDADMAS & COLM & TRIGM & GLICM \\
\hline Intercepto $)$ & $-1.62(0.10)^{* * *}$ & $-0.19(0.16)$ & $0.28(0.15)^{*}$ & $-0.72(0.14)^{* * *}$ \\
DIAB1 & $0.11(0.07)^{*}$ & $-0.09(0.09)$ & $0.17(0.09)^{*}$ & $2.33(0.03)^{* * *}$ \\
TABAG1 & $-0.05(0.02)^{* *}$ & $0.00(0.03)$ & $-0.01(0.03)$ & $0.01(0.03)$ \\
ANGEST1 & $-0.03(0.02)$ & $-0.02(0.03)$ & $0.02(0.03)$ & $0.03(0.03)$ \\
ANGINS1 & $0.01(0.02)$ & $-0.02(0.03)$ & $0.04(0.03)$ & $0.10(0.03)^{* * *}$ \\
ARRIT1 & $0.10(0.04)^{* * *}$ & $-0.01(0.05)$ & $0.02(0.05)$ & $0.05(0.04)$ \\
GENERO1 & $2.16(0.13)^{* * *}$ & $0.30(0.20)$ & $-0.44(0.20)^{* *}$ & $0.31(0.18)^{*}$ \\
INFARTO1 & $0.00(0.02)$ & $0.01(0.03)$ & $0.00(0.03)$ & $0.00(0.03)$ \\
IDADFEM & $-0.01(0.06)$ & $0.16(0.08)^{* *}$ & $-0.22(0.08)^{* * *}$ & $0.15(0.07)^{* *}$ \\
IDADMAS & & $0.04(0.04)$ & $-0.03(0.04)$ & $-0.01(0.04)$ \\
COLM & $0.02(0.02)$ & & $0.88(0.01)^{* * *}$ & $0.02(0.03)$ \\
TRIGM & $-0.02(0.02)$ & $0.89(0.01)^{* * *}$ & & $-0.03(0.03)$ \\
GLICM & $-0.01(0.03)$ & $0.02(0.03)$ & $-0.04(0.03)$ & \\
\hline R $^{2}$ & 0.88 & 0.79 & 0.79 & 0.83 \\
Adj. R & 0.88 & 0.79 & 0.79 & 0.83 \\
Num. obs. & 1025 & 1025 & 1025 & 1025 \\
\hline \hline
\end{tabular}

${ }^{* * *} p<0.01,{ }^{* *} p<0.05,{ }^{*} p<0.1$

Tabela D.26: Modelos de regressão linear com variáveis resposta dadas por IDADMAS, COLM, TRIGM e GLICM. 


\begin{tabular}{|c|c|c|c|c|c|c|c|}
\hline & (Intercepto) & DIAB & TABAG & ANGEST & ANGINS & ARRIT & GENERO \\
\hline (Intercepto) & 0,7487 & $-0,1084$ & $-0,0159$ & $-0,0118$ & $-0,0190$ & $-0,0196$ & $-0,9225$ \\
\hline DIAB & $-0,1084$ & 0,4019 & 0,0007 & 0,0005 & 0,0074 & 0,0127 & 0,0317 \\
\hline TABAG & $-0,0159$ & 0,0007 & 0,0326 & $-0,0004$ & $-0,0015$ & 0,0035 & $-0,0041$ \\
\hline ANGEST & $-0,0118$ & 0,0005 & $-0,0004$ & 0,0347 & 0,0156 & 0,0054 & $-0,0137$ \\
\hline ANGINS & $-0,0190$ & 0,0074 & $-0,0015$ & 0,0156 & 0,0419 & 0,0031 & $-0,0021$ \\
\hline ARRIT & $-0,0196$ & 0,0127 & 0,0035 & 0,0054 & 0,0031 & 0,0773 & 0,0041 \\
\hline GENERO & $-0,9225$ & 0,0317 & $-0,0041$ & $-0,0137$ & $-0,0021$ & 0,0041 & 1,2270 \\
\hline INFARTO & $-0,0151$ & $-0,0104$ & $-0,0053$ & 0,0139 & 0,0122 & 0,0001 & 0,0033 \\
\hline COLM & 0,0186 & 0,0047 & 0,0016 & $-0,0010$ & 0,0007 & 0,0018 & $-0,0298$ \\
\hline IDADFEM & $-0,3069$ & 0,0104 & 0,0047 & $-0,0034$ & 0,0039 & $-0,0060$ & 0,4033 \\
\hline IDADMAS & 0,0973 & $-0,0058$ & 0,0008 & 0,0045 & 0,0064 & $-0,0089$ & $-0,1297$ \\
\hline TRIGM & $-0,0301$ & $-0,0136$ & $-0,0024$ & 0,0006 & $-0,0004$ & $-0,0052$ & 0,0519 \\
\hline \multirow[t]{2}{*}{ GLICM } & 0,0427 & $-0,1656$ & 0,0005 & 0,0014 & $-0,0024$ & $-0,0044$ & $-0,0104$ \\
\hline & INFARTO & COLM & $\overline{\text { IDADFEM }}$ & IDADMAS & TRIGM & GLICM & \\
\hline (Intercepto) & $-0,0151$ & 0,0186 & $-0,3069$ & 0,0973 & $-0,0301$ & 0,0427 & \\
\hline DIAB & $-0,0104$ & 0,0047 & 0,0104 & $-0,0058$ & $-0,0136$ & $-0,1656$ & \\
\hline TABAG & $-0,0053$ & 0,0016 & 0,0047 & 0,0008 & $-0,0024$ & 0,0005 & \\
\hline ANGEST & 0,0139 & $-0,0010$ & $-0,0034$ & 0,0045 & 0,0006 & 0,0014 & \\
\hline ANGINS & 0,0122 & 0,0007 & 0,0039 & 0,0064 & $-0,0004$ & $-0,0024$ & \\
\hline ARRIT & 0,0001 & 0,0018 & $-0,0060$ & $-0,0089$ & $-0,0052$ & $-0,0044$ & \\
\hline GENERO & 0,0033 & $-0,0298$ & 0,4033 & $-0,1297$ & 0,0519 & $-0,0104$ & \\
\hline INFARTO & 0,0607 & 0,0001 & 0,0076 & 0,0088 & 0,0019 & 0,0041 & \\
\hline COLM & 0,0001 & 0,0487 & $-0,0130$ & $-0,0005$ & $-0,0549$ & $-0,0006$ & \\
\hline IDADFEM & 0,0076 & $-0,0130$ & 0,1792 & 0,0021 & 0,0223 & $-0,0048$ & \\
\hline IDADMAS & 0,0088 & $-0,0005$ & 0,0021 & 0,0640 & 0,0011 & 0,0009 & \\
\hline TRIGM & 0,0019 & $-0,0549$ & 0,0223 & 0,0011 & 0,0785 & 0,0035 & \\
\hline GLICM & 0,0041 & $-0,0006$ & $-0,0048$ & 0,0009 & 0,0035 & 0,0796 & \\
\hline
\end{tabular}

Tabela D.27: Matriz de covariância estimada dos estimadores dos parâmetros do Modelo de Regressão Logística através de Máxima Verossimilhança. 


\begin{tabular}{|c|c|c|c|c|c|c|c|}
\hline & (Intercepto) & DIAB & TABAG & ANGEST & ANGINS & ARRIT & GENERO \\
\hline (Intercepto) & 0,5139 & $-0,0825$ & $-0,0143$ & $-0,0124$ & $-0,0166$ & $-0,0164$ & $-0,6216$ \\
\hline DIAB & $-0,0825$ & 0,3639 & 0,0004 & 0,0004 & 0,0064 & 0,0110 & 0,0086 \\
\hline TABAG & $-0,0143$ & 0,0004 & 0,0323 & $-0,0004$ & $-0,0016$ & 0,0034 & $-0,0058$ \\
\hline ANGEST & $-0,0124$ & 0,0004 & $-0,0004$ & 0,0343 & 0,0153 & 0,0053 & $-0,0124$ \\
\hline ANGINS & $-0,0166$ & 0,0064 & $-0,0016$ & 0,0153 & 0,0414 & 0,0029 & $-0,0044$ \\
\hline ARRIT & $-0,0164$ & 0,0110 & 0,0034 & 0,0053 & 0,0029 & 0,0759 & 0,0007 \\
\hline GENERO & $-0,6216$ & 0,0086 & $-0,0058$ & $-0,0124$ & $-0,0044$ & 0,0007 & 0,8375 \\
\hline INFARTO & $-0,0127$ & $-0,0096$ & $-0,0053$ & 0,0136 & 0,0118 & 0,0001 & 0,0003 \\
\hline COLM & 0,0114 & 0,0046 & 0,0016 & $-0,0010$ & 0,0007 & 0,0017 & $-0,0201$ \\
\hline IDADFEM & $-0,2060$ & 0,0028 & 0,0041 & $-0,0030$ & 0,0030 & $-0,0069$ & 0,2728 \\
\hline IDADMAS & 0,0669 & $-0,0032$ & 0,0010 & 0,0042 & 0,0065 & $-0,0082$ & $-0,0903$ \\
\hline TRIGM & $-0,0181$ & $-0,0129$ & $-0,0024$ & 0,0007 & $-0,0005$ & $-0,0051$ & 0,0356 \\
\hline \multirow[t]{2}{*}{ GLICM } & 0,0325 & $-0,1496$ & 0,0006 & 0,0015 & $-0,0020$ & $-0,0037$ & $-0,0016$ \\
\hline & INFARTO & COLM & IDADFEM & IDADMAS & TRIGM & GLICM & \\
\hline (Intercepto) & $-0,0127$ & 0,0114 & $-0,2060$ & 0,0669 & $-0,0181$ & 0,0325 & \\
\hline DIAB & $-0,0096$ & 0,0046 & 0,0028 & $-0,0032$ & $-0,0129$ & $-0,1496$ & \\
\hline TABAG & $-0,0053$ & 0,0016 & 0,0041 & 0,0010 & $-0,0024$ & 0,0006 & \\
\hline ANGEST & 0,0136 & $-0,0010$ & $-0,0030$ & 0,0042 & 0,0007 & 0,0015 & \\
\hline ANGINS & 0,0118 & 0,0007 & 0,0030 & 0,0065 & $-0,0005$ & $-0,0020$ & \\
\hline ARRIT & 0,0001 & 0,0017 & $-0,0069$ & $-0,0082$ & $-0,0051$ & $-0,0037$ & \\
\hline GENERO & 0,0003 & $-0,0201$ & 0,2728 & $-0,0903$ & 0,0356 & $-0,0016$ & \\
\hline INFARTO & 0,0598 & 0,0002 & 0,0064 & 0,0088 & 0,0017 & 0,0038 & \\
\hline COLM & 0,0002 & 0,0474 & $-0,0096$ & $-0,0013$ & $-0,0532$ & $-0,0006$ & \\
\hline IDADFEM & 0,0064 & $-0,0096$ & 0,1345 & 0,0143 & 0,0167 & $-0,0018$ & \\
\hline IDADMAS & 0,0088 & $-0,0013$ & 0,0143 & 0,0591 & 0,0024 & $-0,0001$ & \\
\hline TRIGM & 0,0017 & $-0,0532$ & 0,0167 & 0,0024 & 0,0760 & 0,0032 & \\
\hline GLICM & 0,0038 & $-0,0006$ & $-0,0018$ & $-0,0001$ & 0,0032 & 0,0729 & \\
\hline
\end{tabular}

Tabela D.28: Matriz de covariância estimada dos estimadores dos parâmetros do Modelo de Regressão em Cristas com o primeiro parâmetro em cristas. 


\begin{tabular}{|c|c|c|c|c|c|c|c|}
\hline & (Intercepto) & DIAB & TABAG & ANGEST & ANGINS & ARRIT & GENERO \\
\hline (Intercepto) & 0,1124 & $-0,0280$ & $-0,0110$ & $-0,0122$ & $-0,0113$ & $-0,0094$ & $-0,1120$ \\
\hline DIAB & $-0,0280$ & 0,2034 & $-0,0007$ & $-0,0005$ & 0,0026 & 0,0046 & $-0,0165$ \\
\hline TABAG & $-0,0110$ & $-0,0007$ & 0,0304 & $-0,0006$ & $-0,0017$ & 0,0027 & $-0,0080$ \\
\hline ANGEST & $-0,0122$ & $-0,0005$ & $-0,0006$ & 0,0316 & 0,0131 & 0,0042 & $-0,0092$ \\
\hline ANGINS & $-0,0113$ & 0,0026 & $-0,0017$ & 0,0131 & 0,0381 & 0,0021 & $-0,0072$ \\
\hline ARRIT & $-0,0094$ & 0,0046 & 0,0027 & 0,0042 & 0,0021 & 0,0675 & $-0,0038$ \\
\hline GENERO & $-0,1120$ & $-0,0165$ & $-0,0080$ & $-0,0092$ & $-0,0072$ & $-0,0038$ & 0,1723 \\
\hline INFARTO & $-0,0078$ & $-0,0057$ & $-0,0048$ & 0,0114 & 0,0097 & 0,0000 & $-0,0043$ \\
\hline COLM & 0,0000 & 0,0022 & 0,0013 & $-0,0010$ & 0,0006 & 0,0011 & $-0,0034$ \\
\hline IDADFEM & $-0,0354$ & $-0,0053$ & 0,0029 & $-0,0023$ & 0,0012 & $-0,0068$ & 0,0505 \\
\hline IDADMAS & 0,0149 & 0,0008 & 0,0011 & 0,0034 & 0,0057 & $-0,0060$ & $-0,0225$ \\
\hline TRIGM & 0,0010 & $-0,0068$ & $-0,0019$ & 0,0007 & $-0,0004$ & $-0,0038$ & 0,0074 \\
\hline \multirow[t]{2}{*}{ GLICM } & 0,0110 & $-0,0821$ & 0,0010 & 0,0017 & $-0,0004$ & $-0,0012$ & 0,0073 \\
\hline & INFARTO & COLM & $\overline{\text { IDADFEM }}$ & IDADMAS & TRIGM & GLICM & \\
\hline (Intercepto) & $-0,0078$ & 0,0000 & $-0,0354$ & 0,0149 & 0,0010 & 0,0110 & \\
\hline DIAB & $-0,0057$ & 0,0022 & $-0,0053$ & 0,0008 & $-0,0068$ & $-0,0821$ & \\
\hline TABAG & $-0,0048$ & 0,0013 & 0,0029 & 0,0011 & $-0,0019$ & 0,0010 & \\
\hline ANGEST & 0,0114 & $-0,0010$ & $-0,0023$ & 0,0034 & 0,0007 & 0,0017 & \\
\hline ANGINS & 0,0097 & 0,0006 & 0,0012 & 0,0057 & $-0,0004$ & $-0,0004$ & \\
\hline ARRIT & 0,0000 & 0,0011 & $-0,0068$ & $-0,0060$ & $-0,0038$ & $-0,0012$ & \\
\hline GENERO & $-0,0043$ & $-0,0034$ & 0,0505 & $-0,0225$ & 0,0074 & 0,0073 & \\
\hline INFARTO & 0,0540 & 0,0004 & 0,0035 & 0,0075 & 0,0010 & 0,0022 & \\
\hline COLM & 0,0004 & 0,0405 & $-0,0032$ & $-0,0020$ & $-0,0440$ & 0,0002 & \\
\hline IDADFEM & 0,0035 & $-0,0032$ & 0,0548 & 0,0313 & 0,0059 & 0,0012 & \\
\hline IDADMAS & 0,0075 & $-0,0020$ & 0,0313 & 0,0461 & 0,0037 & $-0,0014$ & \\
\hline TRIGM & 0,0010 & $-0,0440$ & 0,0059 & 0,0037 & 0,0636 & 0,0010 & \\
\hline GLICM & 0,0022 & 0,0002 & 0,0012 & $-0,0014$ & 0,0010 & 0,0442 & \\
\hline
\end{tabular}

Tabela D.29: Matriz de covariância estimada dos estimadores dos parâmetros do Modelo de Regressão em Cristas com o segundo parâmetro em cristas. 


\begin{tabular}{|c|c|c|c|c|c|c|c|}
\hline & (Intercepto) & DIAB & TABAG & ANGEST & ANGINS & ARRIT & GENERO \\
\hline (Intercepto) & 0,1738 & $-0,0385$ & $-0,0117$ & $-0,0126$ & $-0,0125$ & $-0,0108$ & $-0,1886$ \\
\hline DIAB & $-0,0385$ & 0,2494 & $-0,0004$ & $-0,0002$ & 0,0036 & 0,0064 & $-0,0159$ \\
\hline TABAG & $-0,0117$ & $-0,0004$ & 0,0311 & $-0,0005$ & $-0,0016$ & 0,0029 & $-0,0079$ \\
\hline ANGEST & $-0,0126$ & $-0,0002$ & $-0,0005$ & 0,0326 & 0,0139 & 0,0046 & $-0,0100$ \\
\hline ANGINS & $-0,0125$ & 0,0036 & $-0,0016$ & 0,0139 & 0,0393 & 0,0024 & $-0,0071$ \\
\hline ARRIT & $-0,0108$ & 0,0064 & 0,0029 & 0,0046 & 0,0024 & 0,0705 & $-0,0034$ \\
\hline GENERO & $-0,1886$ & $-0,0159$ & $-0,0079$ & $-0,0100$ & $-0,0071$ & $-0,0034$ & 0,2737 \\
\hline INFARTO & $-0,0088$ & $-0,0069$ & $-0,0050$ & 0,0122 & 0,0105 & 0,0000 & $-0,0038$ \\
\hline COLM & 0,0015 & 0,0030 & 0,0014 & $-0,0010$ & 0,0007 & 0,0013 & $-0,0060$ \\
\hline IDADFEM & $-0,0610$ & $-0,0052$ & 0,0031 & $-0,0025$ & 0,0015 & $-0,0072$ & 0,0842 \\
\hline IDADMAS & 0,0229 & 0,0003 & 0,0011 & 0,0037 & 0,0061 & $-0,0066$ & $-0,0330$ \\
\hline TRIGM & $-0,0016$ & $-0,0088$ & $-0,0021$ & 0,0007 & $-0,0004$ & $-0,0043$ & 0,0118 \\
\hline \multirow[t]{2}{*}{ GLICM } & 0,0152 & $-0,1014$ & 0,0009 & 0,0017 & $-0,0008$ & $-0,0019$ & 0,0073 \\
\hline & INFARTO & COLM & IDADFEM & IDADMAS & TRIGM & GLICM & \\
\hline (Intercepto) & $-0,0088$ & 0,0015 & $-0,0610$ & 0,0229 & $-0,0016$ & 0,0152 & \\
\hline DIAB & $-0,0069$ & 0,0030 & $-0,0052$ & 0,0003 & $-0,0088$ & $-0,1014$ & \\
\hline TABAG & $-0,0050$ & 0,0014 & 0,0031 & 0,0011 & $-0,0021$ & 0,0009 & \\
\hline ANGEST & 0,0122 & $-0,0010$ & $-0,0025$ & 0,0037 & 0,0007 & 0,0017 & \\
\hline ANGINS & 0,0105 & 0,0007 & 0,0015 & 0,0061 & $-0,0004$ & $-0,0008$ & \\
\hline ARRIT & 0,0000 & 0,0013 & $-0,0072$ & $-0,0066$ & $-0,0043$ & $-0,0019$ & \\
\hline GENERO & $-0,0038$ & $-0,0060$ & 0,0842 & $-0,0330$ & 0,0118 & 0,0073 & \\
\hline INFARTO & 0,0561 & 0,0004 & 0,0041 & 0,0081 & 0,0012 & 0,0027 & \\
\hline COLM & 0,0004 & 0,0429 & $-0,0043$ & $-0,0021$ & $-0,0471$ & $-0,0001$ & \\
\hline IDADFEM & 0,0041 & $-0,0043$ & 0,0680 & 0,0299 & 0,0078 & 0,0012 & \\
\hline IDADMAS & 0,0081 & $-0,0021$ & 0,0299 & 0,0494 & 0,0038 & $-0,0013$ & \\
\hline TRIGM & 0,0012 & $-0,0471$ & 0,0078 & 0,0038 & 0,0677 & 0,0017 & \\
\hline GLICM & 0,0027 & $-0,0001$ & 0,0012 & $-0,0013$ & 0,0017 & 0,0525 & \\
\hline
\end{tabular}

Tabela D.30: Matriz de covariância estimada dos estimadores dos dos parâmetros do Modelo de Regressão em Cristas com o terceiro parâmetro em cristas. 


\begin{tabular}{|c|c|c|c|c|c|c|c|}
\hline & (Intercepto) & DIAB & TABAG & ANGEST & ANGINS & ARRIT & GENERO \\
\hline (Intercepto) & 0,4175 & $-0,0712$ & $-0,0136$ & $-0,0126$ & $-0,0156$ & $-0,0150$ & $-0,4982$ \\
\hline DIAB & $-0,0712$ & 0,3424 & 0,0002 & 0,0003 & 0,0058 & 0,0101 & 0,0000 \\
\hline TABAG & $-0,0136$ & 0,0002 & 0,0321 & $-0,0004$ & $-0,0016$ & 0,0033 & $-0,0065$ \\
\hline ANGEST & $-0,0126$ & 0,0003 & $-0,0004$ & 0,0340 & 0,0151 & 0,0052 & $-0,0118$ \\
\hline ANGINS & $-0,0156$ & 0,0058 & $-0,0016$ & 0,0151 & 0,0411 & 0,0028 & $-0,0053$ \\
\hline ARRIT & $-0,0150$ & 0,0101 & 0,0033 & 0,0052 & 0,0028 & 0,0751 & $-0,0006$ \\
\hline GENERO & $-0,4982$ & 0,0000 & $-0,0065$ & $-0,0118$ & $-0,0053$ & $-0,0006$ & 0,6775 \\
\hline INFARTO & $-0,0116$ & $-0,0092$ & $-0,0052$ & 0,0134 & 0,0116 & 0,0001 & $-0,0009$ \\
\hline COLM & 0,0085 & 0,0044 & 0,0016 & $-0,0010$ & 0,0007 & 0,0016 & $-0,0162$ \\
\hline IDADFEM & $-0,1646$ & $-0,0001$ & 0,0038 & $-0,0029$ & 0,0026 & $-0,0072$ & 0,2192 \\
\hline IDADMAS & 0,0544 & $-0,0021$ & 0,0010 & 0,0041 & 0,0065 & $-0,0079$ & $-0,0741$ \\
\hline TRIGM & $-0,0132$ & $-0,0123$ & $-0,0023$ & 0,0007 & $-0,0005$ & $-0,0049$ & 0,0290 \\
\hline \multirow[t]{2}{*}{ GLICM } & 0,0281 & $-0,1406$ & 0,0007 & 0,0015 & $-0,0017$ & $-0,0033$ & 0,0016 \\
\hline & INFARTO & COLM & IDADFEM & IDADMAS & TRIGM & GLICM & \\
\hline (Intercepto) & $-0,0116$ & 0,0085 & $-0,1646$ & 0,0544 & $-0,0132$ & 0,0281 & \\
\hline DIAB & $-0,0092$ & 0,0044 & $-0,0001$ & $-0,0021$ & $-0,0123$ & $-0,1406$ & \\
\hline TABAG & $-0,0052$ & 0,0016 & 0,0038 & 0,0010 & $-0,0023$ & 0,0007 & \\
\hline ANGEST & 0,0134 & $-0,0010$ & $-0,0029$ & 0,0041 & 0,0007 & 0,0015 & \\
\hline ANGINS & 0,0116 & 0,0007 & 0,0026 & 0,0065 & $-0,0005$ & $-0,0017$ & \\
\hline ARRIT & 0,0001 & 0,0016 & $-0,0072$ & $-0,0079$ & $-0,0049$ & $-0,0033$ & \\
\hline GENERO & $-0,0009$ & $-0,0162$ & 0,2192 & $-0,0741$ & 0,0290 & 0,0016 & \\
\hline INFARTO & 0,0592 & 0,0002 & 0,0058 & 0,0087 & 0,0016 & 0,0036 & \\
\hline COLM & 0,0002 & 0,0467 & $-0,0082$ & $-0,0016$ & $-0,0521$ & $-0,0005$ & \\
\hline IDADFEM & 0,0058 & $-0,0082$ & 0,1160 & 0,0192 & 0,0143 & $-0,0007$ & \\
\hline IDADMAS & 0,0087 & $-0,0016$ & 0,0192 & 0,0569 & 0,0029 & $-0,0005$ & \\
\hline TRIGM & 0,0016 & $-0,0521$ & 0,0143 & 0,0029 & 0,0746 & 0,0030 & \\
\hline GLICM & 0,0036 & $-0,0005$ & $-0,0007$ & $-0,0005$ & 0,0030 & 0,0691 & \\
\hline
\end{tabular}

Tabela D.31: Matriz de covariância estimada dos estimadores dos parâmetros do Modelo de Regressão em Cristas com o quarto parâmetro em cristas. 


\begin{tabular}{|c|c|c|c|c|c|c|c|}
\hline & (Intercepto) & DIAB & TABAG & ANGEST & ANGINS & ARRIT & GENERO \\
\hline (Intercepto) & 0,6930 & $-0,1024$ & $-0,0155$ & $-0,0119$ & $-0,0184$ & $-0,0189$ & $-0,8511$ \\
\hline DIAB & $-0,1024$ & 0,3942 & 0,0007 & 0,0005 & 0,0072 & 0,0123 & 0,0260 \\
\hline TABAG & $-0,0155$ & 0,0007 & 0,0325 & $-0,0004$ & $-0,0015$ & 0,0035 & $-0,0045$ \\
\hline ANGEST & $-0,0119$ & 0,0005 & $-0,0004$ & 0,0346 & 0,0155 & 0,0054 & $-0,0134$ \\
\hline ANGINS & $-0,0184$ & 0,0072 & $-0,0015$ & 0,0155 & 0,0418 & 0,0030 & $-0,0026$ \\
\hline ARRIT & $-0,0189$ & 0,0123 & 0,0035 & 0,0054 & 0,0030 & 0,0770 & 0,0033 \\
\hline GENERO & $-0,8511$ & 0,0260 & $-0,0045$ & $-0,0134$ & $-0,0026$ & 0,0033 & 1,1346 \\
\hline INFARTO & $-0,0145$ & $-0,0103$ & $-0,0053$ & 0,0139 & 0,0121 & 0,0001 & 0,0026 \\
\hline COLM & 0,0169 & 0,0047 & 0,0016 & $-0,0010$ & 0,0007 & 0,0017 & $-0,0275$ \\
\hline IDADFEM & $-0,2829$ & 0,0085 & 0,0046 & $-0,0033$ & 0,0037 & $-0,0063$ & 0,3724 \\
\hline IDADMAS & 0,0901 & $-0,0052$ & 0,0009 & 0,0044 & 0,0064 & $-0,0087$ & $-0,1203$ \\
\hline TRIGM & $-0,0272$ & $-0,0135$ & $-0,0024$ & 0,0006 & $-0,0004$ & $-0,0052$ & 0,0480 \\
\hline \multirow[t]{2}{*}{ GLICM } & 0,0404 & $-0,1623$ & 0,0005 & 0,0015 & $-0,0023$ & $-0,0042$ & $-0,0082$ \\
\hline & INFARTO & COLM & IDADFEM & IDADMAS & TRIGM & GLICM & \\
\hline (Intercepto) & $-0,0145$ & 0,0169 & $-0,2829$ & 0,0901 & $-0,0272$ & 0,0404 & \\
\hline DIAB & $-0,0103$ & 0,0047 & 0,0085 & $-0,0052$ & $-0,0135$ & $-0,1623$ & \\
\hline TABAG & $-0,0053$ & 0,0016 & 0,0046 & 0,0009 & $-0,0024$ & 0,0005 & \\
\hline ANGEST & 0,0139 & $-0,0010$ & $-0,0033$ & 0,0044 & 0,0006 & 0,0015 & \\
\hline ANGINS & 0,0121 & 0,0007 & 0,0037 & 0,0064 & $-0,0004$ & $-0,0023$ & \\
\hline ARRIT & 0,0001 & 0,0017 & $-0,0063$ & $-0,0087$ & $-0,0052$ & $-0,0042$ & \\
\hline GENERO & 0,0026 & $-0,0275$ & 0,3724 & $-0,1203$ & 0,0480 & $-0,0082$ & \\
\hline INFARTO & 0,0605 & 0,0001 & 0,0073 & 0,0088 & 0,0018 & 0,0041 & \\
\hline COLM & 0,0001 & 0,0485 & $-0,0122$ & $-0,0007$ & $-0,0546$ & $-0,0006$ & \\
\hline IDADFEM & 0,0073 & $-0,0122$ & 0,1686 & 0,0050 & 0,0210 & $-0,0040$ & \\
\hline IDADMAS & 0,0088 & $-0,0007$ & 0,0050 & 0,0629 & 0,0014 & 0,0006 & \\
\hline TRIGM & 0,0018 & $-0,0546$ & 0,0210 & 0,0014 & 0,0780 & 0,0034 & \\
\hline GLICM & 0,0041 & $-0,0006$ & $-0,0040$ & 0,0006 & 0,0034 & 0,0782 & \\
\hline
\end{tabular}

Tabela D.32: Matriz de covariância estimada dos estimadores dos parâmetros do Modelo de Regressão em Cristas com o quinto parâmetro em cristas. 


\begin{tabular}{|c|c|c|c|c|c|c|c|}
\hline & (Intercepto) & DIAB & TABAG & ANGEST & ANGINS & ARRIT & GENERO \\
\hline (Intercepto) & 0,0153 & 0,0036 & $-0,0112$ & $-0,0141$ & $-0,0115$ & $-0,0090$ & 0,0098 \\
\hline DIAB & 0,0036 & 0,0025 & $-0,0024$ & $-0,0024$ & $-0,0027$ & $-0,0046$ & 0,0020 \\
\hline TABAG & $-0,0112$ & $-0,0024$ & 0,0325 & $-0,0004$ & $-0,0016$ & 0,0033 & $-0,0093$ \\
\hline ANGEST & $-0,0141$ & $-0,0024$ & $-0,0004$ & 0,0347 & 0,0155 & 0,0053 & $-0,0098$ \\
\hline ANGINS & $-0,0115$ & $-0,0027$ & $-0,0016$ & 0,0155 & 0,0416 & 0,0026 & $-0,0089$ \\
\hline ARRIT & $-0,0090$ & $-0,0046$ & 0,0033 & 0,0053 & 0,0026 & 0,0765 & $-0,0048$ \\
\hline GENERO & 0,0098 & 0,0020 & $-0,0093$ & $-0,0098$ & $-0,0089$ & $-0,0048$ & 0,0088 \\
\hline INFARTO & $-0,0089$ & $-0,0007$ & $-0,0053$ & 0,0141 & 0,0124 & 0,0004 & $-0,0077$ \\
\hline COLM & $-0,0034$ & $-0,0033$ & 0,0017 & $-0,0012$ & 0,0006 & 0,0016 & 0,0015 \\
\hline IDADFEM & 0,0058 & 0,0017 & 0,0030 & $-0,0021$ & 0,0017 & $-0,0090$ & $-0,0056$ \\
\hline IDADMAS & 0,0029 & 0,0013 & 0,0014 & 0,0041 & 0,0072 & $-0,0078$ & $-0,0075$ \\
\hline TRIGM & 0,0060 & 0,0047 & $-0,0025$ & 0,0010 & $-0,0002$ & $-0,0047$ & $-0,0010$ \\
\hline \multirow[t]{2}{*}{ GLICM } & $-0,0022$ & 0,0027 & 0,0018 & 0,0027 & 0,0019 & 0,0029 & $-0,0009$ \\
\hline & INFARTO & COLM & $\overline{\text { IDADFEM }}$ & IDADMAS & TRIGM & GLICM & \\
\hline (Intercepto) & $-0,0089$ & $-0,0034$ & 0,0058 & 0,0029 & 0,0060 & $-0,0022$ & \\
\hline DIAB & $-0,0007$ & $-0,0033$ & 0,0017 & 0,0013 & 0,0047 & 0,0027 & \\
\hline TABAG & $-0,0053$ & 0,0017 & 0,0030 & 0,0014 & $-0,0025$ & 0,0018 & \\
\hline ANGEST & 0,0141 & $-0,0012$ & $-0,0021$ & 0,0041 & 0,0010 & 0,0027 & \\
\hline ANGINS & 0,0124 & 0,0006 & 0,0017 & 0,0072 & $-0,0002$ & 0,0019 & \\
\hline ARRIT & 0,0004 & 0,0016 & $-0,0090$ & $-0,0078$ & $-0,0047$ & 0,0029 & \\
\hline GENERO & $-0,0077$ & 0,0015 & $-0,0056$ & $-0,0075$ & $-0,0010$ & $-0,0009$ & \\
\hline INFARTO & 0,0603 & 0,0006 & 0,0039 & 0,0098 & 0,0009 & 0,0000 & \\
\hline COLM & 0,0006 & 0,0477 & $-0,0025$ & $-0,0035$ & $-0,0531$ & 0,0028 & \\
\hline IDADFEM & 0,0039 & $-0,0025$ & 0,0419 & 0,0430 & 0,0045 & $-0,0021$ & \\
\hline IDADMAS & 0,0098 & $-0,0035$ & 0,0430 & 0,0517 & 0,0062 & $-0,0018$ & \\
\hline TRIGM & 0,0009 & $-0,0531$ & 0,0045 & 0,0062 & 0,0752 & $-0,0044$ & \\
\hline GLICM & 0,0000 & 0,0028 & $-0,0021$ & $-0,0018$ & $-0,0044$ & 0,0087 & \\
\hline
\end{tabular}

Tabela D.33: Matriz de covariância estimada dos estimadores dos parâmetros do Modelo de Regressão em Componentes Principais. 


\section{Referências Bibliográficas}

Agresti(1996) Alan Agresti. An Introduction to Categorical Data Analysis. Wiley Series in Probability and Statistics, primeira edição. Citado na pág. 1

Alkhamisi et al.(2006) M. A. Alkhamisi, G. Khalaf e G. Shukur. Some modification for choosing ridge parameter. Communications in Statistics - Theory and Methods, 35:1-16. Citado na pág. 36

Belsley(1991) David A. Belsley. Conditioning Diagnostics: Collinearity and Weak Data in Regression. Wiley Series in Probability and Statistics, primeira edição. Citado na pág. 17, 25, 26

Belsley et al.(2004) David A. Belsley, Edwin Kuh e Roy E. Welsch. Regression Diagnostics: Identifying Influential Data and Sources of Collinearity. Wiley Series in Probability and Statistics. Citado na pág. 25, 27

Bolfarine e Sandoval(2001) Heleno Bolfarine e Monica C. Sandoval. Introdução à Inferência Estatistica. Sociedade Brasileira de Matemática, primeira edição. Citado na pág. 29

Eubank e Webstern(1985) R. L. Eubank e J. T. Webstern. The singular-value decomposition as a tool for solving estimability problems. The American Statistician, 39:64-66. Citado na pág. 16

Gunst e Mason(1980) Richard F. Gunst e Robert L. Mason. Regression Analysis and its Application. Marcel Dekker, primeira edição. Citado na pág. 20, 23, 24, 36, 39

Hilbe(2009) Joseph M. Hilbe. Logistic Regression Models. Chapman \& Hall, primeira edição. Citado na pág. $1,8,12$

Hill et al.(1977) R. Carter Hill, Thomas B. Fomby e S. R. Johnson. Component selection norms for principal component regression. Communications in Statistics - Theory and Methods, A6:309-334. Citado na pág. 40

Hoerl(1962) Arthur E. Hoerl. Application of ridge analysis to regression problems. Chemical Engineering Progress, 58:54-59. Citado na pág. 29

Hoerl e Kennard(1970) Arthur E. Hoerl e Robert W. Kennard. Ridge regression: Biased estimation for nonorthogonal problems. Technometrics, 42:80-86. Citado na pág. ix, 20, 29, $30,31,32,35$

Hosmer e Lemeshow(1980) David W. Hosmer e Stanley Lemeshow. A goodness of fit test for the multiple logistic regression model. Communications in Statistic, A10:1043-1069. Citado na pág. 11, 12, 13 
Hosmer e Lemeshow(2000) David W. Hosmer e Stanley Lemeshow. Applied Logistic Regression. Wiley Series in Probability and Statistics, segunda edição. Citado na pág. 1, 5, $9,11,75$

Kibria(2003) B. M. G. Kibria. Performance of some new ridge regression estimators. Communications in Statistics - Theory and Methods, 32:419-435. Citado na pág. 36

Lemeshow e Hosmer(1982) Stanley Lemeshow e David W. Hosmer. The use of goodness of fit statisctics in the development of logistic regression models. American Journal of Epidemiology, 115:92-106. Citado na pág. 11

Lesaffre e Marx(1993) Emmanuel Lesaffre e Brian D. Marx. Collinearity in generalized linear regression. Communications in Statistics - Theory and Methods, 22:1933-1952. Citado na pág. 27

Mackinnon e Puterman(1989) Murray J. Mackinnon e Martin L. Puterman. Collinearity in generalized linear models. Communications in Statistics - Theory and Methods, 18: 3463-3472. Citado na pág. 74

Mandel(1982) John Mandel. Use of the singular value decomposition in regression analysis. The American Statistician, 36:15-24. Citado na pág. 16

Mansson e Shukur(2011) Kristofer Mansson e Ghazi Shukur. On ridge parameters in logistic regression. Communications in Statistics - Theory and Methods, 40:3366-3381. Citado na pág. 34,36

Marx e Smith(1990) Brian D. Marx e Eric P. Smith. Principal component estimation for generalized linear regression. Biometrika, 77:23-31. Citado na pág. 37, 39, 40, 74

Montgomery et al.(2001) Douglas C. Montgomery, Elizabeth A. Peck e G. Geoffrey Vining. Introduction to Linear Regression Analysis. Wiley Series in Probability and Statistics, terceira edição. Citado na pág. 24, 25, 26

Neter et al.(2005) John Neter, Michael Kutner, William Wasserman e Christopher Nachtsheim. Applied Linear Statistical Models. McGraw-Hill Higher Education. Citado na pág. 22

Paula(1999) Gilberto A. Paula. Modelos de Regressão com Apoio Computacional. Instituto de Matemática e Estatística (IME) - USP, primeira edição. Citado na pág. 1, 10

Schaefer(1979) Robert L. Schaefer. Multicollinearity and Logistic Regression. Tese de Doutorado, University of Michigan, Ann Harbor, Michigan. Citado na pág. 23

Schaefer(1986) Robert L. Schaefer. Alternative estimators in logistic regression when the data are collinear. Journal of Statistical Computation and Simulation, 25:75-91. Citado na pág. $15,21,37,38,43,44,73$

Schaefer et al.(1984) Robert L. Schaefer, Larry D. Roi e Robert A. Wolfe. A ridge logistic estimator. Communications in Statistics - Theory and Method, 13(1):99-113. Citado na pág. $23,33,34,36$

Seber e Lee(2003) George A. F. Seber e Alan J. Lee. Linear Regression Analysis. Wiley Series in Probability and Statistics, segunda edição. Citado na pág. 18 
Segerstedt(1992) Bo Segerstedt. On ordinary ridge regression in generalized linear models. Communications in Statistics - Theory and Methods, 21:2227-2246. Citado na pág. 74

Singer(2012) Julio M. Singer. Home page. http://www.ime.usp.br/ jmsinger/Dados/ Singer\&Ikeda1996.xls, 2012. Última modificação em 23/05/2012. Citado na pág. 57

Singer e Ikeda(1996) Julio M. Singer e Karina Ikeda. Relatório de análise estatística sobre o projeto: Fatores de risco na doença aterosclerótica coronariana. Relatório Técnico RAE-CEA-9608, Instituto de Matemática e Estatística, Universidade de São Paulo, São Paulo. Citado na pág. 57

Stein(1960) C. M. Stein. Multiple Regression. Contributions to Probability and Statistics. Stanford University Press, primeira edição. Citado na pág. 73

Walker e Duncan(1967) Strother H. Walker e David B. Duncan. Estimation of the probability of an event as a function of several independent variables. Biometrika, 54: 167-179. Citado na pág. 33

Wichern e Churchill(1978) Dean W. Wichern e Gilbert A. Churchill. A comparison of ridge estimators. Technometrics, 20:301-311. Citado na pág. 35 


\section{Índice Remissivo}

Índice e número condição, 25

Aplicação da Regressão em Componentes Principais, 69

Aplicação da Regressão em Cristas, 65

Aplicação dos estimadores viesados, 65

Avaliação do ajuste do modelo, 10

Coeficiente de determinação, 23

Considerações finais do capítulo, 14, 27, 40, 55,70

Decomposição da variância, 26

Decomposição em valores singulares da matriz de planejamento, 16

Delineamento do estudo de simulação, 44

Descrição do estudo, 57

Descrição dos cenários e modelo, 43

Diagnóstico da multicolinearidade na regressão logística, 22

Efeitos da multicolinearidade, 15

Efeitos da multicolinearidade na estimação dos parâmetros, 18

Efeitos da multicolinearidade na inferência sobre os parâmetros, 21

Escolha do parâmetro em cristas, 34

Estimação dos parâmetros, 7

Estimador em Componentes Principais onestep, 38

Estimador em Componentes Principais iterativo, 36

Exclusão dos componentes principais, 40

Identificação da multicolinearidade, 60

Modelo de regressão logística, 3

Regressão em Componentes Principais, 36

Regressão Linear em Cristas, 29

Regressão Logística em Cristas, 33

Relação entre a multicolinearidade em modelos de regressão linear e logística, 19
Resultados, 47

ridge, 29

Teste de significância dos parâmetros, 9 